\title{
Black Hole Entropy \\ in \\ String Theory
}

Huibert het Lam 
$\mathrm{PhD}$ thesis, Utrecht University, May 2020 ISBN: 978-90-393-7306-4

About the cover: this is the first ever direct image of a black hole. The picture was taken of the supermassive black hole at the center of the Messier 87 galaxy. A background of stars has been added in the corners of the original picture.

Image black hole credit: EHT Collaboration.

Cover design by Melodie Zöllner. 


\section{Black Hole Entropy \\ in}

String Theory

\section{Entropie van Zwarte Gaten in Snaartheorie}

(met een samenvatting in het Nederlands)

\section{Proefschrift}

ter verkrijging van de graad van doctor aan de Universiteit Utrecht op gezag van de rector magnificus, prof. dr. H.R.B.M. Kummeling, ingevolge het besluit van het college voor promoties in het openbaar te verdedigen op woensdag 2 september 2020 des middags te 12:45 uur

door

\section{Herbert het Lam}

geboren op 23 december 1991

te Tienhoven aan de Lek 
Promotor: Prof. dr. S.J.G. Vandoren 
To my father,

I wish he could have read this thesis 



\section{Publications}

This thesis is based on the following publications:

1. BPS solutions of six-dimensional $(1,0)$ supergravity coupled to tensor multiplets,

H. het Lam and S. Vandoren, JHEP 06 (2018) 021, arXiv:1804.04681.

2 Four-dimensional black hole entropy from F-theory, T.W. Grimm, H. het Lam, K. Mayer and S. Vandoren, JHEP 01 (2019) 037, arXiv:1808.05228.

3 Black holes and $(0,4)$ SCFTs from type IIB on K3, C. Couzens, H. het Lam, K. Mayer and S. Vandoren, JHEP 08 (2019) 043, arXiv:1904.05361.

4. Twisted $\mathcal{N}=1$ SCFTs and their AdS 3 duals, C. Couzens, H. het Lam and K. Mayer, JHEP 03 (2020) 032, arXiv:1912.07605.

5 Anomalies of $(\mathbf{0 , 4 )}$ SCFTs from F-theory, C. Couzens, H. het Lam, K. Mayer and S. Vandoren, In preparation.

Other publications to which the author contributed (not included in this thesis):

6. Singularities in FLRW spacetimes, H. het Lam and T. Prokopec, Phys. Lett. B775 (2017) 311-314, arXiv:1606.01147.

7. Singularities and conjugate points in FLRW spacetimes, H. het Lam and T. Prokopec,

Gen. Rel. Grav. 49 (2017), no 10, 133, arXiv:1704.03854

8. On the exact entropy of $\mathcal{N}=2$ black holes,

J. Gomes, H. het Lam and G. Mathys, arXiv:1912.00029. 
The papers 2], [3] and 5] also appear in the thesis of K. Mayer (although presented in a different order). We would like to comment on the separate contributions of the author of this thesis and K. Mayer to these papers:

[2] K. Mayer joined this project after the computations in sections 2 and (the two-derivative) part of section 3 in the paper were completed. I performed the computations in sections 2 and 3 of the paper while the computations in section 4 were performed jointly by me and K. Mayer.

[3] I performed most of the computations in section 3 of the paper while $\mathrm{K}$. Mayer performed most of the computations in section 4 .

[5] Since this paper is still in preparation we refer to the relevant sections in this thesis. I performed most of the computations in sections 4.3 and 4.4 while K. Mayer performed most of the computations in section 4.5. 


\section{Contents}

1 Introduction 1

1.1 Motivation . . . . . . . . . . . . . . . . . 1

1.2 Strings to describe the fundamental degrees of freedom . . . . . . 7

1.3 Black holes in string theory $\ldots \ldots \ldots \ldots \ldots \ldots$

1.4 The AdS/CFT correspondence . . . . . . . . . . . . . . . . 39

1.5 Outline $\ldots \ldots \ldots \ldots \ldots \ldots \ldots \ldots$

2 BPS solutions of 6D $(1,0)$ supergravity coupled to tensor multiplets 51

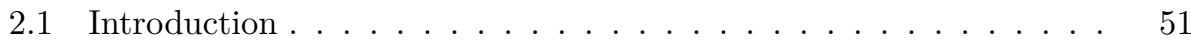

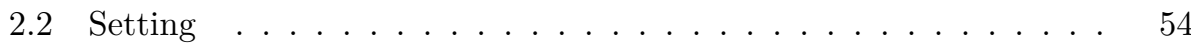

2.3 Supersymmetric solutions $\ldots \ldots \ldots \ldots \ldots \ldots \ldots$

2.4 Classes of solutions $\ldots \ldots \ldots \ldots \ldots$

2.5 Concluding remarks $\ldots \ldots \ldots \ldots \ldots$

2. A Integrability conditions . . . . . . . . . . . . . . . . 79

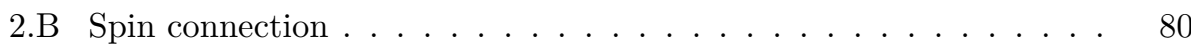

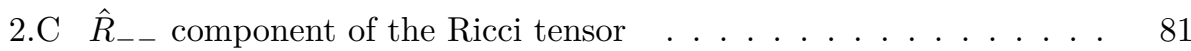

$2 . \mathrm{D}$ Single string solution $\ldots \ldots \ldots \ldots \ldots \ldots$. . . . . . . . 82

3 Black strings in F-theory with Taub-NUT space transversely 85

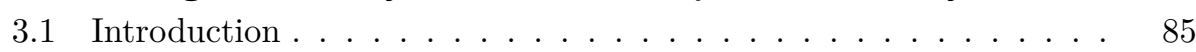

8.2 Microscopics $\ldots \ldots \ldots \ldots$

3.3 Macroscopic classical contributions . . . . . . . . . . . . . . . . 91

3.4 Macroscopic quantum contributions . . . . . . . . . . . . . . . 104

3.5 Summary and $4 \mathrm{D} / 5 \mathrm{D}$ correspondence $\ldots \ldots \ldots \ldots \ldots$

3.6 Discussion . . . . . . . . . . . . . . . . . . . . . . . 113

3.A $6 \mathrm{D}$ to $3 \mathrm{D}$ reduction higher derivative term . . . . . . . . . 114

3.B Action of $\mathbb{Z}_{m}$ and $\mathbb{D}_{m}^{*}$ on KK spectrum . . . . . . . . . . . 116

3.C $6 \mathrm{D}$ to 5D one-loop corrections $\ldots \ldots \ldots \ldots \ldots \ldots$

3.D Summation of $6 \mathrm{D}$ to 3D one-loop corrections . . . . . . . . . . . 119

$\begin{array}{|lll|}4 & \text { Black strings in F-theory with ALE/ALF spaces transversely } & 129\end{array}$

4.1 Introduction . . . . . . . . . . . . . . . . . . . . . . . . . . 129 
4.2 ALE/ALF transverse spaces . . . . . . . . . . . . . . . . . . 131

4.3 Macroscopic classical contributions . . . . . . . . . . . . . . . . . . 133

4.4 Macroscopic quantum contributions for ALF transverse spaces . . 139

4.5 Non-abelian flavour symmetries . . . . . . . . . . . . . . . . . . 142

4.6 Summary and discussion . . . . . . . . . . . . . . . . . . . . . . . . 149

$5 \quad$ Black strings in type IIB with ALE/ALF spaces transversely 153

5.1 Introduction. . . . . . . . . . . . . . . . 153

5.2 Macroscopics . . . . . . . . . . . . . . . . 155

5.3 Microscopics . . . . . . . . . . . . . . . . . . . 163

$5 . \mathrm{A}$ Summation of $6 \mathrm{D}$ to 3D one-loop corrections . . . . . . . . . . . 170

5.B $U(1)_{L}$ charges of $\mathcal{N}=2$ fields $\ldots \ldots \ldots \ldots$. . . . . . . . 177

6 Twisted $\mathcal{N}=1$ SCFTs and their $\mathrm{AdS}_{3}$ duals 179

6.1 Introduction . . . . . . . . . . . . . . . 179

6.2 Twisted $\mathcal{N}=1$ field theories and c-extremization . . . . . . . . . . 183

6.3 Geometric dual of c-extremization . . . . . . . . . . . . . . . 190

6.4 Explicit supergravity solutions . . . . . . . . . . . . . . . . . 200

6.5 Discussion . . . . . . . . . . . . . . . . . . . . . 222

6. A Ansatz analysis details . . . . . . . . . . . . . . . . . 223

6.B Effective coordinate systems . . . . . . . . . . . . . . . . 224

\begin{tabular}{ll}
\hline Summary & 227
\end{tabular}

\begin{tabular}{ll}
\hline Samenvatting & 231
\end{tabular}

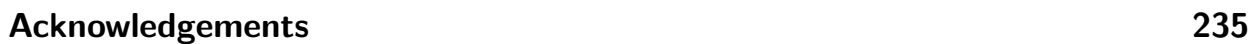

\begin{tabular}{|ll}
\hline About the author & 237
\end{tabular}

\begin{tabular}{ll}
\hline Bibliography & 239
\end{tabular} 


\section{Introduction}

The purpose of this chapter is to familiarize the reader with the central topic of this thesis. Namely, how can we understand the entropy of black holes from a counting of microstates? For this, a theory of quantum gravity is needed and the particular candidate we work with is string theory. We will introduce this theory and explain how microstates of black holes are counted. We will also give an overview of the work presented in this thesis.

\subsection{Motivation}

Black holes are some of the most fascinating objects in physics. Simply said, these are objects that are so dense that nothing, not even light, can escape their surface. They were first considered in the 18th century, long before the era of modern physics which began in the 20th century with the breakthroughs of relativity and quantum mechanics. Assuming light consists of particles, John Michell considered objects with so much mass that their escape velocity, the velocity needed to escape the gravitational pull of the object, exceeds the speed of light. These objects were called dark stars by John Michell because of the inability to see them directly; the term black hole originated only in the 1960s. Interest in these objects vanished, like light into a black hole, after Thomas Young's 1801 double-slit experiment demonstrated the wavelike nature of light and the particle view of light got displaced by the wave view. However in 1916, soon after the development of general relativity, a solution was published describing a spherically symmetric black hole. Named after its founder, the Schwarzschild black hole is described by only its mass $M{ }^{1}$

$$
\mathrm{d} s^{2}=-\left(1-\frac{2 G M}{r}\right) \mathrm{d} t^{2}+\left(1-\frac{2 G M}{r}\right)^{-1} \mathrm{~d} r^{2}+r^{2}\left(\mathrm{~d} \theta^{2}+\sin ^{2}(\theta) \mathrm{d} \phi^{2}\right),
$$

where we have used spherical coordinates and $G$ is the gravitational constant. This solution is characterized by a singularity at $r=0$ and a coordinate singularity at the event horizon $R_{\text {sch }}=2 G M$ which is an artifact of the coordinate system used and disappears after a change of coordinates. Classically, nothing, not even

\footnotetext{
${ }^{1}$ We use units in which $\hbar=k_{\mathrm{B}}=c=1$.
} 
light, inside the event horizon can reach an observer outside of the black hole. The solution 1.1 can be generalized to be charged and spinning. Of course due to the non-linear nature of the Einstein equations closed form solutions cannot always be written down. With the theorems of Penrose and Hawking [9 11] the singularity was also proven to be a characteristic of more general black hole solutions in general relativity, i.e. they showed that under a few general assumptions there always is a singularity once an event horizon has been formed. For a long time however, black hole solutions were seen as theoretical curiosities which are not realized in nature. This changed drastically with the discovery of neutron stars in 1967 . These neutron stars are expected to form when massive stars collapse at the end of their life cycle. Neutron stars consist of neutrons which are fermions and thus subject to the Pauli exclusion principle. Under high densities they form a degenerate gas counteracting the gravitational force by degeneracy pressure. When a star is very massive, neutron pressure is not enough for stability and one expects a stellar-mass black hole to form at the end of its lifetime. Therefore the existence of neutron stars suggests their heavier cousins could also exist. Despite light being unable to escape a black hole, their presence may be detected by their interaction with other matter and light. For instance, infalling matter can form an accretion disk heated by friction such that it can be observed. Also, by studying the movement of stars orbiting the black hole, one may infer its mass and location. By using such techniques it has been established that Sagittarius $A^{*}$, a radio source in the core of the Milky Way, contains a supermassive black hole of about 4.3 million solar masses. It is also believed that other galaxies have supermassive black holes in their centers. Another object that is widely accepted to be a black hole is the X-ray source Cygnus X-1. It has a mass of about 14.8 solar masses and is part of a binary system, accreting matter from its companion star. Also other candidate black holes have been found in X-ray binaries. In the last couple of years the experimental evidence for the existence of black holes has increased. For the first time in 2015 the merger of two black holes has been observed by the LIGO and Virgo collaboration via the detection of gravitational waves $[12$. Since this first detection multiple other mergers have been observed. In 2019 the first ever direct image of a black hole was published by the Event Horizon Telescope 13 . This picture was taken of the supermassive black hole at the center of the Messier 87 galaxy. Thus there is ample evidence that black holes are objects in our universe.

$\overline{{ }^{2} \text { This image can be found on the cover }}$ of this thesis. 


\subsubsection{Black holes as thermodynamic systems}

In the early seventies, Hawking, Bardeen, Bekenstein and Carter showed that black holes behave as thermodynamic systems [14 16, i.e. black holes obey certain laws that mimic the laws of thermodynamics. Imagine for instance matter falling into the Schwarzschild black hole (1.1). This process increases the black hole's mass which means that the area of the event horizon $3=8 \pi^{2} G M$ increases. Since nothing can escape from within the horizon it means that the surface area cannot decrease. In fact it can be shown that under certain reasonable assumptions every physical process results in the increase of the surface area of the event horizon. This never decreasing quantity is reminiscent of the second law of thermodynamics. In order to state the full set of laws we must first introduce the surface gravity $\kappa$, associated to the event horizon of a black hole. Suppose the horizon is a Killing horizon, i.e. it is a null-hypersurface $\Sigma$ for which there exists a Killing vector field $\xi$ that is normal to $\Sigma$. Then the surface gravity $\kappa$ is defined as

$$
\kappa^{2}=-\left.\frac{1}{2}\left(\nabla^{\mu} \xi^{\nu}\right)\left(\nabla_{\mu} \xi_{\nu}\right)\right|_{\Sigma}
$$

where $\mu=0, \ldots, 3$. For stationary axisymmetric and static black holes it can be shown that their event horizon is a Killing horizon [11, 17. It is clear from 1.2 that the definition of $\kappa$ depends on the normalization of $\xi$. The black holes we consider always have their horizon for a specific value of a radial coordinate $r$. Motivated by the fact that in most of this thesis we are interested in asymptotically flat black hole solutions, we require that the normalization of $\xi$ for large values of $r$ equals the one of $\partial_{t}$ in Minkowski space. We thus fix $\xi$ such that

$$
\lim _{r \rightarrow \infty} \xi^{2}=-1
$$

The laws of black hole mechanics are then formulated as follows:

Oth law. The surface gravity $\kappa$ is constant along the event horizon.

1st law.

$$
\mathrm{d} M=\frac{1}{8 \pi G} \kappa(M, Q, J) \mathrm{d} A+\Omega(M, Q, J) \mathrm{d} J+\mu(M, Q, J) \mathrm{d} Q,
$$

where $M, Q$ and $J$ are the mass, charge and angular momentum of the black hole.

\footnotetext{
${ }^{3}$ When we talk about the area of the horizon we actually mean the area of a spacelike twodimensional cross section of the event horizon. Technically it is defined to be the surface on which the Killing vector $\xi$ introduced below vanishes.
} 
2nd law. The area $A$ of the event horizon does not decrease in time:

$$
\mathrm{d} A \geq 0
$$

3rd law. It is impossible, by any process to reduce the surface gravity $\kappa$ to zero in a finite number of operations. It is possible to rephrase the last condition in a more formal way, but this is outside the scope of this thesis.

In table 1.1 we compare these laws with the normal laws of thermodynamics. From this we identify the temperature and entropy to be

$$
T=\frac{1}{8 \pi \alpha} \kappa, \quad S=\frac{\alpha}{G} A,
$$

where $\alpha$ is a constant. Note that at this stage, this is purely a formal identification since a temperature is associated to an equilibrium between a system and its surrounding and classically a black hole does not radiate anything. Furthermore, for thermodynamic systems an entropy is associated to the number of microstates $d$ via

$$
S=\log d
$$

One would therefore like to continue the analogy by speculating that the entropy of a black hole counts the number of microstates of the black hole. However, the no-hair conjecture [18] seems to rule out such an identification. Inspired by the uniqueness theorems 11, 19,22 , the no-hair conjecture states that a stationary, asymptotically flat black hole in four dimensions is fully characterized by its mass, charge and angular momentum. So what do these microstates correspond to?

\begin{tabular}{ccc}
\hline Law & Thermodynamics & Black holes \\
\hline 0th & $\begin{array}{c}\text { In thermal equilibrium } T \text { is } \\
\text { constant everywhere }\end{array}$ & $\begin{array}{c}\text { For stationary black holes } \kappa \text { is } \\
\text { constant on the event horizon }\end{array}$ \\
\hline 1st & $\mathrm{d} E=T \mathrm{~d} S+\Omega \mathrm{d} J+\mu \mathrm{d} Q$ & $\mathrm{~d} M=\frac{\kappa}{8 \pi G} \mathrm{~d} A+\Omega \mathrm{d} J+\mu \mathrm{d} Q$ \\
\hline 2nd & $\mathrm{d} S \geq 0$ & $\mathrm{~d} A \geq 0$ \\
\hline 3rd & $\begin{array}{c}T=0 \text { cannot be achieved in } \\
\text { a finite number of operations }\end{array}$ & $\begin{array}{c}\kappa=0 \text { cannot be achieved in } \\
\text { a finite number of operations }\end{array}$ \\
\hline
\end{tabular}

Table 1.1: Comparison of the laws of thermodynamics and the laws of black hole mechanics. 
Not long after the laws of black holes mechanics were formulated, Hawking showed that semi-classically black holes actually do radiate 23. The emitted Hawking radiation has a thermal spectrum and the associated temperature is given by

$$
T=\frac{\kappa}{2 \pi}
$$

which fixes the coefficient $\alpha$ in 1.6 . In particular this implies that the entropy of a black hole is given by the Bekenstein-Hawking area law

$$
S=\frac{A}{4 G} \text {. }
$$

The laws of black hole mechanics are only valid for black holes that have $\kappa \neq 0$, however we can define the entropy of a black hole with $\kappa=0$ as a limit of $(1.9)$ of black holes with $\kappa \neq 0$. This will be important because all black holes we will encounter in this thesis are extremal, i.e. have vanishing surface gravity.

The Bekenstein-Hawking entropy formula 1.9 is only valid for two-derivative gravity, however as we will see in section 1.2 .2 the actions we are interested in may have higher derivative terms. For such more generic actions the correct expression for the entropy is given by the Wald entropy formula 24,25

$$
S=-2 \pi \int_{\text {hor }} \mathrm{d}^{2} \Omega \sqrt{h} \frac{\delta \mathcal{L}}{\delta R_{\mu \nu \rho \lambda}} \epsilon_{\mu \nu} \epsilon_{\rho \lambda},
$$

where the integration is over the spacelike two-dimensional cross section of the horizon and $h$ is the induced metric. The binormal vector $\epsilon_{\mu \nu}$ for the horizon is defined as

$$
\epsilon_{\mu \nu}=\frac{1}{\kappa} \nabla_{\mu} \xi_{\nu}
$$

We can check the formula 1.10 for the Schwarzschild black hole 1.1 which is a solution of two-derivative gravity with Lagrangian density

$$
\mathcal{L}=\frac{1}{16 \pi G} R
$$

The Killing vector can be identified as $\xi=\partial_{t}$. We then find that $\epsilon_{t r}=-\epsilon_{r t}=1$ and

$$
\frac{\delta \mathcal{L}}{\delta R_{\mu \nu \rho \lambda}} \epsilon_{\mu \nu} \epsilon_{\rho \lambda}=\frac{1}{16 \pi G} g^{\mu \rho} g^{\lambda \nu} \epsilon_{\mu \nu} \epsilon_{\rho \lambda}=-\frac{1}{8 \pi G}
$$

The Wald entropy is indeed equal to the Bekenstein-Hawking entropy for this case.

To understand the entropy in terms of microstates one needs a theory of quantum gravity. We know how to quantize quantum field theories (QFTs), but when treating 
general relativity in this framework it turns out to be non-renormalizable. The theory of general relativity should be seen as an effective theory, it is only valid up to a certain energy scale which we call the cutoff of the theory. The phenomenon that a certain description is only valid for a certain energy scale happens everywhere in physics. For instance, to describe the flow of water one can rely on hydrodynamics without knowing the details of the molecules that the water is made of. The cutoff scale of general relativity is not known, but the theory certainly breaks down above the scale set by the Planck mass,

$$
M_{\mathrm{pl}}=\sqrt{\frac{\hbar c}{G}} \approx 10^{19} \mathrm{GeV} / c^{2},
$$

which is the unique quantity with mass dimension that can be constructed out of the fundamental constants $\hbar, c$ and $G$. To describe gravity at all scales we need a theory of quantum gravity. Unfortunately, this theory is not known yet; however, there are a few candidates. We will work with one of these candidates in this thesis called string theory.

\subsubsection{Purpose of this thesis}

A theory of quantum gravity should be able to account for the microstates of black holes. Therefore, one can use black holes as a theoretical testing ground for such theories. It turns out that string theory is indeed able to account for the entropy of certain black holes. The purpose of this thesis is to further the understanding of this microstate counting. On the one hand we do this by studying the entropy of black holes that were not previously studied. On the other hand we compute corrections to the formula 1.9 by computing the full Wald entropy 1.10 both from a macroscopic point of view and from string theory, and showing that both sides match.

Before we can do this we must introduce string theory (including M- and Ftheory), its low-energy limits and its compactifications. This is the subject of section 1.2 Following this introduction, we discuss in section 1.3 how black holes arise in string theory and how their microstates can be counted. In section 1.4 we introduce the so-called AdS/CFT correspondence which provides an alternative way of looking at the microstate counting program. Namely we explain that this counting is a test of the correspondence. Lastly, in section 1.5 we provide an overview of the work that is presented in this thesis. 


\subsection{Strings to describe the fundamental degrees of freedom}

The fundamental idea behind string theory is rather simple: use strings instead of point-particles to describe the fundamental degrees of freedom. There are two kind of strings: closed strings with the topology of a circle that move around in space and open strings that have the topology of a line segment and have their endpoints fixed to a multi-dimensional surface called a $\mathrm{D} p$-brane. See figure 1.1 for an illustration. The strings are characterized by a length scale $\ell_{s}$ which is so small that the effects of spatial extension can only be seen at small scales or equivalently at very high energies $4^{4}$ Particles correspond to oscillation modes of the strings and have masses scaling with $M_{s} \sim \ell_{s}^{-1}$. At energies $E \ll M_{s}$ we can integrate out the massive modes and one effectively only sees the massless particles. Only at energies near the scale set by $\ell_{s}$ do the effects of the extended nature of the strings appear. One of the massless excitation modes of the closed string always leads to a particle with spin two which is interpreted as the graviton mediating the gravitational force when general relativity is treated as a QFT. More importantly, the Einstein equation follows from consistency conditions of string theory. Therefore, a theory

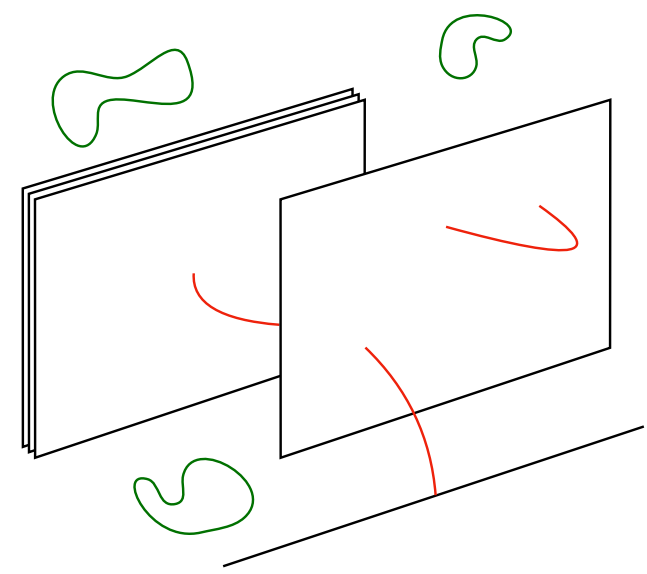

Figure 1.1: String theory contains two kind of strings. In this figure the open strings are red and have their endpoints fixed to multi-dimensional surfaces called D-branes. The closed strings are green and move around in space.

${ }^{4}$ The length scale $\ell_{s}$ is typically taken to be of the order of the Planck length but should be fixed by experiment. 
of strings includes gravity. Consistency of string theory also requires spacetime to be ten-dimensional. The theory can describe our four-dimensional universe when six of these dimensions are compact and so small that they are inaccessible for the current experiments. Furthermore, string theory is likely to be valid at arbitrary high energies. Intuitively the extended nature of strings prohibits being able to probe length scales smaller than $l_{s}$. However, the UV-completeness of string theory, which is supported by strong arguments and therefore widely accepted, has only been proven at two loops.

Apart from the graviton, many other particles appear in string theory. Thus string theory is potentially also a candidate for a theory of everything, a theory that combines gravity with the other three forces that are currently described using the Standard Model. The latter model unifies three of the four observed interactions, namely the electromagnetic force, the weak force and the strong force. It is a QFT or more precisely a gauge theory with gauge group $S U(3) \times S U(2) \times U(1)$ where the interactions are mediated by gauge bosons.

We begin by describing the different string theories in section 1.2.1. Following this we discuss the low energy limit of string theory in section 1.2.2. Subsequently we describe in section 1.2 .3 how a ten-dimensional theory of strings can appear four-dimensional via the concept of compactification. For the construction of black holes we will need to study D-branes in more depth and this is the topic of section 1.2.4 In section 1.2.5 we then end with a discussion of F-theory which is the playground of chapters 3 and 4 . Note that we do not intend to discuss string theory in depth here, a more complete introduction can be found in text books such as 26 30]. We rather focus on the parts important for this thesis.

\subsubsection{String theories and M-theory}

String theory was originally developed in the late 1960s and early 1970s as a theory to describe hadrons. Although that attempt was not successful and quantum chromodynamics was recognized as a better theory of hadrons $5^{5}$ it was discovered that general relativity arises in the low-energy limit of string theory, which is therefore a candidate for a theory of quantum gravity. To describe how exactly this comes about, we first need to take a step back and ask ourselves what a theory of strings looks like.

The most straightforward idea is to generalize the action describing a point-

\footnotetext{
${ }^{5}$ However, string theory might contain quantum chromodynamics such that it would describe hadrons after all.
} 
particle in flat $D$-dimensional space

$$
S=-m \int \mathrm{d} s=-m \int \mathrm{d} \tau \sqrt{-\partial_{\tau} X^{\mu} \partial_{\tau} X^{\nu} \eta_{\mu \nu}},
$$

where $\mu=0, \ldots, D-1$. The action minimizes the worldline of the point-particle and therefore the generalization to strings is to minimize the worldsheet of the string. This generalization is given by the Nambu-Goto action

$$
S_{\mathrm{NG}}=-T_{s} \int \mathrm{d} A=-T_{s} \int \mathrm{d}^{2} \sigma \sqrt{-(\dot{X})^{2}\left(X^{\prime}\right)^{2}+\left(\dot{X} \cdot X^{\prime}\right)^{2}},
$$

and gives the classical action of the bosonic string. Here $\sigma^{\alpha}=(\tau, \sigma), \alpha=1,2$, are the coordinates on the worldsheet of the string, $\dot{X}=\partial_{\tau} X, X^{\prime}=\partial_{\sigma} X$ and

$$
T_{s}=\frac{1}{2 \pi \alpha^{\prime}}=\frac{1}{2 \pi \ell_{s}^{2}}
$$

is the tension of the string. For closed strings we take $\sigma$ to be periodic, i.e. $X^{\mu}(\tau, \sigma)=X^{\mu}(\tau, \sigma+2 \pi)$. Because of the square root the Nambu-Goto action 1.16 is difficult to quantize and therefore it is useful to rewrite it into the classically equivalent form

$$
S_{\mathrm{P}}=-\frac{1}{2} T_{s} \int \mathrm{d}^{2} \sigma \sqrt{-g} g^{\alpha \beta} \partial_{\alpha} X^{\mu} \partial_{\beta} X^{\nu} \eta_{\mu \nu}
$$

This is the Polyakov action and as one can see the square root is eliminated at the expense of an extra field. One can show the classical equivalence to the Nambu-Goto action by substitution of the equation of motion for $g^{\alpha \beta}$. We would like to point out one symmetry of the action 1.18 which will have rather strong implications. This is a gauge symmetry called Weyl symmetry and is given by sending $g_{\alpha \beta}\left(\sigma^{\beta^{\prime}}\right) \rightarrow$ $f\left(\sigma^{\beta^{\prime}}\right) g_{\alpha \beta}\left(\sigma^{\beta^{\prime}}\right)$, where $f$ is a function of the worldsheet coordinates. This symmetry combined with reparametrization invariance of 1.18 can be used to fix $g_{\alpha \beta}=\eta_{\alpha \beta}$, after which the equation of motion for $X^{\mu}$ reduces to the free wave equation. When choosing lightcone coordinates on the worldsheet it then becomes clear that the most general solution to this equation is the sum of a left-moving wave and a right-moving wave. Of course one still has to impose the constraints coming from the equation of motion for $g_{\alpha \beta}$. It is possible to quantize the string. Keeping the Weyl symmetry in the quantized theory, as one should for a gauge symmetry, fixes the dimension of spacetime to $D=26{ }^{6}$ Particles can be identified with excitations of the string where different oscillation modes correspond to different particles.

\footnotetext{
${ }^{6}$ This critical dimension is needed if one wants to quantize string theory in Minkowski space without any additional degrees of freedom. Otherwise one can also have bosonic string theory in dimensions other than 26. We will not discuss these non-critical strings.
} 
There are two issues with the bosonic string. The first one is that the particle spectrum contains a tachyon, i.e. a particle with negative mass-squared. In quantum field theory language this indicates that we are expanding the theory around a maximum of the potential of the tachyon field which is unstable and signifies that the theory will decay into a lower energy state. This does not necessarily imply that the full theory is doomed. For example the Higgs particle of the Standard Model can be viewed as a tachyon when the theory is expanded around the maximum of the Higgs potential, and we do not discard this theory. The problem for bosonic string theory is that there is no understanding of what the theory decayed into or whether this decayed state was even stable. The second problem is more significant: the particle spectrum does not contain fermions. Since all matter in our universe is made out of fermions, we definitely need them. These problems can be solved by adapting the Polyakov action 1.18 by introducing supersymmetry on the worldsheet. This leads to superstring theory. Supersymmetry is a symmetry relating bosons and fermions and a theory with supersymmetry contains as many integer-valued spin as halfinteger-valued spin degrees of freedom. After quantization of the superstring action, the preservation of Weyl symmetry quantum mechanically fixes the dimension of spacetime to be $D=10$. There are a number of discrete choices one can make in constructing the superstring. The most important one is whether one chooses to make only one of the left- and right-moving sectors supersymmetric or both of the sectors. Let us first discuss the possibility that both sectors are supersymmetric. Then one additionally has the choice between open and closed strings, oriented and unoriented strings and the different boundary conditions for the fermionic fields. Unoriented strings are invariant under the transformation $\sigma \rightarrow 2 \pi-\sigma$. We have already seen the boundary conditions for the bosonic closed string, namely we required the fields to be periodic, i.e. $X^{\mu}(\tau, \sigma)=X^{\mu}(\tau, \sigma+2 \pi)$. We must also specify the boundary condition for the left-moving $\psi$ and right-moving $\bar{\psi}$ fermions on the worldsheet. There are two such possibilities:

- $\operatorname{Ramond}(\mathrm{R}): \psi^{\mu}(\tau, \sigma)=\psi^{\mu}(\tau, \sigma+2 \pi)$,

- Neveu-Schwarz (NS): $\psi^{\mu}(\tau, \sigma)=-\psi^{\mu}(\tau, \sigma+2 \pi)$,

where the sign is the same for all $\mu$ as required by spacetime Poincaré invariance. We have similar choices for $\bar{\psi}$. This thus leads to four possibilities R-R, R-NS, NS-NS and NS-R. For the open string there are also choices to be made, we discuss this further in section 1.2.4.1. It turns out that out of all the choices we can make, three consistent string theories can be constructed:

- Type I based on unoriented closed and open strings, 
- Type IIA based on oriented closed strings,

- Type IIB based on oriented closed strings.

First we would like to note that there can be interactions between open strings resulting in a closed string. This is why a theory of open strings necessarily contains closed strings. It is however possible to have a theory with only closed strings and the type II theories are examples. Their spectrum can be defined via a so-called GSO projection on the spectrum one gets after quantization. Two inequivalent projections lead to the A and B versions. Even though open strings are not part of type II in the description so far, they will arise non-perturbatively as we will see in section 1.2.4 In type I the inclusion of open strings is demanded for consistency. The part of the spectrum coming from the closed strings is a projection of the spectrum of type IIB.

As we mentioned there is one other choice that we have not yet considered, namely only turning on supersymmetry in one of the sectors. With this construction two additional theories have been found, namely the heterotic string theories:

- $S O(32)$ heterotic string theory,

- $E_{8} \times E_{8}$ heterotic string theory,

which differ in their gauge group as indicated.

It turns out that apart from supersymmetry on the worldsheet we also have spacetime supersymmetry in all the superstring theories, i.e. the spectrum falls into supersymmetric representations. Every symmetry comes with a conserved charge and the ones corresponding to the supersymmetries are called supercharges. The amount of supersymmetry is thus indicated by the number of supercharges. Supercharges fall in a spinor representation and the number of the spinors is often indicated by a number $\mathcal{N}$. Generally it refers to the irreducible spinor in the dimension under consideration. Since the number of components of this irreducible spinor is different for different dimensions, the same amount of supersymmetry comes with a different number of $\mathcal{N}$ in the different dimensions. For instance $\mathcal{N}=1$ supersymmetry in 10 dimensions corresponds to one Majorana-Weyl spinor which has 16 real components. In four dimensions 16 real supercharges correspond to 2 Majorana spinors and thus $\mathcal{N}=2$. The spectrum of the type II theories has $\mathcal{N}=2$ supersymmetry as already indicated by their name. The theories differ in the chirality of the two Majorana-Weyl spinors, type IIA is non-chiral and type IIB is chiral. The heterotic and type I string theories have $\mathcal{N}=1$ supersymmetry or 16 real supercharges. 
From now on we just focus on the type II string theories since they will be the playground for most of this thesis. We would like to first present the massless part of the bosonic spectrum of these theories. The bosons come from the R-R and NS-NS sectors, the other two sectors give rise to the fermions. Both theories have the same massless particles from the NS-NS sector, namely the dilaton $\Phi$, which is a scalar, the Kalb-Ramond field $B_{2}$, which is a two-form, and the metric $g_{\mu \nu}$. The theories differ in the R-R sector, type IIA has a one-form $C_{1}$ and a three-form $C_{3}$, and type IIB has a zero-form $C_{0}$, also known as the axion, a two-form $C_{2}$ and a four-form $C_{4}$. The forms $B$ and $C_{(i)}$ should be thought of as gauge fields and come with a gauge-invariant field strength $H_{3}=\mathrm{d} B_{2}$ and $F_{i+1}=\mathrm{d} C_{i}$. The vacuum expectation value of the dilaton determines the strength of string interactions given by the string coupling $g_{s}$ via

$$
g_{s}=e^{\langle\Phi\rangle} .
$$

So far we have seen that there are at least five different string theories which thus all are potential theories of quantum gravity. That there are different possibilities is not very satisfying since one would like the fundamental theory of nature to be unique in some sense. Fortunately, all the different string theories are actually dual to each other. For instance type IIA on a circle with radius $R$ is equivalent to type IIB on a circle with radius $\frac{\alpha^{\prime}}{R}$. This symmetry is called T-duality. In a sense the two type II theories are the same. Moreover all the other theories are related to each other via various dualities. We will not go into the details here and refer the reader to one of the many textbooks on the subject. The theories can thus be seen as different descriptions of the same theory. These descriptions are all perturbative, but one can ask what happens in the strong coupling limit. It turns out that for type IIB this limit is related to weak coupling via an $S L(2, \mathbb{Z})$ symmetry. We will study this symmetry better in section 1.2 .2 In the strong coupling limit of type IIA a circle opens up and the theory is described by an eleven-dimensional theory called M-theory. Type IIA thus arises by compactifying the $11 \mathrm{~d}$ theory on the so-called M-theory circle 7 A complete formulation of the 11d theory is not known, but its low-energy limit is given by the unique supergravity theory in eleven dimensions which we will briefly discuss in the subsequent section.

\subsubsection{Low energy actions}

When energies satisfy $E \ll \ell_{s}^{-1}$ we can focus on just the massless part of the spectrum since the massive fields all have masses $\gg E$, see the discussion above.

${ }^{7}$ We explain in more detail how this works in sections 1.2 .3 .1 and 1.2 .4 .2 
One can associate fields in spacetime to these modes such that the string excitations can be identified with a quantum of these fields. The dynamics of these fields is then governed by the action that reproduces the string scattering amplitudes of the massless string modes and obeys the spacetime symmetries of the theory. Since $\alpha^{\prime}$ is the only dimensionful constant, the expansion of the action in number of derivatives will be an expansion in powers of $\sqrt{\alpha^{\prime}}$. Higher derivative corrections become important for high curvatures. We will not go into the details of the derivation of the action but just state the two-derivative result for the type II theories which can be written as

$$
S_{\mathrm{II}}=S^{\mathrm{R}}+S^{\mathrm{NS}}+S^{\mathrm{ferm}}
$$

The last term is the action for the massless fermions. Since the fermions vanish in classical solutions, there is no need to further discuss this part of the action. The part $S^{\mathrm{NS}}$ describes the dynamics of the NS-NS sector which is the same for type IIA and type IIB:

$$
S_{\mathrm{II}}^{\mathrm{NS}}=\frac{1}{2 \kappa_{10}^{2}} \int e^{-2 \Phi}\left[R_{10} * 1-\frac{1}{2} H_{3} \wedge * H_{3}+4 \mathrm{~d} \Phi \wedge * \mathrm{~d} \Phi\right],
$$

where $\kappa_{10}^{2}=\frac{1}{4 \pi}\left(4 \pi^{2} \alpha^{\prime}\right)^{4}{ }^{8}$ The two type II theories differ in their R-R sector with dynamics described by

$$
\begin{aligned}
& S_{\mathrm{IIA}}^{\mathrm{R}}=-\frac{1}{4 \kappa_{10}^{2}} \int\left[F_{2} \wedge * F_{2}+\tilde{F}_{4} \wedge * \tilde{F}_{4}+B_{2} \wedge F_{4} \wedge F_{4}\right], \\
& S_{\mathrm{IIB}}^{\mathrm{R}}=-\frac{1}{4 \kappa_{10}^{2}} \int\left[F_{1} \wedge * F_{1}+\tilde{F}_{3} \wedge * \tilde{F}_{3}+\frac{1}{2} \tilde{F}_{5} \wedge * \tilde{F}_{5}+C_{4} \wedge H_{3} \wedge \tilde{F}_{3}\right] .
\end{aligned}
$$

We have defined

$$
\begin{aligned}
& \tilde{F}_{3}=F_{3}-C_{0} H_{3}, \\
& \tilde{F}_{4}=F_{4}+C_{1} \wedge H_{3}, \\
& \tilde{F}_{5}=F_{5}-\frac{1}{2} C_{2} \wedge H_{3}+\frac{1}{2} B_{2} \wedge F_{3} .
\end{aligned}
$$

The action 1.22 for type IIB gives rise to all equations of motion apart from a self-duality condition on $\tilde{F}_{5}$ :

$$
\tilde{F}_{5}=* \tilde{F}_{5}
$$

${ }^{8}$ The constant is fixed by requiring the tension of the fundamental string to be equal to the tension of the D1-brane that we will encounter in section 1.2 .4 see e.g. 30. 
which cannot be easily implemented in terms of a Lagrangian because it makes the kinetic term for $C_{4}$ vanish. Therefore, 1.22 is also called a pseudo-action and the self-duality condition has to be imposed at the level of the equations of motion. Note that the last term in both actions 1.21 and 1.22 is a Chern-Simons term and thus does not depend on the metric.

The low-energy actions 1.19 for the type II theories are examples of supergravity actions which are supersymmetric extensions of the Einstein-Hilbert action. Since the spectrum of the superstrings is supersymmetric, obtaining a supergravity action in their low-energy limit is not surprising. The actions 1.19 are actually the unique $10 \mathrm{~d}$ two-derivative ones that have $\mathcal{N}=2$ supersymmetry. There is also a supergravity action that has $\mathcal{N}=1$ supersymmetry and this turns out to be the low-energy limit of the type I theory and the heterotic string theories. It has a gauge group which has to be either $S O(32)$ or $E_{8} \times E_{8}$ in order for anomalies to cancel. The type I and $S O(32)$ heterotic string theories have exactly the same low-energy limit. The group $E_{8} \times E_{8}$ is realized as the low-energy limit of the other heterotic string theory.

There is a unique $11 \mathrm{~d}$ supergravity action and this is the low-energy limit of M-theory. Its bosonic field content consists of a metric and a three-form field $A_{3}$ and the action is given by

$$
S_{\mathrm{M}}=\frac{1}{2 \kappa_{11}^{2}} \int\left[R_{11} * 1-\frac{1}{2} \mathrm{~d} A_{3} \wedge * \mathrm{~d} A_{3}-\frac{1}{6} A_{3} \wedge \mathrm{d} A_{3} \wedge \mathrm{d} A_{3}\right] .
$$

Note that we have given the type II actions in the so-called string frame and that the kinetic terms in 1.20 are not canonically normalized. However, with a field redefinition the action can be rewritten in Einstein frame where it only contains terms with the proper normalization. Generally when one has a $D$-dimensional action with the Ricci scalar appearing as in 1.20 , one can transform to Einstein frame using the field redefinition

$$
g_{\mu \nu}^{\mathrm{E}}=e^{-\frac{4}{D-2} \Phi} g_{\mu \nu} .
$$

So for the type II action the transformation is given by $g_{\mu \nu}^{\mathrm{E}}=e^{-\Phi / 2} g_{\mu \nu}$. We now write the type IIB action in Einstein frame in order to show that it is invariant under an $S L(2, \mathbb{R})$ symmetry. The part of the action 1.20 becomes

$$
S_{\mathrm{II}}^{\mathrm{NS}}=\frac{1}{2 \kappa_{10}^{2}} \int\left[R_{10}^{\mathrm{E}} * 1-\frac{1}{2} e^{-\Phi} H_{3} \wedge * H_{3}-\frac{1}{2} \mathrm{~d} \Phi \wedge * \mathrm{~d} \Phi\right]
$$

and the part describing the dynamics of the R-R sector 1.22 transforms to

$$
S_{\mathrm{IIB}}^{\mathrm{R}}=-\frac{1}{4 \kappa_{10}^{2}} \int\left[e^{2 \Phi} F_{1} \wedge * F_{1}+e^{\Phi} \tilde{F}_{3} \wedge * \tilde{F}_{3}+\frac{1}{2} \tilde{F}_{5} \wedge * \tilde{F}_{5}+C_{4} \wedge H_{3} \wedge \tilde{F}_{3}\right] .
$$


If we now additionally define

$$
\tau \equiv C_{0}+i e^{-\Phi}, \quad G_{3} \equiv F_{3}-\tau H_{3},
$$

the type IIB action can be rewritten as

$$
\begin{aligned}
S_{\mathrm{IIB}}=\frac{1}{2 \kappa_{10}^{2}} \int\left[R_{10}^{\mathrm{E}} * 1-\frac{1}{2(\operatorname{Im} \tau)^{2}} \mathrm{~d} \tau\right. & \wedge * \mathrm{~d} \bar{\tau}-\frac{1}{2 \operatorname{Im} \tau} G_{3} \wedge * \bar{G}_{3} \\
& \left.-\frac{1}{4} \tilde{F}_{5} \wedge * \tilde{F}_{5}-\frac{i}{4 \operatorname{Im} \tau} C_{4} \wedge G_{3} \wedge \bar{G}_{3}\right] .
\end{aligned}
$$

In this form it can be easily checked that the action is invariant under the transformation

$$
\tau \rightarrow \frac{a \tau+b}{c \tau+d}, \quad\left(\begin{array}{c}
C_{2} \\
B_{2}
\end{array}\right) \rightarrow\left(\begin{array}{ll}
a & b \\
c & d
\end{array}\right)\left(\begin{array}{c}
C_{2} \\
B_{2}
\end{array}\right),
$$

where $a, b, c, d \in \mathbb{R}$ such that $a d-b c=1$. The action thus has an $S L(2, \mathbb{R})$ symmetry. In the quantum theory this symmetry gets broken to its discrete $S L(2, \mathbb{Z})$ subgroup. It is expected that this symmetry pertains in the full type IIB string theory. Note that it relates strong and weak coupling because the S-duality transformation

$$
S=\left(\begin{array}{cc}
0 & 1 \\
-1 & 0
\end{array}\right)
$$

sends $\tau \rightarrow-1 / \tau$ which means that $g_{s} \rightarrow 1 / g_{s}$ when $C_{0}=0$.

\subsubsection{Compactifications}

Spacetime in string theory and its corresponding low energy supergravity limit is ten-dimensional. Nature does not appear to have that many dimensions. Instead it seems to be four-dimensional: one time and three space dimensions. For string theory to describe our four-dimensional universe the extra six dimensions must be compact and small enough such that they are inaccessible to current experiments. Let's illustrate this with a long tube (see figure 1.2). If you view it from far away it looks just like a line. However as you zoom in closer you see that it also has a thickness, the circle is wrapping around. The zooming is equivalent to increasing the energy of the experiment. When the extra dimensions cannot be probed by experiment we can derive an effective lower dimensional theory. This process is called compactification and is the topic of this section. We start by explaining how to reduce on a circle, since this is the least complicated reduction one can do but nevertheless illustrates the important features. In addition we will make use of 
such a circle reduction at several points in this thesis. Having introduced how to compactify on a circle we will discuss compactifying supergravity on more general manifolds, namely Calabi-Yau threefolds.

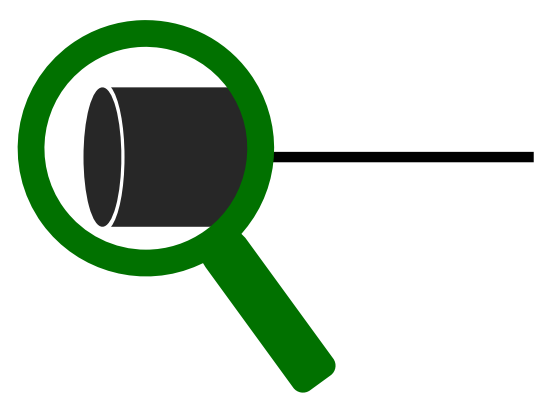

Figure 1.2: The idea of small compact dimensions. From far away a long tube looks like a line. Only when zooming in one discovers it is two-dimensional.

\subsubsection{Circle compactifications}

We discuss two kinds of circle compactification. We start by considering the reduction of a complex scalar in a background that contains a circle. This example mainly serves to illustrate the salient features of compactification. Following this we discuss what happens when gravity is put on a circle. The latter reduction is a very old idea by T. Kaluza and O. Klein developed in the 1920s in order to unify gravity and electromagnetism. They studied five-dimensional gravity, in which one dimension is compactified on a circle with very small radius. The five-dimensional theory only contains gravity but leads to an effective four-dimensional theory of gravity and an electromagnetic $U(1)$ symmetry when the radius of the circle is small enough, or equivalently for low-enough energies. This particular unification attempt failed although the ideas of extra dimensions stayed and play a huge role in string theory as we will see. Circle compactifications as well as other compactifications are generally referred to as Kaluza-Klein reductions.

A complex scalar. The main idea of a compactification can be illustrated by putting a complex massless scalar $\hat{\phi}$ on a circle. More precisely, let the $(D+1)$ dimensional background be given by $M_{D+1}=M_{D} \times \mathrm{S}^{1}$ such that the action for the scalar field reads

$$
S_{D+1}=\int_{M_{D+1}} \mathrm{~d} \hat{\phi} \wedge \hat{*} \mathrm{~d} \overline{\hat{\phi}} .
$$


The metric on $M_{D+1}$ is taken to be

$$
\mathrm{d} \hat{s}^{2}=\mathrm{d} s^{2}(x)+r^{2} \mathrm{~d} y^{2}, \quad y=y+2 \pi,
$$

where $\mathrm{d} s^{2}(x)$ is the metric on $M_{D}$ with coordinates $x, y$ is the coordinate along the circle and $r$ is the radius of this circle. Note that $\hat{\phi}$ has to be periodic in $y$, i.e. $\hat{\phi}(x, y)=\hat{\phi}(x, y+2 \pi)$ such that we can expand $\hat{\phi}$ in Fourier modes as

$$
\hat{\phi}(x, y)=\sum_{n \in \mathbb{Z}} \phi_{n}(x) e^{i n y}
$$

The action in terms of these lower dimensional fields becomes

$$
S_{D}=2 \pi r \int_{M_{D}} \sum_{n \in \mathbb{Z}}\left[\mathrm{d} \phi_{n} \wedge * \mathrm{~d} \bar{\phi}_{n}+\left(\frac{n}{r}\right)^{2} \phi_{n} \bar{\phi}_{n} * 1\right] .
$$

The $(D+1)$-dimensional action $\sqrt{1.33}$ is thus equivalent to a $D$-dimensional action 1.36 with an infinite number of complex scalars $\phi_{n}$ that have masses $n / r$. These scalars are also called the Kaluza-Klein modes of $\hat{\phi}$. Note that the massless wave equation splits into two pieces: $0=\partial_{\mu} \partial^{\mu} \hat{\phi}=\partial_{i} \partial^{i} \hat{\phi}+\frac{1}{r^{2}} \partial_{y}^{2} \hat{\phi}$, where $\mu=0, \ldots, D$ and $i=0, \ldots, D-1$. Also note that $\hat{\phi}$ is expanded in eigenfunctions of $\partial_{y}^{2}$ with the resultant masses corresponding to the eigenvalues. At energies $E \ll r^{-1}$ the massive modes cannot be excited and one can integrate them out in order to obtain an effective theory in terms of the zero mode $\phi_{0}$. For low energies one thus finds that the $(D+1)$-dimensional theory is effectively given by the $D$-dimensional theory of a complex scalar with action

$$
S_{D}=2 \pi r \int_{M_{D}} \mathrm{~d} \phi_{0} \wedge * \mathrm{~d} \bar{\phi}_{0}
$$

In order to probe the extra dimension via the detection of the massive Kaluza-Klein modes one needs energies $E \sim r^{-1}$. In general one cannot just throw away the massive modes in the compactification process. They can still contribute to the effective theory via loop diagrams in which the massive modes run in the loop. In chapters 3 -5 we will see that the inclusion of these kinds of terms is in certain cases even crucial.

Gravity. In the previous example we have shown the key ingredients of a KaluzaKlein reduction on a circle by studying what happens with a complex scalar. We now discuss pure gravity on a circle. While the original Kaluza-Klein reduction 
went from five to four dimensions, we will again keep the dimension arbitrary. Therefore, the $(D+1)$-dimensional action we start with is given by

$$
S_{D+1}=\frac{1}{2 \kappa_{D+1}^{2}} \int R_{D+1} \hat{*} 1 .
$$

In the example of the complex scalar we explicitly showed all the massive modes one obtains in $D$ dimensions, here we restrict ourselves to the zero modes. These zero modes are given by the lower dimensional metric $g_{\mu \nu}$, a one-form $A^{0}$ which is also often referred to as the Kaluza-Klein gauge field, and a scalar $r$. In terms of these fields the $(D+1)$-dimensional metric can be written as

$$
\mathrm{d} \hat{s}^{2}=\mathrm{d} s^{2}(x)+r^{2}\left(\mathrm{~d} y-A^{0}\right)^{2} .
$$

The one-form thus corresponds to the $g_{i y}$ components of the metric and the scalar to the $g_{y y}$ component. We indeed have a $U(1)$ gauge symmetry in $D$ dimensions corresponding to higher dimensional coordinate reparametrizations. That is, we can reparametrize $\delta y=f(x)$ for an arbitrary function $f$ such that $\delta A_{\mu}^{0}=\partial_{\mu} f$. Note that the scalar $r(x)$ corresponds to the radius of the circle. Integrating 1.38 over $y$ we obtain

$$
S_{D}=\frac{2 \pi}{2 \kappa_{D+1}^{2}} \int\left[r R_{D} * 1-\frac{1}{2} r^{3} F^{0} \wedge * F^{0}\right],
$$

where $F^{0}=\mathrm{d} A^{0}$. To get to Einstein frame, i.e. obtain terms with the correct normalization, we have to perform a field redefinition?

$$
g_{\mu \nu}^{\mathrm{E}}=\left(\frac{r}{r_{0}}\right)^{\frac{2}{D-2}} g_{\mu \nu}, \quad\left(A^{0}\right)^{\mathrm{E}}=r_{0}^{-\frac{1}{D-2}} A^{0},
$$

where we have introduced a scale $r_{0}$ in order to keep the metric dimensionless. With these rescalings and omitting the superscript $\mathrm{E}$ the action becomes

$$
S_{D}=\frac{1}{2 \kappa_{D}^{2}} \int\left[R_{D} * 1-\frac{D-1}{D-2} \frac{1}{r^{2}} \mathrm{~d} r \wedge * \mathrm{~d} r-\frac{1}{2} r^{\frac{2(D-1)}{D-2}} F^{0} \wedge * F^{0}\right],
$$

where the lower dimensional gravitational coupling constant is related to the higher dimensional constant via

$$
\kappa_{D}^{2}=\frac{\kappa_{D+1}^{2}}{2 \pi r_{0}} .
$$

We obtain a kinetic term for the radius, but no potential. This implies that the vacuum expectation value of the radius is arbitrary and this immediately poses ${ }^{9}$ Recall the discussion leading up to $[1.26]$. 
a problem for the validity of the lower dimensional description. As we have seen before, the massive Kaluza-Klein modes are only suppressed for sufficiently small energies, or equivalently for small-enough radii. However, if the radius is not fixed by a potential this is not guaranteed and we need to include these higher modes as well. Generating a potential in order to fix the vacuum expectation value of scalars describing the sizes of the extra dimensions is also necessary for higher dimensional compactifications and can generically be done by introducing extra fields in the higher dimensional theory.

\subsubsection{Calabi-Yau compactifications}

We now turn to a discussion of compactifications on a higher dimensional generalization of the circle. These are necessary in order for the ten-dimensional theories to describe four-dimensional physics. For now we focus on geometries of the form $\mathbb{R}^{1,3} \times M_{6}$, where $M_{6}$ is compact and such that part of the supersymmetry is preserved in the resulting theory on $\mathbb{R}^{1,3}$. Preserving supersymmetry (and only breaking it by some other mechanism) was initially motivated because it is a way to solve some of the problems with the Standard Model, e.g. the hierarchy problem. However, since experiments at the Large Hadron Collider have so far not found evidence for supersymmetry, the preservation of supersymmetry here is merely motivated by the computational control it provides. Indeed as we will see in sections 1.3 and 1.4 supersymmetry is an important ingredient in matching the microscopic and macroscopic entropy computations. Even if the $4 \mathrm{~d}$ supersymmetric physics does not describe nature, we still might hope to take away important lessons which we can apply to scenarios more reminiscent of nature. As we shall demonstrate, one such lesson is that black hole entropy can be computed by a counting of the microstates.

When fluxes are turned-off, preservation of supersymmetry in four dimensions requires the existence of a covariantly constant spinor on $M_{6}$. This is equivalent to the six-dimensional manifold having a subgroup of $S U(3)$ as restricted holonomy group. The restricted holonomy group is generated by parallel transport along contractible paths. Having this restricted holonomy group is in turn equivalent to $M_{6}$ having a Ricci-flat Kähler metric. However, it is rather hard to explicitly construct metrics with (a subgroup of) $S U(3)$ restricted holonomy. In fact apart from the torus, no compact examples have ever been constructed 10 We therefore need a better characterization of manifolds with such restricted holonomy groups.

\footnotetext{
${ }^{10}$ There are explicit metrics with $S U(3)$ holonomy for non-compact manifolds as we briefly discuss in section 1.4
} 
First note that Kähler manifolds admitting a Ricci-flat metric have a vanishing first Chern class, i.e. $c_{1}\left(M_{6}\right)=0$. Then such a characterization is provided by the Calabi-Yau theorem. Conjectured by E. Calabi and proven by S.T. Yau, it implies (among other results) that any compact Kähler manifold with vanishing first Chern class admits a unique Ricci-flat Kähler metric. The proof is existence based and does not explain how to construct such a metric. The beauty is that we do not need to construct a metric to perform the compactification. Manifolds $M_{6}$ with the right properties are thus given by compact Kähler manifolds with vanishing first Chern class. The easiest example is provided by the already mentioned six-torus $\mathrm{T}^{6}$ which is the higher dimensional generalization of the circle and has trivial holonomy. With this manifold maximal supersymmetry is preserved in 4d. A manifold that we will encounter in chapter 5 which has $S U(2)$ holonomy is given by $\mathrm{T}^{2} \times \mathrm{K} 3$ and breaks half of the supersymmetry. Here K3 is defined as a 4 d compact, simply connected Kähler manifold with restricted holonomy group $S U(2)$. The most general $6 \mathrm{~d}$ manifold of the kind above has $S U(3)$ as holonomy group and, as is commonly done in the literature, we refer to these manifolds as Calabi-Yau and denote them as $\mathrm{CY}_{3}$. General Calabi-Yau $n$-folds are then defined as compact Kähler manifolds of complex dimension $n$ with $S U(n)$ as restricted holonomy group.

As before, we would like to determine the lower dimensional massless fields upon compactifying on the Calabi-Yau threefold. This proceeds similarly to the circle example. One thus expands a generic 10d field around their vacuum expectation value: $\phi_{i}=\left\langle\phi_{i}\right\rangle+\varphi_{i}$. We substitute this into the equations of motion and keep only linearized terms in $\varphi_{i}$. After a possible gauge fixing we find that the equation of motion splits as

$$
\mathcal{O}_{4} \varphi_{i}+\mathcal{O}_{\mathrm{CY}} \varphi_{i}=0
$$

where $\mathcal{O}$ represents some operator. We then expand $\varphi_{i}$ in eigenfunctions of $\mathcal{O}_{\mathrm{CY}}$ and the corresponding eigenvalues correspond to the masses of the $4 \mathrm{~d}$ fields. Since we are interested in the massless fields in $4 \mathrm{~d}$ we need to consider the zero modes of $\mathcal{O}_{\mathrm{CY}}$. When we have a $p$-form $C_{p}$, the linearized equation of motion is given by $\mathrm{d} * \mathrm{~d} C_{p}=0$. We can gauge fix such that $\delta C_{p}=0$ where $\delta=* \mathrm{~d} *$ is the codifferential. For the gauge-fixed $p$-form the equation of motion can be rewritten using the Laplacian $\Delta=d \delta+\delta d$ as

$$
0=\Delta_{10} C_{p}=\Delta_{4} C_{p}+\Delta_{\mathrm{CY}} C_{p} .
$$

The lower dimensional forms with zero mass correspond to harmonic forms $\omega$ defined by $\Delta_{\mathrm{CY}} \omega=0$. These are in a one-to-one correspondence with the De Rham cohomology groups $H^{m}\left(\mathrm{CY}_{3}\right)$ which count harmonic $m$-forms. The lower dimensional 
field content is thus determined by the topological data of the compactification manifold. In particular the dimensions of the various De Rham cohomology groups, given by the Betti numbers $b^{m}$, will correspond to the number of different fields of a particular type in the lower dimensional theory.

Despite the lack of a metric we can compute these numbers by using that the Calabi-Yau threefold is Kähler and thus complex. This implies that its De Rham cohomology groups split as

$$
H^{m}\left(\mathrm{CY}_{3}\right)=\bigoplus_{p=0}^{m} H^{m-p, p}\left(\mathrm{CY}_{3}\right)
$$

where $H^{m-p, p}\left(\mathrm{CY}_{3}\right)$ counts harmonic forms with $m-p$ holomorphic and $p$ antiholomorphic indices. We thus have that

$$
b^{m}=\sum_{p=0}^{m} h^{m-p, p},
$$

where the Hodge numbers $h^{p, q}$ are the dimensions of the spaces $H^{p, q}\left(\mathrm{CY}_{3}\right)$. Note that on a Calabi-Yau threefold there are in principle 16 different Hodge numbers, however the majority of these are uniquely fixed. Assuming the $\mathrm{CY}_{3}$ to be connected, the zeroth Betti number $b^{0}=1$. From 1.47 it then follows that $h^{0,0}=b^{0}=1$. Complex conjugation implies that $h^{p, q}=h^{q, p}$ and from Hodge duality one finds that $h^{p, q}=h^{3-p, 3-q}$. Additionally having $S U(3)$ as holonomy group implies that $h^{m, 0}=0$ for $0<m<3$ and that $h^{3,0}=1$. With all these conditions the Hodge numbers for a Calabi-Yau threefold are given by the Hodge diamond

$$
\begin{aligned}
& h^{0,0} \\
& h^{1,0} \quad h^{0,1} \\
& h^{2,0} \quad h^{1,1} \quad h^{0,2}
\end{aligned}
$$

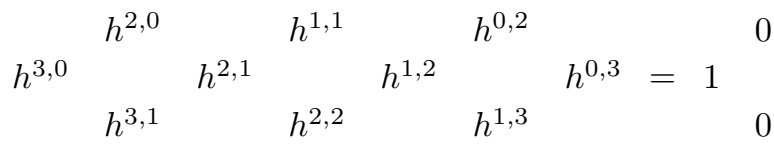

$$
\begin{aligned}
& h^{3,2} \quad h^{2,3}
\end{aligned}
$$

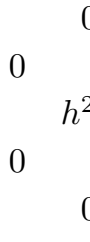

$$
\begin{aligned}
& 0 \quad 0
\end{aligned}
$$

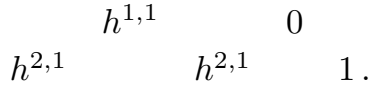

$$
\begin{aligned}
& 0 \\
& 1
\end{aligned}
$$

We see that there are only two independent Hodge numbers left where $h^{2,1} \geq 0$ and $h^{1,1} \geq 1$ since $\mathrm{CY}_{3}$ is Kähler. One can easily generalize the relations above to Calabi-Yau $n$-folds by just replacing every 3 by $n$. In particular one can derive the 
Hodge numbers of K3 which we will need in chapter 5 .

\begin{tabular}{|c|c|c|c|c|c|c|}
\hline \multicolumn{3}{|c|}{$h^{0,0}$} & & & \multicolumn{2}{|c|}{1} \\
\hline & $h^{1,0}$ & $h^{0,1}$ & & & 0 & 0 \\
\hline \multirow[t]{3}{*}{$h^{2,0}$} & & & $h^{0,2}$ & $=1$ & & \\
\hline & $h^{2,1}$ & $h^{1,2}$ & & & 0 & 0 \\
\hline & & & & & & \\
\hline
\end{tabular}

To determine the value of $h^{1,1}$ one has to use some additional results for $4 \mathrm{~d}$ simply-connected Calabi-Yau manifolds.

We can now use the Hodge numbers 1.48 to determine the 4d (bosonic) field content when type II theory is compactified on a Calabi-Yau threefold 11 For instance, we can expand the Kalb-Ramond field of the type II theories as $B_{2}=$ $b_{2}^{0} \omega_{0}+b_{0}^{i} \omega_{i}$, where $\omega_{0}$ is the only harmonic scalar since $h^{0,0}=1$ and $\omega_{i}$ for $i=1, \ldots, h^{1,1}$ are the harmonic two-forms. Therefore $B_{2}$ gives a four-dimensional two-form $b_{2}^{0}$ and $h^{1,1}$ real scalars $b_{0}^{i}$ in the compactified theory. In four dimensions the two-form $b_{2}^{0}$ can be dualized into a scalar. Therefore, $B_{2}$ leads to $h^{1,1}+1$ real scalars. In the same spirit we find that the reduction of the dilaton and axion both result in one scalar, the one-form $C_{1}$ gives a vector field, the two-form $C_{2} h^{1,1}+1$ real scalars, the three-form $2+2 h^{2,1}$ real scalars and $h^{1,1}$ vector fields and finally the four-form gives $h^{1,1}$ real scalars and $1+h^{2,1}$ vectors. For the reduction of $C_{4}$ we used the self-duality of $F_{5}$. Studying the massless modes coming from the $10 \mathrm{~d}$ metric is slightly more involved but one has to follow the procedure as outlined above. This results in a $4 \mathrm{~d}$ metric together with $h^{1,1}$ real scalars corresponding to deformations of the Kähler form and $h^{2,1}$ complex scalars corresponding to deformations of the complex structure. The bosonic fields resulting from the reduction of one of the type II theories together with the fermionic field content resulting from reducing the $10 \mathrm{~d}$ fermions group into $4 \mathrm{~d} \mathcal{N}=2$ supermultiplets.

We end this subsection with two general comments. Firstly, for the $4 \mathrm{~d}$ theory to be an effective description we need energies $E \ll L^{-1}$, where $L=\operatorname{vol}\left(\mathrm{CY}_{3}\right)^{1 / 6}$ is the length scale characterizing the size of the Calabi-Yau threefold. This does not imply that $L$ can be taken arbitrarily small since we should also be able to safely ignore stringy effects. Therefore we need $L \gg \ell_{s}$. Secondly, there are a lot of possible vacua for the theory and there is no mechanism known to determine the correct one. Including expectation values for gauge fields, one finds the infamous estimate $\mathcal{O}\left(10^{500}\right)$ for the number of type IIB vacua. All of these lead to different $4 \mathrm{~d}$ theories and one can wonder whether or not every consistent effective $4 \mathrm{~d}$ theory can

\footnotetext{
${ }^{11}$ See section 5.2 .1 for the $6 \mathrm{~d}$ field content when reducing type IIB supergravity on K3.
} 
be recovered. If this were true, string theory loses its ability to make predictions. However, in recent years much work has appeared conjecturing criteria for effective field theories to obey in order for them to be UV-complete within quantum gravity, usually assumed string theory. Effective $4 \mathrm{~d}$ theories not satisfying these criteria belong to the so-called Swampland. See [31,32] for reviews of the Swampland program.

\subsubsection{Branes}

Apart from strings the type II theories and M-theory contain higher dimensional objects called branes. They will be very important for the construction of black holes which we discuss in section 1.3. We first explain in section 1.2.4.1 how they arise in superstring theory. Then in section 1.2.4.2 we describe what branes one finds in M-theory. Lastly, in section 1.2.4.3 we discuss how branes can be described in supergravity.

\subsubsection{In superstring theory}

The perturbative type II spectrum only contains states arising from closed strings. However, as discussed previously there are also strings with the topology of a segment. The endpoints of these strings have to obey either Neumann or Dirichlet boundary conditions. If an endpoint satisfies Dirichlet boundary conditions in a certain direction, it is fixed to a particular point in space in that direction. Thus if the endpoint of a string has these boundary conditions in $9-p$ (spatial) directions, it is fixed to a $(p+1)$-dimensional surface called a $\mathrm{D} p$-brane. It took a few decades before it was realized that these higher dimensional surfaces can be stable objects on their own because they are charged under the R-R forms of the closed string spectrum. Therefore, which $\mathrm{D} p$-branes are stable depends on the string theory one considers. For type IIA string theory $p$ needs to be even, while for the type IIB $p$ needs to be odd. The branes are non-perturbative since they cannot be seen in the perturbative spectrum we discussed in section 1.2.1. To describe them one first has to quantize the open string.

The massless bosonic fields in the spectrum one obtains when both endpoints of the string are fixed to the same $\mathrm{D} p$-brane are $9-p$ real scalars $\phi^{I}$ and a $U(1)$ gauge field $A$ with field strength $F=\mathrm{d} A$. The scalars can be associated to the fluctuations of the $\mathrm{D} p$-brane in the transverse space. An infinite flat brane in flat space breaks the $10 \mathrm{~d}$ Lorentz group $S O(1,9) \rightarrow S O(1, p) \times S O(9-p)$, where $S O(1, p)$ is the Lorentz group on the worldvolume of the brane and $S O(9-p)$ is a global symmetry 
acting on the scalars. Additionally, this brane breaks half of the supersymmetry. In a similar way as we described in 1.2 .2 one can derive an effective action for the massless bosonic fields of the $\mathrm{D} p$-brane in the low-energy limit. This action consists of two pieces, the Dirac-Born-Infeld (DBI) action and a Chern-Simons action:

$$
\begin{aligned}
S_{\mathrm{brane}} & =S_{\mathrm{DBI}}+S_{\mathrm{CS}} \\
S_{\mathrm{DBI}} & =-T_{p} \int_{W_{p+1}} e^{-\Phi} \sqrt{-\operatorname{det}\left(g+B_{2}+2 \pi \alpha^{\prime} F\right)} \\
S_{\mathrm{CS}} & =T_{p} \int_{W_{p+1}}\left(\sum_{k} C_{k}\right) \wedge \exp \left(B_{2}+2 \pi \alpha^{\prime} F\right) \wedge \sqrt{\frac{\hat{A}\left(4 \pi^{2} \alpha^{\prime} \mathcal{R}_{T}\right)}{\hat{A}\left(4 \pi^{2} \alpha^{\prime} \mathcal{R}_{N}\right)}},
\end{aligned}
$$

where $W_{p+1}$ is the worldvolume of the $\mathrm{D} p$-brane and $T_{p}=2 \pi\left(4 \pi^{2} \alpha^{\prime}\right)^{-(p+1) / 2}$ is its tension. The fields $g, \Phi, B_{2}$ and $C_{k}$ in 1.50 are the fields from the closed string spectrum pulled-back to the brane's worldvolume. The sum over $k$ is over odd $1 \leq k \leq 9$ for type IIA and over even $0 \leq k \leq 10$ for type IIB ${ }^{12}$ The A-roof genus $\hat{A}$ can be expressed in terms of Pontryagin classes $p_{n}$ :

$$
\hat{A}(R)=1-\frac{1}{24} p_{1}+\ldots
$$

where

$$
p_{1}=-\frac{1}{2} \frac{1}{(2 \pi)^{2}} \operatorname{tr} \mathcal{R} \wedge \mathcal{R} .
$$

In the Chern-Simons part of the action $1.50 \mathcal{R}_{T}, \mathcal{R}_{N}$ refer to the curvature forms of respectively the tangent and normal bundle. We also find a term

$$
T_{p} \int_{W_{p+1}} C_{p+1} \subset S_{\mathrm{CS}}
$$

such that indeed the $\mathrm{D} p$-brane couples electrically to $C_{p+1}$. Note that the brane is also magnetically coupled to $C_{7-p}$.

For an infinite flat brane in flat space

$$
g_{a b}=\eta_{a b}+\frac{\partial X^{I}}{\partial \xi^{a}} \frac{\partial X^{J}}{\partial \xi^{b}} \delta_{I J}, \quad a=0, \ldots, p
$$

where $X^{I}(\xi)=2 \pi \alpha^{\prime} \phi^{I}(\xi)$ describe the transverse fluctuations. For small field strengths $F_{a b}$ and small gradients $\partial_{a} X^{I}$ the DBI action can be expanded. We find

$$
S_{\mathrm{DBI}}=-\left(2 \pi \alpha^{\prime}\right)^{2} T_{p} \int \mathrm{d}^{p+1} \xi e^{-\Phi}\left(\frac{1}{2} \partial_{a} \phi^{I} \partial^{a} \phi_{I}+\frac{1}{4} F_{a b} F^{a b}\right),
$$

\footnotetext{
${ }^{12}$ The field $C_{k}$ is via Hodge duality related to $C_{8-k}$.
} 
which is the bosonic part of maximally supersymmetric Yang-Mills theory with $U(1)$ gauge group and coupling

$$
g_{\mathrm{YM}}^{2}=\left(2 \pi \alpha^{\prime}\right)^{-2} \frac{g_{s}}{T_{p}}=g_{s}(2 \pi)^{p-2}\left(\alpha^{\prime}\right)^{\frac{p-3}{2}} .
$$

The theory is invariant under 16 supercharges because D-branes break half of the supersymmetry. When we do not just have one D-brane but a stack of $N$ D-branes, both endpoints of the string can end on each of the branes. The $U(1)$ symmetry generalizes to a $U(N)$ symmetry and instead of a perturbative expansion in $g_{s}$ we get an expansion in $g_{s} N$. Unfortunately, there is no closed form for the DBI action in the case of a non-abelian field strength $F$. However, we can again consider the limit of small field strengths. The action 1.55 can be generalized and becomes the bosonic part of the action of maximally supersymmetric Yang-Mills with gauge group $U(N)$.

So far we have been looking at branes which are charged under the R-R forms. We also have the Kalb-Ramond field $B_{2}$ which is electrically coupled to the fundamental string. As for the R-R forms it is also coupled magnetically to a brane which is called the NS5-brane.

We have seen that there are several symmetries connecting the theories. It is natural to ask what the fate of the branes is under these symmetries. Under T-duality, which connects the type II theories on a circle, a $\mathrm{D} p$-brane is sent to a $\mathrm{D}(p-1)$-brane if it wraps the circle and to a $D(p+1)$-brane if it is not wrapped. An NS5-brane is sent to an NS5-brane. The $S L(2, \mathbb{Z})$ symmetry 1.31 of type IIB requires a more thorough discussion. First of all it acts on $B_{2}$ and $C_{2}$. Therefore the fundamental string, which is electrically charged under $B_{2}$ is mapped to a $(p, q)$ string under the $S L(2, \mathbb{Z})$ element

$$
M=\left(\begin{array}{cc}
r & s \\
q & p
\end{array}\right), \quad r p-q s=1 .
$$

A $(p, q)$ string has $p$ units of charge under $B_{2}$ and $q$ units of charge under $C_{2}$ and is a non-perturbative object for $(p, q) \neq(1,0)$. In particular a $(0,1)$ string can be identified as the D1-string. Note that under the action of a generic $S L(2, \mathbb{Z})$ element (1.31) a $(p, q)$ string is sent to a $\left(p^{\prime}, q^{\prime}\right)$ string where

$$
\left(q^{\prime}, p^{\prime}\right)=(q, p)\left(\begin{array}{ll}
a & b \\
c & d
\end{array}\right) .
$$

The five-branes are magnetically charged under $B_{2}$ and $C_{2}$ which is why we also have $(p, q)$ five-branes. The four-form $C_{4}$ is invariant under this symmetry which is 
why D3-branes are invariant. The group does however act on $\tau$ of which the real part is given by $C_{0}$ under which D7-branes are magnetically charged. In analogy with the strings and five-branes a D7-brane gets sent to a $(p, q)$ seven-brane under the element (1.57). Since a $(1,0)$ string ends on a $(1,0)$ five-brane or a $(1,0)$ seven-brane, a $(p, q)$ string ends on a $(p, q)$ five-brane or a $(p, q)$ seven-brane.

Let's consider the seven-branes in more detail. We first examine what $\tau$ looks like in the presence of a D7-brane. Since the D7-brane has unit magnetic charge under $C_{0}$ we find that

$$
\oint_{\mathrm{S}^{1}} F_{1}=\oint_{\mathrm{S}^{1}} \mathrm{~d} C_{0}=1,
$$

where $\mathrm{S}^{1}$ is a circle around the D7-brane in the transverse space $\mathbb{C}$, i.e. $C_{0} \rightarrow C_{0}+1$ when circling around the brane. Denote the complex coordinate of the transverse space by $z$ and suppose the brane is located at $z=z_{0}$. Supersymmetry of the D7-brane implies that $\bar{\partial} \tau=0$ away from the brane. The solution for $\tau$ close to $z_{0}$ is then given by

$$
\tau(z)=\frac{1}{2 \pi i} \log \left(\frac{z-z_{0}}{\lambda}\right),
$$

up to terms regular at $z_{0}$. Here $\lambda \in \mathbb{C}$. Note that near the brane $\tau \rightarrow i \infty$ such that $g_{s} \rightarrow 0$. Therefore this solution is reliable. The solution 1.60 already seems to indicate that a D7-brane has a long range effect and this is indeed what follows after a more careful study. In fact, generically this backreaction is so strong that the dimensions transverse to the brane have to be compact. Therefore the backreaction of this brane cannot be neglected, even at asymptotic distances and we cannot treat the brane as a probe on a background geometry. Conversely for lower dimensional branes we may use this probe approximation since the backreaction becomes negligible asymptotically far from the brane. Note that one also expects strongly coupled regions when $\left|z-z_{0}\right| \sim|\lambda|$ since there $g_{s} \rightarrow \infty$. In these regions the perturbative type IIB description breaks down.

The monodromy $\tau \rightarrow \tau+1$ as we go around the D7-brane can be understood using the $S L(2, \mathbb{Z})$ symmetry of type IIB. Indeed, when we act with the element

$$
T=\left(\begin{array}{ll}
1 & 1 \\
0 & 1
\end{array}\right)
$$

we find that $\tau \sim \tau+1$ under $S L(2, \mathbb{Z})$. Of course from 1.31 it follows that for consistency we also have to act on $B_{2}$ and $C_{2}$. So when a $(p, q)$ string moves around the D7-brane, it comes back as a $(p+q, q)$ string. So far we considered monodromies around a $(1,0) 7$-brane. Since a general $(p, q) 7$-brane is obtained from a $(1,0)$ 
7 -brane by acting with the element 1.57 the $S L(2, \mathbb{Z})$ monodromy matrix for circling around a $(p, q)$ 7-brane is given by

$$
M_{(p, q)}=M^{-1} T M=\left(\begin{array}{cc}
1+p q & p^{2} \\
-q^{2} & 1-p q
\end{array}\right) .
$$

Here we first transformed the $(p, q)$ 7-brane to a D7-brane for which we know the monodromy matrix. After performing the monodromy transformation we transform back to the $(p, q)$ 7-brane frame. Note that only $\left(p^{\prime}, q^{\prime}\right)$ strings such that $p q^{\prime}=q p^{\prime}$ come back as the same string after circling around a $(p, q)$ seven-brane. A $(p, q)$ and a $\left(p^{\prime}, q^{\prime}\right)$ 7-brane such that $p q^{\prime}=q p^{\prime}$ have commuting monodromy matrices and are called mutually local. Seven-branes that do not satisfy this condition are called mutually non-local. Type IIB with 7-branes poses a problem for the perturbative framework we have been working in so far. A single $(p, q)$ 7-brane can always be transformed into a D7-brane such that perturbation theory can be used. However, once there are multiple mutually non-local 7-branes this is not possible anymore. When we loop a fundamental string around a 7-brane mutually non-local with respect to a D7-brane we get back a non-perturbative string. These non-local branes are often necessary, e.g. when the space transverse to the D7-branes is compact one needs the total monodromy to vanish for consistency. This means that it is impossible to define a perturbative theory for fundamental strings in a globally well-defined way in the presence of D7-branes. Luckily there is an elegant reformulation of the problem and this will be the topic of section 1.2 .5 .

\subsubsection{In M-theory}

As discussed in section 1.2.1 M-theory is related to type IIA by a circle reduction. The low-energy limit of M-theory is given by $11 \mathrm{~d}$ supergravity with action (1.25). Its bosonic field content consists of a metric and a three-from $A_{3}$. M-theory thus contains M2-branes and M5-branes that are respectively electrically and magnetically coupled to $A_{3}$. The number refers again to the number of spatial dimensions of the brane. Analogous to section 1.2 .3 one can derive how the $11 \mathrm{~d}$ fields are related to the $10 \mathrm{~d}$ field content upon compactification on the circle. The $11 \mathrm{~d}$ metric leads to the $10 \mathrm{~d}$ metric, the one-form $C_{1}$ and the dilaton. The $11 \mathrm{~d}$ three-form leads to the $10 \mathrm{~d}$ three-form $C_{3}$ and the two-form $B_{2}$. Therefore, the type IIA fundamental strings correspond to M2-branes wrapped on the circle. Similarly unwrapped M2-branes lead to D2-branes. M5-branes yield NS5-branes when they are not wrapped and D4-branes when they are wrapped on the circle. The type IIA D0-branes correspond to Kaluza-Klein states of the 11d theory and are electrically charged with respect 
to the Kaluza-Klein gauge field $C_{1}$. The D6-branes are the magnetic dual of the D0-branes which is why they correspond to Kaluza-Klein monopoles of the compactified theory. Such a monopole is a gravitational configuration in $11 \mathrm{~d}$ that looks like a magnetic monopole in $10 \mathrm{~d}$.

\subsubsection{In supergravity}

During the second superstring revolution in the 1990s it was revealed that $\mathrm{D} p$-branes and higher dimensional solutions to the supergravity equations of motion, called $p$-branes, are one and the same thing. We shall give the $p$-brane solutions and motivate their connection with the D-branes discussed previously in this section. In string frame the relevant $p$-branes for $p<7$ are given by

$$
\begin{aligned}
\mathrm{d} s^{2} & =H_{p}^{-1 / 2}\left[-\mathrm{d} x_{0}^{2}+\mathrm{d} x_{1}^{2}+\ldots+\mathrm{d} x_{p}^{2}\right]+H_{p}^{1 / 2}\left[\mathrm{~d} r^{2}+r^{2} \mathrm{~d} \Omega_{8-p}^{2}\right], \\
e^{\Phi} & =g_{s} H_{p}^{\frac{3-p}{4}} \\
C_{p+1} & =\left(H_{p}^{-1}-1\right) g_{s}^{-1} \mathrm{~d} x^{0} \wedge \ldots \wedge \mathrm{d} x^{p},
\end{aligned}
$$

where we have used spherical coordinates to describe the space transverse to the brane and the harmonic function $H_{p}$ is equal to

$$
H_{p}=1+\frac{Q_{p}}{r^{7-p}}, \quad Q_{p}=2^{5-p} \pi^{\frac{5-p}{2}} \Gamma\left(\frac{7-p}{2}\right) g_{s} \alpha^{\prime \frac{7-p}{2}} N_{p} .
$$

All other fields vanish in the solution. More general $p$-brane solutions exist, however the solutions 1.63 are the relevant ones for comparison with $\mathrm{D} p$-branes since they have the same symmetries, part of which is broken by the more general solutions. In particular they preserve half of the supersymmetry. Additionally the solution 1.63 and a $\mathrm{D} p$-brane have the same amplitudes for low energy scattering, emission and absorption processes. The presented $p$-branes carry R-R charges $Q_{p}$ which are quantized in terms of the number of D-branes $N_{p}$. The solution 1.63 is only valid for $g_{s} N_{p} \gg 1$ because otherwise curvatures become so large that $\alpha^{\prime}$-corrections become relevant. Since supergravity as a theory is only valid in the limit that $g_{s} \ll 1$, this implies that the presented solution is only a good description of D-branes in the limit of large $N_{p}$. It is clear that when $r \rightarrow \infty$ the solution 1.63 becomes flat, this is other evidence that we can indeed treat $\mathrm{D} p$-branes for $p<7$ with the probe approximation. When $p=7$, the function $H_{p}$ becomes logarithmic in $r$ such that the solution is not asymptotically flat anymore. This again signals that the backreaction of D7-branes cannot be neglected at asymptotic distances. 


\subsubsection{F-theory}

We have seen that type IIB string theory with 7-branes is inherently non-perturbative and that it can be difficult to keep track of all the $S L(2, \mathbb{Z})$ monodromies. In 1996 a framework called F-theory was proposed to deal with these difficulties in a consistent way $33{ }^{13}$ The idea is to identify the $S L(2, \mathbb{Z})$ symmetry with the $S L(2, \mathbb{Z})$ action on the complex structure of an auxiliary two-torus $\mathrm{T}^{2}$. To make this more specific we recall that a two-torus can be defined as the quotient of $\mathbb{C}^{2}$ by a lattice, i.e.

$$
\mathrm{T}^{2}=\mathbb{C}^{2} /(\mathbb{Z} \oplus \tau \mathbb{Z})
$$

where $\tau \in \mathbb{C}, \operatorname{Im} \tau>0$. The action of $S L(2, \mathbb{Z})$ on the complex structure modulus $\tau$ as in 1.31 will leave the torus invariant and therefore we can identify $\tau$ with the axio-dilaton of type IIB. The idea is thus to assign a torus $\mathrm{T}^{2}(\tau)$, specified by its complex structure modulus $\tau$, to every point of the 10d type IIB geometry which is why we can view F-theory as a $12 \mathrm{~d}$ framework. As we have seen, in the presence of 7-branes $\tau$ has a non-trivial profile and it diverges at the locations of these branes.

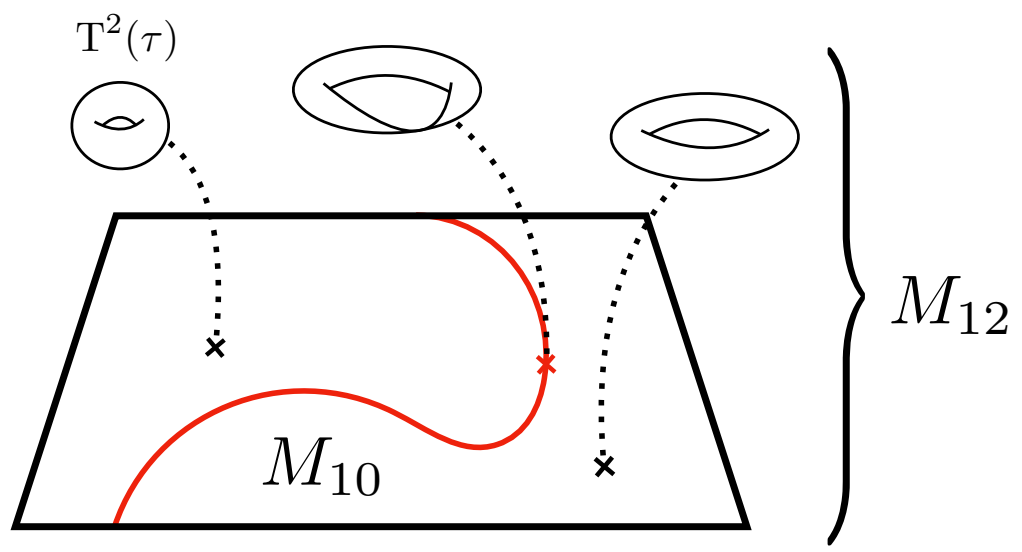

Figure 1.3: Illustration of an elliptic fibration $M_{12}$. At each point of the base manifold $M_{10}$ is attached a two-torus $\mathrm{T}^{2}$. This torus can vary in complex structure modulus $\tau$ and hence its shape for different points in $M_{10}$. At the location of 7-branes in $M_{10}$ (red line) the torus degenerates.

\footnotetext{
${ }^{13}$ We again keep the discussion relatively concise, for an in-depth introduction to F-theory we refer to the lecture notes 34,36 .
} 
Therefore, the $12 \mathrm{~d}$ geometry is an elliptic fibration

$$
\mathrm{T}^{2} \hookrightarrow M_{12} \rightarrow M_{10}
$$

where we can define a projection map $\pi: M_{12} \rightarrow M_{10}$ that projects the torus at a specific point on the base $M_{10}$ to that point. The fiber degenerates at the location of 7-branes. The elliptic fibration as depicted in figure 1.3 automatically keeps track of monodromies around singular points and is why F-theory provides a suitable framework to study type IIB string theory in a globally well-defined way.

We would like to stress that F-theory should be considered as merely a framework and not as a fundamental $12 \mathrm{~d}$ theory that when compactified on a torus leads to type IIB. First of all, there does not exist a supergravity theory in twelve dimensions with signature $(1,11)$ that would be the low-energy description of F-theory. Secondly, a torus reduction does not only give rise to just the complex structure modulus, but also to the modulus which fixes the overall size of the torus. There is no candidate for this modulus in the $10 \mathrm{~d}$ theory as there should be. Even without an interpretation as a fundamental theory one can still derive effective actions. To do this one can use a chain of dualities that expresses F-theory via a limit of M-theory. This is presented in figure 1.4 and schematically works as follows. Consider M-theory on $M_{9} \times \mathrm{S}_{T}^{1} \times \mathrm{S}_{M}^{1}$, where $\mathrm{S}_{M}^{1}$ is the M-theory circle and we will use the circle $\mathrm{S}_{T}^{1}$ for a T-duality. The circles have radii $R_{T}$ and $R_{M}$, and $\tau$ is the complex structure modulus of the torus $\mathrm{S}_{T}^{1} \times \mathrm{S}_{M}^{1}$. We consider M-theory on this background in the limit that the volume of the torus goes to zero via the

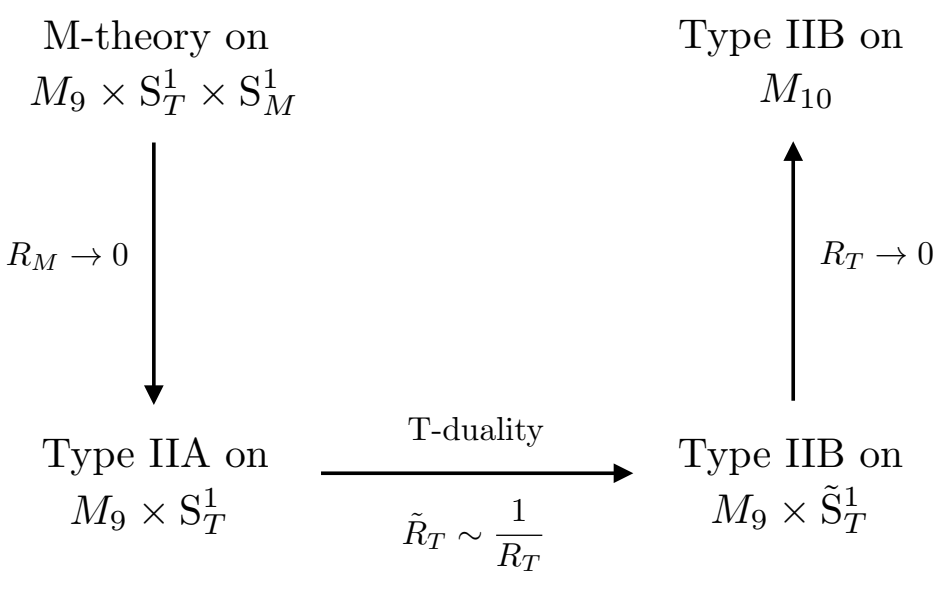

Figure 1.4: The duality chain that expresses F-theory via a limit of M-theory. 
following procedure. First one takes the radius $R_{M}$ to zero such that we get weakly coupled type IIA on $M_{9} \times \mathrm{S}_{T}^{1}$. After this we perform a T-duality along the remaining circle which results in type IIB on $M_{9} \times \tilde{\mathrm{S}}_{T}^{1}$ with the radius of the circle given by $\tilde{R}_{T} \sim 1 / R_{T}$. Taking $R_{T}$ to zero then results in 10d type IIB. When one follows $\tau$ through this chain of dualities one finds that it becomes the axio-dilaton of type IIB. Taking an elliptic fibration $\mathrm{T}^{2} \hookrightarrow M_{11} \rightarrow M_{9}$ and performing this chain of dualities fiber wise we end up with F-theory. We can now define F-theory as the type IIB theory dual to M-theory on the relevant elliptic fibration where the duality chain is as just described.

We will be mostly interested in compactifications of F-theory preserving some of the supersymmetry. When one considers a type IIB setting with spacetime filling 7-branes in an asymptotic geometry $\mathbb{R}^{1,9-2 n} \times B_{2 n}$, supersymmetry implies that $B_{2 n}$ has to be a compact Kähler manifold. This manifold is not Ricci-flat because of the long range effect of the 7-branes wrapping a divisor in $B_{2 n}$. The corresponding F-theory setting is thus compactified on an elliptic fibration with base $B_{2 n}$. At the location of 7 -branes in the base the fiber degenerates. These fiber singularities may or may not yield singularities of the whole space. Following the chain of dualities we presented above, supersymmetry preservation implies that M-theory needs to be compactified on an elliptically fibered Calabi-Yau manifold. When the compactification manifold on the F-theory side is singular one has to resolve these singularities in order for the compactification to work. The singular manifold is recovered in the limit of vanishing fiber volume.

An advantage of F-theory with respect to the perturbative type IIB framework is that physics questions can be translated to questions about the geometric properties of the fibration. The study of elliptic fibrations is then intimately related to the physics in lower dimensions resulting through compactifications with 7-branes. In particular recall that D-branes carry a gauge group on their worldvolume. In F-theory fiber degenerations at the location of 7-branes may yield singularities of the whole compactification space. These singularities correspond to stacks of 7-branes which admit a non-abelian gauge group on their worldvolume. The possible gauge groups can then be determined by studying the different singularities that can occur. It turns out that in F-theory one can even construct exceptional gauge groups which are not realized in perturbative type IIB.

Using the chain of dualities in figure 1.4 one can derive lower dimensional effective actions for F-theory on elliptically fibered Calabi-Yau $n$-folds $\mathrm{CY}_{n}$. One compactifies M-theory on the resolution $\mathrm{CY}_{n}$ and then takes the zero fiber-volume limit which decompactifies a circle. However, the latter limit brings M-theory to a regime 
where non-perturbative effects become important and one loses computational control. Therefore, in practice one can derive an effective $(12-2 n)$-dimensional action by taking the general $(12-2 n)$-supergravity theory with the correct supersymmetry and compactifying that theory on a circle. In this way one obtains two actions in $11-2 n$ dimensions, one from the M-theory reduction and one from the circle reduction. By comparing these two theories one derives what the correct $(12-2 n)$-dimensional effective action is in terms of data of the elliptically fibered Calabi-Yau $n$-fold. Note that this comparison takes place in the Coulomb branch of the theory because resolving the singular manifold on the M-theory side breaks the non-abelian gauge groups to their Cartans. In chapters 3 and 4 we will use F-theory compactifications on elliptically fibered threefolds. This leads to an $\mathcal{N}=(1,0)$ supergravity theory in six dimensions as derived in [37. This theory has eight real supercharges which is indeed equal to the number of supercharges one finds when compactifying M-theory on a Calabi-Yau threefold. The $\mathcal{N}=(1,0)$ theory has four types of multiplets, namely the gravity multiplet, tensor multiplets, vector multiplets and hypermultiplets (which can be charged). We discuss the relevant theories further in section 3.3.1

\subsection{Black holes in string theory}

Now that we have seen what string theory entails, let us find out how one can count microstates of black holes within this theory. Black holes in string theory can be made from D-branes wrapped in the compactified spacelike dimensions. The microstates of the black hole then correspond to the different ways open strings between those branes can vibrate. They are thus captured by the worldvolume theory of the branes. The challenge is to count them. This was first performed in 38 for type IIB string theory on $\mathrm{S}^{1} \times \mathrm{T}^{4}$ where the black hole is made out of D1-branes wrapping $\mathrm{S}^{1}$ and D5-branes wrapping the whole compact space. Additionally the system carries momentum along $\mathrm{S}^{1}$. After compactification this system corresponds to a five-dimensional black hole. In section 1.3 .1 we discuss this solution in more detail and use it to illustrate some of the salient features of the black holes we consider in this thesis. Afterwards we show in section 1.3 .2 how one can count the microstates of black holes in string theory using the worldvolume theory of the branes. The ingredients that we use are that the black holes admit a higher dimensional black string picture and moreover that they preserve some supersymmetry. 


\subsubsection{Black hole solutions as compactified D-brane solutions}

In this thesis we will be interested in black holes that arise from wrapped D3-branes. Nevertheless it is instructive to consider the D1-D5 black hole studied in 38,39 in more detail to exemplify some of the notable features of black holes in string theory 14 The corresponding 10d supergravity solution is in string frame given by

$$
\begin{aligned}
\mathrm{d} s_{10}^{2}= & H_{1}^{-\frac{1}{2}} H_{5}^{-\frac{1}{2}}\left[-\mathrm{d} t^{2}+\mathrm{d} y^{2}+K(\mathrm{~d} t-\mathrm{d} y)^{2}\right]+H_{1}^{\frac{1}{2}} H_{5}^{-\frac{1}{2}} \mathrm{~d} s_{\mathrm{T}^{4}}^{2} \\
& +H_{1}^{\frac{1}{2}} H_{5}^{\frac{1}{2}}\left[\mathrm{~d} r^{2}+r^{2} \mathrm{~d} \Omega_{3}^{2}\right], \\
e^{\Phi}= & g_{s} H_{1}^{\frac{1}{2}} H_{5}^{-\frac{1}{2}}, \\
C_{2}= & \left(H_{1}^{-1}-1\right) \mathrm{d} t \wedge \mathrm{d} y+Q_{5} \cos ^{2} \theta \mathrm{d} \varphi_{1} \wedge \mathrm{d} \varphi_{2},
\end{aligned}
$$

where $y$ is the circle coordinate, $\mathrm{d} s_{\mathrm{T}^{4}}^{2}$ is the flat metric on the four-torus, $\mathrm{d} \Omega_{3}^{2}=$ $\mathrm{d} \theta^{2}+\sin ^{2} \theta \mathrm{d} \varphi_{1}^{2}+\cos ^{2} \theta \mathrm{d} \varphi_{2}^{2}$ is the metric on $\mathrm{S}^{3}$ where $0<\theta<\frac{\pi}{2}$ and $0<\varphi_{1,2}<2 \pi$. Furthermore, the harmonic functions are given by

$$
H_{1}=1+\frac{Q_{1}}{r^{2}}, \quad H_{5}=1+\frac{Q_{5}}{r^{2}}, \quad K=\frac{Q_{n}}{r^{2}} .
$$

This solution is constructed from combining different $\mathrm{D} p$-brane solutions 1.63 and a pp-wave which is a generalization of a gravitational wave. Details on how this construction works can be found in e.g. [40]. It is clear that the two-form is coupled electrically to the D1-branes and magnetically to the D5-branes as it should be. The D1-branes, D5-branes and pp-wave each break one half of the supersymmetry, so the final solution preserves four supercharges. Let us now compactify on the $\mathrm{S}^{1} \times \mathrm{T}^{4}$ compact part of spacetime. Since there is no dependence on the torus directions, compactification of 1.67 to $6 \mathrm{~d}$ can be done by discarding the $\mathrm{T}^{4}$-coordinates. This results in a $6 \mathrm{~d}$ metric

$$
\begin{aligned}
\mathrm{d} s_{6}^{2}= & H_{1}^{-\frac{1}{2}} H_{5}^{-\frac{1}{2}}\left[-(1+K)^{-1} \mathrm{~d} t^{2}+(1+K)\left(\mathrm{d} y-K(1+K)^{-1} \mathrm{~d} t\right)^{2}\right] \\
& +H_{1}^{\frac{1}{2}} H_{5}^{\frac{1}{2}}\left[\mathrm{~d} r^{2}+r^{2} \mathrm{~d} \Omega_{3}^{2}\right],
\end{aligned}
$$

which we have rewritten in the form 1.39 to better facilitate the compactification to $5 \mathrm{~d}$. Using the prescription given in section 1.2.3.1 and in particular the ansatz 1.39 we find that the $5 \mathrm{~d}$ metric is given by

$$
\mathrm{d} s_{5}^{2}=-(1+K)^{-1} H_{1}^{-\frac{1}{2}} H_{5}^{-\frac{1}{2}} \mathrm{~d} t^{2}+H_{1}^{\frac{1}{2}} H_{5}^{\frac{1}{2}}\left[\mathrm{~d} r^{2}+r^{2} \mathrm{~d} \Omega_{3}^{2}\right] .
$$

\footnotetext{
${ }^{14}$ Note that this setting can be dualized to a black hole arising from wrapped D3-branes by T-dualizing along two circles in the $\mathrm{T}^{4}$. This results however in two intersecting stacks of D3-branes instead of one stack as we will use in chapters 35 of this thesis.
} 
We would now like to point out a couple of features of the five-dimensional solution. To do so we first rewrite it in Einstein frame using 1.26 with $\Phi \rightarrow \Phi_{5}-\left\langle\Phi_{5}\right\rangle,{ }^{15}$ where the five-dimensional dilaton is given by

$$
e^{-2 \Phi_{5}}=(1+K)^{\frac{1}{2}} H_{1}^{\frac{3}{4}} H_{5}^{-\frac{5}{4}} e^{-2 \Phi}=g_{s}^{-2}(1+K)^{\frac{1}{2}} H_{1}^{-\frac{1}{4}} H_{5}^{-\frac{1}{4}} .
$$

This results in

$$
\mathrm{d} s_{5}^{2}=-\left[(1+K) H_{1} H_{5}\right]^{-\frac{2}{3}} \mathrm{~d} t^{2}+\left[(1+K) H_{1} H_{5}\right]^{\frac{1}{3}}\left[\mathrm{~d} r^{2}+r^{2} \mathrm{~d} \Omega_{3}^{2}\right] .
$$

The metric 1.72 is a $5 \mathrm{~d}$ black hole with event horizon at $r=0$. Close to the horizon one obtains the metric

$$
\mathrm{d} s_{5}^{2}=\frac{\left(Q_{1} Q_{5} Q_{n}\right)^{\frac{1}{3}}}{4 \rho^{2}}\left[-\mathrm{d} t^{\prime 2}+\mathrm{d} \rho^{2}\right]+\left(Q_{1} Q_{5} Q_{n}\right)^{\frac{1}{3}} \mathrm{~d} \Omega_{3}^{2},
$$

where we have introduced new coordinates $t=\frac{1}{2} \sqrt{Q_{1} Q_{5} Q_{n}} t^{\prime}$ and $r=1 / \sqrt{\rho}$. The area of the horizon is thus given by

$$
A=2 \pi^{2} \sqrt{Q_{1} Q_{5} Q_{n}} .
$$

The macroscopic charges are expressed in terms of the number of D1-branes $N_{1}$, D5-branes $N_{5}$ and units of momentum $n$ via $Q_{1}=c_{1} N_{1}, Q_{5}=c_{5} N_{5}$ and $Q_{n}=c_{n} n$. The numbers $c_{1,5, n}$ can be computed, see e.g. [41], and as it turns out

$$
c_{1} c_{5} c_{n}=\frac{16\left(G^{(5)}\right)^{2}}{\pi^{2}},
$$

where $G^{(5)}$ is the five-dimensional gravitational constant. The entropy of the $5 \mathrm{~d}$ black hole is thus given by

$$
S=\frac{A}{4 G^{(5)}}=2 \pi \sqrt{N_{1} N_{5} n} .
$$

Note that 1.73 is the metric of $\mathrm{AdS}_{2} \times \mathrm{S}^{3}$. This $\mathrm{AdS}_{2}$ factor in the near-horizon geometry is a generic feature of supersymmetric black holes. Supersymmetric black strings such as 1.69 have an $\mathrm{AdS}_{3}$ factor in their near-horizon geometry. One might remember from a course in general relativity that one finds such a near-horizon geometry in 4d for the extremal Reissner-Nordström black hole. The metric of this black hole is given by

$$
\mathrm{d} s_{4}^{2}=-\left(1-\frac{G M}{r}\right)^{2} \mathrm{~d} t^{2}+\left(1-\frac{2 G M}{r}\right)^{-2} \mathrm{~d} r^{2}+r^{2} \mathrm{~d} \Omega_{2}^{2},
$$

\footnotetext{
$\overline{15}$ Technically this transformation brings us to the modified Einstein frame which is chosen such that the metric at infinity is normalized to be Minkowski.
} 
where $\mathrm{d} \Omega_{2}^{2}$ is the metric on $\mathrm{S}^{2}$. It is the generalization of the Schwarzschild black hole (1.1) to a black hole that is also charged in such a way that the mass is equal to the charge in appropriate units. Recall that for the string theory solutions the event horizon is at $r=0$. By transforming to isotropic coordinates $r=r^{\prime}+G M$ we can put the horizon of 1.77 at $r=0$ as well. The result reads

$$
\mathrm{d} s_{4}^{2}=-\frac{r^{\prime 2}}{\left(r^{\prime}+G M\right)^{2}} \mathrm{~d} t^{2}+\frac{\left(r^{\prime}+G M\right)^{2}}{r^{\prime 2}} \mathrm{~d} r^{\prime 2}+\left(r^{\prime}+G M\right)^{2} \mathrm{~d} \Omega_{2}^{2} .
$$

Taking the near-horizon limit $r \rightarrow 0$, we find the metric of $\mathrm{AdS}_{2} \times \mathrm{S}^{2}$. It turns out that for $Q_{1}=Q_{5}=Q_{n}$, the solution 1.70 is the five-dimensional cousin of the extremal Reissner-Nordström black hole, namely the Tangherlini black hole. More generally asymptotically flat supersymmetric black holes are always extremal and have thus vanishing temperature. Furthermore, the black holes we will be interested in always have an AdS factor in the near-horizon geometry. Note that supersymmetric black holes are not the ones we find in nature since those are never extremal. However, a counting of microstates of supersymmetric black holes is still an important test for string theory and the hope is that we will better understand how to count for the non-supersymmetric physical black holes.

For more general settings there is no explicit 10d or $11 \mathrm{~d}$ solution known like (1.67) because of the lack of explicit Calabi-Yau metrics. However, one does know the lower dimensional supergravity theory as we have seen in section 1.2 .3 .2 for type II theory on a Calabi-Yau threefold. Therefore it is possible to construct lower dimensional solutions corresponding to the settings of interest by finding black hole or black string solutions with the right properties, e.g. charges and amount of preserved supersymmetry. This is what we will do in chapter 2 for $6 \mathrm{~d}$ $\mathcal{N}=(1,0)$ supergravity which is the lower dimensional effective theory for F-theory on a Calabi-Yau threefold.

For computing corrections to the leading Bekenstein-Hawking area law one needs to determine (the generalization of) the Wald entropy 1.10. To get the full answer one in principle needs all higher derivative terms in the relevant action. However, in section 1.4.1 we will argue that we actually only need to know very specific terms in the action.

\subsubsection{Counting microstates}

We have seen how black holes in string theory correspond to certain brane constructions. The microstates of the black holes are captured by the worldvolume theory living on these branes. We would like to count them using perturbative open 
string theory which is only valid for $g_{s} N \ll 1$, where $N$ corresponds to the number of branes. However, the supergravity description of the branes is only valid for $g_{s} N \gg 1$ and $g_{s} \ll 1$. We can switch between the two parameter regimes by tuning $g_{s}$. We would thus count the microstates in another regime of the theory than where the macroscopic solution lives and it is not a priori clear why the answers would agree. We ignore this question for now and return to it in section 1.4.1. In the settings we consider the branes are wrapped on a geometry that contains a circle, such that we can consider the system in the limit where the radius $R$ of the circle is large compared to the characteristic length of the rest of the compact space. The worldvolume theory then becomes effectively two-dimensional. In the low energy limit, this theory flows to a $2 \mathrm{~d}$ superconformal field theory (SCFT) and it is in this theory that we can count the microstates. We will work in Euclidean signature where the time direction is periodic, i.e. $t_{\mathrm{E}} \sim t_{\mathrm{E}}+\beta$, with the periodicity corresponding to the inverse temperature $\beta=1 / T$ such that the SCFT is defined on a torus with modular parameter $\tau=i \frac{\beta}{2 \pi R}$. The SCFTs we are interested in for the counting of black hole microstates have at least $\mathcal{N}=(0,4)$ supersymmetry ${ }^{16}$ and are characterized by their central charges $c_{L, R}$ which show up in the Virasoro algebra. Furthermore, there is always a right-moving $S U(2)_{R}$ R-symmetry current with corresponding level $k_{R}$ related by supersymmetry to the right central charge as $c_{R}=6 k_{R}$. The Hamiltonian is equal to the sum of the Virasoro generators

$$
R H=L_{0}+\bar{L}_{0}-\frac{c_{L}+c_{R}}{24}
$$

and the momentum along the circle is given by the difference

$$
n=L_{0}-\bar{L}_{0}-\frac{c_{L}-c_{R}}{24} .
$$

We now sketch how the counting of microstates works, the finer details depend on the particular SCFT. Since the black holes we consider preserve four supercharges we want to count the states in the SCFT with the same amount of supersymmetry. One can show that these states obey 17

$$
\left(\bar{L}_{0}-\frac{c_{R}}{24}\right)|\mathrm{BPS}\rangle=0 .
$$

The microstates are captured by the partition function

$$
Z=\operatorname{tr} e^{-\beta H} .
$$

\footnotetext{
${ }^{16}$ The D1-D5 SCFT has $\mathcal{N}=(4,4)$ supersymmetry.

${ }^{17}$ Here we assumed supersymmetry in the right sector. If there is supersymmetry in both sectors of the SCFT as for the D1-D5 system this condition can be chosen without loss of generality. Also when the SCFT has more than just the small $\mathcal{N}=4$ superconformal algebra, the right side is not necessarily vanishing, but one can easily adapt the computation.
} 
Using the expressions of the Hamiltonian and momentum $n$ in terms of the Virasoro generators, this becomes

$$
Z(\tau)=\sum_{n=-\frac{c_{L}}{24}}^{\infty} d(n) e^{2 \pi i \tau n}
$$

where $d(n)$ corresponds to the degeneracy of states with momentum $n$. Rewriting the partition function as

$$
Z(q)=\sum_{n=-\frac{c_{L}}{24}}^{\infty} d(n) q^{n},
$$

where $q=e^{2 \pi i \tau}$, we can extract the degeneracy via

$$
d(n)=\frac{1}{2 \pi i} \oint_{C} \mathrm{~d} q Z(q) q^{-n-1},
$$

where the integral is over the unit circle in anticlockwise direction. 18 Reparametrizing to $\tau$ we find the integral

$$
d(n)=\frac{1}{2 \pi} \int \mathrm{d} \tau Z(\tau) e^{-2 \pi i \tau n},
$$

with a contour where $\operatorname{Im} \tau$ is very small. We then use that 1.83 is modular invariant and is dominated by the $n=-\frac{c_{L}}{24}$ term in the limit of $\operatorname{Im} \tau \rightarrow \infty$ such that we can approximate

$$
Z(\tau)=Z\left(-\frac{1}{\tau}\right) \approx d(0) e^{2 \pi i \frac{c_{L}}{24 \tau}}
$$

Plugging this into 1.86 we obtain

$$
d(n)=\frac{1}{2 \pi} \int \mathrm{d} \tau d(0) e^{2 \pi i\left(\frac{c_{L}}{24 n} \frac{1}{\tau}-\tau\right) n} .
$$

This integral can be computed in the Cardy limit $n \rightarrow \infty$ and $c_{L}$ finite using a saddle-point approximation in

$$
\tau_{0}=i \sqrt{\frac{c_{L}}{24 n}},
$$

which indeed has $\operatorname{Im} \tau_{0} \ll 1$. The saddle-point approximation results in

$$
d(n)=A e^{2 \pi \sqrt{\frac{c L}{6} n}}
$$

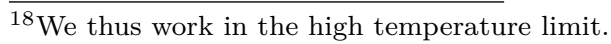


such that the entropy is given by

$$
S=2 \pi \sqrt{\frac{c_{L}}{6} n},
$$

up to subleading terms. This formula is known as the Cardy entropy and was first derived in [42]. Computing the entropy thus comes down to computing the left central charge of the SCFT. For the D1-D5 system this results in 38.

$$
c_{L}=6 N_{1} N_{5}+6
$$

which leads to an entropy

$$
S=2 \pi \sqrt{\left(N_{1} N_{5}+1\right) n}
$$

This reproduces the macroscopic number 1.76 in the limit of large charges. As already stated in the beginning of this section, we will explain in section 1.4.1 why the microscopic and macroscopic results match.

When there are additional symmetries in the SCFT, Cardy's formula 1.91 gets adapted by shifting the momentum $n$. In this thesis we will be interested in the case of a left-moving $S U(2)$ (or broken to $U(1)$ ) current algebra for which the Cardy formula becomes

$$
S=2 \pi \sqrt{\frac{c_{L}}{6}\left(n-\frac{J_{L}^{2}}{k_{L}}\right)},
$$

where $J_{L}$ is the corresponding eigenvalue and $k_{L}$ the corresponding level. This formula is valid in the limit $n-\frac{J_{L}^{2}}{k_{L}} \rightarrow \infty$. The entropy is still determined in terms of $c_{L}, k_{L}$ characterizing the SCFT and the counting of microstates comes down to computing these numbers. To do that we often depend on computations in the UV-theory, i.e. the compactified worldvolume theory, because generically it is not known what the IR CFT is. This theory has the same supersymmetry but is not necessarily conformal. One strategy to compute the central charges is by determining the number of (left- and right-moving) bosonic and fermionic degrees of freedom. Each boson adds 1 to the central charge whereas a fermion adds $\frac{1}{2}$. Another strategy uses that in the SCFT the levels $k_{L, R}$ and $c_{L}-c_{R}$ are related to anomaly coefficients, the levels to anomalies of the corresponding symmetries and the difference of central charges to the gravitational anomaly. These coefficients are the same in the UV and in the IR by 't Hooft anomaly matching and can therefore be computed without knowledge of the explicit IR SCFT. By using the value of $c_{L}-c_{R}$ and the relation $c_{R}=6 k_{R}$ both central charges can be computed. 
A huge number of works have been devoted to match microscopic and macroscopic entropy calculations. Apart from the D1-D5 black hole arising in the compactification of type IIB string theory on $\mathrm{T}^{4}, 38$ also discussed the same setting with $\mathrm{T}^{4}$ replaced by the Calabi-Yau manifold K3 that we encountered in section 1.2.3.2. Both these compactifications result in five-dimensional non-spinning black holes. Other prominent examples are the generalization of the D1-D5 system to include spin 43], five-dimensional non-spinning black holes in compactifications of M-theory on a Calabi-Yau threefold $\mathrm{CY}_{3}$ [4], and four-dimensional black holes in M-theory on $\mathrm{CY}_{3} \times \mathrm{S}^{1}$ 45. On the type IIB side four-dimensional extremal black holes may be obtained by adding a Kaluza-Klein monopole to the D-brane system [46, 47]. Furthermore, near-extremal black holes may be considered, see e.g. [39, 48].

\subsection{The AdS/CFT correspondence}

The matching of microscopic and macroscopic entropy can be seen as a test of the AdS/CFT correspondence 49 51]. This correspondence states that string theory in a $D$-dimensional AdS background is dual to a $(D-1)$-dimensional CFT. It is thus a correspondence between a theory including gravity and a theory without gravity. Apart from that it gives another perspective on the microstate counting we will also construct new examples of this correspondence in chapter 6

Let us illustrate the correspondence with a construction from the original paper [49]. Consider a flat type IIB background with a stack of $N$ D3-branes. As we have seen there are two ways of describing these branes: on the one hand using the microscopic open string description and on the other hand using a 3-brane supergravity solution 1.63. Interactions of the branes with closed strings can thus be studied either via the interaction of open string modes with closed string modes or by using type IIB string theory in the 3-brane background. The open string theory picture has a low-energy effective action which schematically consists of three parts, one to describe the worldvolume theory of the brane, one that describes the theory in the bulk and one that describes interactions between the two parts. In the limit that $\alpha^{\prime} \rightarrow 0$, while keeping other quantities such as $g_{s}$ and $N$ fixed, all massive string excitations decouple, and furthermore open and closed string modes decouple. We thus find that in this limit the whole system is described by two decoupled systems, i.e. the worldvolume theory of the branes and free type IIB supergravity in the bulk. The worldvolume theory is $\mathcal{N}=4$ supersymmetric Yang-Mills (SYM) theory and is conformal. When we use the other description of D3-branes, the 3-brane solution as string theory background, we have a similar 
decoupling limit. Namely at low energies, excitations in the region $r \ll L=Q_{3}^{1 / 4}$ decouple from excitations in the region $r \gg L$. In the latter region, which is flat, we have a free type IIB supergravity theory. It is thus equal to the bulk part of the open string description. Therefore it seems that we should identify the other parts in both descriptions, namely $\mathcal{N}=4 \mathrm{SYM}$ theory with gauge group $S U(N){ }^{19}$ and coupling $g_{\mathrm{YM}}^{2}=2 \pi g_{s}$ should be equal to type IIB string theory in the region $r \ll L$. The latter region is equal to $\mathrm{AdS}_{5} \times \mathrm{S}^{5}$ and because the five-sphere is compact we can consider this a five-dimensional theory. The precise dual string theory background is obtained by taking the near-horizon limit for the supergravity solution in the following way:

$$
\alpha^{\prime} \rightarrow 0, \quad r \rightarrow 0, \quad \frac{r}{\alpha^{\prime}} \text { fixed. }
$$

Let us discuss in which regime of the parameters this duality is valid. Note that on both sides we have assumed low energies. On the supergravity side we have neglected string loop effects by assuming $g_{s} \ll 1$ and we need that $L \gg \sqrt{\alpha^{\prime}}$ Otherwise higher derivative corrections in the action become important. Define the 't Hooft coupling $\lambda \equiv g_{\mathrm{YM}}^{2} N=2 \pi g_{s} N$ and recall 1.64 such that we get the relations

$$
g_{s}=\frac{\lambda}{2 \pi N}, \quad \frac{L^{2}}{\alpha^{\prime}}=\sqrt{2 \lambda}
$$

between the string and gauge theory parameters. To obtain the supergravity approximation to string theory, first take $N \rightarrow \infty$ and keep $\lambda$ fixed. In this limit $\lambda$ becomes the effective coupling constant. Subsequently take $\lambda \rightarrow \infty$. Therefore, the weakest form of the AdS/CFT correspondence states that $\mathcal{N}=4$ SYM theory with $S U(N)$ gauge group at large 't Hooft coupling $\lambda$ and in the limit $N \rightarrow \infty$ is equivalent to classical type IIB supergravity compactified on $\operatorname{AdS}_{5} \times \mathrm{S}^{5}$ with radius $L^{4}=2 \lambda \alpha^{\prime 2}$ and $N$ units of $F_{5}$ flux. The weakly coupled supergravity theory is thus dual to a strongly coupled quantum field theory. In a stronger form of the correspondence the theories are conjectured to be dual for arbitrary values of $g_{\mathrm{YM}}$ and $N$. The boundary of $\mathrm{AdS}_{5}{ }^{20}$ in Poincaré coordinates is $4 \mathrm{~d}$ Minkowski space on which the isometries of $\mathrm{AdS}_{5}$ act as conformal transformations. Therefore the field theory is said to be living on this boundary.

If the theories are dual there should be a dictionary between the two. For instance symmetries on both sides have to match. The isometry group of $\mathrm{AdS}_{5} \times \mathrm{S}^{5}$

\footnotetext{
${ }^{19}$ This is not $U(N)$ because there is a $U(1)$ factor associated to the center-of-mass motion of the branes that decouples.

${ }^{20}$ This is not a boundary in topological sense but a conformal boundary.
} 
is $S O(4,2) \times S O(6)$. This is indeed the maximal bosonic subgroup of the superconformal group the gauge theory is invariant under. Here $S O(4,2)$ is the conformal group in four dimensions while $S O(6)$ is the R-symmetry group of the theory. Recall that a D3-brane breaks the Lorentz group

$$
S O(1,9) \rightarrow S O(1,3) \times S O(6)
$$

to Lorentz transformations along the brane and the rotation group of the transverse space. The R-symmetry group can thus be identified with this rotation group.

We also need to know how string excitations on $\mathrm{AdS}_{5} \times \mathrm{S}^{5}$ map to operators in the CFT. In the supergravity limit we have the $5 \mathrm{~d}$ supergravity fields $\Phi_{i}$ with action resulting from the compactification of type IIB on $\mathrm{S}^{5}$. We can solve the equations of motion for $\Phi_{i}$ where we fix the boundary values to $\phi_{i}$. This results in an on-shell action depending on these boundary values. These $\phi_{i}$ can be interpreted as sources for operators $\mathcal{O}_{i}$ in the CFT, i.e. we get a one-to-one correspondence between fields in the bulk and operators in the CFT $\Phi_{i} \longleftrightarrow \mathcal{O}_{i}$, which is known as the state-operator correspondence. The string theory partition function is equal to the CFT partition function according to the duality. This equality reduces in the supergravity limit to

$$
\left.e^{-\mathrm{S}_{\text {sugra }}\left(\Phi_{i}\right)}\right|_{\delta S_{\text {sugra }}=0,\left.\Phi_{i}\right|_{\text {bdy }}=\phi_{i}}=Z_{\mathrm{CFT}}\left(\phi_{i}\right)=\left\langle\exp \left(\int \mathrm{d}^{4} x \phi_{i} \mathcal{O}_{i}\right)\right\rangle_{\mathrm{CFT}},
$$

where in the CFT we have to take the limit corresponding to the supergravity limit, i.e. $N \rightarrow \infty, \lambda \rightarrow \infty$ in the example we discussed. Once we know what the identification between fields and operators is, the identification 1.98 can be used to derive properties of the CFT from the gravity dual or vice versa

Since the advent of the AdS/CFT correspondence, many examples have been proposed. In particular one can replace the five-sphere by a Sasaki-Einstein manifold $Y_{5}$. This is a compact manifold whose cone,

$$
\mathrm{d} s^{2}=\mathrm{d} r^{2}+r^{2} \mathrm{~d} s_{Y_{5}}^{2},
$$

is Ricci-flat and Kähler. Even though the cone is not compact we will by slight abuse of language refer to it as Calabi-Yau. Note that the five-sphere is an example of a Sasaki-Einstein manifold where the corresponding cone is given by flat space. So following the same strategy as before one can construct other examples of the AdS/CFT correspondence. We thus consider a stack of D3-branes probing the cone of an arbitrary Sasaki-Einstein manifold. This is depicted in figure 1.5 The CFT side of the proposed correspondence is given by the worldvolume theory of 
the D3-branes which is a $4 \mathrm{~d} \mathcal{N}=1$ quiver gauge theory, while the gravity side is given by string theory on $\mathrm{AdS}_{5} \times Y_{5}$ which is the near-horizon geometry of the branes. A problem with obtaining evidence for these new correspondences is that the metric on $Y_{5}$ for generic Sasaki-Einstein manifolds is unknown. There are a few exceptions to this rule. These are given by the manifolds $Y_{5}=\mathrm{S}^{5}, T^{1,1}$, the family $Y_{5}=Y^{p, q}\left[52\right.$ characterized by integers $p$ and $q$, and the family $Y_{5}=L^{a, b, c} 53$ characterized by integers $a, b$ and $c 21$

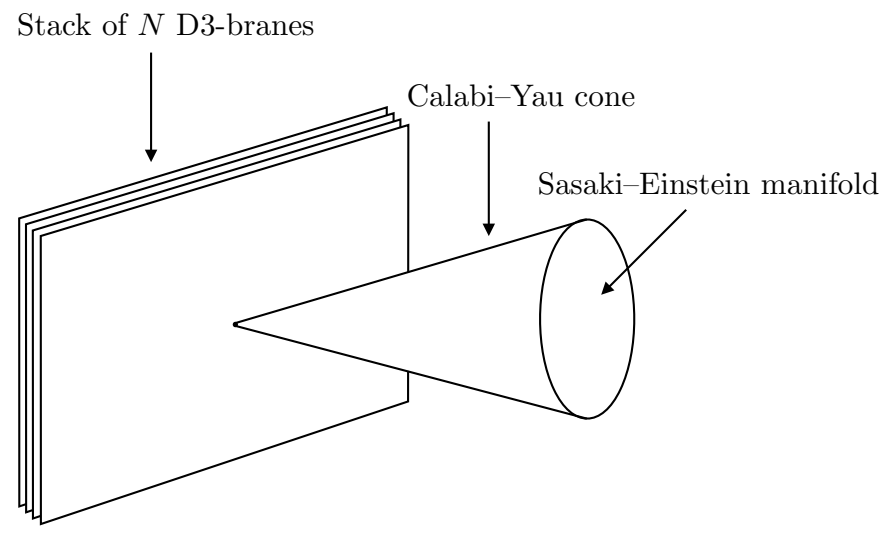

Figure 1.5: The construction of $\mathrm{AdS}_{5} / \mathrm{CFT}_{4}$ dualities via a stack of $N$ D3-branes probing the cone corresponding to a Sasaki-Einstein manifold $Y_{5}$. The field theory side is given by the worldvolume theory of the branes and the gravity side is equal to string theory on $\mathrm{AdS}_{5} \times Y_{5}$ which is the near-horizon geometry of the branes.

\subsubsection{Microstate counting and the AdS/CFT correspondence}

In this thesis we will be mostly interested in lower dimensional examples of the AdS/CFT correspondence, namely in dualities between $\mathrm{AdS}_{3}$ and $2 \mathrm{~d}$ CFTs. We have already seen how to construct some of these examples when we discussed black holes in section 1.3 They had uplifts to black strings arising from branes wrapping curves in a compact manifold. String theory on the near-horizon geometry of these black strings is the geometry side of the dualities while the IR fixed point of the compactified worldvolume theory of the branes is the field theory side. The

\footnotetext{
${ }^{21}$ There is thus also a metric for the cone of these manifolds which is Calabi-Yau. These cones are non-compact in contrast to the compact Calabi-Yau manifolds we examined in section 1.2.3.2 for which (apart from the torus) no explicit metrics are known.
} 
computation of black hole entropy at both sides may be seen as a test of the dualities. We have seen that the quantities of interest for microstate counting are the central charges $c_{L, R}$ and levels $k_{L, R}$ of the CFT. According to the correspondence it should be possible to derive these from the geometry side. As we will see this enables us to obtain the entropy of the black holes without knowledge of the full action which would in principle be necessary when using the Wald formalism (1.10). Let us first discuss how one finds the central charges and levels from $\mathrm{AdS}_{3}$ and after we examine how these can be used to compute the entropy.

We can write the $\mathrm{AdS}_{3}$ metric in global coordinates as

$$
\mathrm{d} s^{2}=-\left(1+\frac{r^{2}}{L^{2}}\right) \mathrm{d} t^{2}+\frac{\mathrm{d} r^{2}}{1+\frac{r^{2}}{L^{2}}}+r^{2} \mathrm{~d} \phi^{2},
$$

where $-\infty<t<\infty, 0 \leq r<\infty$ and $0 \leq \phi<2 \pi$. Assuming $c_{L}=c_{R}$ in the dual $\mathrm{CFT}^{22}$ the central charges are given by 54,55

$$
c_{L, R}=\frac{L}{2 G^{(3)}} g_{\mu \nu} \frac{\partial \mathcal{L}}{\partial R_{\mu \nu}}
$$

where $\mathcal{L}$ is the Lagrangian density and $G^{(3)}$ is the three-dimensional gravitational constant. In the limit that the action is given by two-derivative gravity we thus find that

$$
c_{L, R}=\frac{3 L}{2 G^{(3)}},
$$

which is the celebrated Brown-Henneaux formula 56.

The levels in the CFT are related to anomaly coefficients of the corresponding symmetries. This is the reason why they are determined by the coefficients of the corresponding Chern-Simons terms in the $3 \mathrm{~d}$ action. The precise relation is (see e.g. [57])

$$
\frac{k_{L}}{8 \pi} \int A_{L} \wedge F_{L}+\frac{k_{R}}{4 \pi} \int \operatorname{tr}\left(A_{\mathrm{R}} \wedge \mathrm{d} A_{R}+\frac{2}{3} A_{R}^{3}\right) \subset S_{3}
$$

where $k_{L, R}$ are positive and integer. Furthermore, $A_{L}$ is a $U(1)$ gauge field corresponding to a left-moving current in the CFT and $A_{R}=-A_{R}^{a} \frac{i}{2} \sigma^{a}$ an $S U(2)$ gauge field in terms of the Pauli matrices corresponding to a right-moving current. We have picked these particular gauge fields because they are the most relevant for this thesis. Generically $U(1)$ gauge fields corresponding to left-moving (right-moving) currents come with a positive (negative) sign in the action and $S U(2)$ gauge fields corresponding to right-moving (left-moving) currents come with a positive (negative)

${ }^{22}$ As we will see this is the case if the three-dimensional action does not contain the gravitational Chern-Simons term. 
sign. Chern-Simons terms corresponding to general non-abelian groups will be important in section 4.5 and follow the same normalizations as $S U(2)$ Chern-Simons terms.

As we discussed before, when we have at least $\mathcal{N}=(0,4)$ supersymmetry in the SCFT the right central charge $c_{R}$ is related to the level of the $S U(2)_{R} \mathrm{R}$ symmetry current as $c_{R}=6 k_{R}$. We can then determine $c_{R}$ by only knowing the $S U(2)_{R}$ Chern-Simons term in the 3d action. Compare this with 1.101 for which knowledge of the full action is required. However, this does not give us $c_{L}$ which we need in the Cardy formula. Fortunately, $c_{L}-c_{R}$ corresponds to the gravitational anomaly. Therefore, its value is governed by the coefficient of the $3 \mathrm{~d}$ gravitational Chern-Simons term: 55,58

$$
\frac{c_{L}-c_{R}}{96 \pi} \int \operatorname{tr}\left(\omega \wedge \mathrm{d} \omega+\frac{2}{3} \omega^{3}\right) \subset S_{3},
$$

where $\omega$ is the spin connection. Thus $c_{L, R}$ and $k_{L, R}$ can be computed in $\mathrm{AdS}_{3}$ by determining the Chern-Simons terms 1.103 and 1.104.

This then provides us with a strategy for determining the black hole entropy from macroscopics. The supersymmetric black holes we consider in this thesis can always be uplifted to a higher dimensional black string and thus have a near-horizon geometry with an $\mathrm{AdS}_{3}$ factor. This black string solves the equations of motions of a certain effective action. We can then reduce this effective action in the near-horizon geometry of the black string in order to obtain an effective 3d action. Subsequently we can read off the levels and difference of the central charges from the coefficients of the Chern-Simons terms 1.103 and 1.104 . The entropy should then be given by Cardy's formula. In [54,55] it was shown that the entropy determined this way equals the Wald entropy (1.10). We would like to stress again that for computing the entropy using the Wald formula one in principle has to know all higher derivative terms in the effective action. For the method using Chern-Simons terms one just has to know the terms in the higher dimensional action that can possibly lead to lower dimensional Chern-Simons terms. These are generally finite in number.

We can now finally argue why the microscopic and macroscopic entropies match even though they are computed in different parameter regimes of the theory. The reason is of course the AdS/CFT correspondence. However, as explained in 55] one can alternatively use an argument based on anomaly inflow [59]. We can switch between the perturbative open string description of D-branes and the supergravity description by tuning $g_{s}$. For small $g_{s} N$ in the low-energy limit we have the worldvolume theory coupled to the bulk. This theory has a symmetry group which can be associated to the rotation group of the transverse space. For instance, when 
the transverse space is $\mathbb{R}^{4}$ we have the symmetry group $S O(4)=S U(2)_{L} \times S U(2)_{R} 2^{23}$ This symmetry is generally anomalous with anomalies determined by the levels $k_{L, R}$. However, from the $10 \mathrm{~d}$ viewpoint this cannot happen, the rotation group corresponds to coordinate transformations and it is known that the full theory is non-anomalous. Therefore, the bulk theory needs to have an $S O(4)$ variation of the action canceling that of the worldvolume theory. In other words, there is an anomaly inflow from the bulk onto the brane. We can then increase the string coupling $g_{s}$ to get to the supergravity description of D-branes where we have the near-horizon AdS geometry joined to an asymptotically flat region. Since the inflow from the bulk is determined in terms of the integers $k_{L, R}$ it does not change when increasing $g_{s}$. In the supergravity picture current flows smoothly across the border of the two regions which is why the AdS current inflow matches the one from the asymptotic region. Therefore, the AdS current inflow should match the brane anomaly and since both are determined by the parameters $k_{L, R}$ these numbers should match. One can use the same argument for $c_{L}-c_{R}$ since it determines the gravitational anomaly in the CFT. If we associate, both at the microscopic and macroscopic sides, the $S U(2)_{R}$ symmetry with the R-symmetry of the SCFT we can use the relation $c_{R}=6 k_{R}$, determine $c_{L}$ and compute the Cardy entropy. This then justifies why the microscopic and macroscopic entropies match.

Computing an index instead of the degeneracy. As pointed out in 60 there is a subtlety with the justification provided above. The argument for the matching of the microscopic and macroscopic left central charge relied on the identification of the $S U(2)$ R-symmetry group with part of the symmetry group of the transverse space. Microscopically this identification is indeed correct for the part of the worldvolume theory that controls most of the entropy, however it can also fail for part of the spectrum. An example is provided by the modes representing transverse oscillations of the branes. We will show this in more detail in chapter 5 . Generically it might thus be hard to find an exact matching of microscopic and macroscopic degeneracies if one also includes corrections to the leading order contribution. Therefore, it is more interesting to compute an object that inherently does not depend on $g_{s}$. Indeed instead of computing the partition function 1.82 one can compute an object of the form

$$
\operatorname{tr}(-1)^{F} e^{-\beta H}
$$

${ }^{23}$ For the settings we consider in this thesis the symmetry group will be either $U(1)_{L} \times S U(2)_{R}$ or just $S U(2)_{R}$. 
where $F$ is the Fermion number. The particular object 1.105 is the Witten index 61 and counts the groundstates of an $\mathcal{N}=1$ theory with a positive sign for bosons and a negative sign for fermions. In a supersymmetric theory the massive states come in pairs and their contributions thus cancel out. This object is protected against continuous changes of the parameters that preserve the supersymmetry The trace 1.105 might vanish for the particular theory at hand ${ }^{24}$ but can be adapted by replacing $(-1)^{F} \rightarrow(-1)^{F} g(F)$, where $g$ is a certain function of $F$, in order to account for the microstates in a similar fashion 62, 63]. For a number of brane constructions it has been shown that in the Cardy limit the leading order contribution to the relevant index is of the form 1.94. However now with $c_{L}$ computed from $c_{L}-c_{R}$ and $c_{R}=6 k_{R}$ in addition to $k_{L, R}$ and $c_{L}-c_{R}$ being obtained from respectively the anomalies of the symmetry group of the transverse space and the gravitational anomaly $\left[60\right.$. Generically this $c_{L}$ is thus not the left central charge, but does agree with it to leading order. Therefore, in general the index agrees with the microscopic degeneracy to leading order.

On the macroscopic side one can also define an index. Following an argument in 64] we show that also this object agrees with the degeneracy to leading order. The macroscopic index can be written as the sum of products of the contributions from the horizon and the hair which are degrees of freedom living outside of the horizon [64 66. The part corresponding to the horizon comes with a weight factor $\exp (2 \pi i J)$ where $J$ is the third component of angular momentum ${ }^{25}$ The near-horizon geometry of the black hole has an $\mathrm{AdS}_{2}$ factor and the natural boundary condition on fields in $\mathrm{AdS}_{2}$ is to fix their charges, in particular the angular momentum, and let the dual potentials fluctuate 67,68 . So a spherically symmetric horizon will represent an ensemble of states with zero angular momentum. All states associated to the horizon thus have $\exp (2 \pi i J)=1$, which implies that for these states the index is the same as the degeneracy. Therefore for these states which form the leading order contribution to the degeneracy, the index is equal to the entropy. The contribution of the hair modes to the index might be different from the degeneracy. As shown in 60] the leading contribution (including the hair modes) to the macroscopic index can be computed with a Cardy formula where the central charges and levels are again computed from $k_{L, R}$ and $c_{L}-c_{R}$. However, the latter data is not computed from coefficients of Chern-Simons terms in the effective $3 \mathrm{~d}$ action that one gets by reducing the higher dimensional action in the near-horizon geometry of the black string. Instead they correspond to the coefficients of Chern-Simons terms

\footnotetext{
${ }^{24}$ This depends on the specific algebra of the SCFT.

${ }^{25}$ Recall that for the black holes we consider there always is an $S U(2)_{R}$ symmetry group.
} 
(1.103 and (1.104) that one gets by reducing the higher dimensional action in the asymptotic geometry of the black string. Since these coefficients correspond to the anomalies on the brane configuration the microscopic and macroscopic index agree 60.

In chapters 3.5 we compute the anomalies of the rotation group of the transverse space $k_{L, R}$ and the gravitational anomaly $c_{L}-c_{R}$ in various settings. Using the relation $c_{R}=6 k_{R}$ and the value of $c_{L}-c_{R}$ to determine $c_{L}$ results in the coefficient that together with $k_{L}$ generally determines the leading order contribution to the index 26 and not the degeneracy, even though the two objects agree to leading order in the charges. We will keep referring to $c_{L, R}$ determined this way as central charges.

\subsubsection{New 3D/2D examples of the AdS/CFT correspondence}

We have seen how black strings yield examples of the correspondence between a $2 \mathrm{~d}$ SCFT and string theory on an effectively $\mathrm{AdS}_{3}$ geometry. The black strings we considered so far arose from branes wrapping curves in a compact manifold. However, we can also construct examples in a slightly different way. Recall that we can construct $\mathrm{AdS}_{5} / \mathrm{CFT}_{4}$ examples by considering D3-branes probing the cone of a Sasaki-Einstein space. When we additionally wrap the brane on a Riemann surface we can construct $\mathrm{AdS}_{3} / \mathrm{CFT}_{2}$ examples. The gauge theory side is the IR fixed point of the four-dimensional gauge theory compactified on the Riemann surface. This results in a $2 \mathrm{~d}$ SCFT with $\mathcal{N}=(0,2)$ supersymmetry. The geometry side should be string theory on the near-horizon geometry of the wrapped D3-brane. This geometry is a product consisting of an $\mathrm{AdS}_{3}$ factor and a factor in which the Sasaki-Einstein manifold is fibered over the Riemann surface. Preserving supersymmetry puts strong restrictions on the geometry and it turns out that with a well-chosen ansatz one can find new $\mathrm{AdS}_{3} / \mathrm{CFT}_{2}$ examples. This has been successfully applied to the cases where the Sasaki-Einstein manifold is given by $\mathrm{S}^{5} 69 \sqrt{73}$ and $Y^{p, q}$ [74, and in chapter 6 we extend this to $Y_{5}=L^{a, b, c}$. The most important part will be to construct the new $\mathrm{AdS}_{3}$ solutions. We will obtain evidence for the new dualities by computing data such as the central charge 1.102 from both sides and show that they match. These solutions do have a black string interpretation as well. They can be thought of as the near-horizon geometries of black strings in an asymptotically $\mathrm{AdS}_{5} \times Y_{5}$ space. Computing the central charge of the dual CFT is related to computing the entropy density of these strings.

${ }^{26}$ We will not show this for the settings we consider. 


\subsection{Outline}

In this section we give an overview of the work presented in this thesis. The largest part, namely chapters 3.5 , will be about computing central charges and levels corresponding to supersymmetric black strings arising in compactifications of F-theory on an elliptically fibered Calabi-Yau threefold $\mathrm{CY}_{3}$ or of type IIB string theory on K3. As we discussed in section 1.2.5, the F-theory compactifications result in effective six-dimensional $\mathcal{N}=(1,0)$ supergravity theories. The black strings we are interested in are supersymmetric solutions of these theories. Since we always set the vectors in the vector multiplets to zero and the hyperscalars in the hypermultiplets to constants, they solve $6 \mathrm{~d} \mathcal{N}=(1,0)$ supergravity coupled to tensor multiplets. This theory is also realized as a truncation of the $\mathcal{N}=(2,0)$ theory one gets when compactifying type IIB supergravity on K3. Our first step in chapter 2, which is based on [1], will be to examine supersymmetric solutions of $\mathcal{N}=(1,0)$ supergravity coupled to tensor multiplets. We derive a general local form for the supersymmetric solutions after which we consider some special cases in which the resulting equations can be solved explicitly. This way we also find black string solutions relevant for the later chapters.

In chapter 3, based on [2], we will in particular be interested in solutions corresponding to black strings in F-theory that have a Taub-NUT space $\mathrm{TN}_{m}$ transversely. The strings arise from D3-branes in an asymptotic F-theory geometry $\mathbb{R} \times \mathrm{TN}_{m} \times \mathrm{S}^{1} \times \mathrm{CY}_{3}$ where the D3-brane is wrapped on a curve in the base of the elliptically fibered Calabi-Yau threefold and on $\mathrm{S}^{1}$. The Taub-NUT space is a circle fibration over $\mathbb{R}^{3}$ which is why this setting corresponds to four-dimensional black holes. Recall from section 1.2.5 that the fiber of the threefold degenerates along the divisors in the base where 7-branes are located in the corresponding type IIB setting. These degenerations may or may not result in singularities of the whole threefold. In chapter 3 we only consider fibrations that are not singular. In the corresponding $6 \mathrm{~d}$ supergravity theory this corresponds to not having vector multiplets. Microscopically the black strings are described by a $2 \mathrm{~d} \mathcal{N}=(0,4)$ SCFT. The central charges and levels (related to the symmetries of the Taub-NUT space) of this SCFT have been computed from a microscopic point of view in 75 . In chapter 3 we reproduce them from macroscopics using the techniques outlined in section 1.4.1 We thus compactify the 6d supergravity theory on the spherical part of the black string solution and read off the central charges and levels from the $3 \mathrm{~d}$ Chern-Simons terms. Recall from section 1.2 .3 that for generic compactifications one cannot just ignore the massive Kaluza-Klein modes. Indeed for the settings we 
consider these modes contribute to the Chern-Simons terms via one-loop diagrams in which they run in the loop. A large part of chapter 3 will be about computing these quantum contributions.

In chapter 4, which is based on work that still has to appear [5], we generalize the setting we just discussed in two ways. Taub-NUT is an example of an asymptotically locally flat (ALF) space. Therefore, a first generalization is to consider D3-branes in asymptotic F-theory geometries $\mathbb{R} \times M_{4} \times \mathrm{S}^{1} \times \mathrm{CY}_{3}$ where $M_{4}$ is a general ALF space. In addition we consider asymptotically locally Euclidean (ALE) transverse spaces. A second generalization is to study the effect of elliptically fibered threefolds in which the degenerations along certain divisors do lead to singularities of the whole fibration. These fibrations yield supergravity theories with vector multiplets that correspond to gauge symmetries of the $6 \mathrm{~d}$ bulk theory. From the perspective of the string, this leads to non-abelian flavour symmetries of the $2 \mathrm{~d} \mathcal{N}=(0,4)$ SCFTs, which have associated levels. We compute the central charges and levels corresponding to these settings using their macroscopic $6 \mathrm{~d}$ description.

Apart from the cases in which the transverse space is given by Taub-NUT or by $\mathbb{R}^{4}$ (see e.g. 76]) the techniques are not available yet to reproduce the central charges and levels with a microscopic computation. This is the reason that in chapter 5, which is based on [3], we study black strings with an ALE or ALF space transversely in type IIB string theory without 7-branes. That is, we consider D3-branes in an asymptotic $\mathbb{R} \times M_{4} \times \mathrm{S}^{1} \times \mathrm{K} 3$ geometry where the D3-branes are wrapped on a curve in $\mathrm{K} 3$ and on $\mathrm{S}^{1}$. We compute the corresponding central charges and levels both from the macroscopic black string description and using a microscopic derivation. We find that the two computations agree.

Lastly, in chapter 6, which is based on [4], we change gears a little and consider the near-horizon geometries of asymptotically $\mathrm{AdS}_{5} \times L^{a, b, c}$ black strings in type IIB. In particular we construct solutions of the form $\operatorname{AdS}_{3} \times Y_{7}$ where $Y_{7}$ is a fibration of $L^{a, b, c}$ over a Riemann surface. With these new solutions we find new examples of the $\mathrm{AdS}_{3} / \mathrm{CFT}_{2}$ correspondence. We compute data such as the central charge on both sides of the correspondence and show that the two results match. 



\section{BPS solutions of 6D $(1,0)$ supergravity coupled to tensor multiplets}

In the first part of this thesis we are interested in the central charges and levels corresponding to certain black holes arising in compactifications of F-theory (chapters 3 and 4 ) and type IIB string theory (chapter 5). We will compute this data from a macroscopic viewpoint using the six-dimensional uplift of these black holes which is given by black strings. Finding explicit solutions describing these black strings will be one of the goals of this chapter. The specific theory we are interested in is six-dimensional $\mathcal{N}=(1,0)$ supergravity coupled to matter multiplets which is the result of compactifying F-theory on elliptically fibered Calabi-Yau threefolds or a truncation of type IIB on $\mathrm{T}^{4}$ or K3. Even though the black string solutions will be of most interest to us and be used in the subsequent three chapters, we will consider more general supersymmetric solutions of this theory. So far general BPS solutions of $\mathcal{N}=(1,0)$ supergravity have mainly been studied in the minimal case [77] and in the case with the gravity multiplet coupled to one or two tensor multiplets (gauged and ungauged), see e.g. 78 87. In this chapter, which is based on [1], we generalize this to $n_{T}$ tensor multiplets, for any $n_{T} \bigsqcup^{1}$ Even though the supergravity theories in chapters 3.5 generically do not fall in this class, we will always be able to truncate them such that the relevant black strings are solutions of the theory with just tensor multiplets.

\subsection{Introduction}

The phase space of (supersymmetric) gravity solutions in dimensions larger than four is intricate and has a rich structure, see e.g. 89,90 for some reviews on black holes and horizons in various dimensions. Whereas in asymptotically flat four spacetime dimensions, horizon topologies are unique to be $\mathrm{S}^{2}$, in five dimensions one can have

\footnotetext{
${ }^{1}$ See also 88 which was published at the same time as the paper 1 on which this chapter is based. The paper 88 considers supersymmetric solutions when the gravity multiplet is coupled to tensor multiplets, hypermultiplets and vector multiplets. However, explicit solutions are only minimally studied in this paper.
} 
black holes and black rings [91], with horizons $\mathrm{S}^{3}$ and $\mathrm{S}^{1} \times \mathrm{S}^{2}$ respectively. Also quotient topologies exist, such as Lens space horizons 92 94. In six dimensions, near-horizon geometries have been classified in $\mathcal{N}=(1,0)$ supergravity coupled to tensor multiplets and hypermultiplets (and no vector multiplets) [77,95], and there are more possibilities. The focus of this chapter, the case where the hypermultiplets are frozen, only yields near-horizon geometries locally given by either $\mathbb{R}^{1,1} \times \mathrm{T}^{4}$, $\mathbb{R}^{1,1} \times \mathrm{K} 3$ or $\mathrm{AdS}_{3} \times \mathrm{S}^{3}$. Some of the objects with a horizon easily follow from uplifts from $5 \mathrm{~d}$ to $6 \mathrm{~d}$. The most well studied case is of course the $6 \mathrm{~d}$ BPS black string with horizon $\mathrm{S}^{1} \times \mathrm{S}^{3}$ and near-horizon geometry $\mathrm{AdS}_{3} \times \mathrm{S}^{3}$, arising from the uplift of a $5 \mathrm{~d}$ spherical black hole. One can also uplift a $5 \mathrm{~d}$ black ring and we will see that it also has horizon $\mathrm{S}^{1} \times \mathrm{S}^{3}$ and near-horizon geometry $\mathrm{AdS}_{3} \times \mathrm{S}^{3}$. Besides black objects with horizons, there are smooth horizonless BPS (microstate-) geometries (see [80, 83 87. for an incomplete list of references), and an interesting class of $6 \mathrm{~d}$ BPS pp-wave solutions that we will study in this chapter. Many other solutions can be found by superposing waves on black strings, and one can play with various kinds of asymptotics.

To find a classification of supersymmetric solutions, our analysis starts with deriving a general local form for supersymmetric solutions where we use methods that have been applied before to four-dimensional theories [96 101], five-dimensional theories 102 105 and minimal six-dimensional supergravity 77 . The strategy is always the same. Starting from a Killing spinor $\epsilon$ one constructs bosonic objects quadratic in $\epsilon$, the so-called bilinears, such as the vector $X^{\mu}=\bar{\epsilon} \gamma^{\mu} \epsilon$. These bilinears have certain properties since $\epsilon$ is a Killing spinor. The vector $X^{\mu}$ for instance turns out to be a Killing vector in all cases. Using these bilinears, the local form of the solutions can be identified and this can be used to simplify the equations of motion. It turns out that the resulting equations in minimal $\mathcal{N}=24 \mathrm{~d}$ supergravity can actually be solved completely 97 . In five-dimensional minimal supergravity the solutions fall into two classes $[102$. In the first class, the vector $X$ is null and the solutions are plane-fronted waves expressed in harmonic functions on $\mathbb{R}^{3}$. In the second class, the vector $X$ is timelike and the equations of motion cannot be solved completely, but the equations are simplified significantly such that one only has to make an ansatz for the remaining variables. Solutions of supergravity coupled to an arbitrary number of vector multiplets have similar properties as in the minimal case [104]. In six-dimensional minimal supergravity the Killing vector $X$ is always null 77 and as one can expect, based on the five-dimensional analysis, the equations of motion cannot be solved completely in the most general case.

The bosonic field content in the six-dimensional theory consists of the metric, 
a two-form with anti-self-dual field strength in the gravity multiplet, and $n_{T}$ twoforms with self-dual field strength and $n_{T}$ scalars in the tensor multiplets. The constraints that supersymmetry puts on the field content have already been derived in 106, so like in [77] we will introduce coordinates and reduce the equations of motion using these local expressions. Using coordinates $\left(u, v, x^{m}\right), m=1, \ldots, 4$ we find that solutions are not dependent on $v$ and can be expressed in terms of a $u$-dependent base manifold $\mathcal{B}$. In general the base space $\mathcal{B}$ exhibits a non-integrable hyper-Kähler structure. Using the form of the solutions that one gets from requiring one Killing spinor, all the equations can be expressed in terms of bosonic quantities on $\mathcal{B}$. These equations are not easy to solve in full generality, but it is still easier to find solutions by substituting an ansatz in these equations than to start with an ansatz for the complete field content.

The resulting equations will be studied in two cases where the base space becomes hyper-Kähler. One of those cases arises when the solution is $u$-independent. When we additionally take $\mathcal{B}$ to be Gibbons-Hawking 107 it turns out that the solution is completely determined in terms of $6+2 n_{T}$ harmonic functions on $\mathbb{R}^{3}$. When one takes these solutions to be multi-centered Gibbons-Hawking, requiring the absence of Dirac string-like singularities gives restrictions on the relative positions of the centers. Just as in the minimal case 108 there is a symplectic group that sends solutions to solutions preserving regularity, but here the $S p(6)$ gets enlarged to $S p\left(6+2 n_{T}\right)$.

This chapter is organized as follows. In section 2.2 we describe the field content of $\mathcal{N}=(1,0)$ supergravity with a gravity multiplet and tensor multiplets, we list the conditions the existence of a Killing spinor puts on the field content, and we introduce the equations of motion that are not implied by integrability conditions. In section 2.3 we introduce coordinates and reduce the equations of motion to equations in terms of bosonic quantities on $\mathcal{B}$. Section 2.4 then solves the resulting equations under certain assumptions. Here we also discuss how the theory reduces to five dimensions and look at black strings and other objects with a horizon. We finish this section by looking at examples of pp-waves. In section 2.5 we then conclude.

Notation. Since we use a lot of different notation in this chapter, we give a short overview:

- $M, N=1, \ldots, n_{T}$ where $n_{T}$ is the number of tensor multiplets,

- $\alpha, \beta, \ldots=1, \ldots, n_{T}+1$ denote field content in six dimensions, 
- $\mu, \nu, \ldots=0, \ldots, 5$ denote coordinates or the vielbein of the six-dimensional metric,

- $i, j, k, l=1, \ldots, 4$ first denote part of the vielbein of the six-dimensional space and from section 2.3 onwards the vielbein of the base manifold $\mathcal{B}$,

- $m, n, \ldots=1, \ldots, 4$ denote the coordinates of the base manifold $\mathcal{B}$,

- $a, b, c, d=1, \ldots, 3$ denote either the three two-forms in the almost hyperKähler structure, or part of the vielbein of a Gibbons-Hawking metric,

- $I, J, \ldots=0, \ldots, n_{T}+1$ denote field content in five dimensions,

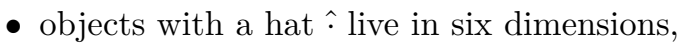

- objects with a tilde ? live in the four-dimensional base space,

- $\hat{*}$ is the Hodge star in six dimensions,

- $*_{4}$ is the Hodge star in the four-dimensional base space.

\subsection{Setting}

We consider six-dimensional $\mathcal{N}=(1,0)$ supergravity coupled to $n_{T}$ tensor multiplets 109 111. Vector and hypermultiplets can be added to ensure an F-theory embedding, but we will set them to zero in the solutions we consider in this chapter. The idea is to study the BPS structure of the tensor branch. The bosonic content of the gravity multiplet consists of a graviton and a two-form with anti-self-dual field strength. Every tensor multiplet contains a two-form with self-dual field strength and a scalar. We will denote the six-dimensional two-forms by $\hat{B}^{\alpha}$, where $\alpha=1, \ldots, n_{T}+1$. The scalars of the tensor multiplets parametrize the coset space $S O\left(1, n_{T}\right) / S O\left(n_{T}\right)$. A convenient way to describe them is by an $S O\left(1, n_{T}\right)$ matrix

$$
S=\left(\begin{array}{c}
\hat{\jmath}_{\alpha} \\
x_{\alpha}^{M}
\end{array}\right), \quad M=1, \ldots, n_{T},
$$

whose matrix elements satisfy the constraints

$$
\hat{\jmath}_{\alpha} \hat{\jmath}_{\beta}-\sum_{M} x_{\alpha}^{M} x_{\beta}^{M}=\Omega_{\alpha \beta}, \quad \hat{\jmath}_{\alpha} \hat{\jmath}^{\alpha}=1, \quad x_{\alpha}^{M} \hat{\jmath}^{\alpha}=0
$$

where $\Omega_{\alpha \beta}=\operatorname{diag}(1,-1, \ldots,-1)$ is used to lower and raise $\alpha$ indices. The field strengths corresponding to the two-forms are given by $\hat{G}^{\alpha}=\mathrm{d} \hat{B}^{\alpha}$ and their relation 
to the anti-self-dual tensor $H$ of the gravity multiplet and the self-dual tensors $H^{M}$ of the tensor multiplets is given by

$$
\hat{G}^{\alpha}=\hat{\jmath}^{\alpha} H-\Omega^{\alpha \beta} \sum_{M} x_{\beta}^{M} H^{M} .
$$

In terms of the three-forms $\hat{G}^{\alpha}$ the self-duality condition can be written as

$$
g_{\alpha \beta} \hat{*} \hat{G}^{\beta}=-\Omega_{\alpha \beta} \hat{G}^{\beta},
$$

where

$$
g_{\alpha \beta}=2 \hat{\jmath}_{\alpha} \hat{\jmath}_{\beta}-\Omega_{\alpha \beta}
$$

is a positive definite metric.

The self-duality condition on the three-forms makes it hard to construct a covariant action functional from which all equations of motion follow. These actions do exist 112,113, but one has to introduce auxiliary fields. Another approach is a pseudo-action from which equations of motion follow that then still have to be supplemented by the self-duality condition. The bosonic part of the pseudo-action that is relevant for this chapter is given by 111

$$
S=\int_{M_{6}}\left[\frac{1}{2} \hat{R} \hat{*} 1-\frac{1}{4} g_{\alpha \beta} \hat{G}^{\alpha} \wedge \hat{*} \hat{G}^{\beta}+\frac{1}{2} \Omega_{\alpha \beta} \mathrm{d} \hat{\jmath}^{\alpha} \wedge \hat{*} \mathrm{~d} \hat{\jmath}^{\beta}\right] .
$$

The equations of motion are then equal to

$$
\begin{aligned}
\hat{R}_{\mu \nu} & =\frac{1}{4} g_{\alpha \beta} \hat{G}_{\mu}^{\alpha \rho \lambda} \hat{G}_{\nu \rho \lambda}^{\beta}-\frac{1}{24} \hat{g}_{\mu \nu} g_{\alpha \beta} \hat{G}_{\rho \lambda \sigma}^{\alpha} \hat{G}^{\beta \rho \lambda \sigma}-\Omega_{\alpha \beta} \partial_{\mu} \hat{\jmath}^{\alpha} \partial_{\nu} \hat{\jmath}^{\beta}, \\
x_{\alpha}^{M} \mathrm{~d}\left(\hat{*} \mathrm{~d} \hat{\jmath}^{\alpha}\right) & =-x_{\alpha}^{M} \hat{\jmath}_{\beta} \hat{G}^{\alpha} \wedge \hat{*} \hat{G}^{\beta}, \\
\mathrm{d}\left(g_{\alpha \beta} \hat{*} \hat{G}^{\beta}\right) & =0 \\
g_{\alpha \beta} \hat{*} \hat{G}^{\beta} & =-\Omega_{\alpha \beta} \hat{G}^{\beta}, \\
\hat{\jmath}_{\alpha} \hat{\jmath}^{\alpha} & =1 .
\end{aligned}
$$

Using the self-duality condition, this set of equations is equivalent to

$$
\begin{aligned}
\hat{R}_{\mu \nu} & =\frac{1}{4} g_{\alpha \beta} \hat{G}_{\mu}^{\alpha}{ }^{\rho \lambda} \hat{G}_{\nu \rho \lambda}^{\beta}-\Omega_{\alpha \beta} \partial_{\mu} \hat{\jmath}^{\alpha} \partial_{\nu} \hat{\jmath}^{\beta}, \\
x_{\alpha}^{M} \mathrm{~d}\left(\hat{*} \mathrm{~d} \hat{\jmath}^{\alpha}\right) & =x_{\alpha}^{M} \hat{\jmath}_{\beta} \hat{G}^{\alpha} \wedge \hat{G}^{\beta}, \\
\mathrm{d} \hat{G}^{\alpha} & =0 \\
g_{\alpha \beta} \hat{*} \hat{G}^{\beta} & =-\Omega_{\alpha \beta} \hat{G}^{\beta}, \\
\hat{\jmath}_{\alpha} \hat{\jmath}^{\alpha} & =1 .
\end{aligned}
$$

Note that the equation of motion for the three-forms becomes the Bianchi identity. 


\subsection{1 $\mathcal{N}=1$ restrictions}

The BPS equations for six-dimensional $\mathcal{N}=(1,0)$ supergravity with a gravity multiplet and tensor multiplets are 106

$$
\begin{aligned}
\left(\nabla_{\mu}-\frac{1}{8} H_{\mu \nu \rho} \gamma^{\nu \rho}\right) \epsilon & =0, \\
\left(\frac{i}{2} T_{\mu}^{M} \gamma^{\mu}-\frac{i}{24} H_{\mu \nu \rho}^{M} \gamma^{\mu \nu \rho}\right) \epsilon & =0,
\end{aligned}
$$

where

$$
T_{\mu}^{M} \equiv x_{\alpha}^{M} \partial_{\mu} \hat{\jmath}^{\alpha}
$$

We will consider geometries with $\mathcal{N}=1$ supersymmetry. In 106 the restrictions that the Killing spinor puts on the geometry have been worked out which we will discuss in this section.

On our geometry we introduce a vielbein $\hat{e}^{0}, \ldots, \hat{e}^{5}$, the null-forms 2

$$
e^{-}=\frac{1}{\sqrt{2}}\left(-\hat{e}^{0}+\hat{e}^{5}\right), \quad e^{+}=\frac{1}{\sqrt{2}}\left(\hat{e}^{0}+\hat{e}^{5}\right),
$$

and choose the orientation $\epsilon^{-+1234}=1$. Using the null-vielbein $e^{-}, e^{+}, \hat{e}^{i}$, where $i=1, \ldots, 4$, we can write the metric as

$$
\mathrm{d} \hat{s}_{6}^{2}=2 e^{-} e^{+}+\delta_{i j} \hat{e}^{i} \hat{e}^{j} .
$$

We choose the orientation of the directions perpendicular to the light-cone direction $\epsilon^{i j k l}=\epsilon^{-+i j k l}$ (note that 106 uses the opposite orientation).

The Killing spinor can then be used to construct the bilinears of the geometry, which turn out to be given by $e^{-}$and

$$
e^{-} \wedge I^{1}, \quad e^{-} \wedge I^{2}, \quad e^{-} \wedge I^{3},
$$

where the two-forms $I^{a}$ for $a \in\{1,2,3\}$ take the form

$$
\begin{aligned}
& I^{1}=-\left(\hat{e}^{1} \wedge \hat{e}^{3}+\hat{e}^{2} \wedge \hat{e}^{4}\right), \\
& I^{2}=-\left(\hat{e}^{1} \wedge \hat{e}^{2}-\hat{e}^{3} \wedge \hat{e}^{4}\right), \\
& I^{3}=-\left(\hat{e}^{1} \wedge \hat{e}^{4}-\hat{e}^{2} \wedge \hat{e}^{3}\right) .
\end{aligned}
$$

These two-forms are anti-self-dual on the directions perpendicular to the lightcone direction and satisfy the algebra of the imaginary unit quaternions on a four-manifold with metric $\delta_{i j} \hat{e}^{i} \hat{e}^{j}$ :

$$
\left(I^{a}\right)^{i}{ }_{k}\left(I^{b}\right)^{k}{ }_{j}=-\delta^{a b} \delta_{j}^{i}+\epsilon^{a b}{ }_{c}\left(I^{c}\right)^{i}{ }_{j} .
$$

${ }^{2}$ We relabeled the vielbein of 106 to make notation easier later on. 
Note that the vierbein chosen in 2.14 is special and that the forms $I^{a}$ might look different when another vierbein is used.

The conditions that the gravitino Killing spinor equation imposes on the spacetime geometry can be rewritten as

$$
\begin{aligned}
\nabla_{\mu} e_{\nu}^{-}= & \frac{1}{2} H_{\mu \nu}^{\lambda} e_{\lambda}^{-} \\
\nabla_{\mu}\left(e^{-} \wedge I^{a}\right)_{\nu \lambda \rho}= & \frac{1}{2} H_{\mu \nu}^{\sigma}\left(e^{-} \wedge I^{a}\right)_{\sigma \lambda \rho}+\frac{1}{2} H_{\mu \lambda}^{\sigma}\left(e^{-} \wedge I^{a}\right)_{\nu \sigma \rho} \\
& +\frac{1}{2} H_{\mu \rho}^{\sigma}\left(e^{-} \wedge I^{a}\right)_{\nu \lambda \sigma} .
\end{aligned}
$$

Condition 2.16 implies that the vector $X$ dual to $e^{-}$is a Killing vector. Furthermore, condition 2.16, the $\mu=-$ component of condition 2.17 and the anti-self-duality of $H$ imply that

$$
H=e^{+} \wedge \mathrm{d} e^{-}-\frac{1}{16}\left(I_{k l}^{a} \nabla_{-} I^{b k l}\right) \epsilon_{a b}^{c} I_{c} i j e^{-} \wedge \hat{e}^{i} \wedge \hat{e}^{j}+\frac{1}{6}\left(\mathrm{~d} e^{-}\right)_{-l} \epsilon^{l}{ }_{i j k} \hat{e}^{i} \wedge \hat{e}^{j} \wedge \hat{e}^{k} .
$$

The $\mu=+$ component of condition 2.17 implies that

$$
\nabla_{+} I^{a}=0
$$

and the $\mu=i$ components give further restrictions on the covariant derivatives of $I^{a}$. The tensorini Killing spinor equation implies that the scalars of the tensor multiplets are invariant under the isometry $X$ 106. Combining this equation with the self-duality condition yields that the three-forms of the tensor multiplets take the following form 106 :

$$
H^{M}=\frac{1}{2} H_{-i j}^{M} e^{-} \wedge \hat{e}^{i} \wedge \hat{e}^{j}+T_{i}^{M} e^{-} \wedge e^{+} \wedge \hat{e}^{i}+\frac{1}{6} T_{l}^{M} \epsilon_{i j k}^{l} \hat{e}^{i} \wedge \hat{e}^{j} \wedge \hat{e}^{k},
$$

where $\frac{1}{2} H_{-j k}^{M} e^{-} \wedge \hat{e}^{j} \wedge \hat{e}^{k}$ are self-dual tensors on the directions transverse to the light cone part.

Notice that

$$
\sum_{M} x_{\beta}^{M} T_{i}^{M}=-\Omega_{\alpha \beta} \partial_{i} \hat{\jmath}^{\alpha} .
$$

From 2.3, 2.18 and 2.20 we then find that

$$
\begin{aligned}
\hat{G}^{\alpha}=\hat{\jmath}^{\alpha} e^{+} \wedge \mathrm{d} e^{-}+e^{-} \wedge e^{+} & \wedge \mathrm{d} \hat{\jmath}^{\alpha}+\frac{1}{6}\left[\hat{\jmath}^{\alpha}\left(\mathrm{d} e^{-}\right)_{-l}+\left(\mathrm{d} \hat{\jmath}^{\alpha}\right)_{l}\right] \epsilon_{i j k}^{l} \hat{e}^{i} \wedge \hat{e}^{j} \wedge \hat{e}^{k} \\
& -\left[\frac{1}{8} \hat{\jmath}^{\alpha}\left(I_{k l}^{a} \nabla_{-} I^{b k l}\right) \epsilon_{a b}{ }^{c}\right] e^{-} \wedge I_{c}+e^{-} \wedge H_{\mathrm{SD}}^{\alpha},
\end{aligned}
$$


where

$$
H_{\mathrm{SD}}^{\alpha} \equiv-\frac{1}{2} \Omega^{\alpha \beta} \sum_{M} x_{\beta}^{M} H_{-i j}^{M} \hat{e}^{i} \wedge \hat{e}^{j}
$$

is a self-dual two-form on the directions transverse to the light cone part. Notice that $\hat{\jmath}_{\alpha} H_{\mathrm{SD}}^{\alpha}=0$. Using the Bianchi identity we calculate

$$
\mathcal{L}_{X} \hat{G}^{\alpha}=i_{X} \mathrm{~d} \hat{G}^{\alpha}+\mathrm{d} i_{X} \hat{G}^{\alpha}=\mathrm{d}\left(\hat{\jmath}^{\alpha} \mathrm{d} e^{-}+\mathrm{d} \hat{\jmath}^{\alpha} \wedge e^{-}\right)=0 .
$$

Condition 2.17 combined with the anti-self-duality of $H$ and $I^{a}$ imply that $\mathrm{d}\left(e^{-} \wedge I^{a}\right)=0$ such that also $\mathcal{L}_{X}\left(e^{-} \wedge I^{a}\right)=0$. Hence $X$ generates a symmetry of the full solution.

Summary. Using the vielbein $e^{-}, e^{+}, \hat{e}^{i}$ the field content takes the form 2.12 and 2.22 . Furthermore, we have the two-forms 2.14 that satisfy the condition 2.19 and the $\mu=i$ components of 2.17 . The null-vector $X$ dual to $e^{-}$is a symmetry of the full solution.

\subsubsection{Equations of motion}

From the integrability conditions it follows that if the BPS equations $(2.9)$ and the Bianchi identity are satisfied, the scalar equations of motion and all but the -component of the Einstein equation are obeyed (see appendix 2.A. Hence the set of equations of motion 2.8 reduces to

$$
\begin{aligned}
\hat{R}_{--} & =\frac{1}{4} g_{\alpha \beta} \hat{G}_{-}^{\alpha \rho \lambda} \hat{G}_{-\rho \lambda}^{\beta}-\Omega_{\alpha \beta} \partial_{-} \hat{\jmath}^{\alpha} \partial_{-} \hat{\jmath}^{\beta}, \\
\mathrm{d} \hat{G}^{\alpha} & =0, \\
g_{\alpha \beta} \hat{*} \hat{G}^{\beta} & =-\Omega_{\alpha \beta} \hat{G}^{\beta}, \\
\hat{\jmath}_{\alpha} \hat{\jmath}^{\alpha} & =1 .
\end{aligned}
$$

Note that in the solution of the BPS equations 2.22 , the self-duality condition has already been taken care of.

\subsection{Supersymmetric solutions}

In this section we first use the existence of a null Killing vector to introduce coordinates on the geometry. After this, we rewrite the Bianchi identity and the Einstein equation using these coordinates. 


\subsubsection{Introduction of coordinates}

The existence of a null Killing vector $X$ can be used to introduce coordinates on our spacetime. We implement this here following similar steps as in 77. First a hypersurface $\mathcal{S}$ has to be picked that is nowhere tangent to $X$. One then has to pick a vector $Y$ that satisfies

$$
\hat{g}(Y, X)=1, \quad \hat{g}(Y, Y)=0,
$$

on $\mathcal{S}$. This vector $Y$ needs to be propagated off $\mathcal{S}$ by solving $\mathcal{L}_{X} Y=0$. The properties 2.29 still hold since $X$ is Killing. The vectors $X$ and $Y$ commute, hence they must be tangent to a four-parameter family of two-dimensional surfaces $\Sigma_{2}\left(x^{m}\right)$, where $m=1, \ldots, 4$. The vector $X$ is a null Killing vector, so should be tangent to affinely parametrized null geodesics. Define the coordinate $v$ to be this affine parameter along the geodesics and choose another coordinate $u$ such that $u, v$ are coordinates on the surfaces $\Sigma_{2}$. We can then write

$$
X=\partial_{v}, \quad Y=H\left(\partial_{u}-\frac{1}{2} \mathcal{F} \partial_{v}\right),
$$

for function $\}^{3} H$ and $\mathcal{F}$ independent of $v$. We will assume that (locally) $H>0$ since we can send $u \rightarrow-u$ if necessary. Using the properties of $X$ and $Y$ it can be shown 77 that the metric takes the form

$$
\mathrm{d} \hat{s}_{6}^{2}=2 H^{-1}(\mathrm{~d} u+\beta)\left(\mathrm{d} v+\omega+\frac{1}{2} \mathcal{F}(\mathrm{d} u+\beta)\right)+H \mathrm{~d} s_{4}^{2},
$$

where $\mathrm{d} s_{4}^{2}=h_{m n} \mathrm{~d} x^{m} \mathrm{~d} x^{n}$ is the metric of a base space $\mathcal{B}$ and $\omega, \beta$ are one-forms on $\mathcal{B}\left[77\right.$. The functions $H$ and $\mathcal{F}$, the one-forms $\omega$ and $\beta$ and the metric $h_{m n}$ only depend on $u$ and $x^{m}$ (since $X$ is a Killing vector). The one-forms $e^{-}, e^{+}$in 2.12 take the form

$$
e^{-}=H^{-1}(\mathrm{~d} u+\beta), \quad e^{+}=\mathrm{d} v+\omega+\frac{1}{2} \mathcal{F} H e^{-} .
$$

Notice also that the relation between $\hat{e}^{i}$ and a vierbein $\tilde{e}^{i}$ of $\mathcal{B}$ is given by $\hat{e}^{i}=H^{1 / 2} \tilde{e}^{i}$ which implies $\partial_{\hat{i}}=H^{-1 / 2} \partial_{i}$. From now on, the labels $i, j, k, l$ will refer to the vielbein on the base space.

Let us define anti-self-dual forms on $\mathcal{B}$ by

$$
J^{a}=H^{-1} I^{a} .
$$

\footnotetext{
${ }^{3}$ This notation might be a bit confusing since we already used $H$ for the three-form in the gravity multiplet, but to more easily compare with other literature, we will keep it.
} 
These satisfy the algebra

$$
\left(J^{a}\right)_{p}^{m}\left(J^{b}\right)_{n}^{p}=\epsilon_{c}^{a b}\left(J^{c}\right)^{m}{ }_{n}-\delta^{a b} \delta_{n}^{m},
$$

where the indices $m, n, \ldots$ have been raised with $h^{m n}$. Hence, these two-forms yield an almost hyper-Kähler structure on $\mathcal{B}$.

Following [77] we introduce some more notation. Suppose $\Phi$ is a $p$-form with all its legs on $\mathcal{B}$, but with coefficients depending on $u$ (denote this by $\Phi \in \Lambda^{p}(\mathcal{B})(u)$ ):

$$
\Phi=\frac{1}{p !} \Phi_{m_{1} \ldots m_{p}}(u, x) \mathrm{d} x^{m_{1}} \wedge \ldots \wedge \mathrm{d} x^{m_{p}} .
$$

We then introduce the restricted exterior derivative $\tilde{\mathrm{d}}$ by

$$
\tilde{\mathrm{d}} \Phi \equiv \frac{1}{(p+1) !}(p+1) \frac{\partial}{\partial x^{[n}} \Phi_{\left.m_{1} \ldots m_{p}\right]} \mathrm{d} x^{n} \wedge \mathrm{d} x^{m_{1}} \wedge \ldots \wedge \mathrm{d} x^{m_{p}} .
$$

We also define the operator $\mathcal{D}$ acting on such $p$-forms as

$$
\mathcal{D} \Phi=\tilde{\mathrm{d}} \Phi-\beta \wedge \dot{\Phi},
$$

where $\dot{\Phi}$ is the Lie derivative of $\Phi$ with respect to $\partial_{u}$. Note that

$$
\mathrm{d} \Phi=\mathcal{D} \Phi+H e^{-} \wedge \dot{\Phi} .
$$

Also $\mathcal{D}$ obeys the same product rule on wedge products as the exterior derivative and

$$
\mathcal{D}^{2} \Phi=-\mathcal{D} \beta \wedge \dot{\Phi} .
$$

Using these operators we can derive that

$$
\begin{aligned}
& \mathrm{d} e^{-}=H^{-1} \mathcal{D} \beta+e^{-} \wedge\left(H^{-1} \mathcal{D} H+\dot{\beta}\right), \\
& \mathrm{d} e^{+}=\mathcal{D} \omega+\frac{1}{2} \mathcal{F} \mathcal{D} \beta+H e^{-} \wedge\left(\dot{\omega}+\frac{1}{2} \mathcal{F} \dot{\beta}-\frac{1}{2} \mathcal{D} \mathcal{F}\right) .
\end{aligned}
$$

From these expressions it is straightforward to calculate the spin connection components. They can be found in appendix 2.B

\subsubsection{Supersymmetry and self-duality}

We now derive what the conditions of section 2.2.1 imply in terms of the coordinates we introduced. The three-forms 2.22 become

$$
\begin{aligned}
\hat{G}^{\alpha}=e^{+} & \wedge e^{-} \wedge\left[\hat{\jmath}^{\alpha}\left(H^{-1} \mathcal{D} H+\dot{\beta}\right)-\mathcal{D} \hat{\jmath}^{\alpha}\right]+\hat{\jmath}^{\alpha} H^{-1} e^{+} \wedge \mathcal{D} \beta \\
& +e^{-} \wedge\left[\hat{\jmath}^{\alpha} H \psi-\hat{\jmath}^{\alpha}(\mathcal{D} \omega)^{-}+H_{\mathrm{SD}}^{\alpha}\right]+*_{4} \mathcal{D}\left(\hat{\jmath}^{\alpha} H\right)+H \hat{\jmath}^{\alpha} *_{4} \dot{\beta},
\end{aligned}
$$


where $(\mathcal{D} \omega)^{-} \equiv \frac{1}{2}\left(\mathcal{D} \omega-*_{4} \mathcal{D} \omega\right), *_{4}$ is the Hodge dual on $\mathcal{B}$ and

$$
\psi \equiv-\frac{1}{8} H \epsilon_{a b c} J_{k l}^{a} \partial_{u}\left(J^{b k l}\right) J^{c} .
$$

The self-duality condition 2.27 implies that

$$
\begin{aligned}
\mathcal{D} \beta & =*_{4} \mathcal{D} \beta, \\
H_{\mathrm{SD}}^{\alpha} & =*_{4} H_{\mathrm{SD}}^{\alpha} .
\end{aligned}
$$

Using this one can show that 2.19 is satisfied. The remaining constraints are the $\mu=i$ components of (2.17) and they constrain the covariant derivatives of $J^{a}$ on $\mathcal{B}$. Since $\mathrm{d}\left(e^{-} \wedge I^{a}\right)=0$ we find that

$$
\tilde{\mathrm{d}} J^{a}=\mathcal{L}_{\partial_{u}}\left(\beta \wedge J^{a}\right) .
$$

Together with the fact that the $J^{a}$ satisfy the algebra 2.34 this implies the $\mu=i$ components of 2.17). From 2.45 we conclude that the almost hyper-Kähler structure of $\mathcal{B}$ is not integrable in general.

\subsubsection{Bianchi identity}

We can now substitute expression 2.41 for the three-forms in the Bianchi identity $\mathrm{d} \hat{G}^{\alpha}=0$. Note that this equation is also the equation of motion of the three-forms because of the self-duality condition. Using (2.40), the Bianchi identity reduces to

$$
\tilde{\mathrm{d}}\left(\hat{\jmath}^{\alpha} \psi+\mathcal{G}^{+\alpha}\right)=\mathcal{L}_{\partial_{u}}\left[\beta \wedge\left(\hat{\jmath}^{\alpha} \psi+\mathcal{G}^{+\alpha}\right)+*_{4} \mathcal{D}\left(\hat{\jmath}^{\alpha} H\right)+H \hat{\jmath}^{\alpha} *_{4} \dot{\beta}\right],
$$

and

$$
\mathcal{D} *_{4}\left[\mathcal{D}\left(\hat{\jmath}^{\alpha} H\right)+H \hat{\jmath}^{\alpha} \dot{\beta}\right]+\mathcal{D} \beta \wedge \mathcal{G}^{+\alpha}=0,
$$

where we defined the self-dual two-forms

$$
\mathcal{G}^{+\alpha}=H^{-1}\left[\hat{\jmath}^{\alpha}(\mathcal{D} \omega)^{+}+\frac{1}{2} \hat{\jmath}^{\alpha} \mathcal{F} \mathcal{D} \beta+H_{\mathrm{SD}}^{\alpha}\right],
$$

with $(\mathcal{D} \omega)^{+} \equiv \frac{1}{2}\left(\mathcal{D} \omega+{ }^{*} \mathcal{D} \omega\right)$

\subsubsection{Einstein equation}

We now consider 2.25. In appendix 2.C we use the spin connection components to calculate that

$$
\begin{aligned}
\hat{R}_{--}= & *_{4} \mathcal{D} *_{4}\left(\dot{\omega}+\frac{1}{2} \mathcal{F} \dot{\beta}-\frac{1}{2} \mathcal{D} \mathcal{F}\right)-2\left(\dot{\omega}+\frac{1}{2} \mathcal{F} \dot{\beta}-\frac{1}{2} \mathcal{D} \mathcal{F}\right)^{m} \partial_{u}\left(\beta_{m}\right) \\
& +\frac{1}{2} H^{-2}\left(\mathcal{D} \omega+\frac{1}{2} \mathcal{F} \mathcal{D} \beta\right)^{2}-\frac{1}{2} H h^{m n} \partial_{u}^{2}\left(H h_{m n}\right)-\frac{1}{4} \partial_{u}\left(H h^{m n}\right) \partial_{u}\left(H h_{m n}\right),
\end{aligned}
$$


where for $\Phi_{1}, \Phi_{2} \in \Lambda^{2}(\mathcal{B})(u), \Phi_{1} \cdot \Phi_{2}=\frac{1}{2} \Phi_{1 m n} \Phi_{2}^{m n}$. From 2.41 we find that

$$
\frac{1}{4} g_{\alpha \beta} \hat{G}_{-}^{\alpha \rho \lambda} \hat{G}_{-\rho \lambda}^{\beta}=\frac{1}{2}\left[\psi-H^{-1}(\mathcal{D} \omega)^{-}\right]^{2}+\frac{1}{2} H^{-2} g_{\alpha \beta} H_{\mathrm{SD}}^{\alpha} \cdot H_{\mathrm{SD}}^{\beta} \cdot
$$

The scalars do not depend on $v$ so

$$
\partial_{-} \hat{\jmath}^{\alpha}=H \partial_{u} \hat{\jmath}^{\alpha},
$$

and the Einstein equation becomes

$$
\begin{aligned}
*_{4} \mathcal{D} *_{4}\left(\dot{\omega}+\frac{1}{2} \mathcal{F} \dot{\beta}-\frac{1}{2} \mathcal{D} \mathcal{F}\right)= & 2\left(\dot{\omega}+\frac{1}{2} \mathcal{F} \dot{\beta}-\frac{1}{2} \mathcal{D} \mathcal{F}\right)^{m} \partial_{u}\left(\beta_{m}\right)+\frac{1}{2} H h^{m n} \partial_{u}^{2}\left(H h_{m n}\right) \\
& +\frac{1}{4} \partial_{u}\left(H h^{m n}\right) \partial_{u}\left(H h_{m n}\right)-\frac{1}{2} H^{-2}\left(\mathcal{D} \omega+\frac{1}{2} \mathcal{F} \mathcal{D} \beta\right)^{2} \\
& +\frac{1}{2}\left[\psi-H^{-1}(\mathcal{D} \omega)^{-}\right]^{2}+\frac{1}{2} H^{-2} g_{\alpha \beta} H_{\mathrm{SD}}^{\alpha} \cdot H_{\mathrm{SD}}^{\beta} \\
& -\Omega_{\alpha \beta} H^{2} \partial_{u} \hat{\jmath}^{\alpha} \partial_{u} \hat{\jmath}^{\beta} .
\end{aligned}
$$

\subsubsection{Summary}

We derived the general local form of all supersymmetric solutions of six-dimensional $\mathcal{N}=(1,0)$ supergravity with a gravity multiplet and $n_{T}$ tensor multiplets. The metric is given by 2.31 and the three-forms by 2.41. The equations of motion can be reduced to a set of equations on the base manifold $\mathcal{B}$. The one-form $\beta$ and two-forms $H_{\mathrm{SD}}^{\alpha}$ must obey the self-duality conditions 2.43 and 2.44 . The

Bianchi identity and Einstein equation reduce to 2.46 and 2.47 , and $(2.52)$ respectively. The base manifold must admit an almost hyper-Kähler structure with almost complex structures obeying 2.45.

\subsection{Classes of solutions}

In this section we will, following [77], consider two cases in which the equations derived in the previous section reduce considerably. We first focus on so-called non-twisting solutions, which are solutions in which $\beta=0$. After that we look at $u$-independent solutions and consider their reduction to five dimensions. When the base-space of a $u$-independent solution is chosen to be Gibbons-Hawking and the symmetry of this metric is extended to be a symmetry of the whole solution, we show that it can be expressed in $6+2 n_{T}$ harmonic functions on $\mathbb{R}^{3}$. We then briefly investigate the multi-centered Gibbons-Hawking subclass of these solutions and look at objects as the black string that have a horizon. We finish this section with some examples of pp-wave solutions. 


\subsubsection{Non-twisting solutions}

Non-twisting solutions have $\beta=0$ which highly simplifies the equations. The metric 2.31 and three-forms 2.41 reduce to

$$
\mathrm{d} \hat{s}_{6}^{2}=2 H^{-1} \mathrm{~d} u\left(\mathrm{~d} v+\omega+\frac{1}{2} \mathcal{F} \mathrm{d} u\right)+H \mathrm{~d} s_{4}^{2},
$$

and

$$
\hat{G}^{\alpha}=-e^{+} \wedge \mathrm{d} u \wedge \tilde{\mathrm{d}}\left(H^{-1} \hat{\jmath}^{\alpha}\right)+H^{-1} \mathrm{~d} u \wedge\left[\hat{\jmath}^{\alpha} H \psi-\hat{\jmath}^{\alpha}(\tilde{\mathrm{d}} \omega)^{-}+H_{\mathrm{SD}}^{\alpha}\right]+*_{4} \tilde{\mathrm{d}}\left(\hat{\jmath}^{\alpha} H\right) .
$$

The base space $\mathcal{B}$ with metric $\mathrm{d} s_{4}^{2}$ has to be hyper-Kähler since from 2.45 it follows that

$$
\tilde{\mathrm{d}} J^{a}=0 .
$$

The Bianchi identity, 2.46) and 2.47), reduce to

$$
\tilde{\mathrm{d}}\left(\hat{\jmath}^{\alpha} \psi+\mathcal{G}^{+\alpha}\right)=\mathcal{L}_{\partial_{u}} *_{4} \tilde{\mathrm{d}}\left(\hat{\jmath}^{\alpha} H\right),
$$

and

$$
\tilde{\nabla}^{2}\left(\hat{\jmath}^{\alpha} H\right)=0
$$

where

$$
\mathcal{G}^{+\alpha}=H^{-1}\left[\hat{\jmath}^{\alpha}(\tilde{\mathrm{d}} \omega)^{+}+H_{\mathrm{SD}}^{\alpha}\right] .
$$

Hence the functions $\hat{\jmath}^{\alpha} H$ are harmonic. The Einstein equation 2.52 reduces to

$$
\begin{aligned}
\tilde{\nabla}^{i}(\dot{\omega})_{i}-\frac{1}{2} \tilde{\nabla}^{2} \mathcal{F}= & \frac{1}{2} H h^{m n} \partial_{u}^{2}\left(H h_{m n}\right)+\frac{1}{4} \partial_{u}\left(H h^{m n}\right) \partial_{u}\left(H h_{m n}\right)-\frac{1}{2} H^{-2}(\tilde{\mathrm{d}} \omega)^{2} \\
& +\frac{1}{2}\left[\psi-H^{-1}(\tilde{\mathrm{d}} \omega)^{-}\right]^{2}+\frac{1}{2} H^{-2} g_{\alpha \beta} H_{\mathrm{SD}}^{\alpha} \cdot H_{\mathrm{SD}}^{\beta} \\
& -\Omega_{\alpha \beta} H^{2} \partial_{u} \hat{\jmath}^{\alpha} \partial_{u} \hat{\jmath}^{\beta} .
\end{aligned}
$$

In principle one should be able to solve these equations successively. First pick a hyper-Kähler base space $\mathcal{B}$ and pick harmonic functions on this space for $\hat{\jmath}^{\alpha} H$. The function $H$ then follows by using the identity $\hat{\jmath}_{\alpha} \hat{\jmath}^{\alpha}=1$. The two-form $\mathcal{G}^{+\alpha}$ can be determined by using its self-duality and (2.56). Then $\omega$ can be determined by contracting 2.58 with $\hat{\jmath}_{\alpha}$ which then also gives an expression for $H_{\mathrm{SD}}^{\alpha}$. Lastly, $\mathcal{F}$ can be determined from 2.59).

Solutions that are dependent on $u$ (but not necessarily non-twisting) have been studied in the case of minimal supergravity or in the case with an extra tensor multiplet $81,84,114,116$. To show that one can still do this with an arbitrary number of tensor multiplets, we construct an explicit example of a $u$-dependent solution. 
Flat base space. We will extend the non-twisting solution of [77] with flat base space to a solution with tensor multiplets. As metric on the base space we take

$$
\mathrm{d} s_{4}^{2}=\mathrm{d} r^{2}+\frac{1}{4} r^{2}\left[\left(\sigma^{1}\right)^{2}+\left(\sigma^{2}\right)^{2}+\left(\sigma^{3}\right)^{2}\right],
$$

where $\sigma^{a}, a=1,2,3$, are either the left-invariant $\sigma_{R}^{a}$ or the right-invariant $\sigma_{L}^{a}$ one-forms on the three-sphere: $\mathrm{d} \sigma^{a}=\frac{1}{2} \eta \epsilon_{b c}^{a} \sigma^{b} \wedge \sigma^{c}$ with $\eta=1$ if $\sigma=\sigma_{R}$ and $\eta=-1$ if $\sigma=\sigma_{L}$. We can then take the vierbein

$$
\tilde{e}^{4}=\mathrm{d} r, \quad \tilde{e}^{a}=\frac{r}{2} \sigma^{a} .
$$

If we take the hyper-Kähler structure (in Cartesian coordinates) given by

$$
\begin{aligned}
& J^{1}=-\left(d x^{1} \wedge d x^{3}+d x^{2} \wedge d x^{4}\right), \\
& J^{2}=-\left(d x^{1} \wedge d x^{2}-d x^{3} \wedge d x^{4}\right), \\
& J^{3}=-\left(d x^{1} \wedge d x^{4}-d x^{2} \wedge d x^{3}\right),
\end{aligned}
$$

we have that $\psi=0$. See [102] for the coordinate transformation to express these forms in terms of $\sigma^{a}$. Requiring the two-forms 2.62 to be anti-self-dual imposes the orientation $\tilde{e}^{4} \wedge \tilde{e}^{1} \wedge \tilde{e}^{2} \wedge \tilde{e}^{3}$. For the simple case that $\hat{\jmath}^{\alpha}$ and $H$ only depend on $u$ and $r$, we can write the harmonic functions

$$
\hat{\jmath}^{\alpha} H=P^{\alpha}(u)+\frac{Q^{\alpha}(u)}{r^{2}},
$$

where $P^{\alpha}, Q^{\alpha}$ are arbitrary functions of $u$ that will be fixed by the other equations of motion. From $\hat{\jmath}_{\alpha} \hat{\jmath}^{\alpha}=1$ we find

$$
H=\sqrt{\Omega_{\alpha \beta}\left(P^{\alpha}+\frac{Q^{\alpha}}{r^{2}}\right)\left(P^{\beta}+\frac{Q^{\beta}}{r^{2}}\right)} .
$$

Notice that 2.56 reduces to

$$
\tilde{\mathrm{d}} \mathcal{G}^{+\alpha}=\mathcal{L}_{\partial_{u}} *_{4} \tilde{\mathrm{d}}\left(\hat{\jmath}^{\alpha} H\right) .
$$

Using the self-duality of $\mathcal{G}^{+\alpha}$ we can write

$$
\mathcal{G}^{+\alpha}=C_{b}^{\alpha} \tilde{e}^{4} \wedge \tilde{e}^{b}+\frac{1}{2} C_{b}^{\alpha} \epsilon_{c d}^{b} \tilde{e}^{c} \wedge \tilde{e}^{d},
$$

where we assume that $C_{b}^{\alpha}$ only depend on $u$ and $r$ (to stay in line with 77 ). We can calculate

$$
\tilde{\mathrm{d}} \mathcal{G}^{+\alpha}=\frac{1}{r^{2}}\left[(1-\eta) r C_{b}^{\alpha}+\frac{1}{2} r^{2} \partial_{r}\left(C_{b}^{\alpha}\right)\right] \epsilon_{c d}^{b} \tilde{e}^{4} \wedge \tilde{e}^{c} \wedge \tilde{e}^{d} .
$$


Substitution in 2.65 yields

$$
\begin{aligned}
\frac{1}{r^{2}}\left[(1-\eta) r C_{b}^{\alpha}+\frac{1}{2} r^{2} \partial_{r}\left(C_{b}^{\alpha}\right)\right] \epsilon_{c d}^{b} \tilde{e}^{4} \wedge \tilde{e}^{c} \wedge \tilde{e}^{d} & =\mathcal{L}_{\partial_{u}} *_{4} \tilde{\mathrm{d}}\left(\hat{\jmath}^{\alpha} H\right) \\
& =-2 \frac{\partial_{u} Q^{\alpha}}{r^{3}} \tilde{e}^{1} \wedge \tilde{e}^{2} \wedge \tilde{e}^{3},
\end{aligned}
$$

from which it follows that

$$
\partial_{u} Q^{\alpha}=0, \quad \partial_{r}\left(C_{b}^{\alpha}\right)=2(\eta-1) \frac{1}{r} C_{b}^{\alpha} .
$$

The second equation is solved by

$$
C_{b}^{\alpha}=C_{b}^{\alpha}(u) r^{2(\eta-1)},
$$

for functions $C_{b}^{\alpha}(u)$. Hence

$$
\mathcal{G}^{+\alpha}=C_{b}^{\alpha}(u) r^{2(\eta-1)} \tilde{e}^{4} \wedge \tilde{e}^{b}+\frac{1}{2} C_{b}^{\alpha}(u) r^{2(\eta-1)} \epsilon_{c d}^{b} \tilde{e}^{c} \wedge \tilde{e}^{d} .
$$

Assuming as in 77 that

$$
\omega=W(u, r) \sigma^{3}
$$

we can calculate that

$$
(\tilde{\mathrm{d}} \omega)^{+}=\left(\frac{2}{r^{2}} \eta W+\frac{1}{r} \partial_{r} W\right)\left(\tilde{e}^{1} \wedge \tilde{e}^{2}-\tilde{e}^{3} \wedge \tilde{e}^{4}\right) .
$$

Substitution of 2.63, 2.71 and 2.73 in

$$
(\tilde{\mathrm{d}} \omega)^{+}=H \hat{\jmath}_{\alpha} \mathcal{G}^{+\alpha}
$$

yields

$$
\begin{aligned}
C_{1}^{\alpha}(u) & =C_{2}^{\alpha}(u)=0, \\
W & =W_{1}(u) r^{-2 \eta}+\frac{1}{2} \Omega_{\alpha \beta} C_{3}^{\alpha}(u)\left(\frac{P^{\beta}}{2 \eta} r^{2 \eta}+\frac{Q^{\beta}}{2 \eta-1} r^{2 \eta-2}\right),
\end{aligned}
$$

where $W_{1}$ is yet another arbitrary function of $u$. We then find from 2.63, 2.71 and 2.74) that

$$
\begin{aligned}
H_{\mathrm{SD}}^{\alpha}= & H \mathcal{G}^{+\alpha}-\hat{\jmath}^{\alpha}(\tilde{\mathrm{d}} \omega)^{+} \\
= & {\left[H C_{3}^{\alpha}(u) r^{2(\eta-1)}-\hat{\jmath}^{\alpha} C_{3}^{\beta}(u) r^{2(\eta-1)} \Omega_{\beta \gamma}\left(P^{\gamma}(u)+\frac{Q^{\gamma}(u)}{r^{2}}\right)\right] } \\
& \times\left(\tilde{e}^{1} \wedge \tilde{e}^{2}-\tilde{e}^{3} \wedge \tilde{e}^{4}\right) .
\end{aligned}
$$


Lastly, the Einstein equation 2.59 reduces to

$$
\begin{aligned}
\tilde{\nabla}^{i}(\dot{\omega})_{i}-\frac{1}{2} \tilde{\nabla}^{2} \mathcal{F}=2 H \partial_{u}^{2}(H) & +\partial_{u}(H) \partial_{u}(H)-\frac{1}{2} H^{-2}\left((\tilde{\mathrm{d}} \omega)^{+}\right)^{2} \\
& -\frac{1}{2} H^{-2} \Omega_{\alpha \beta} H_{\mathrm{SD}}^{\alpha} \cdot H_{\mathrm{SD}}^{\beta}-\Omega_{\alpha \beta} H^{2} \partial_{u} \hat{\jmath}^{\alpha} \partial_{u} \hat{\jmath}^{\beta} .
\end{aligned}
$$

Using 2.63, 2.64, 2.72, 2.75 and 2.76), and assuming that $\mathcal{F}=\mathcal{F}(u, r)$ we derive that

$\partial_{r}\left(r^{3} \partial_{r} \mathcal{F}\right)=-2 \Omega_{\alpha \beta} \dot{P}^{\alpha} \dot{P}^{\beta} r^{3}-4 \Omega_{\alpha \beta}\left(P^{\alpha} r^{3}+Q^{\alpha} r\right) \partial_{u}^{2} P^{\beta}+2 \Omega_{\alpha \beta} C_{3}^{\alpha}(u) C_{3}^{\beta}(u) r^{4 \eta-1}$.

Integration of this equation yields

$$
\begin{aligned}
\mathcal{F}=C_{5}(u)- & \frac{1}{2} C_{4}(u) \frac{1}{r^{2}}-\frac{1}{2} \Omega_{\alpha \beta}\left(P^{\alpha} \partial_{u}^{2} P^{\beta}+\frac{1}{2} \dot{P}^{\alpha} \dot{P}^{\beta}\right) r^{2} \\
& +\frac{1}{4 \eta(2 \eta-1)} \Omega_{\alpha \beta} C_{3}^{\alpha}(u) C_{3}^{\beta}(u) r^{4 \eta-2}-2 \Omega_{\alpha \beta} Q^{\alpha} \partial_{u}^{2} P^{\beta} \log (r)
\end{aligned}
$$

for arbitrary functions $C_{4}$ and $C_{5}$. This construction can easily be extended to other hyper-Kähler base spaces.

\subsection{2 u-independent solutions}

A second class of solutions in which the general equations simplify considerably is the class that does not depend on $u$. We can see this as introducing an extra symmetry of the solution. In particular we get an extra Killing vector $\partial_{u}$, which is spacelike when $\mathcal{F}>0$ and timelike when $\mathcal{F}<0$. The three-forms reduce to

$$
\hat{G}^{\alpha}=-H e^{+} \wedge e^{-} \wedge \tilde{\mathrm{d}}\left(H^{-1} \hat{\jmath}^{\alpha}\right)+\hat{\jmath}^{\alpha} H^{-1} e^{+} \wedge \tilde{\mathrm{d}} \beta+e^{-} \wedge\left[-\hat{\jmath}^{\alpha}(\tilde{\mathrm{d}} \omega)^{-}+H_{\mathrm{SD}}^{\alpha}\right]+*_{4} \tilde{\mathrm{d}}\left(\hat{\jmath}^{\alpha} H\right) \text {. }
$$

The base space has to be hyper-Kähler since from 2.45

$$
\tilde{\mathrm{d}} J^{a}=0
$$

and $\beta$ has self-dual curvature:

$$
\tilde{\mathrm{d}} \beta=*_{4} \tilde{\mathrm{d}} \beta .
$$

The Bianchi identity, 2.46 and 2.47), and Einstein equation 2.52 reduce to respectively

$$
\begin{aligned}
\tilde{\mathrm{d}} \mathcal{G}^{+\alpha} & =0, \\
\tilde{\mathrm{d}} *_{4} \tilde{\mathrm{d}}\left(\hat{\jmath}^{\alpha} H\right) & =-\tilde{\mathrm{d}} \beta \wedge \mathcal{G}^{+\alpha}, \\
\tilde{\nabla}^{2} \mathcal{F} & =\Omega_{\alpha \beta} \mathcal{G}^{+\alpha} \cdot \mathcal{G}^{+\beta},
\end{aligned}
$$


where

$$
\mathcal{G}^{+\alpha}=H^{-1}\left[\hat{\jmath}^{\alpha}(\tilde{\mathrm{d}} \omega)^{+}+\frac{1}{2} \hat{\jmath}^{\alpha} \mathcal{F} \tilde{\mathrm{d}} \beta+H_{\mathrm{SD}}^{\alpha}\right],
$$

and we have used that

$$
\Omega_{\alpha \beta} \mathcal{G}^{+\alpha} \cdot \mathcal{G}^{+\beta}=H^{-2}\left[(\tilde{\mathrm{d}} \omega)^{+}+\frac{1}{2} \mathcal{F} \tilde{\mathrm{d}} \beta\right] \cdot\left[(\tilde{\mathrm{d}} \omega)^{+}+\frac{1}{2} \mathcal{F} \tilde{\mathrm{d}} \beta\right]-H^{-2} g_{\alpha \beta} H_{\mathrm{SD}}^{\alpha} \cdot H_{\mathrm{SD}}^{\beta} .
$$

When the Killing vector $\partial_{u}$ is spacelike, the $u$-direction can be compactified on a circle and we can reduce the solution to five dimensions. This will be done in the subsequent section. In section 2.4.4 we then take the base space $\mathcal{B}$ to be GibbonsHawking [107] which has yet another Killing vector. Assuming that this symmetry extends to the whole solution, we solve the equations of motion completely.

\subsubsection{Reduction to five dimensions}

When one considers a $u$-independent solution with $\mathcal{F}$ positive such that $\partial_{u}$ is a spacelike Killing vector, one can compactify this direction on a circle and do a Kaluza-Klein reduction, as introduced in section 1.2.3.1 to obtain a five-dimensional solution. The six-dimensional metric reduces to the five-dimensional metric $\mathrm{d} s_{5}^{2}$, a Kaluza-Klein vector $A^{0}$ and a scalar $X^{0}$. The three-forms $\hat{G}^{\alpha}$ reduce to three-forms $G^{\alpha}$ and two-forms $F^{\alpha}$ that are related to each other since the $\hat{G}^{\alpha}$ obey a self-duality condition. The scalars $\hat{\jmath}^{\alpha}$ reduce to scalars $X^{\alpha}$. Reducing the six-dimensional theory to five dimensions thus results in five-dimensional supergravity coupled to $n_{T}+1$ vector multiplets. We can express the six-dimensional data in terms of five-dimensional data by $37 \mathbf{4}^{4}$

$$
\begin{aligned}
\mathrm{d} \hat{s}_{6}^{2} & =r^{2}\left(\mathrm{~d} u+A^{0}\right)^{2}+r^{-2 / 3} \mathrm{~d} s_{5}^{2}, \\
\hat{G}^{\alpha} & =G^{\alpha}-F^{\alpha} \wedge\left(\mathrm{d} u+A^{0}\right), \\
\hat{\jmath}^{\alpha} & =r^{-2 / 3} X^{\alpha}, \\
r^{-4 / 3} & =X^{0},
\end{aligned}
$$

where

$$
\mathrm{d} s_{5}^{2}=-f^{2}(\mathrm{~d} v+\omega)^{2}+f^{-1} \mathrm{~d} s_{4}^{2}, \quad G^{\alpha}=\mathrm{d} B^{\alpha}+A^{\alpha} \wedge F^{0},
$$

for a function $f$ and two-forms $B^{\alpha}$. The scalars $X^{I}, I \in\left\{0,1, \ldots, n_{T}+1\right\}$, are the so-called very special coordinates. These are are $n_{T}+2$ real coordinates that

${ }^{4}$ With this ansatz the five-dimensional field content is expressed in Einstein frame. 
describe an $\left(n_{T}+2\right)$-dimensional manifold in which the scalar manifold is given by the hypersurface 117

$$
\mathcal{N} \equiv \frac{1}{3 !} C_{I J K} X^{I} X^{J} X^{K}=1 .
$$

Here $C_{I J K}$ is a constant symmetric tensor and $\mathcal{N}$ is the so-called cubic potential. The potential $\mathcal{N}$ in terms of six-dimensional data is given by 37

$$
\mathcal{N}=\Omega_{\alpha \beta} X^{0} X^{\alpha} X^{\beta}
$$

It is straightforward to derive that

$$
\begin{aligned}
\mathrm{d} s_{5}^{2} & =-\left(\mathcal{F} H^{2}\right)^{-2 / 3}(\mathrm{~d} v+\omega)^{2}+\left(\mathcal{F} H^{2}\right)^{1 / 3} \mathrm{~d} s_{4}^{2}, \\
X^{0} & =\left(\mathcal{F} H^{-1}\right)^{-2 / 3}, \\
X^{\alpha} & =\left(\mathcal{F} H^{-1}\right)^{1 / 3} \hat{\jmath}^{\alpha}, \\
F^{0} & =\tilde{\mathrm{d}}\left[\beta+\mathcal{F}^{-1}(\mathrm{~d} v+\omega)\right], \\
F^{\alpha} & =\tilde{\mathrm{d}}\left[H^{-1} \hat{\jmath}^{\alpha}(\mathrm{d} v+\omega)\right]-H^{-1}\left[\hat{\jmath}^{\alpha}(\tilde{\mathrm{d}} \omega)^{+}+\frac{1}{2} \hat{\jmath}^{\alpha} \mathcal{F} \tilde{\mathrm{d}} \beta+H_{\mathrm{SD}}^{\alpha}\right] .
\end{aligned}
$$

Note that the field strengths can be written as

$$
F^{I}=\tilde{\mathrm{d}}\left[X^{I} f(\mathrm{~d} v+\omega)\right]+\Theta^{I}
$$

where

$$
\Theta^{0}=\tilde{\mathrm{d}} \beta, \quad \Theta^{\alpha}=-\mathcal{G}^{\alpha},
$$

are self-dual tensors. Also 2.83 implies that the two-forms $\Theta^{I}$ are closed. Using

$$
X_{I} \equiv \frac{1}{6} C_{I J K} X^{J} X^{K}
$$

we find that

$$
X_{I} \Theta^{I}=-\frac{2}{3} f(\tilde{\mathrm{d}} \omega)^{+} .
$$

Furthermore, we find that 2.84 reduces to

$$
\tilde{\nabla}^{2}\left(f^{-1} X_{\alpha}\right)=\frac{1}{6} C_{\alpha J K} \Theta^{J} \cdot \Theta^{K},
$$

and that the Einstein equation 2.85 reduces to

$$
\tilde{\nabla}^{2}\left(f^{-1} X_{0}\right)=\frac{1}{6} C_{0 J K} \Theta^{J} \cdot \Theta^{K} .
$$

This implies that we find ourselves exactly in the timelike class of five-dimensional solutions of 104,118. Thus every solution of five-dimensional supergravity coupled 
to an arbitrary number of vector multiplets in the timelike class that has a cubic potential of the form

$$
\mathcal{N}=\frac{1}{3 !} C_{I J K} X^{I} X^{J} X^{K}=\Omega_{\alpha \beta} X^{0} X^{\alpha} X^{\beta},
$$

can be uplifted to six dimensions. An interesting remark is that classical M-theory solutions do not have cubic potentials of this form. To lift them up, one also has to take into account one-loop corrections with massive Kaluza-Klein modes running in the loop 37,119 .

Minimal five-dimensional supergravity. After the reduction we always end up with at least one vector multiplet in five dimensions. However, one can truncate the reduction of minimal supergravity in six dimensions to minimal five-dimensional supergravity 77. We get minimal supergravity when we set the three-forms of the tensor multiplets $H^{M}=0$ for $M=1, \ldots, n_{T}$ and furthermore set $\hat{\jmath}^{\alpha}=0$ for $\alpha=2, \ldots, n_{T}+1$ and $\hat{\jmath}^{1}=1$. The only three-form that is non-zero is $\hat{G}^{1}$. We can truncate the reduced theory to minimal supergravity by getting rid of the scalars, which can be done by setting $\mathcal{F}=H$ such that $X^{0}=X^{1}=1$. Consistency of 2.84 and 2.85 then enforces $\tilde{\mathrm{d}} \beta=-\mathcal{G}^{+1}$ or

$$
\tilde{\mathrm{d}} \beta=-\frac{2}{3} H^{-1}(\tilde{\mathrm{d}} \omega)^{+} .
$$

This implies that

$$
F \equiv F^{0}=F^{1}=\tilde{\mathrm{d}}\left[\beta+\mathcal{F}^{-1}(\mathrm{~d} v+\omega)\right] .
$$

Introducing $G^{+} \equiv f(\tilde{\mathrm{d}} \omega)^{+}$, we find that

$$
\tilde{\mathrm{d}} G^{+}=0
$$

and that 2.99 reduces to

$$
\tilde{\nabla}^{2}\left(f^{-1}\right)=\frac{4}{9} G^{+} \cdot G^{+}
$$

This means that we find ourselves in the timelike class of minimal five-dimensional supergravity $1025^{5}$

The null class of minimal five-dimensional supergravity arises from reducing non-twisting solutions of minimal six-dimensional supergravity that have a GibbonsHawking base space 77 .

\footnotetext{
${ }^{5}$ They use a different normalization of the field strength: $F_{\text {here }}=\frac{2}{\sqrt{3}} F_{\text {there }}$.
} 


\subsubsection{Gibbons-Hawking base space}

We now consider $u$-independent solutions with a Gibbons-Hawking base space [107. This is the most general hyper-Kähler four-manifold admitting a Killing vector field $\partial_{\psi}{ }^{6}$ preserving the three complex structures 120.7 It has the metric

$$
\mathrm{d} s_{4}^{2}=H_{2}^{-1}\left(\mathrm{~d} \psi+\chi_{a} \mathrm{~d} x^{a}\right)^{2}+H_{2} \delta_{a b} \mathrm{~d} x^{a} \mathrm{~d} x^{b},
$$

where $a=1,2,3, \chi_{a}$ and $H_{2}$ are independent of $\psi$ and

$$
\nabla^{2} H_{2}=0, \quad \vec{\nabla} \times \vec{\chi}=\vec{\nabla} H_{2} .
$$

We take $\nabla$ with respect to three-dimensional flat space.

We now obtain all solutions in the case the symmetry $\partial_{\psi}$ of the base space is extended to a symmetry of the full solution. This was done in 102 for minimal five-dimensional supergravity, in [77] for minimal six-dimensional supergravity and in 118 for five-dimensional supergravity coupled to an arbitrary number of vector multiplets, so we will be quite brief here.

We can choose the vierbein

$$
\tilde{e}^{4}=H_{2}^{-1 / 2}\left(\mathrm{~d} \psi+\chi_{a} \mathrm{~d} x^{a}\right), \quad \tilde{e}^{a}=H_{2}^{1 / 2} \mathrm{~d} x^{a} .
$$

Anti-self-duality of the complex structure forms implies that the volume form is given by $\tilde{e}^{4} \wedge \tilde{e}^{1} \wedge \tilde{e}^{2} \wedge \tilde{e}^{3}$. We can decompose the one-forms

$$
\beta=\beta_{0}\left(\mathrm{~d} \psi+\chi_{a} \mathrm{~d} x^{a}\right)+\beta_{a} \mathrm{~d} x^{a}, \quad \omega=\omega_{0}\left(\mathrm{~d} \psi+\chi_{a} \mathrm{~d} x^{a}\right)+\omega_{a} \mathrm{~d} x^{a},
$$

where $\beta_{0}, \beta_{a}, \omega_{0}$ and $\omega_{a}$ are functions on $\mathbb{R}^{3}$. Solving 2.82 results in

$$
\beta_{0}=H_{2}^{-1} H_{3}, \quad \vec{\nabla} \times \vec{\beta}=-\vec{\nabla} H_{3},
$$

with $H_{3}$ an arbitrary harmonic function on $\mathbb{R}^{3}$. The two-form $\mathcal{G}^{+\alpha}$ is self-dual so it has to be of the form

$$
\mathcal{G}^{+\alpha}=-\frac{1}{2} C_{b}^{\alpha} \tilde{e}^{4} \wedge \tilde{e}^{b}-\frac{1}{4} C_{b}^{\alpha} \epsilon_{c d}^{b} \tilde{e}^{c} \wedge \tilde{e}^{d} .
$$

Solving 2.83 results in

$$
\vec{C}^{\alpha}=2 \vec{\nabla}\left(H_{2}^{-1} H_{4}^{\alpha}\right)
$$

\footnotetext{
${ }^{6}$ Notice that this $\psi$ is not related to the $\psi$ in terms of the complex structures. We can safely do this since from 2.42 we see that $\psi=0$ for this class of solutions.

${ }^{7}$ The solution in 107 is actually a subclass of these manifolds, but we refer here to its generalization as Gibbons-Hawking.
} 
with $H_{4}^{\alpha}$ arbitrary harmonic functions on $\mathbb{R}^{3}$. Using this result one can solve 2.84, which results in

$$
\hat{\jmath}^{\alpha} H=H_{1}^{\alpha}-H_{2}^{-1} H_{3} H_{4}^{\alpha},
$$

where $H_{1}^{\alpha}$ are arbitrary harmonic functions on $\mathbb{R}^{3}$. Using $\hat{\jmath}_{\alpha} \hat{\jmath}^{\alpha}=1$ we find

$$
H=\sqrt{\Omega_{\alpha \beta}\left(H_{1}^{\alpha}-H_{2}^{-1} H_{3} H_{4}^{\alpha}\right)\left(H_{1}^{\beta}-H_{2}^{-1} H_{3} H_{4}^{\beta}\right)} .
$$

With the solution of $\mathcal{G}^{+\alpha}, 2.85$ can be solved and yields

$$
\mathcal{F}=-H_{5}+H_{2}^{-1} \Omega_{\alpha \beta} H_{4}^{\alpha} H_{4}^{\beta},
$$

with $H_{5}$ an arbitrary harmonic function on $\mathbb{R}^{3}$. Now, using that

$$
\begin{aligned}
\hat{\jmath}_{\alpha} \mathcal{G}^{+\alpha} & =-\hat{\jmath}_{\alpha} \nabla_{b}\left(H_{2}^{-1} H_{4}^{\alpha}\right) \tilde{e}^{4} \wedge \tilde{e}^{b}-\frac{1}{2} \hat{\jmath}_{\alpha} \nabla_{b}\left(H_{2}^{-1} H_{4}^{\alpha}\right) \epsilon_{c d}^{b} \tilde{e}^{c} \wedge \tilde{e}^{d} \\
& =H^{-1}(\tilde{\mathrm{d}} \omega)^{+}+\frac{1}{2} \mathcal{F} H^{-1} \tilde{\mathrm{d}} \beta
\end{aligned}
$$

we get an equation for $\omega$ :

$$
\begin{aligned}
H_{2} \vec{\nabla} \omega_{0}-\omega_{0} \vec{\nabla} H_{2}-\vec{\nabla} \times \vec{\omega}=2 \Omega_{\alpha \beta}\left(H_{1}^{\alpha} H_{2}-H_{3} H_{4}^{\alpha}\right) \vec{\nabla}\left(H_{2}^{-1} H_{4}^{\beta}\right) \\
+\left(H_{5} H_{2}-\Omega_{\alpha \beta} H_{4}^{\alpha} H_{4}^{\beta}\right) \vec{\nabla}\left(H_{2}^{-1} H_{3}\right) .
\end{aligned}
$$

Taking the divergence of this equation yields an integrability equation that can be solved for $\omega_{0}$ :

$$
\omega_{0}=H_{6}-H_{2}^{-2} H_{3} \Omega_{\alpha \beta} H_{4}^{\alpha} H_{4}^{\beta}+H_{2}^{-1} \Omega_{\alpha \beta} H_{1}^{\alpha} H_{4}^{\beta}+\frac{1}{2} H_{2}^{-1} H_{3} H_{5},
$$

with $H_{6}$ an arbitrary harmonic function on $\mathbb{R}^{3}$. Substitution of this in 2.116 gives an equation that determines $\vec{\omega}$ up to a gradient (and this can be eliminated by shifting $v)$ :

$$
\vec{\nabla} \times \vec{\omega}=\Omega_{\alpha \beta}\left(H_{4}^{\alpha} \vec{\nabla} H_{1}^{\beta}-H_{1}^{\alpha} \vec{\nabla} H_{4}^{\beta}\right)+H_{2} \vec{\nabla} H_{6}-H_{6} \vec{\nabla} H_{2}+\frac{1}{2} H_{3} \vec{\nabla} H_{5}-\frac{1}{2} H_{5} \vec{\nabla} H_{3} .
$$

From the definition of $\mathcal{G}^{+\alpha}$ we then find that

$$
H_{\mathrm{SD}}^{\alpha}=-D_{b}^{\alpha} \tilde{e}^{4} \wedge \tilde{e}^{b}-\frac{1}{2} D_{b}^{\alpha} \epsilon_{c d}^{b} \tilde{e}^{c} \wedge \tilde{e}^{d}
$$

where

$$
\vec{D}^{\alpha} \equiv H \vec{\nabla}\left(H_{2}^{-1} H_{4}^{\alpha}\right)-\hat{\jmath}^{\alpha} H \hat{\jmath}_{\beta} \vec{\nabla}\left(H_{2}^{-1} H_{4}^{\beta}\right)
$$


We now consider the so-called multi-centered Gibbons-Hawking subclass of these solutions. We introduce the notation

$$
\begin{gathered}
\mathbb{H} \equiv\left(H_{1}^{\alpha}, H_{2}, H_{3}, H_{4}^{\alpha}, H_{5}, H_{6}\right), \quad \Gamma_{A} \equiv\left(\mu_{A}^{\alpha}, m_{A}, q_{A}, p_{A}^{\alpha}, n_{A}, j_{A}\right), \\
\Gamma_{\infty} \equiv\left(\mu_{\infty}^{\alpha}, m_{\infty}, q_{\infty}, p_{\infty}^{\alpha}, n_{\infty}, j_{\infty}\right),
\end{gathered}
$$

where $A=1, \ldots, N$ and all the components of the vectors $\Gamma_{A}$ and $\Gamma_{\infty}$ are constants. We then take the harmonic functions of the form

$$
\mathbb{H}=\Gamma_{\infty}+\sum_{A} \frac{\Gamma_{A}}{\left|\vec{x}-\vec{x}_{A}\right|} .
$$

Although every set of centers $\vec{x}_{A}$ describes a solution, there will typically be Dirac string-like singularities. Imposing the absence of these singularities gives a constraint on the relative positions, see $99,121,122]$. This arises from requiring $\vec{\omega}$ to be globally defined, which implies that $\mathrm{d}^{2} \vec{\omega}=0$. If we define the symplectic product \langle\rangle working on vectors of the form $v=\left(v_{1}^{\alpha}, v_{2}, v_{3}, v_{4}^{\alpha}, v_{5}, v_{6}\right)$ via

$$
\langle v, w\rangle=\Omega_{\alpha \beta} v_{4}^{\alpha} w_{1}^{\beta}-\Omega_{\alpha \beta} v_{1}^{\alpha} w_{4}^{\beta}+v_{2} w_{6}-v_{6} w_{2}+\frac{1}{2}\left(v_{3} w_{5}-v_{5} w_{3}\right),
$$

we can rewrite 2.118 as

$$
*_{3} \mathrm{~d} \vec{\omega}=\langle\mathbb{H}, \mathrm{d} \mathbb{H}\rangle \text {. }
$$

Taking $\mathrm{d} *_{3}$ on both sides leads to

$$
\sum_{B \neq A} \frac{\left\langle\Gamma_{A}, \Gamma_{B}\right\rangle}{\left|\vec{x}_{A}-\vec{x}_{B}\right|}=\left\langle\Gamma_{\infty}, \Gamma_{A}\right\rangle, \quad A=1, \ldots, N .
$$

These are usually referred to as Bubble equations since they control the size of the two-cycles or bubbles in the Gibbons-Hawking base space [123.

Let $S p\left(6+2 n_{T}, \mathbb{R}\right)$ denote the symplectic group that preserves the symplectic product \langle\rangle . A linear combination of harmonic functions is still harmonic, hence sending $\mathbb{H} \rightarrow g \mathbb{H}$ with $g \in S p\left(6+2 n_{T}, \mathbb{R}\right)$ sends a solution to a solution, preserving regularity. This symplectic group was earlier noticed for minimal supergravity in 108 .

Summary. The most general $u$-independent solution with a Gibbons-Hawking base space whose Killing vector field extends to a symmetry of the full solution is determined by $6+2 n_{T}$ harmonic functions $H_{1}^{\alpha}, H_{2}, H_{3}, H_{4}^{\alpha}, H_{5}$ and $H_{6}$ on $\mathbb{R}^{3}$. Its metric is given by

$$
\begin{aligned}
\mathrm{d} \hat{s}_{6}^{2} & =2 H^{-1}(\mathrm{~d} u+\beta)\left(\mathrm{d} v+\omega+\frac{1}{2} \mathcal{F}(\mathrm{d} u+\beta)\right)+H \mathrm{~d} s_{4}^{2}, \\
\mathrm{~d} s_{4}^{2} & =H_{2}^{-1}\left(\mathrm{~d} \psi+\chi_{a} \mathrm{~d} x^{a}\right)^{2}+H_{2} \delta_{a b} \mathrm{~d} x^{a} \mathrm{~d} x^{b},
\end{aligned}
$$


where

$$
\begin{aligned}
\vec{\nabla} \times \vec{\chi} & =\vec{\nabla} H_{2}, \\
H & =\sqrt{\Omega_{\alpha \beta}\left(H_{1}^{\alpha}-H_{2}^{-1} H_{3} H_{4}^{\alpha}\right)\left(H_{1}^{\beta}-H_{2}^{-1} H_{3} H_{4}^{\beta}\right)}, \\
\mathcal{F} & =-H_{5}+H_{2}^{-1} \Omega_{\alpha \beta} H_{4}^{\alpha} H_{4}^{\beta} .
\end{aligned}
$$

The one-forms are decomposed as

$$
\beta=\beta_{0}\left(\mathrm{~d} \psi+\chi_{a} \mathrm{~d} x^{a}\right)+\beta_{a} \mathrm{~d} x^{a}, \quad \omega=\omega_{0}\left(\mathrm{~d} \psi+\chi_{a} \mathrm{~d} x^{a}\right)+\omega_{a} \mathrm{~d} x^{a},
$$

with the coefficients $\beta_{0}, \beta_{a}, \omega_{0}$ and $\omega_{a}$ given by

$$
\begin{aligned}
\beta_{0}= & H_{2}^{-1} H_{3}, \\
\vec{\nabla} \times \vec{\beta}= & -\vec{\nabla} H_{3}, \\
\omega_{0}= & H_{6}-H_{2}^{-2} H_{3} \Omega_{\alpha \beta} H_{4}^{\alpha} H_{4}^{\beta}+H_{2}^{-1} \Omega_{\alpha \beta} H_{1}^{\alpha} H_{4}^{\beta}+\frac{1}{2} H_{2}^{-1} H_{3} H_{5}, \\
\vec{\nabla} \times \vec{\omega}= & \Omega_{\alpha \beta}\left(H_{4}^{\alpha} \vec{\nabla} H_{1}^{\beta}-H_{1}^{\alpha} \vec{\nabla} H_{4}^{\beta}\right)+H_{2} \vec{\nabla} H_{6}-H_{6} \vec{\nabla} H_{2} \\
& +\frac{1}{2} H_{3} \vec{\nabla} H_{5}-\frac{1}{2} H_{5} \vec{\nabla} H_{3} .
\end{aligned}
$$

The three-forms are equal to

$\hat{G}^{\alpha}=-H e^{+} \wedge e^{-} \wedge \tilde{\mathrm{d}}\left(H^{-1} \hat{\jmath}^{\alpha}\right)+\hat{\jmath}^{\alpha} H^{-1} e^{+} \wedge \tilde{\mathrm{d}} \beta+e^{-} \wedge\left[-\hat{\jmath}^{\alpha}(\tilde{\mathrm{d}} \omega)^{-}+H_{\mathrm{SD}}^{\alpha}\right]+*_{4} \tilde{\mathrm{d}}\left(\hat{\jmath}^{\alpha} H\right)$

where

$$
\begin{aligned}
e^{-} & =H^{-1}(\mathrm{~d} u+\beta), \\
e^{+} & =\mathrm{d} v+\omega+\frac{1}{2} \mathcal{F} H e^{-}, \\
H_{\mathrm{SD}}^{\alpha} & =-D_{b}^{\alpha}\left(\mathrm{d} \psi+\chi_{a} \mathrm{~d} x^{a}\right) \wedge \mathrm{d} x^{b}-\frac{1}{2} H_{2} D_{b}^{\alpha} \epsilon_{c d}^{b} \mathrm{~d} x^{c} \wedge \mathrm{d} x^{d}, \\
\vec{D}^{\alpha} & =H \vec{\nabla}\left(H_{2}^{-1} H_{4}^{\alpha}\right)-\hat{\jmath}^{\alpha} H \hat{\jmath}_{\beta} \vec{\nabla}\left(H_{2}^{-1} H_{4}^{\beta}\right) .
\end{aligned}
$$

Lastly, the scalars are given by

$$
\hat{\jmath}^{\alpha}=\frac{H_{1}^{\alpha}-H_{2}^{-1} H_{3} H_{4}^{\alpha}}{H} .
$$

When the harmonic functions are taken of the form 2.122 , one has to impose the bubble equations 2.125 in order to avoid Dirac string-like singularities. 


\subsubsection{Black strings and other objects with a horizon}

In 95 it is shown that in supergravity coupled to tensor multiplets, near-horizon geometries of black objects are locally either $\mathbb{R}^{1,1} \times \mathrm{T}^{4}, \mathbb{R}^{1,1} \times \mathrm{K} 3$ or $\mathrm{AdS}_{3} \times \mathrm{S}^{3}$. In this section we will consider some examples of black objects with near-horizon geometry $\mathrm{AdS}_{3} \times \mathrm{S}^{3}$. These can correspond to a black string or the uplift of a black ring or black lens.

Black string. When taking a solution of section 2.4.4, compactifying the $u$ direction on a circle, taking the harmonic functions of the form 2.122 with only one center $\vec{x}_{1}=0$ and requiring the metric to asymptote to $\mathbb{R} \times \mathrm{S}^{1} \times \mathbb{R}^{4} / \mathbb{Z}_{m}$, we find a generalization of the single black string solution in [108]. Perhaps the most interesting case is $m=1$, which at infinity corresponds to a black string wrapped around a circle times a flat 5 d Minkowski spacetime. For $m \neq 1$ one gets ALE spaces.

The string is wound around the $u$-direction and becomes a black hole after reduction on the $u$-circle. In appendix 2.D we present the expressions for the one-forms $\vec{\chi}, \vec{\beta}$ and $\vec{\omega}$. In addition, we derive that to get the right asymptotics for the metric, we need

$$
\Gamma_{\infty}=\left(\mu_{\infty}^{\alpha}, 0,0,0,-1, \frac{1}{2} \frac{q}{m}-\frac{1}{m} \Omega_{\alpha \beta} \mu_{\infty}^{\alpha} p^{\beta}\right)
$$

with

$$
\Omega_{\alpha \beta} \mu_{\infty}^{\alpha} \mu_{\infty}^{\beta}=1
$$

Using spherical coordinates $r, \theta, \phi$ for the $\mathbb{R}^{3}$ part, the metric of the solution is then given by

$$
\begin{aligned}
\mathrm{d} \hat{s}_{6}^{2}=2\left(1+2 \frac{\Omega_{\alpha \beta} \mu_{\infty}^{\alpha} \tilde{Q}^{\beta}}{4 \sqrt{2} r}+\frac{\Omega_{\alpha \beta} \tilde{Q}^{\alpha} \tilde{Q}^{\beta}}{32 r^{2}}\right)^{-1 / 2} \mathrm{~d} u^{\prime} \\
\times\left[\mathrm{d} v+\frac{J_{\psi}}{8 r}(\mathrm{~d} \psi+m \cos (\theta) \mathrm{d} \phi)+\frac{1}{2}\left(1+\frac{Q}{4 r}\right) \mathrm{d} u^{\prime}\right] \\
+\left(1+2 \frac{\Omega_{\alpha \beta} \mu_{\infty}^{\alpha} \tilde{Q}^{\beta}}{4 \sqrt{2} r}+\frac{\Omega_{\alpha \beta} \tilde{Q}^{\alpha} \tilde{Q}^{\beta}}{32 r^{2}}\right)^{1 / 2} \\
\times\left[\frac{r}{m}(\mathrm{~d} \psi+m \cos (\theta) \mathrm{d} \phi)^{2}+\frac{m}{r} \mathrm{~d} r^{2}+m r \mathrm{~d} \Omega_{2}^{2}\right],
\end{aligned}
$$

where we defined $u^{\prime}=u+\frac{q}{m} \psi$. To make this transformation well-defined, we have to impose

$$
\frac{4 \pi q}{l m} \in \mathbb{Z}
$$


where $l$ is the length of the $u$-circle. We also defined

$$
\begin{aligned}
\tilde{Q}^{\alpha} & \equiv 4 \sqrt{2}\left(\mu^{\alpha}-\frac{1}{m} q p^{\alpha}\right), \\
Q & \equiv 4\left(-n+\frac{1}{m} \Omega_{\alpha \beta} p^{\alpha} p^{\beta}\right), \\
J_{\psi} & \equiv 8\left(j-\frac{1}{m^{2}} q \Omega_{\alpha \beta} p^{\alpha} p^{\beta}+\frac{1}{m}\left(\Omega_{\alpha \beta} \mu^{\alpha} p^{\beta}+\frac{1}{2} q n\right)\right) .
\end{aligned}
$$

The near-horizon geometry of this solution is a direct product of an extremal BTZ black hole and a round $S^{3} / \mathbb{Z}_{m}$. The entropy of the black string is given by

$$
S=\frac{A}{4 G^{(6)}}=2 \pi \sqrt{\frac{1}{2} m Q \Omega_{\alpha \beta} \tilde{Q}^{\alpha} \tilde{Q}^{\beta}-J_{\psi}^{2}},
$$

where we used conventions in which $G^{(6)}=\frac{l \pi}{4}$.

These black strings can be embedded in F-theory and we consider the case $m=1$ for simplicity. We take an F-theory background $\mathbb{R} \times \mathrm{S}^{1} \times \mathbb{R}^{4} \times \mathrm{CY}_{3}$, where $\mathrm{CY}_{3}$ is a smooth elliptically fibered Calabi-Yau three-fold $\pi: \mathrm{CY}_{3} \rightarrow B$. The solution corresponds to a D3-brane wrapped on $\mathrm{S}^{1} \times C$, where $C \subset B$ is a curve. We have the set of vertical divisors $D_{\alpha} \equiv \pi^{-1}\left(D_{\alpha}^{b}\right)$, where $D_{\alpha}^{b}$ are divisors of $B$ that are chosen such that

$$
\Omega_{\alpha \beta}=\int_{B} \omega_{\alpha} \wedge \omega_{\beta}
$$

for $\omega_{\alpha}$ the two-form classes Poincaré dual to $D_{\alpha}$. We can then write $C=q^{\alpha} \omega_{\alpha}$ for the form Poincaré dual to the curve $C$. The strings that one gets after compactification on $\mathrm{CY}_{3}$ carry $n$ units of momentum along the circle. There is also an $S O(4) \equiv$ $S U(2)_{L} \times S U(2)_{R}$ symmetry from rotations in the $\mathbb{R}^{4}$ plane transverse to the D3-brane. The entropy corresponding to a single string to leading order in the large charge limit is given by 76

$$
S=2 \pi \sqrt{\frac{1}{2} n \Omega_{\alpha \beta} q^{\alpha} q^{\beta}-J^{2}},
$$

where $J$ is the eigenvalue corresponding to the $U(1)_{L} \subset S U(2)_{L}$ symmetry in the convention that it is half-integer valued. The microscopic formula 2.140 is only valid in the Cardy limit where $n$ is much larger than the other charges. Comparison with 2.138 explains the normalizations in (2.137).

One can also construct black string solutions with an asymptotically locally flat (ALF) Taub-NUT base space which has asymptotics $\mathbb{R} \times \mathrm{S}^{1} \times \mathrm{S}^{1} \times \mathbb{R}^{3}$. Since they will be the main topic of chapter 3 we present their explicit form in section 3.3 .2 . In chapters 4 and 5 we will discuss solutions with the base space given by a general ALE or ALF space. 
Uplift black ring. A five-dimensional black ring solution 91,118, 124 is asymptotically flat, has a regular horizon with topology $\mathrm{S}^{1} \times \mathrm{S}^{2}$ and near-horizon geometry $\mathrm{AdS}_{3} \times \mathrm{S}^{2}$. We will show that the $6 \mathrm{~d}$ uplift has horizon $\mathrm{S}^{1} \times \mathrm{S}^{3}$ and near-horizon geometry $\mathrm{AdS}_{3} \times \mathrm{S}^{3}$, and is thus consistent with the classification we stated at the beginning of this section. A more general discussion of uplifts of black rings in connection to supertubes was given in 125. Essentially, our discussion below is a particular and simple case of theirs, so we will be rather brief here and only focus on the near-horizon geometry.

To be specific, we take the black ring solution from [124] written in certain coordinates $v, r, \psi^{\prime}, \phi^{\prime}, \theta$ and $\chi$ and in which the near-horizon limit is taken by redefining $r=\epsilon L \tilde{r} / R, v=\tilde{v} / \epsilon$ (where $L$ and $R$ are certain constants) and sending $\epsilon \rightarrow 0$. In this limit, the metric becomes

$$
\mathrm{d} s_{5}^{2}=2 \mathrm{~d} \tilde{v} \mathrm{~d} \tilde{r}+\frac{4 L}{q} \tilde{r} \mathrm{~d} \tilde{v} \mathrm{~d} \psi^{\prime}+L^{2} \mathrm{~d} \psi^{\prime 2}+\frac{q^{2}}{4}\left(\mathrm{~d} \theta^{2}+\sin ^{2}(\theta) \mathrm{d} \chi^{2}\right)
$$

where $q$ is another constant. In the same limit, the vector field (in the conventions of section 2.4.3 is given by

$$
A=\frac{1}{2 q}\left[3 Q-q^{2}\right] \frac{C_{1}}{r} \mathrm{~d} r-\frac{q}{2} \cos (\theta) \mathrm{d} \chi,
$$

where $Q, C_{1}$ are other constants and where we have added the exact form

$$
\frac{1}{2 q}\left(3 Q-3 q^{2}\right) \mathrm{d} \psi^{\prime}+\frac{q}{2} \mathrm{~d} \phi^{\prime}+\frac{q}{2} \mathrm{~d} \psi^{\prime}
$$

to the expression in 124 . From section 2.4.3 we see that the metric of the sixdimensional uplift of a solution in the timelike class of minimal five-dimensional supergravity is given by

$$
\mathrm{d} \hat{s}_{6}^{2}=(\mathrm{d} u+A)^{2}+\mathrm{d} s_{5}^{2} .
$$

Redefining $\mathrm{d} u=-\frac{q}{2} \mathrm{~d} u^{\prime}-\frac{1}{2 q}\left[3 Q-q^{2}\right] \frac{C_{1}}{r} \mathrm{~d} r$ (this can be done in the full solution), the metric becomes

$\mathrm{d} \hat{s}_{6}^{2}=\frac{q^{2}}{4}\left(\mathrm{~d} u^{\prime}+\cos (\theta) \mathrm{d} \chi\right)^{2}+2 \mathrm{~d} \tilde{v} \mathrm{~d} \tilde{r}+\frac{4 L}{q} \tilde{r} \mathrm{~d} \tilde{v} \mathrm{~d} \psi^{\prime}+L^{2} \mathrm{~d} \psi^{\prime 2}+\frac{q^{2}}{4}\left(\mathrm{~d} \theta^{2}+\sin ^{2}(\theta) \mathrm{d} \chi^{2}\right)$.

The $u^{\prime}, \theta$ and $\chi$ part form the round metric on $\mathrm{S}^{3}$, where we need $0 \leq u^{\prime}<4 \pi$ to make it regular. The near-horizon geometry of the uplift of the black ring is thus $\mathrm{AdS}_{3} \times \mathrm{S}^{3}$. 
Black lens. In minimal five-dimensional supergravity one also has solutions that have a horizon with lens space topology $L(m, 1)=\mathrm{S}^{3} / \mathbb{Z}_{m}$ and are asymptotically flat 92,94 . In section 2.4.3 is described how such solutions can be uplifted to six dimensions. They will fall in the class with a Gibbons-Hawking base space and have harmonic functions of the form 2.122 with $m$ centers. Their near-horizon geometry will locally be given by $\mathrm{AdS}_{3} \times \mathrm{S}^{3} / \mathbb{Z}_{m}$ and their asymptotics will be $\mathbb{R} \times \mathrm{S}^{1} \times \mathbb{R}^{4}$. Note that solutions with the same near-horizon geometry but different asymptotics are given by the previously described black string with asymptotics $\mathbb{R} \times \mathrm{S}^{1} \times \mathbb{R}^{4} / \mathbb{Z}_{m}$ and by a black string with a Taub-NUT space as base space which will have asymptotics $\mathbb{R} \times \mathrm{S}^{1} \times \mathrm{S}^{1} \times \mathbb{R}^{3}$.

\subsection{6 pp-waves}

A pp-wave is characterized by the existence of a covariant constant null vector field. This vector field is necessarily a Killing vector field. Requiring the null Killing vector field $\partial_{v}$ of the general solution to be covariantly constant implies that

$$
\mathrm{d}\left[H^{-1}(\mathrm{~d} u+\beta)\right]=0
$$

which is equivalent to $\mathcal{D} \beta=0$ and $H^{-1}(\mathcal{D} H)=-\dot{\beta}$.

A first class of pp-waves is given by non-twisting solutions of section 2.4 .1 with $H=H(u)$. It follows from the construction of the coordinates in section 2.3.1 that in this case we may choose $H=1$ by redefining $u$ in 2.30 , such that the solution becomes

$$
\begin{aligned}
\mathrm{d} \hat{s}_{6}^{2} & =2 \mathrm{~d} u\left(\mathrm{~d} v+\omega+\frac{1}{2} \mathcal{F} \mathrm{d} u\right)+\mathrm{d} s_{4}^{2}, \\
\hat{G}^{\alpha} & =-e^{+} \wedge \mathrm{d} u \wedge \tilde{\mathrm{d}} \hat{\jmath}^{\alpha}+\mathrm{d} u \wedge\left[\hat{\jmath}^{\alpha} \psi-\hat{\jmath}^{\alpha}(\tilde{\mathrm{d}} \omega)^{-}+H_{\mathrm{SD}}^{\alpha}\right]+*_{4} \tilde{\mathrm{d}} \hat{\jmath}^{\alpha} .
\end{aligned}
$$

The flat base space solution derived in section 2.4.1 is an example of a pp-wave when we take the functions $P^{\alpha}$ and $Q^{\alpha}$ such that $P_{\alpha} P^{\alpha}=1, P_{\alpha} Q^{\alpha}=0$ and $Q_{\alpha} Q^{\alpha}=0$. This is only possible when $Q^{\alpha}=0$. Even with all these extra conditions, the tensor branch of the theory provides a generalization of the solution in 77. since in general the scalars are still $u$-dependent and the two-forms $H_{\mathrm{SD}}^{\alpha}$ are non-vanishing. To simplify a bit more we choose $\eta=1, W_{1}=0$ and take $C_{3}$ such that $\Omega_{\alpha \beta} C_{3}^{\alpha} P^{\beta}=2$. Transforming to Cartesian coordinates (see [102]) we find that

$$
\omega=\frac{1}{4} \Omega_{\alpha \beta} C_{3}^{\alpha} P^{\beta} r^{2} \sigma_{R}^{3}=x^{1} \mathrm{~d} x^{2}-x^{2} \mathrm{~d} x^{1}+x^{3} \mathrm{~d} x^{4}-x^{4} \mathrm{~d} x^{3} .
$$


For this solution also $(\tilde{\mathrm{d}} \omega)^{-}=0$. Performing now the coordinate transformation

$$
\begin{array}{ll}
x^{1}=\sin (u) y^{1}-\cos (u) y^{2}, & x^{2}=\cos (u) y^{1}+\sin (u) y^{2}, \\
x^{3}=\cos (u) y^{3}+\sin (u) y^{4}, & x^{4}=-\sin (u) y^{3}+\cos (u) y^{4},
\end{array}
$$

we obtain the plane wave solution

$$
\begin{aligned}
\mathrm{d} \hat{s}_{6}^{2} & =2 \mathrm{~d} u \mathrm{~d} v+\left(\mathcal{F}-\delta_{m n} y^{m} y^{n}\right) \mathrm{d} u^{2}+\delta_{m n} \mathrm{~d} y^{m} \mathrm{~d} y^{n}, \\
\hat{G}^{\alpha} & =\left(C_{3}^{\alpha}-2 P^{\alpha}\right) \mathrm{d} u \wedge\left[\mathrm{d} y^{1} \wedge \mathrm{d} y^{2}+\mathrm{d} y^{3} \wedge \mathrm{d} y^{4}\right], \\
\hat{\jmath}^{\alpha} & =P^{\alpha}(u) .
\end{aligned}
$$

A second class of pp-waves are the solutions in which $\hat{G}^{\alpha}$ vanish. A subset of these solutions is given by the vacuum solutions in which also the (physical) scalars vanish. From 2.41 we find that $\hat{G}^{\alpha}=0$ is equivalent to

$$
H^{-1} \mathcal{D} H=-\dot{\beta}, \quad \mathcal{D} \beta=0, \quad \mathcal{D} \hat{\jmath}^{\alpha}=0, \quad H \psi=(\mathcal{D} \omega)^{-}, \quad H_{\mathrm{SD}}^{\alpha}=0
$$

The first and second conditions define a pp-wave. The set of equations 2.151 will be hard to solve without extra assumptions. Of course, one can again look at the subclasses of non-twisting and $u$-independent solutions. As an example of a non-twisting solution that falls in this class, we can take 2.150 with $C_{3}^{\alpha}=2 P^{\alpha}$. In this case the solution simplifies to

$$
\begin{aligned}
\mathrm{d} \hat{s}_{6}^{2} & =2 \mathrm{~d} u \mathrm{~d} v+\left(C_{5}(u)-\frac{1}{2} C_{4}(u) \frac{1}{r^{2}}+\frac{1}{4} \Omega_{\alpha \beta} \dot{P}^{\alpha} \dot{P}^{\beta} r^{2}\right) \mathrm{d} u^{2}+\delta_{m n} \mathrm{~d} y^{m} \mathrm{~d} y^{n} \\
\hat{G}^{\alpha} & =0 \\
\hat{\jmath}^{\alpha} & =P^{\alpha}(u)
\end{aligned}
$$

where we still have the condition $P_{\alpha} P^{\alpha}=1$.

One last example we consider is not a proper pp-wave, but it is a black string with traveling waves that carry momentum along the string [126,127]. This solution falls into the non-twisting class with flat base space (section 2.4.1). Taking $W_{1}=C_{3}^{\alpha}=C_{5}=0$ and $P^{\alpha}$ and $Q^{\alpha}$ constant such that $\Omega_{\alpha \beta} P^{\alpha} P^{\beta}=1$, we find the solution

$$
\begin{aligned}
\mathrm{d} \hat{s}_{6}^{2} & =2 H^{-1} \mathrm{~d} u\left(\mathrm{~d} v-\frac{1}{4} C_{4}(u) \frac{1}{r^{2}} \mathrm{~d} u\right)+H d s_{4}^{2}, \\
\hat{G}^{\alpha} & =-\mathrm{d} v \wedge \mathrm{d} u \wedge \tilde{\mathrm{d}}\left(H^{-1} \hat{\jmath}^{\alpha}\right)+*_{4} \tilde{\mathrm{d}}\left(\hat{\jmath}^{\alpha} H\right), \\
\hat{\jmath}^{\alpha} H & =P^{\alpha}+\frac{Q^{\alpha}}{r^{2}} .
\end{aligned}
$$


In the limit $r \rightarrow \infty$ this solution is the same as 2.152 with $C_{5}=\dot{P}^{\alpha}=0$, but note that the full solution is very different, mainly because we now have non-vanishing three-forms to support the black string and also the scalars depend on the base space instead of on $u$. For a further discussion of this kind of geometry, see e.g. the original references 126, 127. The horizon of these solutions become singular however, as discussed e.g. in 128 130]. Perhaps a more interesting class of solutions are the traveling wave deformations of smooth horizonless solutions, such as discussed e.g. in 115. It could be interesting to extend the analysis of 115 to the present setup where more tensor multiplets are present.

\subsection{Concluding remarks}

In this chapter we derived and analyzed the general local form of supersymmetric solutions of $\mathcal{N}=(1,0)$ supergravity coupled to tensor multiplets and studied examples of BPS black strings and pp-waves with non-trivial scalar profiles. There are many interesting extensions and generalizations, such as the study of bound states of black strings, and the construction of new microstate geometries and their dual CFT states.

In chapters 35 we will study further the embedding in F-theory and type IIB string theory of some of the black string solutions constructed in this chapter. In particular we compute the central charges and levels of the dual $\mathcal{N}=(0,4)$ SCFTs from macroscopics and match them to the microscopic values.

\section{Appendices}

\section{A Integrability conditions}

In this appendix we derive which equations of motion are implied by the integrability conditions of the theory. Denote the scalar equations of motion by $(E \hat{\jmath})^{M}=0$, the three-form equations of motion by $(E \hat{G})_{\alpha}^{\mu \nu}=\hat{\nabla}_{\lambda}\left(g_{\alpha \beta} \hat{G}^{\beta} \lambda \mu \nu\right)=0$ and the Einstein equation by $E_{\mu \nu}=0$. Contracting the integrability of the gravitino Killing spinor equation with $\gamma^{\nu}$ yields 8

$$
\gamma^{\nu}\left[\mathcal{D}_{\mu}, \mathcal{D}_{\nu}\right] \epsilon=E_{\mu \nu} \gamma^{\nu} \epsilon+\frac{1}{8} \hat{\jmath}^{\alpha}(E \hat{G})_{\alpha \rho \sigma} \hat{g}_{\mu \nu} \gamma^{\nu \rho \sigma} \epsilon-\frac{1}{4} \hat{\jmath}^{\alpha}(E \hat{G})_{\alpha \mu \nu} \gamma^{\nu} \epsilon=0
$$

${ }^{8}$ This condition is derived in the PhD thesis of Mehmet Akyol (Kings college). 
Assuming the three-form equations of motion it follows that

$$
E_{\mu \nu} \gamma^{\nu} \epsilon=0
$$

In the null-basis $\gamma_{+} \epsilon=0$, thus we observe that 2.155 implies that

$$
E_{\mu+}=E_{\mu 1}=E_{\mu 2}=E_{\mu 3}=E_{\mu 4}=0
$$

Hence, only the $E_{--}$component is not determined by the integrability conditions.

The integrability of the tensorini Killing spinor equation contracted with $\gamma^{\mu}$ and expressed in the equations of motion yields 9

$$
\gamma^{\mu}\left[\mathcal{D}_{\mu}, T_{\nu}^{M} \gamma^{\nu}-\frac{1}{12} H_{\nu \rho \sigma}^{M} \gamma^{\nu \rho \sigma}\right] \epsilon=(E \hat{\jmath})^{M} \epsilon+\frac{1}{2} x_{\alpha}^{M}(E \hat{G})_{\mu \nu}^{\alpha} \gamma^{\mu \nu} \epsilon=0
$$

Assuming the three-form equations of motion, it follows that 2.157 is equivalent to the scalar equations of motion.

\section{B Spin connection}

In this appendix, the components $i, j, \ldots$ will refer to the part of the six-dimensional vielbein $\hat{e}^{i}$, unless they are components of base space objects $\tilde{\omega}$ and $\tilde{e}^{i}$. Using metric compatibility, so anti-symmetry of the connection

$$
\begin{aligned}
& \hat{\omega}_{i j}=-\hat{\omega}_{j i}, \quad \hat{\omega}_{+i}=-\hat{\omega}_{i+}, \quad \hat{\omega}_{-i}=-\hat{\omega}_{i-}, \\
& \hat{\omega}_{++}=0, \quad \hat{\omega}_{--}=0, \quad \hat{\omega}_{+-}=-\hat{\omega}_{-+} \text {, }
\end{aligned}
$$

\footnotetext{
${ }^{9}$ This condition is derived in the PhD thesis of Mehmet Akyol (Kings college), although there the wrong scalar equation of motion is used.
} 
and vanishing torsion, a straightforward calculation yields that the spin connection is given by

$$
\begin{aligned}
\hat{\omega}^{+}{ }_{i}= & \frac{1}{2}\left(\mathcal{D} \omega+\frac{1}{2} \mathcal{F} \mathcal{D} \beta\right)_{i j} \hat{e}^{j}-\frac{1}{2} \partial_{u}\left(H h_{m n}\right) \tilde{e}_{i}^{m} \tilde{e}_{j}^{n} \hat{e}^{j}-H\left(\dot{\omega}+\frac{1}{2} \mathcal{F} \dot{\beta}-\frac{1}{2} \mathcal{D} \mathcal{F}\right)_{i} e^{-} \\
& -\frac{1}{2}\left(H^{-1} \mathcal{D} H+\dot{\beta}\right)_{i} e^{+}, \\
\hat{\omega}^{-}{ }_{i}= & \frac{1}{2} H^{-1}(\mathcal{D} \beta)_{i j} \hat{e}^{j}-\frac{1}{2}\left(H^{-1} \mathcal{D} H+\dot{\beta}\right)_{i} e^{-}, \\
\hat{\omega}^{+}{ }_{+}= & -\frac{1}{2}\left(H^{-1} \mathcal{D} H+\dot{\beta}\right)_{i} \hat{e}^{i}, \\
\hat{\omega}^{-}{ }_{-}= & \frac{1}{2}\left(H^{-1} \mathcal{D} H+\dot{\beta}\right)_{i} \hat{e}^{i}, \\
\hat{\omega}^{i}{ }_{j}= & \tilde{\omega}^{i}{ }_{j}+\frac{1}{2} H^{-1}(\mathcal{D} H)_{j} \hat{e}^{i}-\frac{1}{2} H^{-1}(\mathcal{D} H)^{i} \delta_{j k} \hat{e}^{k}+\frac{1}{2} H^{1 / 2}\left(\beta \wedge \dot{\tilde{e}}^{i}\right)_{k j} \hat{e}^{k} \\
& +\frac{1}{2} H^{1 / 2}\left(\beta \wedge \dot{\tilde{e}}_{k}\right)^{i}{ }_{j} \hat{e}^{k}+\frac{1}{2} H^{1 / 2}\left(\beta \wedge \dot{\tilde{e}}_{j}\right)^{i}{ }_{k} \hat{e}^{k}-\frac{1}{2} H^{-1}(\mathcal{D} \beta)^{i}{ }_{j} e^{+} \\
& -\frac{1}{2}\left(\mathcal{D} \omega+\frac{1}{2} \mathcal{F} \mathcal{D} \beta\right)^{i}{ }_{j} e^{-}-H{ }^{k}\left(\partial_{u} \tilde{e}_{m}^{[i}\right)^{i} \tilde{e}_{j]}^{m} e^{-} \cdot
\end{aligned}
$$

\section{C $\hat{\boldsymbol{R}}_{--}$component of the Ricci tensor}

In this appendix, the components $i, j, \ldots$ will at first refer to the part of the six-dimensional vielbein $\hat{e}^{i}$, unless they are components of base space objects $\tilde{\omega}$ and $\tilde{e}^{i}$ or indicated as $\tilde{i}$. We would like to calculate the -- component of the Ricci tensor:

$$
\hat{R}_{--}=\hat{R}_{-+-}^{+}+\hat{R}_{-i-}^{i} .
$$

We calculate the curvature two-form via the spin-connection:

$$
\begin{aligned}
& \hat{R}^{i}{ }_{-}=\mathrm{d} \hat{\omega}^{i}{ }_{-}+\hat{\omega}^{i}{ }_{j} \wedge \hat{\omega}^{j}{ }_{-}+\hat{\omega}^{i}{ }_{-} \wedge \hat{\omega}^{-}{ }_{-}, \\
& \hat{R}^{+}{ }_{-}=\mathrm{d} \hat{\omega}^{+}{ }_{-}+\hat{\omega}^{+}{ }_{i} \wedge \hat{\omega}^{i}{ }_{-},
\end{aligned}
$$


where we will only keep the $\hat{R}_{-j-}^{i}$ and $\hat{R}_{-+-}^{+}$components. A straightforward (but lengthy) calculation yields

$$
\begin{aligned}
\hat{R}_{-}^{+}= & 0 \\
\hat{R}_{-}^{i} \rightarrow & \left\{H \partial_{u}\left[\frac{1}{2}\left(\mathcal{D} \omega+\frac{1}{2} \mathcal{F} \mathcal{D} \beta\right)_{j}^{i}-\frac{1}{2} \partial_{u}\left(H h_{m n}\right) \tilde{e}^{m i} \tilde{e}_{j}^{n}\right]\right. \\
& +\left[\frac{1}{2}\left(\mathcal{D} \omega+\frac{1}{2} \mathcal{F} \mathcal{D} \beta\right)_{k}^{i}-\frac{1}{2} \partial_{u}\left(H h_{m n}\right) \tilde{e}^{m i} \tilde{e}_{k}^{n}\right]\left[\frac{1}{2} \dot{H} \delta_{j}^{k}+H\left(\partial_{u} \tilde{e}_{o}^{k}\right) \tilde{e}_{j}^{o}\right] \\
& +\frac{1}{2} H^{-1}\left(\partial_{\tilde{j}} H\right)\left(\dot{\omega}+\frac{1}{2} \mathcal{F} \dot{\beta}-\frac{1}{2} \mathcal{D} \mathcal{F}\right)^{\tilde{i}}+\tilde{\nabla}_{\tilde{j}}\left[\left(\dot{\omega}+\frac{1}{2} \mathcal{F} \dot{\beta}-\frac{1}{2} \mathcal{D} \mathcal{F}\right)^{\tilde{i}}\right] \\
& -\partial_{u}\left[H\left(\dot{\omega}+\frac{1}{2} \mathcal{F} \dot{\beta}-\frac{1}{2} \mathcal{D} \mathcal{F}\right)^{i}\right] \beta_{j}-\frac{3}{2} H\left(\dot{\omega}+\frac{1}{2} \mathcal{F} \dot{\beta}-\frac{1}{2} \mathcal{D} \mathcal{F}\right)^{i} \\
& \times\left(H{ }^{-1} \mathcal{D} H+\dot{\beta}\right)_{j}-\frac{1}{2} H\left(H^{-1} \mathcal{D} H+\dot{\beta}\right)^{i}\left(\dot{\omega}+\frac{1}{2} \mathcal{F} \dot{\beta}-\frac{1}{2} \mathcal{D} \mathcal{F}\right)_{j} \\
& +H\left(\dot{\omega}+\frac{1}{2} \mathcal{F} \dot{\beta}-\frac{1}{2} \mathcal{D} \mathcal{F}\right)^{k}\left[\frac{1}{2} H^{-1}(\mathcal{D} H)_{k} \delta_{j}^{i}-\frac{1}{2} H^{-1}(\mathcal{D} H)^{i} \delta_{k j}\right. \\
& \left.+\frac{1}{2} H^{1 / 2}\left(\beta \wedge \dot{\tilde{e}}^{i}\right)_{j k}+\frac{1}{2} H^{1 / 2}\left(\beta \wedge \dot{\tilde{e}}_{j}\right)_{k}{ }_{k}+\frac{1}{2} H^{1 / 2}\left(\beta \wedge \dot{\tilde{e}}_{k}\right)_{j}^{i}{ }_{j}\right] \\
& -\left[\frac{1}{2}\left(\mathcal{D} \omega+\frac{1}{2} \mathcal{F} \mathcal{D} \beta\right)_{k}^{i}+H^{i}\left(\partial_{u} e_{m}^{[i}\right)_{k]}^{m}\right] \\
& \left.\times\left[\frac{1}{2}\left(\mathcal{D} \omega+\frac{1}{2} \mathcal{F} \mathcal{D} \beta\right)^{k}{ }_{j}-\frac{1}{2} \partial_{u}\left(H h_{m n}\right)_{\tilde{e}}^{m k} \tilde{e}_{j}^{n}\right]\right\} \hat{e}^{j} \wedge e^{-} .
\end{aligned}
$$

Taking the $\hat{R}_{-i-}^{i}$ components, summing over $i$ and rewriting everything in components with respect to the vielbein $\tilde{e}^{i}$ yields

$$
\begin{aligned}
\hat{R}_{--}= & *_{4} \mathcal{D} *_{4}\left(\dot{\omega}+\frac{1}{2} \mathcal{F} \dot{\beta}-\frac{1}{2} \mathcal{D} \mathcal{F}\right)-2\left(\dot{\omega}+\frac{1}{2} \mathcal{F} \dot{\beta}-\frac{1}{2} \mathcal{D} \mathcal{F}\right)^{m} \partial_{u}\left(\beta_{m}\right) \\
& +\frac{1}{4} H^{-2}\left(\mathcal{D} \omega+\frac{1}{2} \mathcal{F} \mathcal{D} \beta\right)_{i k}\left(\mathcal{D} \omega+\frac{1}{2} \mathcal{F} \mathcal{D} \beta\right)^{i k} \\
& -\frac{1}{2} H h^{m n} \partial_{u}^{2}\left(H h_{m n}\right)-\frac{1}{4} \partial_{u}\left(H h_{m n}\right) \partial_{u}\left(H h^{m n}\right) .
\end{aligned}
$$

\section{D Single string solution}

Taking a single string at the origin, we still have to determine the one-forms $\vec{\chi}, \vec{\beta}$ and $\vec{\omega}$. Solving the equations for $\vec{\chi}, \vec{\beta}$ and $\vec{\omega}$, and assuming the Bubble equations, we find that

$$
\chi_{a} d x^{a}=m \cos (\theta) d \phi, \quad \beta_{a} d x^{a}=-q \cos (\theta) d \phi, \quad \omega_{a} d x^{a}=0 .
$$

We now require the metric to asymptote to $\mathbb{R} \times \mathrm{S}_{u}^{1} \times \mathbb{R}^{4} / \mathbb{Z}_{m}$. This implies that 
$m_{\infty}=0$, the functions $H, \mathcal{F} \rightarrow 1$ and the one-forms $\omega, \beta \rightarrow 0$. The limit

$$
\lim _{r \rightarrow \infty} \mathcal{F}=-n_{\infty}+\frac{r}{m} \Omega_{\alpha \beta} p_{\infty}^{\alpha} p_{\infty}^{\beta}+2 \frac{1}{m} \Omega_{\alpha \beta} p_{\infty}^{\alpha} p^{\beta}=1
$$

implies that $p_{\infty}^{\alpha}=0$ and $n_{\infty}=-1$. The limit of the one-form $\beta \rightarrow 0$ implies that $q_{\infty}=0$ such that $\beta \rightarrow \frac{q}{m} d \psi$. This can be absorbed by the coordinate redefinition $\mathrm{d} u \rightarrow \mathrm{d} u-\frac{q}{m} \mathrm{~d} \psi$. For the single string, $u$ is periodic, hence we need that

$$
\frac{4 \pi q}{\operatorname{lm}} \in \mathbb{Z},
$$

where $l$ is the length of the circle, for this to be well-defined. The limit

$$
\lim _{r \rightarrow \infty} H=\sqrt{\Omega_{\alpha \beta} \mu_{\infty}^{\alpha} \mu_{\infty}^{\alpha}}=1
$$

then implies that

$$
\Omega_{\alpha \beta} \mu_{\infty}^{\alpha} \mu_{\infty}^{\beta}=1
$$

Lastly, the limit of the one-form $\omega \rightarrow 0$ implies that

$$
\lim _{r \rightarrow 0} \omega_{0}=j_{\infty}+\frac{1}{m} \Omega_{\alpha \beta} \mu_{\infty}^{\alpha} p^{\beta}-\frac{1}{2} \frac{q}{m}=0
$$

such that

$$
j_{\infty}=\frac{1}{2} \frac{q}{m}-\frac{1}{m} \Omega_{\alpha \beta} \mu_{\infty}^{\alpha} p^{\beta} .
$$

Hence, to get the correct asymptotics, we need

$$
\Gamma_{\infty}=\left(\mu_{\infty}^{\alpha}, 0,0,0,-1, \frac{1}{2} \frac{q}{m}-\frac{1}{m} \Omega_{\alpha \beta} \mu_{\infty}^{\alpha} p^{\beta}\right)
$$

subject to 2.168). With these values for $\Gamma_{\infty}$, the Bubble equations are automatically satisfied. 



\section{Black strings in F-theory with Taub-NUT space transversely}

In the preceding chapter we have derived a general local form for supersymmetric solutions of $6 \mathrm{~d} \mathcal{N}=(1,0)$ supergravity coupled to an arbitrary number of tensor multiplets. We also considered some special cases in which the resulting equations could be solved explicitly. In particular we derived black string solutions. In this chapter and the next two chapters we will study the embedding of some of these black strings in F-theory and type IIB string theory. As we briefly discussed in section 1.2.5 compactifying F-theory on an elliptically fibered Calabi-Yau threefold yields an effective $6 \mathrm{~d} \mathcal{N}=(1,0)$ supergravity theory. Black string solutions of this theory can be embedded in F-theory as D3-branes wrapping a curve in the base of the fibration. In this chapter, which is based on [2], we will be particularly interested in black string solutions that can be compactified to four-dimensional black holes. As we have discussed in section 1.3.2 the entropy of these objects is determined via the Cardy formula in terms of the central charges and levels of an $\mathcal{N}=(0,4)$ superconformal field theory (SCFT). This SCFT is the IR-limit of the compactified worldvolume theory of the D3-branes on the curve. The goal of this chapter is to determine these central charges and levels using the macroscopic supergravity theory with the techniques outlined in section 1.4.1. We will thus determine the entropy of four-dimensional black holes in F-theory.

\subsection{Introduction}

In the early days of F-theory the microscopic entropy of a D3-brane in an asymptotic geometry $\mathbb{R}^{1,4} \times \mathrm{S}^{1} \times \mathrm{CY}_{3}$ was computed exploiting its dual M-theory formulation. The D3-brane is wrapped on $\mathrm{S}^{1} \times C$, where $C$ is a curve in the base $B$ of an elliptically fibered Calabi-Yau threefold $\mathrm{CY}_{3}$ and corresponds to a non-spinning black hole in five dimensions. The microscopic entropy was then successfully matched to its macroscopic counterpart [44]. Some years later, this setup was generalized to an asymptotic geometry $\mathbb{R} \times \mathrm{S}^{1} \times \mathrm{TN}_{m} \times \mathrm{CY}_{3}[75$ which corresponds to macroscopic four-dimensional black holes. The microscopic analysis was carried out by mapping 
the F-theory setup to the MSW setting [45]. Studying gravitational aspects in F-theory attracted renewed interest recently using diverse approaches. In 76 the authors extended the study of [44] to the case of five-dimensional spinning black holes that previously only had been studied in compactifications of type II strings on $\mathrm{T}^{5}$ and $\mathrm{K} 3 \times \mathrm{S}^{1}[43]$. On the CFT side the main difference between [44] and $\left[76\right.$ is the identification of an $\mathfrak{s u}(2)_{L}$ current algebra in spite of the absence of supersymmetry in the left-moving sector. Most recently, supersymmetric $\mathrm{AdS}_{3}$ solutions of type IIB supergravity with varying axio-dilaton profile and five-form flux dual to $\mathcal{N}=(0, n), n=2,4$ SCFTs were analyzed in 131, 132. The $\mathrm{AdS}_{3}$ solutions dual to $\mathcal{N}=(0,4)$ SCFTs can be interpreted as near-horizon geometries of six-dimensional strings from wrapped D3-branes, as described above.

In this chapter we derive characteristic data of the SCFT corresponding to the D3-brane wrapped inside the $\mathbb{R} \times \mathrm{S}^{1} \times \mathrm{TN}_{m} \times \mathrm{CY}_{3}$ geometry 75 from macroscopic considerations. The two-dimensional SCFT has $\mathcal{N}=(0,4)$ supersymmetry and leftand right-moving central charges $c_{L, R}$, as well as left- and right-moving current algebras $U(1)_{L} \times S U(2)_{R}$ with levels $k_{L, R}$. The consideration of this setting is strongly motivated by the $4 \mathrm{D} / 5 \mathrm{D}$ black hole correspondence 133 134. Making the radius of the circle $\mathrm{S}^{1}$ very small, we perform a T-duality along $\mathrm{S}^{1}$ to obtain a type IIA setting that then lifts to an M-theory background $\mathbb{R} \times \mathrm{TN}_{m} \times \mathrm{CY}_{3}$. Under this duality the wrapped D3-brane turns into an M2-brane wrapping $C$. Momentum $n$ along $\mathrm{S}^{1}$ corresponds to bound states of M2-branes wrapping a curve in the class $C+n \mathbb{E}_{\tau}$, where $\mathbb{E}_{\tau}$ is the elliptic fiber of the $\mathrm{CY}_{3}$. After compactification on $\mathrm{CY}_{3}$ one obtains a five-dimensional black hole with a transverse Taub-NUT spacetime. This five-dimensional black hole has an eigenvalue $J_{L}$ which corresponds to the $U(1)_{L}$ symmetry along the NUT-circle. Compactifying the M-theory setting further along the circle fiber of the Taub-NUT space results in a type IIA compactification on $\mathrm{CY}_{3}$. The M2-brane configuration gets mapped to a D6-D2-D0 system on the same Calabi-Yau threefold 17 The D6-brane has multiplicity $m$ and one has $2 J_{L}$ units of D0-brane charge. This is the four-dimensional side of the correspondence in 133,134 .

Instead of using the circle $\mathrm{S}^{1}$ wrapped by the D3-brane to go to five dimensions, we can also reduce along the NUT-circle, which we denote by $\tilde{\mathrm{S}}^{1}$. Performing a T-duality along $\tilde{\mathrm{S}}^{1}$, and lifting to M-theory, the D3-brane wrapping the curve $C$ turns into an M5-brane wrapping $\hat{C}=\pi^{-1}(C) \subset \mathrm{CY}_{3}$, where $\pi: \mathrm{CY}_{3} \rightarrow B$ is the projection to the base. Following the same duality the Taub-NUT space gives rise to $m$ M5-branes wrapping the base $B$ of the elliptic fibration. The two groups of

\footnotetext{
${ }^{1}$ Recall the discussion in section 1.2 .4 .2
} 
M5-branes can be combined into a single M5-brane wrapping the curve $\hat{C}+m B$ in the $\mathrm{CY}_{3}$ if the corresponding class is very ample. In summary, the two main dualities just introduced can be depicted schematically as:

$$
\begin{aligned}
& \text { F-theory on } \mathbb{R} \times \mathrm{S}^{1} \times \mathrm{TN}_{m} \times \mathrm{CY}_{3} \\
& \text { with D3-brane wrapping } \mathrm{S}^{1} \times C
\end{aligned}
$$

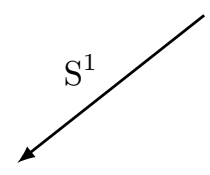

M-theory on $\mathbb{R} \times \mathrm{TN}_{m} \times \mathrm{CY}_{3}$

with M2's wrapping $C+n \mathbb{E}_{\tau}$

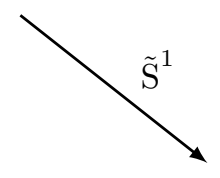

M-theory on $\mathbb{R} \times \mathrm{S}^{1} \times \mathbb{R}^{3} \times \mathrm{CY}_{3}$

with M5 wrapping $\mathrm{S}^{1} \times(\hat{C}+m B)$

If we take both the NUT-circle and the circle wrapped by the D3-brane to be small, we obtain an effectively four-dimensional black hole. We therefore obtain an F-theory description of a four-dimensional black hole. The central charges and levels we determine in this chapter then give the black hole entropy via the Cardy formula 1.94 $2^{2}$

We use the six-dimensional effective $\mathcal{N}=(1,0)$ supergravity action of F-theory compactified on an elliptically fibered Calabi-Yau threefold derived in [37, 135] to determine the contributions from classical six-dimensional supergravity to the central charges and levels using techniques of [55,58, 136] which we discussed in section 1.4.1. Concretely, we dimensionally reduce the six-dimensional effective action to three dimensions and read off the sought-after quantities from the coefficients of Chern-Simons terms. It turns out that in order to fully reproduce the microscopic quantities one also has to include one-loop Chern-Simons terms in three dimensions. These one-loop induced terms arise from integrating out massive Kaluza-Klein modes. This interplay between classical and quantum contributions to complete $\mathrm{M} / \mathrm{F}$-theory duality in this case is in fact not unexpected. Including one-loop corrections was already crucial for the matching of the five-dimensional M-theory effective action on $\mathrm{CY}_{3}$ and its dual six-dimensional F-theory action 37,119$] 3$ We furthermore utilize the procedure to do the dimensional reduction 'at asymptotic

${ }^{2}$ As discussed in section 1.4.1 the central charges and levels we compute actually determine an index. However, the part we determine is equal to the entropy.

${ }^{3}$ Recently corrections to the entropy due to one-loop Chern-Simons terms in the effective action were also computed in Scherk-Schwarz reductions of the six-dimensional D1-D5 system on a circle 137 . 
infinity' put forward in 60 and discussed in section 1.4.1 instead of doing it in the near-horizon geometry, as may be the more intuitive approach in view of the standard AdS/CFT dictionary. As a last ingredient for the comparison with the microscopic charges we also take into account a shift in the charges stemming from a non-vanishing higher derivative term on Taub-NUT space in the six-dimensional effective action. The latter two points demonstrate that the full geometry outside the horizon is important for the matching with microscopics.

In section 3.2 we start with a more extensive description of the setting we are working in. In this section we will also state the microscopic quantities that we want to reproduce from supergravity. We then proceed by calculating the classical and quantum contributions to the central charges and levels in sections 3.3 and 3.4 respectively. Subsequently we summarize and comment on the $4 \mathrm{D} / 5 \mathrm{D}$ correspondence in section 3.5 Finally, we discuss our results in section 3.6

\subsection{Microscopics}

As already stated in the introduction of this chapter, we consider an F-theory background $\mathbb{R} \times \mathrm{S}^{1} \times \mathrm{TN}_{m} \times \mathrm{CY}_{3}$, where we have a D3-brane wrapping $\mathrm{S}^{1} \times C$ with $C \subset B$ a curve in the elliptically fibered Calabi-Yau threefold $\pi: \mathrm{CY}_{3} \rightarrow B$. For simplicity we only consider threefolds with mild fiber degenerations which render the total elliptic fibration smooth. Using a basis $\omega_{\alpha}$ of $H^{1,1}(B)$ we can expand the Poincaré dual of the curve and the first Chern class of the base such that we have $C=q^{\alpha} \omega_{\alpha}$ and $c_{1}(B)=c^{\alpha} \omega_{\alpha}$. The intersection numbers on the base are given by

$$
\eta_{\alpha \beta} \equiv \int_{B} \omega_{\alpha} \wedge \omega_{\beta} .
$$

Furthermore, here and in the following we make use of the notation

$$
\begin{aligned}
C \cdot C & =\int_{B} C \wedge C \equiv C^{2}, \\
c_{1}(B) \cdot C & =\int_{B} c_{1}(B) \wedge C, \\
c_{1}(B) \cdot c_{1}(B) & =\int_{B} c_{1}(B) \wedge c_{1}(B) \equiv c_{1}(B)^{2} .
\end{aligned}
$$

Microscopically the central charges corresponding to this setting were derived by considering the dual system in M-theory 75 . As already described in the introduction of this chapter one can start from type IIB, T-dualize along the NUT-circle and then lift the system to M-theory. Performing a T-duality along the 
NUT-circle $\widetilde{\mathrm{S}}^{1}$ we end up with a D4-brane wrapping $\mathrm{S}^{1} \times \tilde{\mathrm{S}}^{1} \times C$ and $m$ NS5-branes wrapping $B$. These type IIA objects lift in M-theory to an M5-brane wrapping $\hat{C}$ and $m$ M5-branes wrapping $B$. If the class of the curve $\hat{C}+m B$ is very ample the two M5-brane groups can be combined into a single M5-brane wrapping $\hat{C}+m B$. We therefore assume that $q^{\alpha}>0$ and $q^{\alpha} \gg m c^{\alpha}, \forall \alpha=1, \ldots, h^{1,1}(B)$.

This system falls in the class of settings studied by MSW 45. such that the central charges and right level are given by ${ }^{4}$

$$
\begin{aligned}
& c_{L}=3 m C^{2}-3 m^{2} c_{1}(B) \cdot C+m^{3} c_{1}(B)^{2}+12 c_{1}(B) \cdot C+12 m-2 m c_{1}(B)^{2}, \\
& c_{R}=6 k_{R}=3 m C^{2}-3 m^{2} c_{1}(B) \cdot C+m^{3} c_{1}(B)^{2}+6 c_{1}(B) \cdot C+6 m-m c_{1}(B)^{2},
\end{aligned}
$$

where the relation between the right central charge and level follows from supersymmetry in the right-moving sector of the SCFT.

Left level. Although not explicitly calculated, we can extract the left level $k_{L}$ from the data provided in 75$]$. The formula for the entropy of the black string in 75 reads

$$
S=2 \pi \sqrt{\frac{c_{L}}{6} \hat{m}},
$$

where

$$
\hat{m}=n+\frac{1}{12}\left(D^{00} \tilde{Q}_{0} \tilde{Q}_{0}+2 D^{0 \alpha} \tilde{Q}_{0} \tilde{Q}_{\alpha}+D^{\alpha \beta} \tilde{Q}_{\alpha} \tilde{Q}_{\beta}\right)
$$

and the matrix $D$ is given by

$$
D_{00}=\frac{1}{6} c_{1}(B) \cdot C, \quad D_{0 \alpha}=\frac{1}{6} q_{\alpha}, \quad D_{\alpha \beta}=\frac{1}{6} m \eta_{\alpha \beta}
$$

The elements $D^{A B}=D_{A B}^{-1}$ denote components of the inverse matrix with respect to the full matrix $D_{A B}$, in particular, it is not the inverse of a sub-matrix of $D$. The charge $\tilde{Q}_{0}$ contains a term $2 J_{L} / m\left[75\right.$. The $U(1)$ current $J_{L}$ belongs to an $S U(2)$ current algebra and as we have seen in section 1.3 .2 the entropy of such a CFT is given by

$$
S=2 \pi \sqrt{\frac{c_{L}}{6}\left(n-\frac{J_{L}^{2}}{k_{L}}\right)},
$$

and we can read off $k_{L}$. The level should not depend on the momentum, so we can take the limit $2 J_{L} / m \rightarrow \infty$ in 3.5 and compare the resulting expression with the

${ }^{4}$ These results follow straightforwardly from the data given in 75 using identities valid for elliptically fibered Calabi-Yau threefolds. 
spectral flow invariant $n-\frac{J_{L}^{2}}{k_{L}}$. In particular we have that

$$
\frac{1}{12} D^{00}\left(\frac{2 J_{L}}{m}\right)^{2}=-\frac{J_{L}^{2}}{k_{L}} .
$$

Calculating the inverse of $D$ yields

$$
D^{00}=\frac{6 m}{m c_{1}(B) \cdot C-C^{2}},
$$

such that we find

$$
k_{L}=\frac{1}{2} m C^{2}-\frac{1}{2} m^{2} c_{1}(B) \cdot C
$$

for the left level.

Goal of this chapter. It is the main objective of this chapter to reproduce the central charges and levels, given in $(3.3)$ and $(3.10)$, from six-dimensional $\mathcal{N}=(1,0)$ supergravity up to $\mathcal{O}(1)$ terms.

Parts of the central charges and levels have been computed in [131] from type IIB supergravity. The authors studied $\mathrm{AdS}_{3}$ solutions of type IIB supergravity with varying axio-dilaton using the spinorial geometry approach. They studied the constraints on the compact geometry arising from preserving $\mathcal{N}=(0,4)$ supersymmetry in the dual two-dimensional SCFT while preserving all $\mathrm{AdS}_{3}$ isometries. The class of ten-dimensional solutions takes the form $\operatorname{AdS}_{3} \times \mathrm{S}^{3} / \mathbb{Z}_{m} \times B$, with non-trivial five-form flux and axio-dilaton profile, where $B$ is the Kähler base of an elliptically fibered Calabi-Yau threefold. The solution can be interpreted as the near-horizon limit of $N$ D3-branes wrapping a curve $C$ in the Kähler base in the presence of D7-branes and a Taub-NUT space with NUT-charge $m$ in the four non-compact directions transverse to the D3-branes. The dual $\mathcal{N}=(0,4) \mathrm{SCFT}$ has again a $U(1)_{L} \times S U(2)_{R}$ current algebra with levels $k_{L, R}$. These levels and the central charges of the CFT were computed in the large $N$ limit and were for general $m$ found to be

$$
\begin{aligned}
c_{R}^{\mathrm{IIB}} & =6 k_{R}^{\mathrm{IIB}}=3 N^{2} m C^{2}, \\
c_{L}^{\mathrm{IIB}} & =3 N^{2} m C^{2}, \\
k_{L}^{\mathrm{IIB}} & =\text { unknown } .
\end{aligned}
$$

The subleading correction $c_{L}^{\mathrm{IIB}}-c_{R}^{\mathrm{IIB}}$ at $\mathcal{O}(N)$ was also found for general $m$, it is however expected from the dual M-theory result 3.3 that there exist additional $\mathcal{O}(N)$ contributions to the central charges and levels. The full answer for $c_{L, R}^{\mathrm{IIB}}$ 
and $k_{R}^{\mathrm{IIB}}$ including $\mathcal{O}(N)$ contributions was given for the distinguished case $m=1$, where the near-horizon geometry is $\mathrm{AdS}_{3} \times \mathrm{S}^{3} \times B$ corresponding to an unbroken $S U(2)_{L} \times S U(2)_{R}$ current algebra in the CFT.

\subsection{Macroscopic classical contributions}

In this section we use six-dimensional $(1,0)$ supergravity 109 111, 138 to compute parts of the microscopic central charges and levels (3.3) and (3.10). An F-theory compactification on a smooth elliptically fibered Calabi-Yau threefold results in a gravity multiplet, $n_{T}=h^{1,1}(B)-1$ tensor multiplets and $n_{H}=h^{2,1}\left(\mathrm{CY}_{3}\right)+1$ hypermultiplets, but no vector multiplets $33,139,140$. Recall that we restricted ourselves to smooth threefolds for simplicity, e.g to avoid charged matter. We reproduce part of the central charges and levels utilizing the approach used in 55, 58, 60, 136 and discussed in section 1.4.1, which in principle means that one has to reduce the six-dimensional action on the spherical part of the near-horizon geometry $\mathrm{AdS}_{3} \times \mathrm{S}^{3} / \mathbb{Z}_{m}$ of the black string solution. Dimensionally reducing the six-dimensional action on $\mathrm{S}^{3} / \mathbb{Z}_{m}$ one can infer the levels and central charges of the dual CFT from coefficients of Chern-Simons terms in three dimensions using the AdS/CFT dictionary. In fact we find, based on [60], that one has to do this dimensional reduction at spatial infinity of the solution to get the correct result for central charges and levels and to take into account the effect of the Taub-NUT space transverse to the string.

We first provide a few details about the six-dimensional $\mathcal{N}=(1,0)$ supergravity theory arising from F-theory compactified on a Calabi-Yau threefold, which shall be the starting point for our investigation. In the sequel we perform the dimensional reduction of the supergravity action to three dimensions, pointing out the difference between the reduction in the near-horizon geometry and the reduction at asymptotic infinity. In both cases one finds a mismatch with the microscopic prediction. The mismatch in the reduction at asymptotic infinity can be cured using one loop induced Chern-Simons terms in three dimensions. This will be the subject of section 3.4 which is one of the main results of this chapter.

\subsubsection{Six-dimensional supergravity from F-theory on $\mathrm{CY}_{3}$}

We consider the six-dimensional effective action arising from compactifying F-theory on an elliptically fibered Calabi-Yau threefold $\mathrm{CY}_{3}$. The characteristic data of the underlying $\mathcal{N}=(1,0)$ supergravity in six dimensions was determined in [37] 
by matching a generic circle reduced six-dimensional $\mathcal{N}=(1,0)$ theory with the geometric data arising from compactifying M-theory on a smooth Calabi-Yau threefold. The massless spectrum assembles itself in representations of the little group in six dimensions $S O(4) \simeq S U(2)_{1} \times S U(2)_{2}$, which are labeled by the spins $\left(j_{1}, j_{2}\right)$. We will focus on a six-dimensional theory with field content

- one gravity multiplet: $(1,1) \oplus 2\left(1, \frac{1}{2}\right) \oplus(1,0)$ i.e. one graviton, one left-handed gravitino and one self-dual rank two tensor

- $n_{T}$ tensor multiplets: $(0,1) \oplus 2\left(0, \frac{1}{2}\right) \oplus(0,0)$ i.e. one anti-self-dual rank two tensor, one right-handed tensorino and one real scalar

- $n_{H}$ uncharged hypermultiplets: $2\left(0, \frac{1}{2}\right) \oplus 4(0,0)$ i.e. one right-handed hyperino and two complex scalars.

We will furthermore assume throughout the chapter that the six-dimensional spectrum satisfies the anomaly constraint

$$
n_{H}=273-29 n_{T} .
$$

Tensor multiplets. As we have seen in section 2.2 the rank two tensors in the gravity- and tensor multiplets can be denoted by $\hat{B}^{\alpha}$ with $\alpha=1, \ldots, n_{T}+1$. The scalars in the tensor multiplets parametrize the manifold

$$
\mathcal{M}_{\text {tensor }}=\frac{S O\left(1, n_{T}\right)}{S O\left(n_{T}\right)} .
$$

We describe the scalar sector of the tensor multiplets by $n_{T}+1$ scalar fields $\hat{\jmath}^{\alpha}$ subject to the constraint

$$
\Omega_{\alpha \beta} \hat{\jmath}^{\alpha} \hat{\jmath}^{\beta}=1
$$

where $\left(\Omega_{\alpha \beta}\right)$ is the $S O\left(1, n_{T}\right)$ invariant constant metric with mostly minus signature. In the six-dimensional F-theory models we consider here this matrix $\Omega_{\alpha \beta}$ is identified with the intersection numbers on the base, i.e.

$$
\Omega_{\alpha \beta}=\eta_{\alpha \beta} .
$$

As described in section 2.2 one then has the non-constant, positive-definite metric

$$
g_{\alpha \beta}=2 \hat{\jmath}_{\alpha} \hat{\jmath}_{\beta}-\eta_{\alpha \beta}, \quad \hat{\jmath}_{\alpha} \equiv \eta_{\alpha \beta} \hat{\jmath}^{\beta} .
$$

The gauge-invariant field-strength $\hat{G}^{\alpha}$ is defined by

$$
\hat{G}^{\alpha}=\mathrm{d} \hat{B}^{\alpha}+\frac{1}{8} c^{\alpha} \hat{\omega}_{\text {grav }}^{\mathrm{CS}}, \quad \hat{\omega}_{\text {grav }}^{\mathrm{CS}}=\operatorname{tr}\left(\hat{\omega} \wedge \mathrm{d} \hat{\omega}+\frac{2}{3} \hat{\omega} \wedge \hat{\omega} \wedge \hat{\omega}\right),
$$

where $\hat{\omega}$ is the six-dimensional spin connection. 
Hypermultiplets. Every hypermultiplet contains four real scalars, such that we denote the scalars collectively by $\hat{q}^{U}\left(U=1, \ldots, 4 n_{H}\right)$. The hypermultiplets have a geometric interpretation as coordinates on a quaternionic manifold, whose metric is denoted by $h_{U V}$. Since we do not include vector multiplets in our setting and therefore the hypermultiplets are neutral, we will not need any further information about the hypermultiplets.

Standard form of 6D (1,0) supergravity. We choose conventions $\kappa_{6}^{2}=(2 \pi)^{3}$ such that the bosonic part of the standard $\mathcal{N}=(1,0)$ supergravity theory takes the form 37,135

$$
\begin{aligned}
S^{(6)}=\frac{1}{(2 \pi)^{3}} \int_{M_{6}}\left[\frac{1}{2} \hat{R} \hat{*} 1\right. & -\frac{1}{4} g_{\alpha \beta} \hat{G}^{\alpha} \wedge \hat{*} \hat{G}^{\beta}-\frac{1}{2} g_{\alpha \beta} \mathrm{d} \hat{\jmath}^{\alpha} \wedge \hat{*} \mathrm{~d} \hat{\jmath}^{\beta} \\
& \left.\quad-h_{U V} \mathrm{~d} \hat{q}^{U} \wedge \hat{*} \mathrm{~d} \hat{q}^{V}-\frac{1}{8} \eta_{\alpha \beta} c^{\alpha} \hat{B}^{\beta} \wedge \operatorname{tr} \hat{\mathcal{R}} \wedge \hat{\mathcal{R}}\right] .
\end{aligned}
$$

The last term in $\sqrt{3.18}$ is a Green-Schwarz term which ensures gauge invariance at one-loop level 110,141 and $\hat{\mathcal{R}}$ denotes the curvature two-form in six dimensions. This higher curvature term in F-theory can be understood via its counterpart in M-theory [37, 142, 143], as well as from higher-curvature corrections on D7-branes and O7-planes. The latter perspective will be briefly explained in section 3.3.2. The field strengths satisfy non-standard Bianchi-identities

$$
\mathrm{d} \hat{G}^{\alpha}=\frac{1}{8} c^{\alpha} \operatorname{tr} \hat{\mathcal{R}} \wedge \hat{\mathcal{R}}
$$

and the (anti-)-self-duality constraints for the tensors of the tensor- and gravity multiplets, which are imposed at the level of the equations of motion, are collectively given $b y 5$

$$
g_{\alpha \beta} \hat{*} \hat{G}^{\beta}=\eta_{\alpha \beta} \hat{G}^{\beta} .
$$

This six-dimensional pseudo-action will be the starting point, similar to the approach to the macroscopic description in 76 .

\subsubsection{Black string solution}

As we have seen in section 2.4.5 one can construct black string solutions of twoderivative six-dimensional $(1,0)$ supergravity coupled to tensor multiplets. There we gave an example of a black string solution with flat asymptotics. In this chapter

$\overline{5}$ Note that these are opposite to those of chapter 2 
we are interested in a slightly different solution, namely a black string which has the same asymptotics as $\mathbb{R} \times \mathrm{S}^{1} \times \mathrm{TN}_{m}$. The metric of such a solution falls in the class of solutions discussed in section 2.4.4. In particular, the metric we are interested in has vanishing harmonic functions $H_{3}, H_{4}$ and $H_{6}$ and is given by

$$
\mathrm{d} \hat{s}_{6}^{2}=2 H^{-1} \mathrm{~d} u\left(\mathrm{~d} v-\frac{1}{2} H_{5} \mathrm{~d} u\right)+H \mathrm{~d} s_{4}^{2}
$$

with a Taub-NUT metric of (positive) charge $m \square^{6}$

$$
\mathrm{d} s_{4}^{2}=H_{2}^{-1} m^{2}(\mathrm{~d} \psi+\cos (\theta) \mathrm{d} \phi)^{2}+H_{2}\left(\mathrm{~d} r^{2}+r^{2} \mathrm{~d} \theta^{2}+r^{2} \sin ^{2}(\theta) \mathrm{d} \phi^{2}\right) .
$$

Furthermore, we have

$$
H=\left(\Omega_{\alpha \beta} H_{1}^{\alpha} H_{1}^{\beta}\right)^{1 / 2},
$$

and the harmonic functions on the base $\mathbb{R}^{3}$ of $\mathrm{TN}_{m}$, denoted by $H_{1}^{\alpha}, H_{2}$ and $H_{5}$, are given by

$$
H_{1}^{\alpha}=\mu_{\infty}^{\alpha}+\frac{Q^{\alpha}}{4 r}, \quad H_{2}=m_{\infty}+\frac{m}{r}, \quad H_{5}=-1+\frac{n}{r} .
$$

We also impose the restriction

$$
\Omega_{\alpha \beta} \mu_{\infty}^{\alpha} \mu_{\infty}^{\beta}=1
$$

in order to get the right asymptotics. The coordinate ranges are given by $0 \leq u<\ell$ for a length $\ell,-\infty<v<\infty, 0 \leq r<\infty, 0 \leq \psi<\frac{4 \pi}{m}, 0 \leq \theta<\pi$ and $0 \leq \phi<2 \pi$. We will use the following dreibein for the $\psi, \phi, \theta$ part of the metric (which we henceforth will refer to as the spherical part $\mathrm{TN}_{m}^{\mathrm{sph}}$ )

$$
\begin{aligned}
& \hat{e}^{1}=\sqrt{H H_{2}} r(\sin (\psi) \mathrm{d} \theta-\cos (\psi) \sin (\theta) \mathrm{d} \phi), \\
& \hat{e}^{2}=\sqrt{H H_{2}} r(\cos (\psi) \mathrm{d} \theta+\sin (\psi) \sin (\theta) \mathrm{d} \phi), \\
& \hat{e}^{3}=\sqrt{H / H_{2}} m(\mathrm{~d} \psi+\cos (\theta) \mathrm{d} \phi) .
\end{aligned}
$$

The near-horizon geometry of the metric 3.21) which is obtained in the limit $r \rightarrow 0$ is $\mathrm{AdS}_{3} \times \mathrm{S}^{3} / \mathbb{Z}_{m}$ with the radius of $\mathrm{S}^{3} / \mathbb{Z}_{m}$ given by $R^{2}=m \sqrt{\Omega_{\alpha \beta} Q^{\alpha} Q^{\beta}}$. In addition to a non-trivial metric background the solution also requires a radial profile for the scalars $\hat{\jmath}^{\alpha}$ given by

$$
\hat{\jmath}^{\alpha}=\frac{H_{1}^{\alpha}}{H},
$$

\footnotetext{
${ }^{6}$ Here we choose coordinates for the Taub-NUT space which differ from the ones usually found in the literature.
} 
and non-vanishing three-form backgrounds 7

$$
\hat{G}^{\alpha}=-\mathrm{d} v \wedge \mathrm{d} u \wedge \mathrm{d}\left(H_{1}^{\alpha} H^{-2}\right)-*_{4} \mathrm{~d}\left(H_{1}^{\alpha}\right),
$$

where $*_{4}$ denotes the Hodge dual with respect to the Taub-NUT metric $\mathrm{d} s_{4}^{2}$. We also note that all hypermultiplet scalars are taken to be constant whereas all fermions vanish in the background.

Let us comment on the geometric properties of the Taub-NUT space 3.22. Firstly, we note that the Taub-NUT space has conical singularities for $m>1$. In order to avoid these one can consider multi-centered solutions. One can see the metric 3.22 for general $m$ as an $m$-centered Taub-NUT space in the limit in which all centers are taken to be coincident. The singularity then arises from the collapsing two-cycles between the centers of the multi-centered Taub-NUT space. Secondly, we recall that topologically Taub-NUT space is a circle fibration over $\mathbb{R}^{3}$ and the radius of the circle at infinity is $r_{\infty}=1 / \sqrt{m_{\infty}}$. Varying this parameter $r_{\infty}$ there are two interesting limits which one can consider. The first limit arises when $m_{\infty} \ll \frac{m}{r}$, i.e. the NUT-circle decompactifies. In this limit the metric 3.22 approaches (after an additional coordinate transformation) the metric on $\mathbb{R}^{4} / \mathbb{Z}_{m}$. In particular, for the case $m=1$ one recovers the black string in flat space. The opposite limit is approached when $m_{\infty} \gg \frac{m}{r}$. This limit is implemented if the circle radius $r_{\infty}$ is much smaller than the typical length scale of $\mathbb{R}^{3}$ and leads to an effective dimensional reduction of the six-dimensional theory on this circle.

The charges corresponding to the three-forms can be calculated by integrating over the spherical part

$$
-(2 \pi)^{2} Q^{\alpha}=\int_{\mathrm{TN}_{m}^{\mathrm{sph}}} \hat{G}^{\alpha}
$$

and are related to the microscopic charges $q^{\alpha}$ via

$$
Q^{\alpha}=q^{\alpha}-\frac{m}{2} c^{\alpha}
$$

as we will demonstrate in the following. Consider type IIB supergravity compactified on the Kähler surface $B$, which is the base of the elliptically fibered $\mathrm{CY}_{3}$ in F-theory. Working in conventions $\operatorname{vol}(B)=\frac{1}{2}$ and $\ell_{s}^{2}=2 \pi$ one can expand the type IIB R-R four-form $C_{4}$ and the Kähler form $J_{B}$ of the base $B$ in harmonic $(1,1)$-forms on $B$

$$
C_{4}=\hat{B}^{\alpha} \wedge \omega_{\alpha}, \quad J_{B}=\hat{\jmath}^{\alpha} \omega_{\alpha}, \quad \text { with } \quad \omega_{\alpha} \in H^{1,1}(B) .
$$

\footnotetext{
${ }^{7}$ Note that the last term comes with a minus sign instead of the plus sign in 2.130 since the (anti-)self-duality conventions in this chapter are opposite to those of chapter 2
} 
The two-forms $\hat{B}^{\alpha}$ are upon dimensional reduction on $B$ identified with the (anti)self-dual tensors in the six-dimensional gravity- and tensor multiplets, whereas the Kähler moduli $\hat{\jmath}^{\alpha}$ are interpreted as the scalars in the tensor multiplets. In addition to the bulk type IIB supergravity action there are also localized sources, namely D3-branes, D7-branes and O7-planes, in our setup. The presence of these ten-dimensional localized sources leads to additional six-dimensional couplings, which are crucial for the identification of the macroscopic with the microscopic charges. The D3-brane action contains the standard Chern-Simons action. Now consider $N$ D3-branes with worldvolume $W_{\mathrm{D} 3}=\Sigma \times C$, where $\Sigma$ is a two-dimensional worldsheet in the six uncompactified dimensions and $C \subset B$ is the curve in the base. Dimensionally reducing the Chern-Simons coupling we obtain

$$
\begin{aligned}
S_{\text {string }}^{\mathrm{CS}} & =-\frac{N}{2 \pi} \int_{W_{\mathrm{D} 3}} C_{4}=-\frac{N}{2 \pi} \int_{\Sigma} \hat{B}^{\alpha} \int_{C} \omega_{\alpha} \\
& =-\frac{N}{2 \pi} \int_{\Sigma} \hat{B}^{\alpha} q^{\beta} \int_{B} \omega_{\alpha} \wedge \omega_{\beta}=-\frac{N}{2 \pi} \int_{\Sigma} \eta_{\alpha \beta} q^{\alpha} \hat{B}^{\beta}
\end{aligned}
$$

for the string in six dimensions arising from wrapping the D3-brane over the curve $C$. We obtain further six-dimensional couplings of the two-forms $\hat{B}^{\alpha}$ by taking into account higher curvature corrections on D7-branes and O7-planes. Expanding again the type IIB four-form $C_{4}=\hat{B}^{\alpha} \wedge \omega_{\alpha}$ and summing over all higher-curvature contributions from D7-branes and O7-planes, as dictated by the F-theory analogue of the D7-brane tadpole cancellation condition (see e.g. [144)

$$
[\mathrm{D} 7]+2[\mathrm{O} 7]=12 c_{1}(B)
$$

one obtains the six-dimensional higher-curvature term relevant in the generalized Green-Schwarz mechanism 3.18). The total six-dimensional action is then the bulk part 3.18 coupled to the localized action 3.32

$$
S_{\mathrm{tot}}^{(6)}=S^{(6)}+S_{\mathrm{string}}^{\mathrm{CS}} \text {. }
$$

Deriving the equations of motion of the (anti-)self-dual tensors we obtain ${ }^{8}$

$$
\mathrm{d}\left(g_{\alpha \beta} \hat{*} \hat{G}^{\beta}\right)=(2 \pi)^{2} N \eta_{\alpha \beta} q^{\beta} \delta(\Sigma)+\frac{1}{8} \eta_{\alpha \beta} c^{\beta} \operatorname{tr} \hat{\mathcal{R}} \wedge \hat{\mathcal{R}}
$$

where $\delta(\Sigma)$ is a four-form delta current localized on the worldsheet of the six-

${ }^{8}$ We followed footnote 6 in 145 and implement the self-duality of the tensors by effectively
dividing the source terms in the naive equations of motion derived from 3.34 by a factor two. 
dimensional string. Integrating the resulting equation over $\mathrm{TN}_{m}$ leads to 9

$$
\begin{aligned}
\frac{1}{(2 \pi)^{2}} \int_{\mathrm{TN}_{m}} \mathrm{~d}\left(g_{\alpha \beta} \hat{*} \hat{G}^{\beta}\right) & =\frac{1}{(2 \pi)^{2}} \eta_{\alpha \beta} \int_{\mathrm{TN}_{m}} \mathrm{~d} \hat{G}^{\beta}=-\frac{1}{(2 \pi)^{2}} \int_{\mathrm{TN}_{m}^{\mathrm{sph}}} \eta_{\alpha \beta} \hat{G}^{\beta}=\eta_{\alpha \beta} Q^{\beta} \\
& =N \eta_{\alpha \beta} q^{\beta}+\frac{1}{8} \frac{1}{(2 \pi)^{2}} \eta_{\alpha \beta} c^{\beta} \int_{\mathrm{TN}_{m}} \operatorname{tr} \hat{\mathcal{R}} \wedge \hat{\mathcal{R}}
\end{aligned}
$$

Using furthermore that the first Pontryagin number of Taub-NUT is given by

$$
p_{1}\left(\mathrm{TN}_{m}\right)=-\frac{1}{2} \frac{1}{(2 \pi)^{2}} \int_{\mathrm{TN}_{m}} \operatorname{tr} \hat{\mathcal{R}} \wedge \hat{\mathcal{R}}=2 m
$$

we arrive at

$$
Q^{\alpha}=N q^{\alpha}-\frac{m}{2} c^{\alpha},
$$

which is the desired relation between the macroscopic charge $Q^{\alpha}$ and the microscopic charge $q^{\alpha}$. Most importantly, the classical two-derivative relation $Q^{\alpha}=q^{\alpha}$ obtains a shift proportional to the first Chern class of the base due to the non-trivial topology of the transverse Taub-NUT space.

The relation between the macroscopic and microscopic charges 3.30 can also be derived from making contact with the five-dimensional M-theory description. This can be achieved by doing the reduction along the NUT-circle parametrized by $\psi$ to five dimensions 37. The ansatz for the three-forms is given by

$$
\hat{G}^{\alpha}=G^{\alpha}-F^{\alpha} \wedge\left[m(\mathrm{~d} \psi+\cos (\theta) \mathrm{d} \phi)+A^{0}\right],
$$

where $A^{0}$ is the Kaluza-Klein gauge field and $G^{\alpha}$ is a five-dimensional three-form. The five-dimensional field strengths are defined in terms of their two-form potentials and the vectors by

$$
G^{\alpha}=\mathrm{d} B^{\alpha}+A^{\alpha} \wedge F^{0}
$$

where $F^{0}=\mathrm{d} A^{0}$. The three-forms $G^{\alpha}$ are related to the two-forms $F^{\alpha}=\mathrm{d} A^{\alpha}$ via the duality relation 3.20 . In order to match the M-theory reduction one has to identify the field strengths $F^{\alpha}$ in terms of the original M-theory field strengths $F_{M}^{\alpha}=\mathrm{d} A_{M}^{\alpha}, F_{M}^{0}=\mathrm{d} A_{M}^{0}$ via the relation 37.

$$
F^{\alpha}=\frac{1}{2}\left(F_{M}^{\alpha}-\frac{1}{2} c^{\alpha} F_{M}^{0}\right) .
$$

To be more precise, $F_{M}^{\alpha}, F_{M}^{0}$ arise from expanding the M-theory four-form field strength as $G_{4}=F_{M}^{\alpha} \wedge \omega_{\alpha}+F_{M}^{0} \wedge \omega_{0}$ along vertical divisors. Using 3.41 we find

$$
Q^{\alpha}=\frac{1}{\pi} \int_{\mathrm{S}^{2}} F^{\alpha}=\frac{1}{2 \pi} \int_{\mathrm{S}^{2}}\left(F_{M}^{\alpha}-\frac{1}{2} c^{\alpha} F_{M}^{0}\right)=q^{\alpha}-\frac{m}{2} c^{\alpha} .
$$

\footnotetext{
${ }^{9}$ The minus sign after the second equality sign is because in our conventions the orientation of $\mathrm{TN}_{m}^{\mathrm{sph}}$ is not the orientation induced by $\mathrm{TN}_{m}$.
} 
The relation 3.42 between the six-dimensional macroscopic charges $Q^{\alpha}$ and the microscopic charges $q^{\alpha}$ is crucial in order to compare our supergravity results with the microscopic data $(3.3)$ and $(3.10)$. Similar shifts have been noticed in related settings 146148 .

\subsubsection{Classical contributions to central charges and levels}

In the following we will compute the classical contributions to the central charges and levels. By 'classical' we mean those contributions which can be obtained from the six-dimensional $\mathcal{N}=(1,0)$ supergravity theory describing our F-theory setup. This is done by extracting coefficients of Chern-Simons terms in three dimensions arising upon dimensionally reducing the six-dimensional action.

\subsubsection{Reduction at asymptotic infinity}

The Bekenstein-Hawking entropy of a black hole scales with the area of its event horizon and since this entropy can be calculated from the central charges and levels, one would expect that one has to do the reduction to three dimensions in the near-horizon geometry. However, black holes can have hair, in other words degrees of freedom living outside of the horizon and contributing to the microscopic degeneracy 65 66]. A well studied example is provided by considering the BMPV black hole [43, which is microscopically described by a D1-D5 system of type IIB on $\mathrm{K} 3 \times \mathrm{S}^{1}$ carrying momentum along $\mathrm{S}^{1}$ and having equal angular momentum in two planes transverse to the D5-brane. Macroscopically this is a five-dimensional rotating black hole. This BMPV black hole can be placed at the center of Taub-NUT to get a four-dimensional black hole, since Taub-NUT space with $m=1$ looks like $\mathbb{R}^{4}$ in the limit $m_{\infty} r \ll 1$. While the five-dimensional near-horizon geometries of the BMPV black hole and its Taub-NUT generalization are the same, the microscopic degeneracies were shown to be different [149]. The difference can be explained by invoking the aforementioned hair. For example the center of mass degrees of freedom of the brane system are not captured by the near-horizon geometry. Since our setting also includes a Taub-NUT space we expect non-vanishing contributions from hair which has to be taken into account to match the microscopic results of four-dimensional black holes. However, instead of explicitly constructing the hair modes as done in 65 for the BMPV black hole, we use the approach suggested in 60 and discussed in section 1.4.1. More precisely, we perform the reduction to three dimensions at asymptotic infinity, which for our setting corresponds to 
sending the dimensionless quantity

$$
r^{\prime} \equiv \frac{m_{\infty} r}{m} \rightarrow \infty
$$

Concretely, this means that the reduction is done on the spherical part at large $r^{\prime}$. For the terms involving the charges we find that in the classical supergravity reduction the only term leading to different contributions of near-horizon and asymptotic geometry is the higher derivative part of the six-dimensional action. Due to our non-trivial transverse geometry this is different from previous work 60 . where also the higher derivative part is the same in the near-horizon and asymptotic reductions. In 60 the difference of the asymptotic and near-horizon reduction manifested itself at the level of $\mathcal{O}(1)$ contributions, which we do not consider in the following.

The fact that the six-dimensional near-horizon geometry does not reproduce the microscopic results for four-dimensional black holes can also be understood from a different perspective. The microscopic derivations in M-theory have been performed in the regime where all volumes of the $\mathrm{CY}_{3}$ are sufficiently large. This in particular includes the elliptic fiber. The duality to F-theory then implies that we have to consider backgrounds on a small NUT-circle. Therefore, we expect that the solutions 3.21 can only be used to reproduce the microscopic quantities in the limit (3.43). Furthermore, the reduced six-dimensional effective action can only be matched to the five-dimensional effective action after adding one-loop corrections coming from the compactification circle. Therefore there is no classical lift of the five-dimensional black string and four-dimensional black hole solution of M-theory to the six-dimensional F-theory solution. The microscopic central charges and levels thus will not just follow from a reduction of six-dimensional supergravity on this background. However, they do follow when one also takes one-loop corrections into account coming from integrating out massive Kaluza-Klein modes on the compact space in the geometry. Calculating these one-loop effects will be the subject of section 3.4

\subsubsection{Ansatz for the reduction}

We now present our ansatz for the metric and three-form field strength in order to perform the reduction in the asymptotic geometry, given as a suitable generalization of the ansatz for the near-horizon geometry $\mathrm{AdS}_{3} \times \mathrm{S}^{3} / \mathbb{Z}_{m}$. We will do the reduction at an arbitrary radius and compare the asymptotic and near-horizon results. 
Near-horizon geometry. The near-horizon geometry of the black string solution (3.21) is $\mathrm{AdS}_{3} \times \mathrm{S}^{3} / \mathbb{Z}_{m}$. First consider the simplest case where $m=1$. This nearhorizon geometry has an $S O(4)$ isometry group which is identified with rotations on $\mathrm{S}^{3}$. Once perturbations of this background are included, the isometries are gauged and one obtains $S O(4)$ gauge fields. At the level of the algebra, one has $\mathfrak{s o}(4)=\mathfrak{s u}(2)_{L} \oplus \mathfrak{s u}(2)_{R}$, such that we effectively have two sets of $\mathfrak{s u}(2)$ gauge fields. The ansatz for the dimensional reduction on $\mathrm{AdS}_{3} \times \mathrm{S}^{3}$ can be found in e.g. 60 136. We will make use of this ansatz in the following and adapt it appropriately to our setting.

For general NUT-charge $m$ the isometry group $S O(4)$ is broken to $U(1)_{L} \times S U(2)_{R}$. The unbroken $\mathfrak{u}(1)_{L} \subset \mathfrak{s u}(2)_{L}$ algebra is generated by the generator $J_{L}^{3}$ of the original $\mathfrak{s u}(2)_{L}$ and the total algebra is generated by Killing vectors

$$
\begin{aligned}
& K_{L}=\partial_{\psi}, \\
& K_{R}^{1}=\sin (\phi) \partial_{\theta}+\cos (\phi) \cot (\theta) \partial_{\phi}-\frac{\cos (\phi)}{\sin (\theta)} \partial_{\psi}, \\
& K_{R}^{2}=\cos (\phi) \partial_{\theta}-\sin (\phi) \cot (\theta) \partial_{\phi}+\frac{\sin (\phi)}{\sin (\theta)} \partial_{\psi}, \\
& K_{R}^{3}=-\partial_{\phi},
\end{aligned}
$$

which we collectively denote by $K^{i}=\left(K_{R}^{I}, K_{L}\right)$ and similarly $A^{i}=\left(A_{R}^{I}, A_{L}\right)$, $F^{i}=\left(F_{R}^{I}, F_{L}\right)$. Let us take $\sqrt{\eta_{\alpha \beta} Q^{\alpha} Q^{\beta}}=\frac{1}{m}$ such that the Lens space has unit radius. The appropriate ansatz is $60,136{ }^{10}$

$$
\begin{aligned}
\mathrm{d} \hat{s}_{6}^{2} & =\mathrm{d} s_{\mathrm{AdS}_{3}}^{2}+\delta_{a b} e^{a} e^{b}, \\
\hat{G}^{\alpha} & =-Q^{\alpha}\left[(2 \pi)^{2} m\left(e_{3}^{(m)}-\chi_{3}\right)+\omega\left(\mathrm{AdS}_{3}\right)\right],
\end{aligned}
$$

where

$$
e_{3}^{(m)}=\frac{1}{2 \pi^{2}}\left[e^{1} \wedge e^{2} \wedge e^{3}-\frac{1}{2} K_{L a} e^{a} \wedge F_{L}+\frac{1}{2} K_{R a}^{I} e^{a} \wedge F_{R}^{I}\right]
$$

and $\omega\left(\mathrm{AdS}_{3}\right)$ a three-form on $\mathrm{AdS}_{3}$ whose form we do not specify. The dreibein is now given by

$$
e^{a}=\hat{e}^{a}-K_{L}^{a} A_{L}-K_{R}^{I a} A_{R}^{I},
$$

with $\hat{e}^{a}$ the dreibein 3.26 in the near-horizon limit $r \rightarrow 0$. The three-form $e_{3}^{(m)}$ has the same form as $e_{3}$, which is used for a reduction on the three-sphere [136],

\footnotetext{
${ }^{10}$ Strictly the expression $\sqrt{3.46}$ only satisfies the self-duality constraint after setting the gauge fields to zero. However this is sufficient as the gauge fields are treated as fluctuations around the background.
} 
but since $0 \leq \psi<\frac{4 \pi}{m}$, the integral of $e_{3}^{(m)}$ over the Lens space is given by

$$
\int_{\mathrm{S}^{3} / \mathbb{Z}_{m}} e_{3}^{(m)}=\frac{1}{m} .
$$

It is also invariant under $U(1)_{L} \times S U(2)_{R}$ transformations and one has the relation

$$
\mathrm{d} e_{3}^{(m)}=\frac{1}{16 \pi^{2}} F_{L} \wedge F_{L}+\frac{1}{8 \pi^{2}} \operatorname{tr} F_{R} \wedge F_{R}
$$

The three-form $\chi_{3}$ in the ansatz $(3.46)$ is defined by

$$
\chi_{3}=\frac{1}{16 \pi^{2}} A_{L} \wedge F_{L}+\frac{1}{8 \pi^{2}} \operatorname{tr}\left(A_{R} \wedge \mathrm{d} A_{R}+\frac{2}{3} A_{R}^{3}\right),
$$

and ensures that the ansatz for the tensors satisfies the Bianchi identity.

Spherical part of Taub-NUT. Consider now a reduction on the spherical part of the metric 3.21 $\mathrm{TN}_{m}^{\mathrm{sph}}$ parametrized by $\psi, \phi, \theta$. The Killing vectors of Taub-NUT spacetime are still given by (3.44) and form $U(1)_{L} \times S U(2)_{R}$. This implies that the ansatz of the previous section for the three-forms is still suitable. The metric of course needs to be adapted and can be taken as 3.45 , but now with the vielbein $\hat{e}^{a}$ of the spherical part of Taub-NUT spacetime $\sqrt{3.26}$. The ansatz is thus a straightforward generalization of the one in the near-horizon geometry 3.45

$$
\begin{aligned}
\mathrm{d} \hat{s}_{6}^{2} & =\mathrm{d} s_{\mathcal{M}_{3}}^{2}+\delta_{a b} e^{a} e^{b}, \\
\hat{G}^{\alpha} & =-Q^{\alpha}\left[(2 \pi)^{2} m\left(e_{3}^{(m)}-\chi_{3}\right)+\omega\left(\mathcal{M}_{3}\right)\right],
\end{aligned}
$$

with the difference, that we do not take the near-horizon limit $r \rightarrow 0$ now ${ }^{11}$ The total metric is therefore $\mathrm{TN}_{m}^{\mathrm{sph}}$ fibered over the non-spherical part of the metric, denoted by $\mathcal{M}_{3}$. We will in the following use this ansatz to calculate the classical parts of the levels and central charges.

\subsubsection{Classical contribution from two- and higher derivative action}

The classical contributions stem from the six-dimensional supergravity action. Aside the leading two-derivative action, also a four-derivative coupling in six dimensions will be of importance to us. We will perform the reduction of the two- and four derivative action separately, and read off their contributions to the levels and central charges from coefficients of three-dimensional Chern-Simons terms.

\footnotetext{
${ }^{11}$ In particular, for the metric ansatz the dreibein $\hat{e}^{a}$ is now not taken to be in the $r \rightarrow 0$ limit (but for the three-form ansatz it is as defined in the previous paragraph).
} 
Two-derivative contribution. We calculate the contribution of the two-derivative action to the levels by determining the gauge variation of the reduced action under a $U(1)_{L} \times S U(2)_{R}$ gauge transformation. We will do this by integrating the variation of the six-dimensional Lagrangian over the spherical part $\mathrm{TN}_{m}^{\mathrm{sph}}$ to obtain the lower dimensional variation. Since $e_{3}^{(m)}$ is gauge invariant by construction, the only source for a variation under a combined $U(1)_{L} \times S U(2)_{R}$ gauge transformation, which is parametrized by $\Lambda$, is $\chi_{3}$. We therefore obtain ${ }^{12}$

$$
\begin{aligned}
\delta_{\Lambda} \mathcal{L}_{\mathrm{CS}}^{2 \text {-der }} *_{3} 1 & =-\frac{1}{16 \pi^{3}} \int_{\mathrm{TN}_{m}^{\mathrm{sph}}} g_{\alpha \beta} \delta_{\Lambda} \hat{G}^{\alpha} \wedge \hat{*} \hat{G}^{\beta}=\pi m^{2} \eta_{\alpha \beta} Q^{\alpha} Q^{\beta} \int_{\mathrm{TN}_{m}^{\mathrm{sph}}} \delta_{\Lambda} \chi_{3} \wedge e_{3}^{(m)} \\
& =\pi m \eta_{\alpha \beta} Q^{\alpha} Q^{\beta} \delta_{\Lambda} \chi_{3},
\end{aligned}
$$

where in the second equality we used the (anti-)self-duality condition 3.20 . The lower dimensional variation $(3.53)$ is nothing but the gauge variation of a threedimensional action of the form

$$
\begin{aligned}
S_{\mathrm{CS}}^{2 \text {-der }} & =\pi m \eta_{\alpha \beta} Q^{\alpha} Q^{\beta} \int_{\mathcal{M}_{3}} \chi_{3} \\
& =\frac{k_{L}^{2 \text {-der }}}{8 \pi} \int_{\mathcal{M}_{3}} A_{L} \wedge F_{L}+\frac{k_{R}^{2-\text { der }}}{4 \pi} \int_{\mathcal{M}_{3}} \operatorname{tr}\left(A_{R} \wedge F_{R}+\frac{2}{3} A_{R}^{3}\right),
\end{aligned}
$$

with levels and central charges

$$
\begin{aligned}
k_{L}^{2 \text {-der }} & =\frac{1}{2} m \eta_{\alpha \beta} Q^{\alpha} Q^{\beta}=\frac{1}{2} m \eta_{\alpha \beta}\left(q^{\alpha}-\frac{1}{2} m c^{\alpha}\right)\left(q^{\beta}-\frac{1}{2} m c^{\beta}\right), \\
k_{R}^{2 \text {-der }} & =\frac{1}{2} m \eta_{\alpha \beta}\left(q^{\alpha}-\frac{1}{2} m c^{\alpha}\right)\left(q^{\beta}-\frac{1}{2} m c^{\beta}\right), \\
c_{L}^{2 \text {-der }} & =c_{R}^{2 \text {-der }} .
\end{aligned}
$$

The central charges are equal because there is no gravitational Chern-Simons term in the $3 \mathrm{~d}$ action resulting from reducing the two-derivative part of the $6 \mathrm{~d}$ action.

Higher derivative contribution. In order to find the contribution to the levels and central charges stemming from higher derivative terms we consider the piece in the six-dimensional action

$$
S^{(6)} \supset \frac{1}{64 \pi^{3}} \int_{M_{6}} \eta_{\alpha \beta} c^{\alpha} \hat{G}^{\beta} \wedge \hat{\omega}_{\mathrm{grav}}^{\mathrm{CS}},
$$

where $\hat{\omega}_{\text {grav }}^{\mathrm{CS}}$ is the gravitational Chern-Simons three-form built of the $6 \mathrm{~d}$ spin connection. We will compute Chern-Simons terms in three dimensions by integrating ${ }^{12}$ In our conventions $\int_{M_{6}}=\int_{\mathcal{M}_{3}} \cdot \int_{\mathrm{TN}_{m}^{\mathrm{sph}}}$. 
(3.56) over the spherical part $\mathrm{TN}_{m}^{\mathrm{sph}}$ for general $r$, in particular not taking the nearhorizon limit. One finds

$$
\begin{aligned}
& \mathcal{L}_{\mathrm{CS}}^{4-\text { der }} *_{3} 1=\frac{1}{64 \pi^{3}} \int_{\mathrm{TN}_{m}^{\mathrm{sph}}} \eta_{\alpha \beta} c^{\alpha} \hat{G}^{\beta} \wedge \hat{\omega}_{\mathrm{grav}}^{\mathrm{CS}} \\
&=\frac{1}{16 \pi} \eta_{\alpha \beta} c^{\alpha}\left(q^{\beta}-\frac{1}{2} m c^{\beta}\right)\left[\omega_{\mathrm{grav}}^{\mathrm{CS}}-\frac{1+4{r^{\prime}}^{\prime}+2{r^{\prime}}^{2}}{\left(1+r^{\prime}\right)^{4}} A_{L} \wedge F_{L}\right. \\
&\left.+2 \frac{1+4 r^{\prime}+10{r^{\prime}}^{2}+8{r^{\prime}}^{3}+2{r^{\prime}}^{4}}{\left(1+r^{\prime}\right)^{4}} \omega^{\mathrm{CS}}\left(A_{R}\right)\right],
\end{aligned}
$$

where we used $r^{\prime}=\frac{m_{\infty}}{m} r$. For the second equality sign in 3.57 we only took the parts of $\hat{G}^{\beta}$ and $\hat{\omega}_{\text {grav }}^{\mathrm{CS}}$ that lead to Chern-Simons terms in three-dimensions. The choice of dreibein 3.26 turns out to be very important in order to get proper Chern-Simons terms after reduction. This probably has to do with whether or not the dreibein is globally defined. Details of this calculation can be found in appendix 3.A

Now there are two limits of (3.57) interesting to us: the near-horizon limit $r^{\prime} \rightarrow 0$, where we effectively go to $\operatorname{AdS}_{3} \times \mathrm{S}^{3} / \mathbb{Z}_{m}$ and the $r^{\prime} \rightarrow \infty$ limit corresponding to performing the 'reduction at infinity'. In the near-horizon limit $r^{\prime} \rightarrow 0$ we obtain

$$
\mathcal{L}_{\mathrm{CS}}^{4 \text {-der }} *_{3} 1=\frac{1}{16 \pi} \eta_{\alpha \beta} c^{\alpha}\left(q^{\beta}-\frac{1}{2} m c^{\beta}\right)\left[\omega_{\mathrm{grav}}^{\mathrm{CS}}-A_{L} \wedge F_{L}+2 \omega^{\mathrm{CS}}\left(A_{R}\right)\right]
$$

from which we read off the following contributions to the central charges and levels

$$
\begin{aligned}
k_{L}^{4-\text { der }} & =-\frac{1}{2} \eta_{\alpha \beta} c^{\alpha}\left(q^{\beta}-\frac{1}{2} m c^{\beta}\right), \\
k_{R}^{4-\text { der }} & =\frac{1}{2} \eta_{\alpha \beta} c^{\alpha}\left(q^{\beta}-\frac{1}{2} m c^{\beta}\right), \\
\left(c_{L}-c_{R}\right)^{4-\text { der }} & =6 \eta_{\alpha \beta} c^{\alpha}\left(q^{\beta}-\frac{1}{2} m c^{\beta}\right) .
\end{aligned}
$$

We used the fact that the coefficient of the three-dimensional gravitational ChernSimons term determines the difference between the left- and right-moving central charges. The latter difference can be read off from the gravitational Chern-Simons term by comparing it to

$$
\mathcal{L}_{\mathrm{CS}} *_{3} 1 \supset \frac{c_{L}-c_{R}}{96 \pi} \omega_{\text {grav }}^{\mathrm{CS}}
$$

Setting $m=1$ and dropping the charge shift, this is the result obtained in 60,76 . The shift in the charges is absent in these settings, which involve black holes in asymptotically flat spacetime, as opposed to our case. We therefore recover prefactors which are in agreement with their results. 
The near-horizon results 3.59 turn out not to give the correct classical higher derivative correction to the central charges and levels. In contrast, taking the limit $r^{\prime} \rightarrow \infty$ in 3.57 one finds

$$
\mathcal{L}_{\mathrm{CS}}^{4-\mathrm{der}} *_{3} 1=\frac{1}{16 \pi} \eta_{\alpha \beta} c^{\alpha}\left(q^{\beta}-\frac{1}{2} m c^{\beta}\right)\left[\omega_{\mathrm{grav}}^{\mathrm{CS}}+4 \omega^{\mathrm{CS}}\left(A_{R}\right)\right],
$$

such that we obtain

$$
\begin{aligned}
k_{L}^{4-\text { der }} & =0, \\
k_{R}^{4-\text { der }} & =\eta_{\alpha \beta} c^{\alpha}\left(q^{\beta}-\frac{1}{2} m c^{\beta}\right), \\
\left(c_{L}-c_{R}\right)^{4-\text { der }} & =6 \eta_{\alpha \beta} c^{\alpha}\left(q^{\beta}-\frac{1}{2} m c^{\beta}\right) .
\end{aligned}
$$

The total classical contributions from the reduction in the asymptotic geometry are therefore given by

$$
\begin{aligned}
k_{L}^{\text {class }} & =\frac{1}{2} m C^{2}-\frac{1}{2} m^{2} c_{1}(B) \cdot C+\frac{1}{8} m^{3} c_{1}(B)^{2}, \\
k_{R}^{\text {class }} & =\frac{1}{2} m C^{2}-\frac{1}{2} m^{2} c_{1}(B) \cdot C+\frac{1}{8} m^{3} c_{1}(B)^{2}+c_{1}(B) \cdot C-\frac{1}{2} m c_{1}(B)^{2}, \\
\left(c_{L}-c_{R}\right)^{\text {class }} & =6 c_{1}(B) \cdot C-3 m c_{1}(B)^{2} .
\end{aligned}
$$

This is obviously not the full answer, as it does not match the microscopic results (3.3) and (3.10). The mismatch is not surprising because we know that in order to match the six- and five-dimensional effective actions one has to add one-loop corrections to the dimensionally reduced six-dimensional action 37,119$]$. The results (3.63) are actually equal to the central charges and levels one would find from the five-dimensional action before adding these one-loop corrections. To reproduce the microscopic results one also has to include the one-loop Chern-Simons terms that arise from integrating out the massive Kaluza-Klein modes. This is what we will do in the next section. Adding the classical asymptotic contributions derived in this section to the one-loop induced contributions will lead to a matching of microscopic and macroscopic quantities up to linear order in the charges $\left(q^{\alpha}, m\right)$.

\subsection{Macroscopic quantum contributions}

We now wish to include one-loop Chern-Simons terms in three dimensions and interpret them as additional contributions to the central charges and levels. These loop-induced Chern-Simons terms arise from integrating out massive Kaluza-Klein 
(KK) modes, which run in the loops of the relevant two-point functions. Since Chern-Simons terms are intimately linked to anomalies in higher dimensions, we anticipate that the relevant three-dimensional fields to be integrated out are KK modes of chiral fields in six dimensions, which can contribute to anomalies. These fields include the six-dimensional gravitino, spin- $\frac{1}{2}$ fermions in the tensorand hypermultiplets, and the (anti-)self-dual two-forms. Upon reduction to three dimensions these fields lead to massive spin- $\frac{3}{2}$, spin- $\frac{1}{2}$, and three-dimensional chiral vector fields. These somewhat exotic chiral, (anti-)self-dual vector fields in three dimensions were first discussed in 150 . One loop corrections due to massive chiral vectors and higher rank tensors were studied in [151].

We calculate the loop-induced Chern-Simons terms in the near-horizon geometry, but argue that the result is still valid for a reduction at asymptotic infinity. To do the calculation, we first determine the relevant KK spectrum for our case by truncating the KK spectrum found in 152,153 for the case of $\mathcal{N}=(2,0)$ supergravity on $\mathrm{AdS}_{3} \times \mathrm{S}^{3}$ to the corresponding $\mathcal{N}=(1,0)$ spectrum, at least at the two-derivative level. Besides the local Lorentz group representations of the massive fields in three dimensions, we also extract the representations of the fields under the (gauged) $\mathfrak{s o}(4)=\mathfrak{s u}(2)_{L} \oplus \mathfrak{s u}(2)_{R}$ isometry of $\mathrm{S}^{3}$, as well as the signs of the three-dimensional masses. We then determine the contribution of a single field for each type to the three-dimensional $\mathfrak{u}(1)_{L}, \mathfrak{s u}(2)_{R}$ and gravitational Chern-Simons terms. Instead of computing these single field contributions in a direct loop calculation, we make use of the Atiyah-Patodi-Singer (APS) index theorem 154 156. Armed with these results we then sum the contributions over all KK towers and determine the total contribution employing zeta-function regularization. In particular, we implement the $\mathbb{Z}_{m}$ quotient in the sum over KK states. Adding these quantum corrections to the classical ones obtained in section 3.3 we find agreement with the microscopic result up to and including terms of linear order in the charges.

\subsubsection{Kaluza-Klein spectrum}

We now determine the $\mathfrak{s u}(2)_{L} \oplus \mathfrak{s u}(2)_{R}$ representations of the massive spin- $\frac{1}{2}$, spin- $\frac{3}{2}$ and two-form Kaluza-Klein modes before taking the $\mathbb{Z}_{m}$ quotient. The six-dimensional fields that give rise to relevant Kaluza-Klein modes are the gravitino and self-dual two-form in the gravity multiplet, the tensorinos and anti-self-dual tensors in the tensor multiplets, and the hyperinos in the hypermultiplets. The gravitino, tensorinos and hyperinos are all given by two Weyl fermions subject to a symplectic-Majorana condition. The tensors obey a reality condition. The 
$\mathcal{N}=(1,0)$ theory coupled to tensor multiplets can be obtained as a truncation of the $\mathcal{N}=(2,0)$ theory. The spectrum of $\mathcal{N}=(2,0)$ supergravity on $\mathrm{S}^{3}$ was worked out in 152 153. The extra content we have are the hypermultiplets, but for now we assume that the modes associated to the fermions in these multiplets fall in the same representations as the fermions in the tensor multiplets. We now list the massive modes that one gets without taking into account the symplectic-Majorana and reality conditions and denote the spectrum in terms of $\mathfrak{s o}(4)=\mathfrak{s u}(2)_{L} \oplus \mathfrak{s u}(2)_{R}$ representations $\left(j_{L}, j_{R}\right)^{\operatorname{sgn}(M)} 152,153[13$ where $\operatorname{sgn}(M)$ denotes the sign of the mass.

- Spin- $\frac{3}{2}$ :

$$
2 \bigoplus_{j_{L}=\frac{1}{2}}^{\infty}\left(j_{L}, j_{L} \pm \frac{1}{2}\right)^{\mp}
$$

- Spin- $\frac{1}{2}$ :

$$
\begin{gathered}
2 \bigoplus_{j_{L}=\frac{3}{2}}^{\infty}\left(j_{L}, j_{L} \pm \frac{3}{2}\right)^{\mp} \oplus 2 \bigoplus_{j_{L}=0}^{1}\left(j_{L}, j_{L}+\frac{3}{2}\right)^{-} \oplus 2 \bigoplus_{j_{L}=1}^{\infty}\left(j_{L}, j_{L} \pm \frac{1}{2}\right)^{ \pm} \oplus 2\left(\frac{1}{2}, 1\right)^{+} \\
\oplus 2\left(0, \frac{1}{2}\right)^{+} \oplus 2\left(n_{T}+n_{H}\right) \bigoplus_{j_{L}=\frac{1}{2}}^{\infty}\left(j_{L}, j_{L} \pm \frac{1}{2}\right)^{ \pm} \oplus 2\left(n_{T}+n_{H}\right)\left(0, \frac{1}{2}\right)^{+} .
\end{gathered}
$$

- Chiral vectors:

$$
\begin{aligned}
\bigoplus_{j_{L}=1}^{\infty}\left(j_{L}, j_{L} \pm 1\right)^{\mp} & \oplus\left(\frac{1}{2}, \frac{3}{2}\right)^{-} \oplus(0,1)^{-} \oplus n_{T} \bigoplus_{j_{L}=1}^{\infty}\left(j_{L}, j_{L} \pm 1\right)^{ \pm} \\
& \oplus n_{T}\left(\frac{1}{2}, \frac{3}{2}\right)^{+} \oplus n_{T}(0,1)^{+}
\end{aligned}
$$

The notation we use to denote the representations of the massive KK states is analogous to the notation used in 153 . In particular the notation

$$
\left(j_{L}, j_{L} \pm \frac{1}{2}\right)^{\mp}=\left(j_{L}, j_{L}+\frac{1}{2}\right)^{-} \oplus\left(j_{L}, j_{L}-\frac{1}{2}\right)^{+}
$$

is a shorthand notation for the existence of two infinite towers of KK modes in the spectra listed above. We furthermore want to mention that the three-dimensional fermions are Dirac spinors and the chiral vectors are complex.

\footnotetext{
${ }^{13}$ In 152 the spectrum is denoted in terms of the highest weight vector $\left(l_{1}, l_{2}\right)$ of $\mathfrak{s o}(4)$ which is related to our notation by $l_{1}=j_{L}+j_{R}, l_{2}=j_{L}-j_{R}$.
} 
Applying the symplectic-Majorana and reality conditions for the gravitino and the tensors means that modes with quantum numbers $j_{L}^{3}, j_{R}^{3}$ are mapped to modes with quantum numbers $-j_{L}^{3},-j_{R}^{3}$ 157. Here $j_{L}^{3}, j_{R}^{3}$ are the eigenvalues of the generators of $\mathfrak{u}(1)_{L} \subset \mathfrak{s u}(2)_{L}$ and $\mathfrak{u}(1)_{R} \subset \mathfrak{s u}(2)_{R}$ respectively. This effectively means that we only have to sum over modes with $j_{L}^{3} \geq 0$. The Kaluza-Klein spectrum for a reduction on $\mathrm{S}^{3} / \mathbb{Z}_{m}$ can then be obtained by projecting onto the $\mathbb{Z}_{m}$-invariant states of the spectrum on $\mathrm{S}^{3}$, as shown above. As we show in appendix 3.B this means that we only keep those states which have $j_{L}^{3}=\frac{1}{2} m k$ for some $k \in \mathbb{Z}_{\geq 0}$, see also 157 .

If one performs the reduction in the asymptotic geometry one reduces on a squashed Lens space, where the radius of the two-sphere inside the squashed threedimensional geometry is taken to be large. We expect that the representation content of the KK spectrum does not get altered by the squashing. Note that, due to the asymptotic NUT-circle, the masses of the Kaluza-Klein modes remain finite. In addition we assume, that the squashing of the $S^{3} / \mathbb{Z}_{m}$ does not change the sign of the mass of the KK states. These assumptions essentially imply, that we can do the loop computation in the near-horizon geometry and use the spectrum on $\mathrm{S}^{3} / \mathbb{Z}_{m}$.

\subsubsection{One-loop Chern-Simons terms from KK spectrum}

Quantum corrections to Chern-Simons terms can be interpreted as compensations for the parity violation introduced by families of massive fields, after they are integrated out [119]. The fields that contribute in our case to the three-dimensional parity anomaly are massive spin- $\frac{1}{2}$ fermions, spin- $\frac{3}{2}$ fermions and massive vectors in three dimensions. We can thus calculate these corrections by calculation of the parity-violating piece of the effective action which can be expressed using the Atiyah-Patodi-Singer $\eta$-invariant 158 corresponding to the relevant Dirac operator. This $\eta$-invariant can be expressed in Chern-Simons terms by extending the Dirac operator to one dimension higher and using the Atiyah-Patodi-Singer index theorem [158. This calculation is valid for three-dimensional Riemannian manifolds of the form $\mathcal{M}_{3}=\mathbb{R} \times \mathcal{M}_{2}$, where $\mathcal{M}_{2}$ is a compact manifold without boundary. Since we are doing the reduction at infinity, where the three-dimensional manifold (after Wick rotation) is of the form $\mathbb{R}^{2} \times \mathrm{S}^{1}$, the index theorem is indeed applicable by treating this manifold as $\mathbb{R} \times \mathrm{S}_{R}^{1} \times \mathrm{S}^{1}$, where we take the radius of the $\mathrm{S}_{R}^{1}$ circle to be very large.

We now first treat the spin- $\frac{1}{2}$ fermions, the spin- $\frac{3}{2}$ fermions and the massive 
vectors separately. The loop corrections induced by these three types of fields are listed in table 3.1. After these corrections are determined, we sum the latter over the spectrum determined in the preceding subsection to compute the full one-loop correction to the central charges and levels.

Spin- $\frac{\mathbf{1}}{\mathbf{2}}$ fermions. We consider a massive spin- $\frac{1}{2}$ fermion coupled to the gauge fields $A=\left(A_{L}, A_{R}\right)$ taking values in the Lie algebra $\mathfrak{u}(1)_{L} \oplus \mathfrak{s u}(2)_{R}$ and to an external gravitational field denoted by the vielbein $e$ with spin connection $\omega$. The parity anomaly resulting from this particle can be canceled by a term 158 .

$$
-i \pi \operatorname{sgn}(M) \int_{\mathcal{M}_{3}} Q_{\frac{1}{2}}(A, \omega)
$$

with

$$
\mathrm{d} Q_{\frac{1}{2}}(A, \omega)=\hat{A}\left(\mathcal{M}_{3}\right) \wedge \operatorname{ch}\left(F_{L}\right) \wedge \operatorname{ch}\left(F_{R}\right) \mid,
$$

where the vertical dash denotes that we pick out the four-form contribution of the whole expansion on the right hand side of 4.42). The form at the right-hand side is the index density appropriate to the Dirac operator for spin- $\frac{1}{2}$ particles in four dimensions. It is expressed in terms of the Dirac genus $\hat{A}$ and Chern character, which have an expansion

$$
\begin{aligned}
\hat{A}\left(\mathcal{M}_{3}\right) & =1+\frac{1}{(4 \pi)^{2}} \frac{1}{12} \operatorname{tr} \mathcal{R} \wedge \mathcal{R}+\ldots, \\
\operatorname{ch}(F) & =r+\frac{i}{2 \pi} \operatorname{tr} F-\frac{1}{2} \frac{1}{(2 \pi)^{2}} \operatorname{tr} F \wedge F-\frac{i}{6} \frac{1}{(2 \pi)^{3}} \operatorname{tr} F \wedge F \wedge F+\ldots,
\end{aligned}
$$

where $r$ is the dimension of the representation of the gauge group, under which the spin- $\frac{1}{2}$ fermion transforms. We use that

$$
\hat{A}\left(\mathcal{M}_{3}\right) \wedge \operatorname{ch}\left(F_{L}\right) \wedge \operatorname{ch}\left(F_{R}\right) \mid=\frac{1}{(2 \pi)^{2}}\left(\frac{r}{4} F_{L} \wedge F_{L}-\frac{1}{2} \operatorname{tr} F_{R} \wedge F_{R}+\frac{r}{48} \operatorname{tr} \mathcal{R} \wedge \mathcal{R}\right)
$$

where now $r$ is the dimension of the $\mathfrak{s u}(2)_{R}$ representation of the spin- $\frac{1}{2}$ fermion and we used that the generator for the $\mathfrak{u}(1)_{L}$ is given in terms of the Pauli matrices by $-\frac{i}{2} \sigma_{3}$. We find that the counterterm to cancel the parity anomaly is then given by

$$
\operatorname{sign}(M)\left(-\frac{i r}{16 \pi} A_{L} \wedge F_{L}+\frac{i}{8 \pi} \omega_{\mathrm{CS}}\left(A_{R}\right)-\frac{i r}{192 \pi} \omega_{\text {grav }}^{\mathrm{CS}}\right) .
$$

Note that these are the corrections to the action on the Riemannian manifold. We still have to Wick rotate to Lorentzian signature by multiplying with a factor $i$, 
which yields the counter terms

$$
\operatorname{sign}(M)\left(\frac{r}{16 \pi} A_{L} \wedge F_{L}-\frac{1}{8 \pi} \omega_{\mathrm{CS}}\left(A_{R}\right)+\frac{r}{192 \pi} \omega_{\text {grav }}^{\mathrm{CS}}\right) .
$$

Spin- $\frac{\mathbf{3}}{\mathbf{2}}$ fermions. For spin- $\frac{3}{2}$ fermions the counterterm is given by 3.65 with 158,159

$$
\mathrm{d} Q_{\frac{3}{2}}(A, \omega)=\hat{A}\left(\mathcal{M}_{3}\right) \wedge\left[\operatorname{tr} \exp \left(\frac{i \mathcal{R}}{2(2 \pi)^{2}}\right)-1\right] \wedge \operatorname{ch}\left(F_{L}\right) \wedge \operatorname{ch}\left(F_{R}\right) \mid .
$$

Using that $\operatorname{tr} \exp \left(\frac{i \mathcal{R}}{2 \pi}\right)-1=3-\frac{1}{2(2 \pi)^{2}} \operatorname{tr} \mathcal{R} \wedge \mathcal{R}+\ldots$, we find that

$$
\mathrm{d} Q_{\frac{3}{2}}(A, \omega)=\frac{3 r}{4(2 \pi)^{2}} F_{L} \wedge F_{L}-\frac{3}{2(2 \pi)^{2}} \operatorname{tr} F_{R} \wedge F_{R}-\frac{7 r}{16(2 \pi)^{2}} \operatorname{tr} \mathcal{R} \wedge \mathcal{R} .
$$

The counterterm to the Lorentzian action then becomes

$$
\operatorname{sign}(M)\left(\frac{3 r}{16 \pi} A_{L} \wedge F_{L}-\frac{3}{8 \pi} \omega_{\mathrm{CS}}\left(A_{R}\right)-\frac{7 r}{64 \pi} \omega_{\text {grav }}^{\mathrm{CS}}\right) .
$$

Chiral vectors. In this case we were unaware of the existence of an appropriate index theorem in the literature. When ignoring the gauge fields one gets 159

$$
\text { ind } i D_{A}=\frac{1}{2} \int_{M} L(M) \mid \text {, }
$$

where the Hirzebruch $L$-polynomial is given by

$$
L(M)=1-\frac{1}{(2 \pi)^{2}} \frac{1}{6} \operatorname{tr} \mathcal{R} \wedge \mathcal{R}+\ldots
$$

The equality in 3.74 only holds for the four-form and we multiplied the right hand side by two with respect to the result in 159 since we consider complex instead of real vector fields. However, we now use that the $L$-polynomial according to the Hirzebruch signature theorem also determines the Hirzebruch signature:

$$
\tau=\int_{M} L(M) \mid .
$$

If one instead considers a tensor product with another vector bundle the Hirzebruch theorem becomes 160

$$
\tau=\int_{M} L(M) \wedge \operatorname{ch}(2 F) \mid
$$


Based on these considerations, we now postulate that

$$
\text { ind } i D_{A}=\frac{1}{2} \int_{M} L(M) \wedge \operatorname{ch}(2 F) \mid \text {. }
$$

In 119 one-loop corrections are computed that one gets when integrating out massive chiral Kaluza-Klein modes after the reduction from six to five dimensions on a circle. The authors do this by explicit calculation of the diagrams. In appendix 3.C we reproduce these results using the index theorems in which we also use the index 3.78 . This is some non-trivial evidence that this is the right quantity.

Using the index 3.78 the counterterm is now given by

$$
i \pi \operatorname{sign}(M) \int_{\mathcal{M}_{3}} Q_{\mathrm{vec}}(A, \omega)
$$

where

$$
\mathrm{d} Q_{\mathrm{vec}}(A, \omega)=\frac{1}{2} L(M) \wedge \operatorname{ch}\left(2 F_{L}\right) \wedge \operatorname{ch}\left(2 F_{L}\right) \mid .
$$

Notice that 3.79 has an extra minus-sign with respect to 3.65 which is caused by the vectors being bosons 159 . We then find

$$
\mathrm{d} Q_{\text {vec }}(A, \omega)=\frac{r}{2(2 \pi)^{2}} F_{L} \wedge F_{L}-\frac{1}{(2 \pi)^{2}} \operatorname{tr} F_{R} \wedge F_{R}-\frac{1}{12} \frac{r}{(2 \pi)^{2}} \operatorname{tr} \mathcal{R} \wedge \mathcal{R} .
$$

This implies that the counterterms to the Lorentzian action are given by

$$
\operatorname{sign}(M)\left(-\frac{r}{8 \pi} A_{L} \wedge F_{L}+\frac{1}{4 \pi} \omega_{\mathrm{CS}}\left(A_{R}\right)+\frac{r}{48 \pi} \omega_{\text {grav }}^{\mathrm{CS}}\right) .
$$

Corrections to the levels and central charges. Note that all the corrections above were derived for an arbitrary representation under $\mathfrak{u}(1)_{L} \oplus \mathfrak{s u}(2)_{R}$ specified by the quantum numbers $j_{L}^{3}$ and $j_{R}$. Expressing the left Chern-Simons term in the representation we used in the classical part gives a factor $2\left(j_{L}^{3}\right)^{2}$ and expressing the right Chern-Simons terms in the fundamental representation gives a factor $\frac{2}{3} j_{R}\left(j_{R}+1\right)\left(2 j_{R}+1\right)$. We also use that the dimension of the representation under $\mathfrak{s u}(2)_{R}$ is given by $2 j_{R}+1$. The constants $\alpha_{L}, \alpha_{R}, \alpha_{\text {grav }}$ in front of the Chern-Simons terms $\omega_{\mathrm{CS}}\left(A_{L}\right), \omega_{\mathrm{CS}}\left(A_{R}\right)$ and $\omega_{\text {grav }}^{\mathrm{CS}}$ are then given in table 3.1

We now sum the contributions of table 3.1 over the spectrum determined in section 3.4.1. Since the projection condition is $j_{L}^{3}=\frac{1}{2} m k$ for $k \in \mathbb{Z}_{\geq 0}$, we first sum over all representations which contain a state with $j_{L}^{3}=\frac{1}{2} m k$. These are just the representations labeled by $j_{L}=\frac{1}{2} m k, \frac{1}{2} m k+1, \ldots$. Finally, we sum over all $k \in \mathbb{Z}_{\geq 0}$. The sums we encounter are of the form $\sum_{n} f(n)$ where $n$ runs over integers 


\begin{tabular}{c|ccc}
\hline & $\operatorname{spin}-\frac{1}{2}$ & spin- $\frac{3}{2}$ & chiral vectors \\
\hline$\alpha_{L}$ & $\frac{1}{2}\left(j_{L}^{3}\right)^{2}\left(2 j_{R}+1\right)$ & $\frac{3}{2}\left(j_{L}^{3}\right)^{2}\left(2 j_{R}+1\right)$ & $-\left(j_{L}^{3}\right)^{2}\left(2 j_{R}+1\right)$ \\
$\alpha_{R}$ & $-\frac{1}{3} j_{R}\left(j_{R}+1\right)\left(2 j_{R}+1\right)$ & $-j_{R}\left(j_{R}+1\right)\left(2 j_{R}+1\right)$ & $\frac{2}{3} j_{R}\left(j_{R}+1\right)\left(2 j_{R}+1\right)$ \\
$\alpha_{\text {grav }}$ & $\frac{1}{48}\left(2 j_{R}+1\right)$ & $-\frac{7}{16}\left(2 j_{R}+1\right)$ & $\frac{1}{12}\left(2 j_{R}+1\right)$ \\
\hline
\end{tabular}

Table 3.1: Contributions of a single field to the left-, right- and gravitational ChernSimons terms. The table should be read as $\alpha_{I}=\frac{\operatorname{sgn}(M)}{4 \pi} \times($ entry of table $)$.

or half integers. We regularize the infinite, divergent sums using zeta-function regularization. In particular, we use the regularized sums

$$
\begin{aligned}
\sum_{n=1}^{\infty} 1 & =-\frac{1}{2}, & \sum_{n=1}^{\infty} n & =-\frac{1}{12}, \\
\sum_{n=1}^{\infty} n^{2} & =0, & \sum_{n=1}^{\infty} n^{3} & =\frac{1}{120} .
\end{aligned}
$$

It is worth noting that regularizing the contributions arising from integrating out infinitely many massive modes is in general very subtle ${ }^{14}$ Firstly, applying zetafunction regularization is only possible if the higher dimensional theory is anomaly free 162 . Secondly, in a theory with gravity, one expects that there is a UV cut-off in the lower dimensional theory set by the scale at which gravity becomes strongly coupled 163, 164. It turns out that the result from this regularization scheme agrees with the zeta-function regularization.

When calculating the one-loop corrections, we also make use of the identities

$$
\begin{aligned}
& n_{H}=273-29 n_{T}, \\
& n_{T}=h^{1,1}(B)-1=9-c_{1}(B)^{2},
\end{aligned}
$$

where the first one is the anomaly cancellation condition in $6 \mathrm{D}$. The relations between the coefficients $\alpha_{I}$, which we compute by performing the infinite sums over Kaluza-Klein states, and the levels and central charges are given by

$$
k_{L}=8 \pi \alpha_{L}, \quad k_{R}=4 \pi \alpha_{R}, \quad c_{L}-c_{R}=96 \pi \alpha_{\text {grav }} .
$$

$\overline{{ }^{14} \text { See } 161}$ for a recent detailed discussion. 
The explicit calculation of the sums can be found in appendix 3.D but the results are given by

$$
\begin{aligned}
k_{L}^{\text {loop }} & =-\frac{m^{3}}{8} c_{1}(B)^{2}, \\
k_{R}^{\text {loop }} & =\frac{m^{3}}{24} c_{1}(B)^{2}+\frac{m}{3} c_{1}(B)^{2}+m, \\
\left(c_{L}-c_{R}\right)^{\text {loop }} & =6 m+2 m c_{1}(B)^{2},
\end{aligned}
$$

up to terms of $\mathcal{O}(1)$ which are independent of the charges $\left(q^{\alpha}, m\right)$.

We notice that the one loop corrections to $k_{L}$ and $c_{L}-c_{R}$ differ for the two cases $m=1$ and $m>1$ at the level of the constants. This difference would disappear when adding $4\left(\frac{1}{2}, 0\right)^{-}$representations to the spin- $\frac{1}{2}$ spectrum. Since we are however interested in contributions scaling with the charges we do not comment further on this case distinction.

\subsection{Summary and 4D/5D correspondence}

We derived the central charges $c_{L, R}$ and levels $k_{L, R}$ of an $\mathcal{N}=(0,4) \mathrm{SCFT}$ corresponding to an F-theory geometry $\mathbb{R} \times \mathrm{S}^{1} \times \mathrm{TN}_{m} \times \mathrm{CY}_{3}$ with a D3-brane wrapped around $\mathrm{S}^{1} \times C$, from six-dimensional $\mathcal{N}=(1,0)$ supergravity. Combining the classical contributions 3.63 with the one loop results 3.86 leads to the total result

$$
\begin{aligned}
k_{L} & =\frac{1}{2} m C^{2}-\frac{1}{2} m^{2} c_{1}(B) \cdot C, \\
k_{R} & =\frac{1}{2} m C^{2}-\frac{1}{2} m^{2} c_{1}(B) \cdot C+\frac{1}{6} m^{3} c_{1}(B)^{2}+c_{1}(B) \cdot C+m-\frac{1}{6} m c_{1}(B)^{2}, \\
c_{L}-c_{R} & =6 c_{1}(B) \cdot C+6 m-m c_{1}(B)^{2},
\end{aligned}
$$

for the central charges, again up to $\mathcal{O}(1)$ contributions independent of the charges. We reproduced 3.3 up to constants and we reproduced 3.10 exactly.

The 4D/5D correspondence. As already mentioned in the introduction of this chapter, the setting we studied in this chapter compactified to four dimensions is the four-dimensional side of the $4 \mathrm{D} / 5 \mathrm{D}$ correspondence 133,134 . The fivedimensional side of this correspondence is given by a five-dimensional black hole with flat asymptotics which can be uplifted to the asymptotically flat string in six dimensions. Therefore, it is interesting to compare the results 3.87 with the 
macroscopic derivation of the central charges and levels of the asymptotically flat black string. The near-horizon geometry of this string is the same as in our case with NUT-charge $m=1$. The asymptotic geometry is however different in both cases. Microscopically the asymptotically flat black string was studied in $[76$ and is described by an F-theory geometry $\mathbb{R}^{1,4} \times \mathrm{S}^{1} \times \mathrm{CY}_{3}$ with a D3-brane wrapping $\mathrm{S}^{1} \times C$ for a curve $C \subset B$ in the base of the Calabi-Yau threefold. It was found that microscopically, after performing a topological duality twist 165], the effective two-dimensional theory of the D3-brane wrapping the curve preserves $\mathcal{N}=(0,4)$ supersymmetry and has left- and right-moving $S U(2)_{L, R}$ current algebras. A similar analysis was carried out in 166, where various topological duality twists of the D3brane worldvolume theory preserving $\mathcal{N}=(0, n)$ with $n=2,4,6,8$ supersymmetry are studied. A microscopic count of degrees of freedom of the worldvolume theory leads th

$$
\begin{aligned}
c_{L}^{\mathrm{flat}} & =3 C^{2}+9 c_{1}(B) \cdot C+2, \\
c_{R}^{\mathrm{flat}} & =6 k_{R}^{\mathrm{flat}}=3 C^{2}+3 c_{1}(B) \cdot C, \\
k_{L}^{\mathrm{flat}} & =\frac{1}{2} C^{2}-\frac{1}{2} c_{1}(B) \cdot C .
\end{aligned}
$$

The central charges and levels (3.88) corresponding to the asymptotically flat black string can be, up to the constants, reproduced from six-dimensional $(1,0)$ supergravity [76]. Ignoring the constants, the expressions (3.87) found in our setting reduce for $m=1$ to 3.88 . This nicely fits in the picture of the $4 \mathrm{D} / 5 \mathrm{D}$ correspondence 133, 134. However, the subleading terms in 3.88 that for the asymptotically flat case are higher derivative contributions, come in our case both from the two-derivative and the higher derivative part of the action.

\subsection{Discussion}

An alternative way to reproduce the central charges and levels computed in this chapter is by employing the effective action obtained by reducing M-theory on $\mathrm{CY}_{3}$. Upon further reduction on the near-horizon geometry $\mathrm{AdS}_{3} \times \mathrm{S}^{2}$ one can generate Chern-Simons terms, which in turn determine the central charges and right level [55]. The right-moving current algebra is identified with the $S U(2)$ isometry group of the sphere. Note that this $S U(2)$ is not the same as $S U(2)_{R}$ in our six-dimensional picture. Reproducing the left level from five dimensions works in a

\footnotetext{
${ }^{15}$ The center of mass contributions are subtracted.
} 
slightly different way, since the $U(1)_{L}$ corresponds to the circle of Taub-NUT that is now hidden in the geometry. However, one can do it using the data of the effective five-dimensional action in a similar way as we did in section 3.2 for the microscopic calculation. Although this was not our main motivation, in the six-dimensional approach the geometric realization of the left-moving current algebra is more clear.

In chapter 4 we will use the in this chapter further developed techniques to study more general black strings in F-theory. In particular we will replace the transverse Taub-NUT space by an arbitrary asymptotically locally Euclidean (ALE) or asymptotically locally flat (ALF) space. For these settings there is not always a controlled dual M-theory setting as we had in this chapter. Therefore, the macroscopic analysis needs to take place at the F-theory side in contrast to the setting in this chapter where there is an alternative via an M-theory supergravity analysis. Additionally we will examine the inclusion of vector multiplets and charged matter in the $6 \mathrm{~d}$ supergravity theory.

\section{Appendices}

\section{A $6 \mathrm{D}$ to $3 \mathrm{D}$ reduction higher derivative term}

In this appendix we give some more details of the reduction of the six-dimensional higher derivative term to three dimensions. In particular, we calculate the part of the integral

$$
\int_{\mathrm{TN}_{m}^{\mathrm{sph}}} \hat{G}^{\alpha} \wedge \hat{\omega}_{\mathrm{grav}}^{\mathrm{CS}}
$$

that leads to three-dimensional Chern-Simons terms. In order to do the reduction we first decompose the spin connection corresponding to the ansatz 3.52 to determine the parts that lead to Chern-Simons terms in three dimensions. Denoting indices of the non-spherical part $\mathcal{M}_{3}$ of the black string solution by $\tilde{a}=1,2,3$ and a vielbein of $\mathcal{M}_{3}$ by $\hat{e}^{\tilde{a}}$, the spin connection $\omega$ with respect to the vielbein of the ansatz, $e^{\tilde{a}} \equiv \hat{e}^{\tilde{a}}, e^{a}$, can be expressed as 167

$$
\begin{aligned}
\omega_{\tilde{a} \tilde{b}} & =\hat{\omega}_{\tilde{a} \tilde{b}}+\frac{1}{2} F_{\tilde{a} \tilde{b}}^{i} K_{c}^{i} e^{c}, \\
\omega_{\tilde{a} b} & =\frac{1}{2} F_{\tilde{a} \tilde{c}}^{i} K_{b}^{i} \hat{e}^{\tilde{c}}, \\
\omega_{a b} & =\hat{\omega}_{a b}+\left(\hat{\nabla}_{a} K_{b}^{i}\right) A^{i} .
\end{aligned}
$$

Here $\hat{\omega}_{\tilde{a} \tilde{b}}$ are the components of the spin connection $\hat{\omega}_{\mathcal{M}_{3}}$ with respect to the vielbein $\hat{e}^{\tilde{a}}$ of $\mathcal{M}_{3}$ and $\hat{\omega}_{a b}$ are the components of the spin connection $\hat{\omega}_{\mathrm{sph}}$ with 
respect to the vielbein $\hat{e}^{a}$ of the spherical part of the black string solution. From the expression of the gravitational Chern-Simons term,

$$
\hat{\omega}_{\mathrm{grav}}^{\mathrm{CS}}=\operatorname{tr}\left(\omega \wedge \mathrm{d} \omega+\frac{2}{3} \omega^{3}\right)
$$

it is immediately clear that if we are interested in three-dimensional Chern-Simons terms, we can restrict to

$$
\omega_{\tilde{a} \tilde{b}}=\hat{\omega}_{\tilde{a} \tilde{b}}, \quad \omega_{\tilde{a} b}=0, \quad \omega_{a b}=\hat{\omega}_{a b}+\left(\hat{\nabla}_{a} K_{b}^{i}\right) A^{i} .
$$

This is a direct sum connection, hence

$$
\hat{\omega}_{\mathrm{grav}}^{\mathrm{CS}}=\omega^{\mathrm{CS}}\left(\hat{\omega}_{\mathcal{M}_{3}}\right)+\omega^{\mathrm{CS}}(X),
$$

where $X$ is a connection with components $\hat{\omega}_{a b}+\left(\hat{\nabla}_{a} K_{b}^{i}\right) A^{i}$ and

$$
\begin{aligned}
\omega^{\mathrm{CS}}\left(\hat{\omega}_{\mathcal{M}_{3}}\right) & \equiv \operatorname{tr}\left(\hat{\omega}_{\mathcal{M}_{3}} \wedge \mathrm{d} \hat{\omega}_{\mathcal{M}_{3}}+\frac{2}{3} \hat{\omega}_{\mathcal{M}_{3}}^{3}\right), \\
\omega^{\mathrm{CS}}(X) & \equiv \operatorname{tr}\left(X \wedge \mathrm{d} X+\frac{2}{3} X^{3}\right) .
\end{aligned}
$$

Notice that $\omega^{\mathrm{CS}}\left(\hat{\omega}_{\mathcal{M}_{3}}\right)=\omega_{\text {grav }}^{\mathrm{CS}}$ is the gravitational Chern-Simons term of $\mathcal{M}_{3}$.

The only part of $\hat{G}^{\alpha}$ relevant for Chern-Simons terms in three dimensions is

$$
-Q^{\alpha}(2 \pi)^{2} m\left(e_{3}^{(m)}-\chi_{3}\right)
$$

Here $\chi_{3}$ has all its legs on $\mathcal{M}_{3}$ which means that its wedge product with $\hat{\omega}_{\text {grav }}^{\text {CS }}$ only gets a contribution of $\omega^{\mathrm{CS}}\left(\hat{\omega}_{\mathrm{sph}}\right)$. We can then expand

$$
\begin{aligned}
& \int_{\mathrm{TN}_{m}^{\mathrm{sph}}} \hat{G}^{\alpha} \wedge \hat{\omega}_{\mathrm{grav}}^{\mathrm{CS}}=-Q^{\alpha}(2 \pi)^{2} m \int_{\mathrm{TN}_{m}^{\mathrm{sph}}}\left[e_{3}^{(m)} \wedge \omega_{\mathrm{grav}}^{\mathrm{CS}}+e_{3}^{(m)} \wedge \omega^{\mathrm{CS}}(X)\right. \\
& \left.-\chi_{3} \wedge \omega^{\mathrm{CS}}\left(\hat{\omega}_{\mathrm{sph}}\right)\right] .
\end{aligned}
$$

The separate integrals are given by

$$
\begin{aligned}
\int_{\mathrm{TN}_{m}^{\mathrm{sph}}} e_{3}^{(m)} \wedge \omega_{\mathrm{grav}}^{\mathrm{CS}} & =-\frac{1}{m} \omega_{\mathrm{grav}}^{\mathrm{CS}}, \\
\int_{\mathrm{TN}_{m}^{\mathrm{sph}}} e_{3}^{(m)} \wedge \omega^{\mathrm{CS}}(X) & =\frac{2}{m} A_{L} \wedge F_{L}, \\
\int_{\mathrm{TN}_{m}^{\mathrm{sph}}} \chi_{3} \wedge \omega^{\mathrm{CS}}\left(\hat{\omega}_{\mathrm{sph}}\right) & =\frac{1+4 r^{\prime}+10 r^{\prime 2}+8 r^{\prime 3}+2 r^{\prime 4}}{m\left(1+r^{\prime}\right)^{4}} \times 16 \pi^{2} \chi_{3},
\end{aligned}
$$

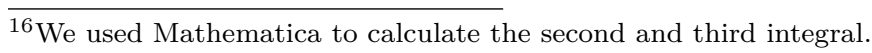


where we introduced $r^{\prime} \equiv m_{\infty} r / m$. Using that

$$
\chi_{3}=\frac{1}{16 \pi^{2}} A_{L} \wedge F_{L}+\frac{1}{8 \pi^{2}} \omega^{\mathrm{CS}}\left(A_{R}\right)
$$

we find that 3.96 becomes

$$
\begin{aligned}
\int_{\mathrm{TN}_{m}^{\mathrm{sph}}} \hat{G}^{\alpha} \wedge \hat{\omega}_{\mathrm{grav}}^{\mathrm{CS}}=Q^{\alpha}(2 \pi)^{2} & {\left[\omega_{\mathrm{grav}}^{\mathrm{CS}}-\frac{1+4 r^{\prime}+2 r^{\prime 2}}{\left(1+r^{\prime}\right)^{4}} A_{L} \wedge F_{L}\right.} \\
& \left.+2 \frac{1+4 r^{\prime}+10 r^{\prime 2}+8 r^{\prime 3}+2 r^{\prime 4}}{\left(1+r^{\prime}\right)^{4}} \omega^{\mathrm{CS}}\left(A_{R}\right)\right] .
\end{aligned}
$$

This leads to the expression (3.57).

\section{B Action of $\mathbb{Z}_{m}$ and $\mathbb{D}_{m}^{*}$ on $\mathrm{KK}$ spectrum}

In order to compute the one-loop quantum corrections for Taub-NUT as transverse spaces we must determine the spectrum on $\mathrm{AdS}_{3} \times \mathrm{S}^{3} / \mathbb{Z}_{m}$. This is achieved by taking the spectrum of $\mathrm{AdS}_{3} \times \mathrm{S}^{3}$, as given in 152,153 , and truncating out modes that are not invariant under $\mathbb{Z}_{m}$. In chapters 4 and 5 we also need to know what the spectrum is on $\mathrm{AdS}_{3} \times \mathrm{S}^{3} / \mathbb{D}_{m}^{*}$. Therefore, we shall consider the action of the two subgroups $\mathbb{Z}_{m}, \mathbb{D}_{m}^{*} \subset S U(2)_{L}$ in turn. The representations are determined by $S U(2)_{L}$ representations labeled by $j_{L}$ with $j_{L} \in \frac{1}{2} \mathbb{N}$ and with dimension $2 j_{L}+1$. The $j_{L}$ representation is given by the $2 j_{L}$-fold symmetrized tensor product of the fundamental representation $\left(j_{L}=\frac{1}{2}\right)$. The fundamental representation is given by the standard action of $S U(2)_{L}$ on $\mathbb{C}^{2}$. Let us denote the basis of $\mathbb{C}^{2}$ by

$$
|\uparrow\rangle=\left(\begin{array}{l}
1 \\
0
\end{array}\right), \quad|\downarrow\rangle=\left(\begin{array}{l}
0 \\
1
\end{array}\right) .
$$

The vector space $V_{j_{L}}$ corresponding to the representation labeled by $j_{L}$ is given by $V_{j_{L}}=\operatorname{Sym}^{2 j_{L}}\left(\mathbb{C}^{2}\right)$. A basis for this vector space is given by

$$
\begin{aligned}
\left|j_{L}, j_{L}^{3}\right\rangle & =\operatorname{Sym}^{2 j_{L}}\left(\uparrow^{j_{L}+j_{L}^{3}} \downarrow^{j_{L}-j_{L}^{3}}\right) \\
& \equiv \underbrace{|\uparrow\rangle \otimes \cdots \otimes|\uparrow\rangle}_{\left(j_{L}+j_{L}^{3}\right) \text { factors }} \otimes \underbrace{|\downarrow\rangle \otimes \cdots \otimes|\downarrow\rangle}_{\left(j_{L}-j_{L}^{3}\right) \text { factors }}+\text { symmetrized, }
\end{aligned}
$$

for $j_{L}^{3}=-j_{L},-j_{L}+1, \ldots, j_{L}$. Consider now the actions of $\mathbb{Z}_{m}$ and $\mathbb{D}_{m}^{*}$ on the above representations. 
Action $\mathbb{Z}_{\boldsymbol{m}}$. The action of the group $\mathbb{Z}_{m} \subset S U(2)_{L}$ on the fundamental representation is generated by

$$
\mathcal{A}=\left(\begin{array}{cc}
\mathrm{e}^{\frac{2 i \pi}{m}} & 0 \\
0 & \mathrm{e}^{-\frac{2 i \pi}{m}}
\end{array}\right) .
$$

Using this action and the construction of the other representations in terms of the fundamental one, we can explicitly deduce how the basis states $\left|j_{L}, j_{L}^{3}\right\rangle$ transform under the action of $\mathcal{A}$. We find

$$
\left|j_{L}, j_{L}^{3}\right\rangle \stackrel{\mathcal{A}}{\longrightarrow} \mathrm{e}^{4 \pi i \frac{j_{L}^{3}}{m}}\left|j_{L}, j_{L}^{3}\right\rangle
$$

The modes invariant under $\mathbb{Z}_{m}$ are those with $j_{L}^{3}=\frac{1}{2} m k$ for $k \in \mathbb{Z}$.

Action $\mathbb{D}_{\boldsymbol{m}}^{*}$. Consider now the action of $\mathbb{D}_{m}^{*} \subset S U(2)_{L}$ on the fundamental representation. It is generated by the two generators

$$
\mathcal{A}=\left(\begin{array}{cc}
\mathrm{e}^{\frac{i \pi}{m}} & 0 \\
0 & \mathrm{e}^{-\frac{i \pi}{m}}
\end{array}\right), \quad \mathcal{B}=\left(\begin{array}{cc}
0 & i \\
i & 0
\end{array}\right) .
$$

Similarly we find that under the action of the generator $\mathcal{A}$

$$
\left|j_{L}, j_{L}^{3}\right\rangle \stackrel{\mathcal{A}}{\longrightarrow} \mathrm{e}^{2 \pi i \frac{j_{L}^{3}}{m}}\left|j_{L}, j_{L}^{3}\right\rangle
$$

implying the projection condition $j_{L}^{3}=m k$ for $k \in \mathbb{Z}$. Note that this also implies $j_{L} \in \mathbb{N}$. For the generator $\mathcal{B}$ we find

$$
\left|j_{L}, j_{L}^{3}\right\rangle \stackrel{\mathcal{B}}{\longrightarrow}(-1)^{j_{L}}\left|j,-j_{L}^{3}\right\rangle
$$

To obtain invariant states under the generator $\mathcal{B}$ we construct the linear combinations

$$
\left|j_{L}, j_{L}^{3}\right\rangle+(-1)^{j_{L}}\left|j_{L},-j_{L}^{3}\right\rangle
$$

These states are then invariant under both generators provided $j_{L}^{3}=m k$ for $k \in \mathbb{Z}_{\geq 0}$.

\section{C $6 \mathrm{D}$ to $5 \mathrm{D}$ one-loop corrections}

In this appendix we use the index theorems to reproduce the results of [119] in which they calculated the one-loop corrections that one gets when integrating out massive chiral particles after the reduction from six to five dimensions on a circle. The gauge field that is relevant in this case is the $\mathfrak{u}(1)$ Kaluza-Klein vector $A^{0}$. We will calculate the contributions from spin- $\frac{1}{2}$ fermions, spin- $\frac{3}{2}$ fermions and for anti-symmetric tensors separately. 
Spin- $\frac{\mathbf{1}}{\mathbf{2}}$ fermions. We consider a massive spin- $\frac{1}{2}$ fermion coupled to the gauge field $A^{0}$ and to an external gravitational field denoted by the vielbein $e$. The parity anomaly resulting from this particle can be canceled by a term

$$
-i \pi \operatorname{sign}(M) \int_{\mathcal{M}_{5}} Q\left(A^{0}, \omega\right),
$$

where

$$
\mathrm{d} Q\left(A^{0}, \omega\right)=\hat{A}\left(\mathcal{M}_{5}\right) \wedge \operatorname{ch}\left(F^{0}\right) \mid
$$

Using that

$$
\hat{A}\left(\mathcal{M}_{5}\right) \wedge \operatorname{ch}\left(F^{0}\right) \mid=\frac{1}{(2 \pi)^{3}}\left(\frac{i^{3}}{6} F^{0} \wedge F^{0} \wedge F^{0}+\frac{i}{48} F^{0} \wedge \operatorname{tr} \mathcal{R} \wedge \mathcal{R}\right),
$$

we find that the counterterms should be given by

$$
\frac{\operatorname{sign}(M)}{8 \pi^{2}}\left(\frac{-1}{6} A^{0} \wedge F^{0} \wedge F^{0}+\frac{1}{48} A^{0} \wedge \operatorname{tr} \mathcal{R} \wedge \mathcal{R}\right) .
$$

Comparing conventions in 119 and 158 we find that we need $A^{0} \rightarrow q i A^{0}$ in the counterterm above. We also need to do a Wick rotation to obtain a Lorentzian action which gives another factor $i$. We thus find the counterterms

$$
\frac{\operatorname{sign}(M)}{8 \pi^{2}}\left(-\frac{q^{3}}{6} A^{0} \wedge F^{0} \wedge F^{0}-\frac{q}{48} A^{0} \wedge \operatorname{tr} \mathcal{R} \wedge \mathcal{R}\right)
$$

Spin- $\frac{\mathbf{3}}{\mathbf{2}}$ fermions. For spin- $\frac{3}{2}$ fermions the anomaly is canceled by a term of the form 3.108 where

$$
\begin{aligned}
\mathrm{d} Q(A, \omega) & =\hat{A}\left(\mathcal{M}_{5}\right) \wedge\left(\operatorname{tr} \mathrm{e}^{\mathrm{i} \mathcal{R} /(2 \pi)}-1\right) \wedge \operatorname{ch}\left(F^{0}\right) \mid \\
& =\frac{1}{(2 \pi)^{3}}\left(\frac{5 i^{3}}{6} F^{0} \wedge F^{0} \wedge F^{0}-\frac{19 i}{48} F^{0} \wedge \operatorname{tr} \mathcal{R} \wedge \mathcal{R}\right) .
\end{aligned}
$$

The counterterms to the Lorentzian action are thus given by

$$
\frac{\operatorname{sign}(M)}{8 \pi^{2}}\left(\frac{-5 q^{3}}{6} A^{0} \wedge F^{0} \wedge F^{0}+\frac{19 q}{48} A^{0} \wedge \operatorname{tr} \mathcal{R} \wedge \mathcal{R}\right) .
$$

Anti-symmetric tensors. In this case we were unable to find an index theorem in the literature, but following the arguments in the main text we postulate that the relevant index is given by

$$
\text { ind } i D_{A}=\frac{1}{2} \int L(M) \wedge \operatorname{ch}\left(2 F^{0}\right) \mid \text {. }
$$


Since the tensors are bosons, the counterterm is now given by 159

$$
i \pi \operatorname{sign}(M) \int_{\mathcal{M}_{5}} Q\left(A^{0}, \omega\right),
$$

where

$$
\begin{aligned}
\mathrm{d} Q\left(A^{0}, \omega\right) & =\frac{1}{2} L(M) \wedge \operatorname{ch}\left(2 F^{0}\right) \mid \\
& =\frac{1}{8 \pi^{3}}\left(\frac{2 i^{3}}{3} F^{0} \wedge F^{0} \wedge F^{0}-\frac{i}{6} F^{0} \wedge \operatorname{tr} \mathcal{R} \wedge \mathcal{R}\right) .
\end{aligned}
$$

This implies that the counterterms to the Lorentzian action are

$$
\frac{\operatorname{sign}(M)}{8 \pi^{2}}\left(\frac{2 q^{3}}{3} A^{0} \wedge F^{0} \wedge F^{0}-\frac{q}{6} A^{0} \wedge \operatorname{tr} \mathcal{R} \wedge \mathcal{R}\right) .
$$

The terms (3.112), 3.114 and (3.118) are precisely the one-loop contributions of table 2.2 in 119 .

\section{D Summation of 6D to 3D one-loop corrections}

To perform the sum of the one-loop corrections over the Kaluza-Klein spectrum, we use the regularization procedure described in the main text. We need the sums

$$
\begin{aligned}
& \sum_{j_{L}=\frac{1}{2} m k}^{\infty} 1=\frac{1}{2}-\frac{1}{2} k m, \quad \sum_{j_{L}=\frac{1}{2} m k}^{\infty} j_{L}=\frac{1}{24}\left(-2+6 k m-3 k^{2} m^{2}\right) \\
& \sum_{j_{L}=\frac{1}{2} m k}^{\infty} j_{L}^{2}=\frac{1}{24}\left(-2 k m+3 k^{2} m^{2}-k^{3} m^{3}\right)
\end{aligned}
$$

where the sum is over integers (half integers) when $\frac{1}{2} m k$ is integer (half integer). The sums used in this section can then be calculated using (3.83):

$$
\begin{array}{rlrl}
\sum_{k=1}^{\infty} \sum_{j_{L}=\frac{1}{2} m k}^{\infty} 1=-\frac{1}{4}+\frac{1}{24} m, & & \sum_{k=1}^{\infty} \sum_{j_{L}=\frac{1}{2} m k}^{\infty} j_{L}=\frac{1}{24}-\frac{1}{48} m, \\
\sum_{k=1}^{\infty} \sum_{j_{L}=\frac{1}{2} m k}^{\infty} j_{L}^{2}=-\frac{m^{3}}{24 \cdot 120}+\frac{m}{144}, & \sum_{k=1}^{\infty} \sum_{j_{L}=\frac{1}{2} m k}^{\infty} k^{2}=-\frac{1}{2} \frac{m}{120} .
\end{array}
$$

We calculate the corrections to the levels $k_{L}^{\text {loop }}, k_{R}^{\text {loop }}$ and the correction to the difference of the central charges $\left(c_{L}-c_{R}\right)^{\text {loop }}$ separately. Using the projection 
condition $j_{L}^{3}=\frac{1}{2} m k$, we calculate each time first the contribution of the $k=0$ representations and after that the contribution of $k \neq 0$. Since the structure of representations for small values of $j_{L}, j_{R}$ becomes more complicated, we first calculate the corrections for $m \geq 3$ and do the cases $m=1,2$ separately.

\section{D.1 Corrections for $m \geq 3$}

We list the $k=0$ representations where all the sums are over integers

- Spin- $\frac{3}{2}$ :

$$
2 \bigoplus_{j_{L}=1}^{\infty}\left(j_{L}, j_{L} \pm \frac{1}{2}\right)^{\mp}
$$

- Spin- $\frac{1}{2}$ :

$$
\begin{gathered}
2 \bigoplus_{j_{L}=2}^{\infty}\left(j_{L}, j_{L} \pm \frac{3}{2}\right)^{\mp} \oplus 2 \bigoplus_{j_{L}=0}^{1}\left(j_{L}, j_{L}+\frac{3}{2}\right)^{-} \oplus 2 \bigoplus_{j_{L}=1}^{\infty}\left(j_{L}, j_{L} \pm \frac{1}{2}\right)^{ \pm} \\
\oplus 2\left(0, \frac{1}{2}\right)^{+} \oplus 2\left(n_{T}+n_{H}\right) \bigoplus_{j_{L}=1}^{\infty}\left(j_{L}, j_{L} \pm \frac{1}{2}\right)^{ \pm} \oplus 2\left(n_{T}+n_{H}\right)\left(0, \frac{1}{2}\right)^{+} .
\end{gathered}
$$

- vectors:

$$
\bigoplus_{j_{L}=1}^{\infty}\left(j_{L}, j_{L} \pm 1\right)^{\mp} \oplus(0,1)^{-} \oplus n_{T} \bigoplus_{j_{L}=1}^{\infty}\left(j_{L}, j_{L} \pm 1\right)^{ \pm} \oplus n_{T}(0,1)^{+}
$$

Note that the $(0,1)^{-} \oplus n_{T}(0,1)^{+}$vector representations are mapped to itself when applying the reality condition. Hence their contribution comes with an extra factor $\frac{1}{2}$.

When $k>0$ the projection condition gives that $j_{L}^{3}=\frac{1}{2} m k \geq \frac{m}{2}$ which means that when $m \geq 3$ we only need the representations (again the sums go with integer steps)

- Spin- $\frac{3}{2}$ :

$$
2 \bigoplus_{j_{L}=\frac{1}{2} m k}^{\infty}\left(j_{L}, j_{L} \pm \frac{1}{2}\right)^{\mp}
$$


- Spin- $\frac{1}{2}$ :

$$
\begin{array}{r}
2 \bigoplus_{j_{L}=\frac{1}{2} m k}^{\infty}\left(j_{L}, j_{L} \pm \frac{3}{2}\right)^{\mp} \oplus 2 \bigoplus_{j_{L}=\frac{1}{2} m k}^{\infty}\left(j_{L}, j_{L} \pm \frac{1}{2}\right)^{ \pm} \\
\oplus 2\left(n_{T}+n_{H}\right) \bigoplus_{j_{L}=\frac{1}{2} m k}^{\infty}\left(j_{L}, j_{L} \pm \frac{1}{2}\right)^{ \pm} .
\end{array}
$$

- vectors:

$$
\bigoplus_{j_{L}=\frac{1}{2} m k}^{\infty}\left(j_{L}, j_{L} \pm 1\right)^{\mp} \oplus n_{T} \bigoplus_{j_{L}=\frac{1}{2} m k}^{\infty}\left(j_{L}, j_{L} \pm 1\right)^{ \pm}
$$

Correction to left level. In this case we do not have a contribution of the $k=0$ modes. We thus only have to calculate the $k>0$ modes and we will do this separately for the various types of fields contributing to the left level. For the spin- $\frac{3}{2}$ fermions we get

$$
\begin{aligned}
\alpha_{L}^{(3 / 2)} & =2 \sum_{k=1}^{\infty} \sum_{j_{L}=\frac{1}{2} m k}^{\infty} \frac{3}{8 \pi}\left(\frac{1}{2} m k\right)^{2}\left[2\left(j_{L}-\frac{1}{2}\right)+1-2\left(j_{L}+\frac{1}{2}\right)-1\right] \\
& =-\frac{3 m^{2}}{8 \pi} \sum_{k=1}^{\infty} \sum_{j=\frac{1}{2} m k}^{\infty} k^{2}=\frac{1}{8 \pi} \frac{m^{3}}{80} .
\end{aligned}
$$

In the same way the spin- $\frac{1}{2}$ fermions give

$$
\begin{aligned}
\alpha_{L}^{(1 / 2)} & =\frac{1}{8 \pi} \sum_{k=1}^{\infty} \sum_{j_{L}=\frac{1}{2} m k}^{\infty}\left[-12\left(\frac{1}{2} m k\right)^{2}+4\left(\frac{1}{2} m k\right)^{2}+4\left(n_{T}+n_{H}\right)\left(\frac{1}{2} m k\right)^{2}\right] \\
& =-\frac{m^{2}}{8 \pi}\left(2-n_{T}-n_{H}\right) \sum_{k=1}^{\infty} \sum_{j_{L}=\frac{1}{2} m k}^{\infty} k^{2}=\frac{1}{8 \pi} \frac{m^{3}}{240}\left(2-n_{T}-n_{H}\right) .
\end{aligned}
$$

The vectors contribute with

$$
\alpha_{L}^{(\text {vect })}=4\left(1-n_{T}\right) \sum_{k=1}^{\infty} \sum_{j_{L}=\frac{1}{2} m k}^{\infty} \frac{1}{4 \pi}\left(\frac{1}{2} m k\right)^{2}=-\frac{1}{8 \pi} \frac{m^{3}}{120}\left(1-n_{T}\right) .
$$

Adding the contributions $3.121,3.122$ and 3.123 , we find

$$
k_{L}^{\text {loop }}=8 \pi \cdot\left(\alpha_{L}^{(3 / 2)}+\alpha_{L}^{(1 / 2)}+\alpha_{L}^{(\text {vect })}\right)=-\frac{m^{3}}{8} c_{1}(B)^{2},
$$

where we used the identities (3.84). 
Correction to right level. We get the contribution of the $k=0$ modes by summing over the representations listed above. We first do this for the $2\left(j_{L}, j_{L} \pm \frac{1}{2}\right) \mp$ representations for the spin- $\frac{3}{2}$ fermions, the $2\left(j_{L}, j_{L} \pm \frac{3}{2}\right) \mp$ representations for the spin- $\frac{1}{2}$ fermions and the $\left(1-n_{T}\right)\left(j_{L}, j_{L} \pm 1\right)^{\mp}$ representations for the vectors, which are in this order given by

$$
\begin{array}{r}
-\frac{1}{2 \pi} \sum_{j_{L}=1}^{\infty}\left[-\left(j_{L}+\frac{1}{2}\right)\left(j_{L}+\frac{3}{2}\right)\left(2 j_{L}+2\right)+\left(j_{L}-\frac{1}{2}\right)\left(j_{L}+\frac{1}{2}\right)\left(2 j_{L}\right)\right]=-\frac{5}{8 \pi}, \\
-\frac{1}{6 \pi} \sum_{j_{L}=2}^{\infty}\left[-\left(j_{L}+\frac{3}{2}\right)\left(j_{L}+\frac{5}{2}\right)\left(2 j_{L}+4\right)+\left(j_{L}-\frac{3}{2}\right)\left(j_{L}-\frac{1}{2}\right)\left(2 j_{L}-2\right)\right]=-\frac{83}{8 \pi},
\end{array}
$$

and

$$
\begin{aligned}
\frac{1}{6 \pi}\left(1-n_{T}\right) \sum_{j_{L}=1}^{\infty}\left[-\left(j_{L}+1\right)\right. & \left(j_{L}+2\right)\left(2 j_{L}+3\right) \\
& \left.+\left(j_{L}-1\right) j_{L}\left(2 j_{L}-1\right)\right]=\frac{2}{3 \pi}\left(1-n_{T}\right) .
\end{aligned}
$$

For the spin- $\frac{1}{2}$ fields we then also need to sum over the other infinite towers of states, namely the $2\left(j_{L}, j_{L} \pm \frac{1}{2}\right)^{ \pm}$and $2\left(n_{T}+n_{H}\right)\left(j_{L}, j_{L} \pm \frac{3}{2}\right) \mp$ representations. These can be determined by inserting the right relative factors in the first of the sums above. We also add the contributions from the isolated representations, which are not part of an infinite tower in the spectrum. These are in the case of spin- $\frac{1}{2}$ fields the $2\left(0, \frac{3}{2}\right)^{-} \oplus 2\left(1, \frac{5}{2}\right)^{-} \oplus 2\left(n_{T}+n_{H}+1\right)\left(0, \frac{1}{2}\right)^{+}$representations. Their contribution is given by

$$
\frac{5}{12 \pi}-\left(n_{T}+n_{H}\right) \frac{11}{24 \pi}
$$

Lastly, we need to sum over the isolated $(0,1)^{-} \oplus n_{T}(0,1)^{+}$representations for the vectors. Since they are mapped to itself when applying the reality condition, we have to add an extra factor $\frac{1}{2}$. This results in

$$
-\frac{1-n_{T}}{2 \pi}
$$

Summing all the different contributions gives

$$
\alpha_{R}^{k=0}=\frac{1}{8 \pi}\left(3-\frac{1}{3} n_{H}-\frac{5}{3} n_{T}\right) .
$$


We calculate the $k \neq 0$ contributions in the same way as for the left level. The spin- $\frac{3}{2}$ fermions contribute

$$
\begin{aligned}
\alpha_{R}^{(3 / 2)} & =-\frac{1}{2 \pi} \sum_{k=1}^{\infty} \sum_{j_{L}=\frac{1}{2} m k}^{\infty}\left[-\left(j_{L}+\frac{1}{2}\right)\left(j_{L}+\frac{3}{2}\right)\left(2 j_{L}+2\right)+\left(j_{L}-\frac{1}{2}\right)\left(j_{L}+\frac{1}{2}\right) 2 j_{L}\right] \\
& =\frac{1}{2 \pi} \sum_{k=1}^{\infty} \sum_{j_{L}=\frac{1}{2} m k}^{\infty}\left(\frac{3}{2}+6 j_{L}+6 j_{L}^{2}\right)=\frac{1}{4 \pi}\left(-\frac{1}{4}-\frac{m}{24}-\frac{m^{3}}{240}\right) .
\end{aligned}
$$

We calculate the contribution of the spin- $\frac{1}{2}$ fermions by first summing over the $\left(j_{L}, j_{L} \pm \frac{3}{2}\right)^{\mp}$ representations

$$
\begin{aligned}
\alpha_{R}^{(1 / 2)}= & -\frac{1}{6 \pi} \sum_{k=1}^{\infty} \sum_{j_{L}=\frac{1}{2} m k}^{\infty}\left[-\left(j_{L}+\frac{3}{2}\right)\left(j_{L}+\frac{5}{2}\right)\left(2 j_{L}+4\right)\right. \\
& \left.+\left(j_{L}-\frac{3}{2}\right)\left(j_{L}-\frac{1}{2}\right)\left(2 j_{L}-2\right)\right] \\
& -\frac{1}{6 \pi}\left(1+n_{T}+n_{H}\right) \sum_{k=1}^{\infty} \sum_{j_{L}=\frac{1}{2} m k}^{\infty}\left[\left(j_{L}+\frac{1}{2}\right)\left(j_{L}+\frac{3}{2}\right)\left(2 j_{L}+2\right)\right. \\
= & \left.\frac{1}{4 \pi}\left(-\frac{9}{4}+\frac{7 m}{24}-\frac{m^{3}}{240}\right)-\frac{1}{4 \pi}\left(1+n_{T}+n_{H}\right)\left(-\frac{1}{2}\right)\left(j_{L}+\frac{1}{2}\right) 2 j_{L}\right]
\end{aligned}
$$

Lastly, the vectors give

$$
\begin{aligned}
\alpha_{R}^{\text {(vect) }}= & \frac{1}{6 \pi}\left(1-n_{T}\right) \sum_{k=1}^{\infty} \sum_{j_{L}=\frac{1}{2} m k}^{\infty}\left[-\left(j_{L}+1\right)\left(j_{L}+2\right)\left(2 j_{L}+3\right)\right. \\
& \left.+\left(j_{L}-1\right) j_{L}\left(2 j_{L}-1\right)\right] \\
= & -\frac{1}{6 \pi}\left(1-n_{T}\right) \sum_{k=1}^{\infty} \sum_{j_{L}=\frac{1}{2} m k}^{\infty}\left(6+12 j_{L}+12 j_{L}^{2}\right) \\
= & \frac{1}{4 \pi}\left(n_{T}-1\right)\left(-\frac{2}{3}+\frac{m}{18}-\frac{m^{3}}{360}\right) .
\end{aligned}
$$

Adding the contributions 3.129, 3.130, 3.131 and 3.132, we get the following correction to the right level

$$
\begin{aligned}
k_{R}^{\text {loop }} & =4 \pi\left[\alpha_{R}^{k=0}+\alpha_{R}^{(3 / 2)}+\alpha_{R}^{(1 / 2)}+\alpha_{R}^{(\text {vect })}\right] \\
& =\frac{m^{3}}{24} c_{1}(B)^{2}+\frac{m}{3} c_{1}(B)^{2}+m-c_{1}(B)^{2}-14 .
\end{aligned}
$$


Correction to $c_{\boldsymbol{L}}-\boldsymbol{c}_{\boldsymbol{R}}$. We do these calculations in the same way as before. We first calculate the contribution of the $k=0$ states. Summing over these states in the spin- $\frac{1}{2}$, spin- $\frac{3}{2}$ and vector spectrum results in

$$
\begin{aligned}
\alpha_{\text {grav }}^{k=0} & =\frac{1}{192 \pi}\left[-12 \sum_{j_{L}=2}^{\infty} 1+4\left(1+n_{T}+n_{H}\right) \sum_{j_{L}=1}^{\infty} 1-2 \cdot 4+2 \cdot 2+2\left(n_{H}+n_{T}\right) \cdot 2\right. \\
& -2 \cdot 6]+4 \sum_{j_{L}=1}^{\infty} \frac{7}{64 \pi}-4\left(1-n_{T}\right) \sum_{j_{L}=1}^{\infty} \frac{1}{48 \pi}-\frac{1}{2} \cdot \frac{1}{48 \pi}\left(1-n_{T}\right) \cdot 3 \\
& =\frac{1}{96 \pi}\left(n_{H}+n_{T}\right)-\frac{7}{32 \pi}+\frac{1}{96 \pi}\left(1-n_{T}\right)=-\frac{1}{96 \pi}\left(20-n_{H}\right) .
\end{aligned}
$$

We now calculate the contribution of the $k \neq 0$ representations. Spin- $\frac{3}{2}$ fermions give the following correction

$$
\alpha_{\text {grav }}^{(3 / 2)}=\frac{7}{16 \pi} \sum_{k=1}^{\infty} \sum_{j_{L}=\frac{1}{2} m k}^{\infty} 1=\frac{7}{16 \pi}\left(-\frac{1}{4}+\frac{1}{24} m\right)=\frac{1}{96 \pi}\left(-\frac{21}{2}+\frac{7}{4} m\right) .
$$

From spin- $\frac{1}{2}$ fermions we obtain

$$
\begin{aligned}
\alpha_{\text {grav }}^{(1 / 2)} & =-\frac{1}{4 \cdot 48 \pi} \sum_{k=1}^{\infty} \sum_{j_{L}=\frac{1}{2} m k}^{\infty}\left[2 \cdot 6-2 \cdot 2-2\left(n_{T}+n_{H}\right) \cdot 2\right] \\
& =-\frac{1}{96 \pi}\left(4-2\left(n_{T}+n_{H}\right)\right) \sum_{k=1}^{\infty} \sum_{j=\frac{1}{2} m k}^{\infty} 1 \\
& =-\frac{1}{96 \pi}\left(4-2\left(n_{T}+n_{H}\right)\right)\left(-\frac{1}{4}+\frac{1}{24} m\right) .
\end{aligned}
$$

Finally, for the vectors we find

$$
\alpha_{\mathrm{grav}}^{(\mathrm{vect})}=-\frac{1}{48 \pi}\left(1-n_{T}\right) \sum_{k=1}^{\infty} \sum_{j_{L}=\frac{1}{2} m k}^{\infty} 4=-\frac{1}{96 \pi}\left(1-n_{T}\right)\left(-2+\frac{m}{3}\right) .
$$

Adding the results 3.134), 3.135, 3.136) and 3.137) yields the following correction to $c_{L}-c_{R}$

$$
\begin{aligned}
\left(c_{L}-c_{R}\right)^{\text {loop }} & =96 \pi \cdot\left(\alpha_{\mathrm{grav}}^{k=0}+\alpha_{\mathrm{grav}}^{(3 / 2)}+\alpha_{\mathrm{grav}}^{(1 / 2)}+\alpha_{\mathrm{grav}}^{(\mathrm{vect})}\right) \\
& =6 m+(2 m+17) c_{1}(B)^{2}-44 .
\end{aligned}
$$




\section{D.2 Corrections for $m=2$}

For $m=2$ the representations we need to take into account for $k=0$ stay the same. For $k>0$ only the spin- $\frac{1}{2}$ contribution changes. Summing the correct representations in the spin- $\frac{1}{2}$ sector, we again find the one-loop corrections.

Correction to left level. For the spin- $\frac{1}{2}$ fermions we now find

$$
\begin{aligned}
\alpha_{L}^{(1 / 2)}= & -12 \sum_{k=2}^{\infty} \sum_{j_{L}=k}^{\infty} \frac{1}{8 \pi} k^{2}-12 \sum_{j_{L}=2}^{\infty} \frac{1}{8 \pi}-12 \frac{1}{8 \pi}+4 \sum_{k=1}^{\infty} \sum_{j_{L}=k}^{\infty} \frac{1}{8 \pi} k^{2} \\
& +4\left(n_{T}+n_{H}\right) \sum_{k=1}^{\infty} \sum_{j_{L}=k}^{\infty} \frac{1}{8 \pi} k^{2}=\frac{1}{120 \pi}-\frac{n_{T}+n_{H}}{240 \pi}
\end{aligned}
$$

which is exactly the same as 3.122 for $m=2$.

Correction to right level. The change in contribution of the spin- $\frac{1}{2}$ fermions is caused by the $\left(j_{L}, j_{L} \pm \frac{3}{2}\right)^{\mp} \oplus 2\left(1, \frac{5}{2}\right)^{-}$representations. Its contribution is given by

$$
\begin{aligned}
& -\frac{1}{6 \pi} \sum_{k=2}^{\infty} \sum_{j_{L}=k}^{\infty}\left[-\left(j_{L}+\frac{3}{2}\right)\left(j_{L}+\frac{5}{2}\right)\left(2 j_{L}+4\right)+\left(j_{L}-\frac{3}{2}\right)\left(j_{L}-\frac{1}{2}\right)\left(2 j_{L}-2\right)\right] \\
& -\frac{1}{6 \pi} \sum_{j_{L}=2}^{\infty}\left[-\left(j_{L}+\frac{3}{2}\right)\left(j_{L}+\frac{5}{2}\right)\left(2 j_{L}+4\right)+\left(j_{L}-\frac{3}{2}\right)\left(j_{L}-\frac{1}{2}\right)\left(2 j_{L}-2\right)\right]+\frac{35}{4 \pi},
\end{aligned}
$$

which when combined with the other spin- $\frac{1}{2}$ representations (for which the summation goes the same as in the $m \geq 3$ case) gives

$$
\alpha_{R}^{(1 / 2)}=-\frac{71}{180 \pi}+\frac{11\left(n_{H}+n_{T}\right)}{360 \pi} .
$$

This is again the same as the contribution 3.131 for $m=2$. 
Correction to $c_{\boldsymbol{L}}-c_{\boldsymbol{R}}$. This time, we find the following contribution for the spin- $\frac{1}{2}$ fermions:

$$
\begin{aligned}
\alpha_{\text {grav }}^{(1 / 2)}=-\frac{1}{4 \cdot 48 \pi}\left[2 \sum_{k=2}^{\infty} \sum_{j_{L}=k}^{\infty} 6+2 \sum_{j_{L}=2}^{\infty} 6+12\right. \\
\left.\quad-2 \sum_{k=1}^{\infty} \sum_{j_{L}=k}^{\infty} 2-2\left(n_{T}+n_{H}\right) \sum_{k=1}^{\infty} \sum_{j_{L}=k}^{\infty} 2\right] \\
=\frac{1}{144 \pi}-\frac{n_{T}+n_{H}}{288 \pi},
\end{aligned}
$$

which is the same as 3.136 for $m=2$.

\section{D.3 Corrections for $m=1$}

The change in summations is again only for $k>0$, but in this case it is both in the spin- $\frac{1}{2}$ sector and in the vector sector.

Correction to left level. For the spin- $\frac{1}{2}$ fermions we find

$$
\begin{aligned}
\alpha_{L}^{(1 / 2)}= & -12 \sum_{k=3}^{\infty} \sum_{j_{L}=\frac{1}{2} k}^{\infty} \frac{1}{8 \pi}\left(\frac{1}{2} k\right)^{2}-12 \sum_{j_{L}=2}^{\infty} \frac{1}{8 \pi}-12 \sum_{j_{L}=\frac{3}{2}}^{\infty} \frac{1}{8 \pi}\left(\frac{1}{2}\right)^{2}-\frac{3}{2 \pi} \\
& -10 \frac{1}{8 \pi}\left(\frac{1}{2}\right)^{2}+4 \sum_{k=2}^{\infty} \sum_{j_{L}=\frac{1}{2} k}^{\infty} \frac{1}{8 \pi}\left(\frac{1}{2} k\right)^{2}+4 \sum_{j_{L}=3 / 2}^{\infty} \frac{1}{8 \pi}\left(\frac{1}{2}\right)^{2} \\
& +6 \frac{1}{8 \pi}\left(\frac{1}{2}\right)^{2}+4\left(n_{T}+n_{H}\right) \sum_{k=1}^{\infty} \sum_{j_{L}=\frac{1}{2} k}^{\infty} \frac{1}{8 \pi}\left(\frac{1}{2} k\right)^{2}=\frac{121}{960 \pi}-\frac{n_{H}+n_{T}}{1920 \pi},
\end{aligned}
$$

which is not the same as 3.122 for $m=1$. The vector contribution changes to

$$
\begin{aligned}
\alpha_{L}^{(\text {vect })}= & 4\left(1-n_{T}\right) \sum_{k=2}^{\infty} \sum_{j_{L}=\frac{1}{2} k}^{\infty} \frac{1}{4 \pi}\left(\frac{1}{2} k\right)^{2} \\
& +4\left(1-n_{T}\right) \sum_{j_{L}=\frac{3}{2}}^{\infty} \frac{1}{4 \pi} \cdot \frac{1}{4}+4\left(1-n_{T}\right) \frac{1}{4 \pi} \cdot \frac{1}{4} \\
= & -\frac{1-n_{T}}{960 \pi},
\end{aligned}
$$

which is exactly the same as 3.123 for $m=1$. 
Correction to right level. We first calculate the contribution of the fermions with spin- $\frac{1}{2}$. For the $2 \bigoplus_{j_{L}=\frac{3}{2}}^{\infty}\left(j_{L}, j_{L} \pm \frac{3}{2}\right)^{\mp}$ representations we find

$$
\begin{aligned}
-\frac{1}{6 \pi}\left[\sum_{k=3}^{\infty} \sum_{j_{L}=k / 2}^{\infty}+\sum_{j_{L}=2}^{\infty}+\sum_{j_{L}=3 / 2}^{\infty}\right][ & -\left(j_{L}+\frac{3}{2}\right)\left(j_{L}+\frac{5}{2}\right)\left(2 j_{L}+4\right) \\
& \left.+\left(j_{L}-\frac{3}{2}\right)\left(j_{L}-\frac{1}{2}\right)\left(2 j_{L}-2\right)\right]=-\frac{4557}{320 \pi} .
\end{aligned}
$$

The $2 \bigoplus_{j_{L}=1}^{\infty}\left(j_{L}, j_{L} \pm \frac{1}{2}\right)^{ \pm}$representations give

$$
\begin{aligned}
-\frac{1}{6 \pi}\left[\sum_{k=2}^{\infty} \sum_{j_{L}=k / 2}^{\infty}+\sum_{j_{L}=3 / 2}^{\infty}\right]\left[-\left(j_{L}\right.\right. & \left.+\frac{1}{2}\right)\left(j_{L}+\frac{3}{2}\right)\left(2 j_{L}+2\right) \\
& \left.+\left(j_{L}-\frac{1}{2}\right)\left(j_{L}+\frac{1}{2}\right)\left(2 j_{L}\right)\right]=\frac{2951}{2880 \pi} .
\end{aligned}
$$

For the $2 \bigoplus_{j_{L}=0}^{1}\left(j_{L}, j_{L}+\frac{3}{2}\right)^{-} \oplus 2\left(\frac{1}{2}, 1\right)^{+}$representations we find a contribution

$$
-\frac{1}{6 \pi}\left(-\frac{5}{2} \cdot \frac{7}{2} \cdot 6-2 \cdot 3 \cdot 5+1 \cdot 2 \cdot 3\right)=\frac{51}{4 \pi}
$$

The contribution of the $2\left(n_{T}+n_{H}\right) \bigoplus_{j_{L}=\frac{1}{2}}^{\infty}\left(j_{L}, j_{L} \pm \frac{1}{2}\right)^{ \pm}$representations stays the same. Adding all the contributions we find

$$
\alpha_{R}^{(1 / 2)}=-\frac{671}{1440 \pi}+\left(n_{H}+n_{T}\right) \frac{71}{2880 \pi}
$$

which is the same as 3.131 for $m=1$. The vector contribution changes to

$$
\begin{aligned}
\alpha_{R}^{(\text {vect })}= & \frac{1}{6 \pi}\left(1-n_{T}\right) \sum_{k=2}^{\infty} \sum_{j_{L}=\frac{1}{2} k}^{\infty}\left[-\left(j_{L}+1\right)\left(j_{L}+2\right)\left(2 j_{L}+3\right)\right. \\
& \left.+\left(j_{L}-1\right) j_{L}\left(2 j_{L}-1\right)\right] \\
& +\frac{1}{6 \pi}\left(1-n_{T}\right) \sum_{j_{L}=\frac{3}{2}}^{\infty}\left[-\left(j_{L}+1\right)\left(j_{L}+2\right)\left(2 j_{L}+3\right)+\left(j_{L}-1\right) j_{L}\left(2 j_{L}-1\right)\right] \\
= & \left(1-n_{T}\right) \frac{221}{1440 \pi},
\end{aligned}
$$

which is the same as 3.132 for $m=1$. 
Correction to $c_{\boldsymbol{L}}-c_{\boldsymbol{R}}$. Now we find for the spin- $\frac{1}{2}$ fields

$$
\begin{aligned}
\alpha_{\text {grav }}^{(1 / 2)=} \frac{1}{4 \cdot 48 \pi}[ & -2 \sum_{k=3}^{\infty} \sum_{j_{L}=k / 2}^{\infty} 6-2 \sum_{j_{L}=2}^{\infty} 6-2 \sum_{j_{L}=3 / 2}^{\infty} 6-12-10 \\
& \left.+2 \sum_{k=2}^{\infty} \sum_{j_{L}=k / 2}^{\infty} 2+2 \sum_{j_{L}=3 / 2}^{\infty} 2+6-2\left(n_{T}+n_{H}\right) \sum_{k=1}^{\infty} \sum_{j_{L}=k / 2}^{\infty} 2\right] \\
= & \frac{17}{576 \pi}-\frac{5}{1152 \pi}\left(n_{T}+n_{H}\right) .
\end{aligned}
$$

For the vectors we get

$$
\alpha_{\text {grav }}^{\text {(vect) }}=-\frac{1}{12 \pi}\left(1-n_{T}\right)\left[\sum_{k=2}^{\infty} \sum_{j_{L}=\frac{1}{2} k}^{\infty} 1+\sum_{j_{L}=3 / 2}^{\infty} 1+1\right]=\frac{5}{288 \pi}-\frac{5}{288 \pi} n_{T},
$$

which is the same as 3.137 for $m=1$. 


\section{Black strings in F-theory with ALE/ALF spaces transversely}

In the preceding chapter we have studied black strings in F-theory compactified on an elliptically fibered Calabi-Yau threefold. The black strings arose from D3-branes wrapped on a curve in the base of the fibration. We took a Taub-NUT as transverse space which is a circle fibration over $\mathbb{R}^{3}$ such that this setting corresponds to four-dimensional black holes. Microscopically, this system is described by a $2 \mathrm{~d}$ $\mathcal{N}=(0,4)$ superconformal field theory (SCFT) which is the IR fixed point of the D3-brane worldvolume theory compactified on a curve. The entropy corresponding to the black string is via the Cardy formula expressed in terms of the central charges and levels of the SCFT. In chapter 3 we have computed these numbers by reducing the six-dimensional $\mathcal{N}=(1,0)$ supergravity theory on the spherical part of the black string geometry. The central charges and levels correspond to the coefficients of Chern-Simons terms in the resulting $3 \mathrm{~d}$ action. We have matched these to the microscopic values.

In this chapter, which is based on [5], we will generalize the setting of chapter 3 in two ways. Firstly, we replace the transverse Taub-NUT space by a general asymptotically locally Euclidean (ALE) or asymptotically locally flat (ALF) space. Taub-NUT is an example of the latter. Secondly, in chapter 3 we have only considered Calabi-Yau threefolds in which the fiber degenerations left the whole fibration non-singular. This results in six-dimensional supergravity theories without vector multiplets. In this chapter we will however also study settings with vector multiplets.

\subsection{Introduction}

In this chapter we consider D3-branes in an asymptotic F-theory geometry $\mathbb{R} \times$ $\mathrm{S}^{1} \times M \times \mathrm{CY}_{3}$ where the D3-branes are wrapped on $\mathrm{S}^{1} \times C$ with $C \subset B$ a curve in the base of the elliptically fibered $\mathrm{CY}_{3}$ and probe the transverse space $M$. The equations of motion and supersymmetry preservation imply that the four-manifold $M$ is a Ricci-flat hyper-Kähler manifold which we take to be non-compact. We 
consider two families of such manifolds $M=M_{\Gamma}$ : asymptotically locally Euclidean (ALE) and asymptotically locally flat (ALF) spaces, both of which may be defined by a choice of discrete subgroup $\Gamma \subset S U(2)$ as we review in section 4.2 . In the preceding chapter 3 we have studied the case where $M_{\Gamma}=\mathrm{TN}_{m}$, i.e. $\mathrm{ALF}$ and $\Gamma$ corresponding to the A-series within the ADE-classification of discrete subgroups of $S U(2)$. Other choices of $\Gamma$ or taking $M_{\Gamma}$ ALE lead to black strings with different charges. Microscopically the strings are dual to $2 \mathrm{~d} \mathcal{N}=(0,4)$ SCFTs which admit both left- and right-moving central charges $c_{L, R}$. In addition they always admit a right-moving $S U(2)_{R}$ current algebra and in the case of the A-series also a leftmoving $U(1)_{L}$ current algebra, each with an associated level. In sections 4.3 and 4.4 we compute the central charges and levels corresponding to the settings described above from a macroscopic viewpoint. When the transverse space is not given by $\mathrm{TN}_{m}$ or $\mathbb{R}^{4}$ the microscopic computation for these settings is still out of reach. We comment on this in the discussion of this chapter.

As in chapter 3 in sections 4.3 and 4.4 we only consider Calabi-Yau threefold fibrations in which the fiber degenerations do not lead to singularities of the whole fibration. The six-dimensional effective theories corresponding to these compactifications do not contain vector multiplets. In section 4.5 we study the inclusion of vector multiplets and in addition charged hypermultiplets which thus correspond to more general fibrations. In the $6 \mathrm{~d}$ bulk theory these vector multiplets furnish a gauge symmetry. From the point of view of the string propagating in $6 \mathrm{~d}$, this gauge symmetry acts as a flavour symmetry of the worldvolume theory living on the degrees of freedom of the string. These flavour symmetries have associated levels which we will compute from macroscopics. The inclusion of vector multiplets and charged hypermultiplets affects the computation of the central charges and levels corresponding to the symmetry of the transverse space. However as we will show, the expressions for those quantities as derived without the inclusion of vector multiplets will not change.

In section 4.2 we begin by outlining the various setups considered in this chapter, in particular reviewing ALE and ALF spaces. Section 4.3 is devoted to the computation of the classical part of the macroscopic central charges and levels of the $6 \mathrm{~d}$ strings considered here. The computation of the one-loop corrections to the charges and levels is the topic of section 4.4 In section 4.5 we then examine the inclusion of vectors and charged matter in the $6 \mathrm{~d}$ supergravity theory. We summarize our results in section 4.6 and comment on the microscopic computation of the computed quantities. 


\subsection{ALE/ALF transverse spaces}

In this section we shall give an overview of the various setups we consider in this chapter. All the cases considered here have the same underlying theory arising from compactifying F-theory on an elliptically fibered Calabi-Yau threefold. In the low energy limit this leads to an effective six-dimensional supergravity theory with chiral $\mathcal{N}=(1,0)$ supersymmetry. We consider black strings in this $6 \mathrm{~d}$ supergravity theory that arise from a D3-brane wrapped upon a curve $C \subset B$, where $B$ is the base of $\mathrm{CY}_{3}$. Additionally, these strings are wrapped upon a circle such that the strings are wrapped in an asymptotic $\mathbb{R} \times \mathrm{S}^{1} \times M$ geometry. In chapter 3 we considered strings that have a Taub-NUT space $M=\mathrm{TN}_{m}$ transversely. Instead in this chapter we replace $\mathrm{TN}_{m}$ with a more general Ricci-flat, hyper-Kähler, non-compact four-manifold. Such spaces have been classified into four categories depending on their asymptotic volume growth: ALE, ALF, ALG, ALH. At infinity the metrics approach a quotient of the flat metric on $\mathbb{R}^{4-k} \times \mathrm{T}^{k}$ where the fibration at infinity is trivial except in the $k=1$ case where it may be fibered. The metric is ALE when $k=0$, ALF when $k=1$, ALG when $k=2$ and finally ALH when $k=3$. We shall only consider two of these classes in this chapter: asymptotically locally Euclidean (ALE) and asymptotically locally flat (ALF) spaces ${ }^{1}$ For the benefit of the reader not familiar with these manifolds, or in need of a refresher, we summarize the salient points here.

A manifold is said to admit an ALE metric if it is diffeomorphic to $\mathbb{R}^{+} \times \mathrm{S}^{3} / \Gamma$, with $\Gamma$ a freely acting discrete subgroup of $S U(2)$, and has a metric that asymptotically approaches a quotient of the Euclidean flat space metric. The possible subgroups of $S U(2)$ admit an ADE-classification and are summarized in table 4.1. The metric is only explicitly known for $\Gamma=\mathbb{Z}_{m}$ in which case it is given by the Gibbons-Hawking metrid ${ }^{2}$ [107] which is a generalization of the Eguchi-Hanson metric [168. For the D- and E-series, the metric is known to exist [169 as a hyper-Kähler resolution of a quotient singularity $\mathbb{C}^{2} / \Gamma$, but its explicit form has yet to be established.

Likewise, ALF metrics are also diffeomorphic to $\mathbb{R}^{+} \times \mathrm{S}^{3} / \Gamma$. However, their metric instead asymptotically approaches the metric on $\left(\mathbb{R}^{3} \times S^{1}\right) / \Gamma$. In fact they are only known to exist for the A- and D-series and not for the E-series of the ADE-classification [170. As already mentioned in the introduction of this chapter, in the case of the A-series they are the Taub-NUT spaces with NUT charge $m[171$

\footnotetext{
${ }^{1}$ Note the ' $\mathrm{G}$ ' and ' $\mathrm{H}$ ' do not have a meaning like in the ' $\mathrm{E}$ ' and ' $\mathrm{F}$ ' cases but are named as such by induction.

${ }^{2}$ In section 2.4 .4 we considered a generalization of the metric in 107. Here we mean by GibbonsHawking the multi-centered metric in section 2.4.4 with $m_{\infty}=0$.
} 
constructed as a circle fibration over a base $\mathbb{R}^{3}$. At the location of the centers the circle fiber shrinks to zero size whereas at infinity the radius becomes constant. In the case of the D-series the metric is only known explicitly asymptotically but an implicit construction of the full metric may be found in 172 , see also 173 for the metric with quotient $\mathbb{D}_{1}$. Sufficiently far from the center, the metric can be approximated by a Taub-NUT space with $2 m$ centers, plus a contribution to the centers with a negative mass parameter, subject to a quotient by $\mathbb{Z}_{2}$ which acts by reflecting the coordinates on both $\mathbb{R}^{3}$ and $S^{1} 174$. In the black string solutions we always take the ALE and ALF spaces in their singular limit: i.e. with a $\mathbb{C}^{2} / \Gamma$ singularity at the center. The black string metric however, has a smooth near-horizon limit. Note that ALF spaces admit a circle at infinity whilst ALE spaces lack such a circle. This will play a crucial role in the calculation of one-loop corrections to the current levels in section 4.4

\begin{tabular}{lcc}
\hline$\Gamma \subset \mathrm{SU}(2)$ & $|\Gamma|$ & singularity type \\
\hline cyclic group $\mathbb{Z}_{m}$ & $m$ & $A_{m-1}$ \\
binary dihedral $\mathbb{D}_{m}^{*}$ & $4 m$ & $D_{m+2}$ \\
binary tetrahedral $\mathbb{T}^{*}$ & 24 & $E_{6}$ \\
binary octahedral $\mathbb{O}^{*}$ & 48 & $E_{7}$ \\
binary icosahedral $\mathbb{I}^{*}$ & 120 & $E_{8}$ \\
\hline
\end{tabular}

Table 4.1: The freely acting discrete subgroups of $S U(2)$.

It is known [131], that the near-horizon geometry of a black string with $\mathcal{N}=(0,4)$ supersymmetry must be

$$
\mathrm{AdS}_{3} \times \mathrm{S}^{3} / \Gamma
$$

For the A-series these black strings have a $U(1)_{L} \times S U(2)_{R}$ isometry group of the transverse space and may be spinning. For the D- and E-series the isometry group of the transverse space is $S U(2)_{R}$ and the strings are static. We may reinterpret these black string solutions as five-dimensional black holes by compactifying on the $\mathrm{S}^{1}$ wrapped by the D3 common to both setups. These $5 \mathrm{~d}$ black holes have a near-horizon geometry $\mathrm{AdS}_{2} \times \mathrm{S}^{3} / \Gamma$. For ALF spaces one may perform a further compactification on the asymptotic circle to obtain four-dimensional black holes. The entropy of these black holes follows from the left central charge and level (for the A-series) via Cardy's formula. 


\subsection{Macroscopic classical contributions}

In this section we compute the central charges and levels corresponding to the setups described in the preceding section using six-dimensional $\mathcal{N}=(1,0)$ supergravity which has been reviewed in section 3.3.1. The macroscopic configuration is that of a black string solution in $6 \mathrm{~d}$ with near-horizon geometry $\mathrm{AdS}_{3} \times \mathrm{S}^{3} / \Gamma$ which asymptotically approaches $\mathbb{R}^{1,1} \times M_{\Gamma \infty}$, with $M_{\Gamma \infty}$ denoting the asymptotic geometry of the ALE/ALF space $M_{\Gamma}$. In principle one could compute the central charges and levels by dimensionally reducing the six-dimensional action on the compact spherical part of the near-horizon geometry in order to obtain an effective action on $\mathrm{AdS}_{3} 55,58,136$. Using the AdS/CFT dictionary the central charges and levels then correspond to the coefficients of Chern-Simons terms in the dimensionally reduced action. Concretely, $k_{L, R}$ correspond (when the former is present) to the coefficients of the $U(1)_{L}$ (when present) and $S U(2)_{R}$ Chern-Simons terms respectively, whilst $c_{L}-c_{R}$ gives the coefficient of the gravitational Chern-Simons term.

However, it was shown in 60] (as we discussed in section 1.4.1) and the preceding chapter that this is not the full story and does not generically reproduce the correct microscopic results. Instead it was shown that one must perform the reduction on the spherical part at spatial infinity to take into account degrees of freedom living outside the horizon, known as hair, which contribute to the microscopic degeneracy 65, 66]. Clearly the near-horizon analysis by itself is insufficient as the distinction between the ALE and ALF cases is not visible there. As we have seen in chapter 3 in addition to performing the reduction at infinity one is required to include contributions from one-loop Chern-Simons terms arising from integrating out massive Kaluza-Klein (KK) modes.

We begin this section with generalizing the black string solution of section 3.3.2 to strings that have an arbitrary ALE or ALF space transversely. We then compute the classical corrections to the central charges and levels by reducing the six-dimensional action to three dimensions. We leave the computation of the one-loop contributions for the subsequent section.

\subsubsection{Black string solutions}

Here we consider supergravity theories without vector multiplets. Additionally we take all scalars in the hypermultiplets to be constant. Therefore, the relevant black strings are solutions of $\mathcal{N}=(1,0)$ supergravity coupled to a tensor multiplets. In section 3.3.2 we have constructed a black string solution 3.21 of this theory which has a Taub-NUT space transversely. As we have seen in section 2.4.2, one can 
generalize this metric to a solution of the form.

$$
\mathrm{d} \hat{s}_{6}^{2}=2 H^{-1}(\mathrm{~d} u+\beta)\left(\mathrm{d} v+\omega+\frac{1}{2} \mathcal{F}(\mathrm{d} u+\beta)\right)+H \mathrm{~d} s_{4}^{2}\left(M_{\Gamma}\right),
$$

where $\mathrm{d} s_{4}^{2}\left(M_{\Gamma}\right)$ is the ALE or ALF hyper-Kähler metric corresponding to the finite group $\Gamma$. The one-forms $\omega, \beta$ are defined on $M_{\Gamma}$ and, like the functions $H$ and $\mathcal{F}$, are independent of $u$ and $v$. They satisfy certain equations 2.82-2.85 that in principle can be solved once an explicit expression for the hyper-Kähler metric is given.

As in the solution (3.21) of the preceding chapter, $\partial_{u}$ is a Killing vector of the metric 4.2 which can be taken spacelike such that we find a metric for a black string wound in the $u$-direction. The near-horizon geometry of this string is $\mathrm{AdS}_{3} \times \mathrm{S}^{3} / \Gamma\left[131\right.$ with asymptotics given by 4.2 up to replacing $\mathrm{d} s_{4}^{2}\left(M_{\Gamma}\right)$ by its asymptotic metric $\mathrm{d} s_{4}^{2}\left(M_{\Gamma \infty}\right)$. The asymptotic space has a finite covering and its metric approaches a $\Gamma$-quotient of the following metric sufficiently fast:

$$
\mathrm{d} s_{4}^{2}\left(M_{\infty}\right)= \begin{cases}\mathrm{d} r^{2}+\frac{1}{4} r^{2}\left(\sigma_{1}^{2}+\sigma_{2}^{2}+\sigma_{3}^{2}\right) & M_{\Gamma} \text { is ALE }, \\ \mathrm{d} r^{2}+r^{2}\left(\sigma_{1}^{2}+\sigma_{2}^{2}\right)+\sigma_{3}^{2} & M_{\Gamma} \text { is ALF }\end{cases}
$$

Here $\sigma_{i}$ are the left-invariant forms on $\mathrm{S}^{3}$. We use the covering space to treat all quotients simultaneously. The charges of the black string follow from integrating the three-forms $\hat{G}_{\Gamma}^{\alpha}$ in the quotient space over the spherical part of the metric (given by the $\sigma_{i}$ part). As in 3.29 we choose the normalization

$$
\int_{M_{\Gamma}^{\mathrm{sph}}} \hat{G}_{\Gamma}^{\alpha}=\frac{1}{|\Gamma|} \int_{M^{\mathrm{sph}}} \hat{G}^{\alpha}=-(2 \pi)^{2} Q^{\alpha},
$$

by using the quotient map $\pi: M \rightarrow M_{\Gamma}, \hat{G}^{\alpha}=\pi^{*} \hat{G}_{\Gamma}^{\alpha}$. These charges are related to the microscopic charges $q^{\alpha}$ via

$$
Q^{\alpha}=q^{\alpha}-\frac{1}{4} p_{1}\left(M_{\Gamma}\right) c^{\alpha},
$$

where $p_{1}\left(M_{\Gamma}\right)$ is the first Pontryagin number of the space $M_{\Gamma}$. This identification may be proven in the same manner as in section 3.3 .2 . The shift in the charge is caused by the higher derivative term in the action. We can calculate the first Pontryagin numbers of the ALE and ALF spaces considered here using the index of the Dirac operator 175

$$
I_{1 / 2}=-\frac{1}{24} p_{1}\left(M_{\Gamma}\right)+\tilde{\epsilon}_{1 / 2}+\frac{1}{12} \frac{\epsilon}{|\Gamma|},
$$


where $\epsilon=0$ for ALE spaces and $\epsilon=1$ for ALF spaces. For all $\Gamma$ the index $I_{1 / 2}=0$ and furthermore $\tilde{\epsilon}_{1 / 2}$ takes values as given in table 4.2 175. The Pontryagin numbers can then be computed and this results in values which we also present in table 4.2 . Note that $2 m$ is indeed the value used for Taub-NUT in the preceding chapter.

\begin{tabular}{lccc}
\hline$\Gamma \subset \mathrm{SU}(2)$ & $\tilde{\epsilon}_{1 / 2}$ & $p_{1}\left(M_{\Gamma}\right)(\mathrm{ALE})$ & $p_{1}\left(M_{\Gamma}\right)(\mathrm{ALF})$ \\
\hline cyclic group $\mathbb{Z}_{m}$ & $\frac{m^{2}-1}{12 m}$ & $2 m-\frac{2}{m}$ & $2 m$ \\
binary dihedral $\mathbb{D}_{m}^{*}$ & $\frac{4 m^{2}+12 m-1}{48 m}$ & $2 m+6-\frac{1}{2 m}$ & $2 m+6$ \\
binary tetrahedral $\mathbb{T}^{*}$ & $\frac{167}{288}$ & $\frac{167}{12}$ & \\
binary octahedral $\mathbb{O}^{*}$ & $\frac{383}{576}$ & $\frac{383}{24}$ & \\
binary icosahedral $\mathbb{I}^{*}$ & $\frac{1079}{1440}$ & $\frac{1079}{60}$ & \\
\hline
\end{tabular}

Table 4.2: The value of $\tilde{\epsilon}_{1 / 2}$ and the first Pontryagin numbers for the different ALE and ALF spaces $M_{\Gamma}$.

\subsubsection{Classical contributions to central charges and levels}

In this section we determine the classical contributions to the central charges and levels generalizing the treatment in section 3.3 .3 for Taub-NUT as transverse space. Here 'classical' refers again to the contribution that is obtained by reducing the sixdimensional $\mathcal{N}=(1,0)$ pseudo-action to three dimensions along the compact space. The contributions to the central charges and levels are given by the coefficients of $3 \mathrm{~d}$ Chern-Simons terms. The reduction for the black strings with either ALE or ALF transverse space will be largely the same and therefore we treat them concurrently.

To perform the reduction one gauges the isometries of $S^{3} / \Gamma$ by introducing gauge fields for these symmetries. We can restrict ourselves to an ansatz for the covering space at asymptotic infinity which for the ALF spaces is given by taking $m=1$ in the ansatz of section 3.3.3.2. For the ALE covering space one additionally has to set $m_{\infty}=0$. In both cases we work in the limit that $r \rightarrow \infty$, even though this limit will only be explicitly taken in the end when necessary. The ansatz for the metric is thus given by

$$
\mathrm{d} \hat{s}_{6}^{2}=\mathrm{d} s_{\mathcal{M}_{3}}^{2}+\delta_{a b} e^{a} e^{b},
$$


where

$$
e^{a}= \begin{cases}\hat{e}^{a}-K_{L}^{a} A_{L}-K^{I a} A^{I} & \Gamma=\mathbb{Z}_{m}, \\ \hat{e}^{a}-K_{R}^{I a} A_{R}^{I} & \Gamma \neq \mathbb{Z}_{m} .\end{cases}
$$

The three-dimensional space $\mathcal{M}_{3}$ in 4.7 is the non-spherical part of the covering space. An explicit expression for the dreibein $\hat{e}^{a}$ in 4.8 is given by taking $m=1$ in 3.26 for $M_{\Gamma} \mathrm{ALF}$ and by additionally taking $m_{\infty}=0$ for $M_{\Gamma}$ ALE. Explicit expressions for the Killing vectors are given in (3.44).

We take the ansatz for the three-form flux $\hat{G}^{\alpha}$, the pull back of the three-form on the quotient space, to be $60,\left.136\right|^{3}$

$$
\hat{G}^{\alpha}=-Q^{\alpha}\left[(2 \pi)^{2}|\Gamma|\left(e_{3}-\chi_{3}\right)+\omega\left(\mathcal{M}_{3}\right)\right] .
$$

Here $\omega\left(\mathcal{M}_{3}\right)$ is a three-form on $\mathcal{M}_{3}$ whose form we do not specify. The three-form $e_{3}$ appearing in 4.9 is invariant under the isometries of the spacetime, integrates to unity over the spherical part

$$
\int_{M^{\mathrm{sph}}} e_{3}=1
$$

and has exterior derivative

$$
\mathrm{d} e_{3}= \begin{cases}\frac{1}{16 \pi^{2}} F_{L} \wedge F_{L}+\frac{1}{8 \pi^{2}} \operatorname{tr} F_{R} \wedge F_{R} & \Gamma=\mathbb{Z}_{m} \\ \frac{1}{8 \pi^{2}} \operatorname{tr} F_{R} \wedge F_{R} & \Gamma \neq \mathbb{Z}_{m} .\end{cases}
$$

An explicit form for this three-form can be obtained from (3.47) by setting $m=1$. For $\Gamma \neq \mathbb{Z}_{m}$ one additionally needs to set $A_{L}=0$. The three-form $\chi_{3}$ in 4.9 is given by

$$
\chi_{3}= \begin{cases}\frac{1}{16 \pi^{2}} A_{L} \wedge F_{L}+\frac{1}{8 \pi^{2}} \operatorname{tr}\left(A_{R} \wedge \mathrm{d} A_{R}+\frac{2}{3} A_{R}^{3}\right) & \Gamma=\mathbb{Z}_{m}, \\ \frac{1}{8 \pi^{2}} \operatorname{tr}\left(A_{R} \wedge \mathrm{d} A_{R}+\frac{2}{3} A_{R}^{3}\right) & \Gamma \neq \mathbb{Z}_{m}\end{cases}
$$

and is included to ensure that $\hat{G}^{\alpha}$ satisfies its Bianchi identity. Note that the pull back of 3.52 is equal to 4.9 as it should.

We are now ready to determine the classical contributions to the central charges and levels. In section 3.3 .3 we have seen that there are two terms in the supergravity

\footnotetext{
${ }^{3}$ Strictly the expression 4.9 only satisfies the self-duality constraint after setting the gauge fields to zero. However this is sufficient as the gauge fields are treated as fluctuations around the background.
} 
action contributing to $3 \mathrm{~d}$ Chern-Simons terms, namely

$$
\frac{1}{(2 \pi)^{3}} \int_{M_{6}}\left[-\frac{1}{4} g_{\alpha \beta} \hat{G}_{\Gamma}^{\alpha} \wedge \hat{*} \hat{G}_{\Gamma}^{\beta}-\frac{1}{8} \eta_{\alpha \beta} c^{\alpha} \hat{B}_{\Gamma}^{\beta} \wedge \operatorname{tr} \hat{\mathcal{R}} \wedge \hat{\mathcal{R}}\right] \subset S^{(6)} .
$$

We compute the contributions of both terms separately.

Two-derivative contribution. We compute the contribution of the two-derivative term by determining the gauge variation of the reduced action under either $U(1)_{L} \times$ $S U(2)_{R}$ gauge transformations for $\Gamma=\mathbb{Z}_{m}$ or $S U(2)_{R}$ gauge transformations for $\Gamma \neq \mathbb{Z}_{m}$. We parametrize the gauge transformations by $\Lambda$ and determine the variation of the three-dimensional action by reducing the variation of the six-dimensional action on $M_{\Gamma}^{\mathrm{sph}}$. By construction $e_{3}$ is invariant under all gauge transformations, consequently only the variation of $\chi_{3}$ contributes to the variation of the six-dimensional action. We find 4

$$
\begin{aligned}
\delta_{\Lambda} \mathcal{L}_{\mathrm{CS}}^{2-\text { der }} *_{3} 1 & =-\frac{1}{16 \pi^{3}} \int_{M_{\Gamma}^{\mathrm{sph}}} g_{\alpha \beta} \delta_{\Lambda} \hat{G}_{\Gamma}^{\alpha} \wedge \hat{*} \hat{G}_{\Gamma}^{\beta}=-\frac{1}{16 \pi^{3}|\Gamma|} \int_{M_{\mathrm{sph}}} g_{\alpha \beta} \delta_{\Lambda} \hat{G}^{\alpha} \wedge \hat{*} \hat{G}^{\beta} \\
& =\pi|\Gamma| \eta_{\alpha \beta} Q^{\alpha} Q^{\beta} \int_{M^{\mathrm{sph}}} \delta_{\Lambda} \chi_{3} \wedge e_{3}=\pi|\Gamma| \eta_{\alpha \beta} Q^{\alpha} Q^{\beta} \delta_{\Lambda} \chi_{3}
\end{aligned}
$$

where the third equality sign follows by using the (anti-)self-duality condition 3.20 . This gives the gauge variation of the three-dimensional action which from general considerations is given by

$$
\begin{aligned}
S_{\mathrm{CS}}^{2 \text {-der }} & =\pi|\Gamma| \eta_{\alpha \beta} Q^{\alpha} Q^{\beta} \int_{\mathcal{M}_{\Gamma 3}} \chi_{3} \\
& = \begin{cases}\frac{k_{L}^{2-\text { der }}}{8 \pi} \int_{\mathcal{M}_{\Gamma 3}} A_{L} \wedge F_{L}+\frac{k_{R}^{2-\text { der }}}{4 \pi} \int_{\mathcal{M}_{\Gamma 3}} \operatorname{tr}\left(A_{R} \wedge \mathrm{d} A_{R}+\frac{2}{3} A_{R}^{3}\right) & \Gamma=\mathbb{Z}_{m}, \\
\frac{k_{R}^{2-\text { der }}}{4 \pi} \int_{\mathcal{M}_{\Gamma 3}} \operatorname{tr}\left(A_{R} \wedge \mathrm{d} A_{R}+\frac{2}{3} A_{R}^{3}\right) & \Gamma \neq \mathbb{Z}_{m} .\end{cases}
\end{aligned}
$$

Comparing the two expressions we thus find for the central charges and levels

$$
\begin{aligned}
k_{L}^{2 \text {-der }} & =\frac{1}{2}|\Gamma| \eta_{\alpha \beta} Q^{\alpha} Q^{\beta} \quad \text { only for } \Gamma=\mathbb{Z}_{m}, \\
k_{R}^{2 \text {-der }} & =\frac{1}{2}|\Gamma| \eta_{\alpha \beta} Q^{\alpha} Q^{\beta}, \\
c_{L}^{2 \text {-der }} & =c_{R}^{2 \text {-der }},
\end{aligned}
$$

where $Q^{\alpha}$ is given by 4.5 . In writing 4.16 we have used that $c_{L}^{2 \text {-der }}-c_{R}^{2 \text {-der }}=0$ due to the absence of a $3 \mathrm{~d}$ gravitational Chern-Simons term after the reduction of the 2-derivative term in 4.13.

\footnotetext{
${ }^{4}$ In our conventions $\int_{M_{6}}=\int_{\mathcal{M}_{\Gamma 3}} \cdot \int_{M_{\Gamma}}^{\text {sph }}$, where $\mathcal{M}_{\Gamma 3}$ is the three-dimensional non-spherical part of the quotient space.
} 
Higher derivative contribution. We now determine the Chern-Simons terms that come from the reduction of the higher derivative term in 4.13 by integrating this term over the spherical part $M_{\Gamma}^{\text {sph }}$ :

$$
\mathcal{L}_{\mathrm{CS}}^{4-\mathrm{der}} *_{3} 1=\frac{1}{64 \pi^{3}} \int_{M_{\Gamma}^{\mathrm{sph}}} \eta_{\alpha \beta} c^{\alpha} \hat{G}_{\Gamma}^{\beta} \wedge \hat{\omega}_{\mathrm{grav}, \Gamma}^{\mathrm{CS}}=\frac{1}{64 \pi^{3}|\Gamma|} \int_{M^{\mathrm{sph}}} \eta_{\alpha \beta} c^{\alpha} \hat{G}^{\beta} \wedge \hat{\omega}_{\mathrm{grav}}^{\mathrm{CS}}
$$

We evaluate this integral by generalizing the expressions in appendix 3.A appropriately. This is possible because the results in that appendix are valid for Taub-NUT with arbitrary $m_{\infty}$ and here we work in the covering space of $M_{\Gamma}$ at asymptotic infinity. In particular 3.96 becomes

$$
\begin{aligned}
\int_{M^{\mathrm{sph}}} \hat{G}^{\alpha} \wedge \hat{\omega}_{\mathrm{grav}}^{\mathrm{CS}}=-Q^{\alpha}(2 \pi)^{2}|\Gamma| \int_{M^{\mathrm{sph}}}\left[e_{3} \wedge \omega_{\mathrm{grav}}^{\mathrm{CS}}\right. & +e_{3} \wedge \omega^{\mathrm{CS}}(X) \\
& \left.-\chi_{3} \wedge \omega^{\mathrm{CS}}\left(\hat{\omega}_{\mathrm{sph}}\right)\right]
\end{aligned}
$$

and the integrals 3.97 generalize to

$$
\begin{aligned}
\int_{M^{\mathrm{sph}}} e_{3} \wedge \omega_{\mathrm{grav}}^{\mathrm{CS}} & =-\omega_{\text {grav }}^{\mathrm{CS}}, \\
\int_{M^{\mathrm{sph}}} e_{3} \wedge \omega^{\mathrm{CS}}(X) & = \begin{cases}2 A_{L} \wedge F_{L} & \Gamma=\mathbb{Z}_{m} \\
0 & \Gamma \neq \mathbb{Z}_{m}\end{cases} \\
\int_{M^{\mathrm{sph}}} \chi_{3} \wedge \omega^{\mathrm{CS}}\left(\hat{\omega}_{\mathrm{sph}}\right) & =\frac{1+4 m_{\infty} r+10 m_{\infty}^{2} r^{2}+8 m_{\infty}^{3} r^{3}+2 m_{\infty}^{4} r^{4}}{\left(1+m_{\infty} r\right)^{4}} \times 16 \pi^{2} \chi_{3} .
\end{aligned}
$$

The result for ALE $M_{\Gamma}$ is then obtained by setting $m_{\infty}=0$ after which one has to take the limit $r \rightarrow \infty$. The latter limit does not change the result however which is given by

$$
\mathcal{L}_{\mathrm{CS}}^{4-\text { der }} *_{3} 1= \begin{cases}\frac{1}{16 \pi} \eta_{\alpha \beta} c^{\alpha} Q^{\beta}\left[\omega_{\text {grav }}^{\mathrm{CS}}-A_{L} \wedge F_{L}+2 \omega^{\mathrm{CS}}\left(A_{R}\right)\right] & \Gamma=\mathbb{Z}_{m}, \\ \frac{1}{16 \pi} \eta_{\alpha \beta} c^{\alpha} Q^{\beta}\left[\omega_{\mathrm{grav}}^{\mathrm{CS}}+2 \omega^{\mathrm{CS}}\left(A_{R}\right)\right] & \Gamma \neq \mathbb{Z}_{m} .\end{cases}
$$

Similarly, for ALF $M_{\Gamma}$ we just have to take the limit $r \rightarrow \infty$ in 4.19 such that we find

$$
\mathcal{L}_{\mathrm{CS}}^{4-\mathrm{der}} *_{3} 1=\frac{1}{16 \pi} \eta_{\alpha \beta} c^{\alpha} Q^{\beta}\left[\omega_{\mathrm{grav}}^{\mathrm{CS}}+4 \omega^{\mathrm{CS}}\left(A_{R}\right)\right]
$$


In particular note that this does not depend on $\Gamma$ which has to be contrasted with the ALE case. The corrections to the levels and central charges are then given by

$$
\begin{aligned}
& k_{L}^{4 \text {-der }}=-\frac{1}{2} \eta_{\alpha \beta} c^{\alpha} Q^{\beta} \quad \text { only for ALE } M_{\Gamma}, \quad \Gamma=\mathbb{Z}_{m}, \\
& k_{R}^{4 \text {-der }}= \begin{cases}\frac{1}{2} \eta_{\alpha \beta} c^{\alpha} Q^{\beta} & \operatorname{ALE} M_{\Gamma}, \\
\eta_{\alpha \beta} c^{\alpha} Q^{\beta} & \operatorname{ALF} M_{\Gamma},\end{cases} \\
& \left(c_{L}-c_{R}\right)^{4-\text { der }}=6 \eta_{\alpha \beta} c^{\alpha} Q^{\beta},
\end{aligned}
$$

where $Q^{\beta}$ is given by 4.5 . The last equality comes from comparing the $3 \mathrm{~d}$ gravitational Chern-Simons term to

$$
\mathcal{L}_{\mathrm{CS}} *_{3} 1 \supset \frac{c_{L}-c_{R}}{96 \pi} \omega_{\text {grav }}^{\mathrm{CS}} .
$$

We now add the results in 4.16 and 4.22 to find the classical contributions to the central charges and levels. This results in:

$$
\begin{aligned}
& k_{L}^{\text {class }}= \begin{cases}\frac{1}{2}|\Gamma| \eta_{\alpha \beta} Q^{\alpha} Q^{\beta}-\frac{1}{2} \eta_{\alpha \beta} c^{\alpha} Q^{\beta} & \operatorname{ALE~} M_{\Gamma}, \quad \text { only for } \Gamma=\mathbb{Z}_{m}, \\
\frac{1}{2}|\Gamma| \eta_{\alpha \beta} Q^{\alpha} Q^{\beta} & \operatorname{ALF~} M_{\Gamma}, \quad \text { only for } \Gamma=\mathbb{Z}_{m},\end{cases} \\
& k_{R}^{\text {class }}= \begin{cases}\frac{1}{2}|\Gamma| \eta_{\alpha \beta} Q^{\alpha} Q^{\beta}+\frac{1}{2} \eta_{\alpha \beta} c^{\alpha} Q^{\beta} & \operatorname{ALE} M_{\Gamma}, \\
\frac{1}{2}|\Gamma| \eta_{\alpha \beta} Q^{\alpha} Q^{\beta}+\eta_{\alpha \beta} c^{\alpha} Q^{\beta} & \operatorname{ALF} M_{\Gamma},\end{cases} \\
& \left(c_{L}-c_{R}\right)^{\text {class }}=6 \eta_{\alpha \beta} c^{\alpha} Q^{\beta} \text {, }
\end{aligned}
$$

where $Q^{\beta}$ is given by 4.5. The result for Taub-NUT is indeed equal to 3.63 and the result for $M_{\Gamma}=\mathbb{R}^{4}$, i.e. Gibbons-Hawking with $m=1$, is equal to the result in 76 .

When $M_{\Gamma}$ is ALE (4.24) is the full answer. On the other hand for ALF transverse space one must also include one-loop contributions originating from integrating out massive Kaluza-Klein modes. The content of the next section is devoted to the calculation of these one-loop contributions and showing that they only contribute in the ALF case and not the ALE one.

\subsection{Macroscopic quantum contributions for ALF transverse spaces}

In chapter 3 it was shown that including contributions from one-loop Chern-Simons terms is essential for reproducing the correct central charges and levels. These terms 
arise from integrating out massive Kaluza-Klein (KK) modes running in the loops of the relevant two-point functions. The relevant KK modes come from the chiral fields in six dimensions, i.e. the six-dimensional gravitinos, the spin- $\frac{1}{2}$ fermions in the tensor multiplets and the (anti-)self-dual two-forms. After dimensional reduction to three dimensions these fields lead to massive spin- $\frac{3}{2}$, spin- $\frac{1}{2}$ and chiral vector fields. The computation of the one-loop Chern-Simons terms is very similar to the one in section 3.4. Like we did there, we shall perform the calculation of the one-loop Chern-Simons terms in the $\mathrm{AdS}_{3} \times \mathrm{S}^{3} / \Gamma$ near-horizon geometry. We shall argue that for ALF transverse spaces the near-horizon computation matches the analogous computation at infinity. Further we will explain why for ALE transverse spaces the quantum contributions vanish when dimensionally reducing in the asymptotic geometry. As we have seen in section 3.4 the strategy to compute the quantum corrections is as follows. We first have to determine the relevant Kaluza-Klein spectrum after which we sum the contribution of each of the modes over this spectrum.

\subsubsection{Kaluza-Klein spectrum}

As in section 3.4.1 to determine the relevant KK spectrum we use that the $\mathcal{N}=(1,0)$ theory coupled to tensor multiplets can be obtained as a truncation of the $\mathcal{N}=(2,0)$ theory. The extra content in the $\mathcal{N}=(1,0)$ theory that we consider here are the hypermultiplets, but we assume that the modes associated to the fermions in these multiplets fall in the same representations as the fermions in the tensor multiplets. The relevant part of the KK spectrum for a reduction on $\mathrm{AdS}_{3} \times \mathrm{S}^{3}$ has been presented in section 3.4.1. For each mode the threedimensional Lorentz representation, the $\mathfrak{s o}(4)=\mathfrak{s u}(2)_{L} \oplus \mathfrak{s u}(2)_{R}$ representation following from the isometries of the three-sphere, and the sign of the mass are given. The six-dimensional fields obey symplectic-Majorana and reality conditions which are implemented by either only taking into account the modes that have $j_{L}^{3} \geq 0$ or $j_{R}^{3} \geq 0$, where $j_{L, R}^{3}$ are the eigenvalues of the generators $\mathfrak{u}(1)_{L, R} \subset \mathfrak{s u}(2)_{L, R}$. It then has to be determined which modes are projected out under the action of $\Gamma$, thereby determining the KK spectrum after the reduction on $\operatorname{AdS}_{3} \times \mathrm{S}^{3} / \Gamma$. In section 3.4 .1 we have seen that for $\Gamma=\mathbb{Z}_{m}$ invariant states satisfy $j_{L}^{3}=\frac{1}{2} m k, k \in \mathbb{Z}$, the symplectic-Majorana and reality conditions further refine this to $k \in \mathbb{Z}_{\geq 0}$. In appendix 3.B we found that for $\Gamma=\mathbb{D}_{m}^{*}$ the invariant representations have $j_{L}^{3}=m k$ with $k \in \mathbb{Z}_{\geq 0}$. The reality conditions imply in addition that we only keep states with $j_{R}^{3} \geq 0$. 
At asymptotic infinity the reduction is not performed on $S^{3} / \Gamma$ but on the compact part of the quotient of 4.3. It is therefore not a priori clear that the KK spectrum is the same as the one given above. For $\Gamma=\mathbb{Z}_{m}$ the reduction is on a squashed Lens space, where the radius of the two-sphere is taken to infinity. The Hopf circle still has finite radius and therefore the expectation is that the representation content of the KK spectrum will be the same as for $S^{3} / \mathbb{Z}_{m}$. We shall assume that this expectation is met and moreover that the signs of the masses do not change. For $\Gamma=\mathbb{D}_{m}^{*}$ there is still a two-sphere in the asymptotic geometry whose radius tends to infinity. However in contrast to the A-series there is no longer a circle, instead there is a segment with finite length, see for example [174]. Again the expectation is that we can use the previously determined KK spectrum.

This discussion sheds light on why we do not have to include one-loop ChernSimons terms for a transverse ALE space. From 4.3 it is easy to see that there is no compact space at asymptotic infinity on which one can perform a KK reduction, ergo there are no quantum corrections in the ALE case and the classical result of section 4.3 .2 is the final result.

\subsubsection{One-loop Chern-Simons terms from $\mathrm{KK}$ spectrum}

Having determined the spectrum, we may use the contribution of each mode to the three-dimensional $\mathfrak{u}(1)_{L}$ (only when $\left.\Gamma=\mathbb{Z}_{m}\right), \mathfrak{s u}(2)_{R}$ and gravitational Chern-Simons terms that can be found in table 3.1 and were determined using the Atiyah-Patodi-Singer index theorem. We need to sum the individual contributions over all modes. As we have seen in section 3.4 .2 it is necessary to regularize the summations using zeta-function regularization in order to obtain a finite result for the quantum corrections to the Chern-Simons terms. The relation between the coefficients $\alpha_{I}$ of these terms and the levels and central charges is given by (3.85). The quantum corrections for the A-series were computed in section 3.4 .2 and are given by

$$
\begin{aligned}
k_{L}^{\text {loop }} & =-\frac{m^{3}}{8} c_{1}(B)^{2}, \\
k_{R}^{\text {loop }} & =\frac{m^{3}}{24} c_{1}(B)^{2}+\frac{m}{3} c_{1}(B)^{2}+m, \\
\left(c_{L}-c_{R}\right)^{\text {loop }} & =6 m+2 m c_{1}(B)^{2},
\end{aligned}
$$

up to $\mathcal{O}(1)$ terms.

For the D-series there is no left-moving current isometry, hence there is no left level, $k_{L}$, to compute. As in the A-series case we sum the contributions of the 
individual representations over the KK spectrum of section 3.4.1 this time with $j_{L}^{3}=m k, k \in \mathbb{Z}_{\geq 0}$ and $j_{R}^{3} \geq 0$ instead of $j_{L}^{3}=\frac{1}{2} m k, k \in \mathbb{Z}_{\geq 0}$ as we had for the A-series. This is most easily implemented by first summing over all representations with $j_{L}^{3}=m k, k \in \mathbb{Z}_{\geq 0}$ and then imposing $j_{R}^{3} \geq 0$. Comparing with the condition in the A-series it is clear that the first step may be achieved by using the A-series results 4.25 and sending $m \rightarrow 2 m$. We must still impose the second condition. As the contribution for a given $j_{R}^{3}$ within a fixed $j_{R}$ representation is the same for $j_{R}^{3}$ and $-j_{R}^{3}$, imposing $j_{R}^{3} \geq 0$ can be performed effectively by dividing the result of the first step by two 5 Performing the sums for the D-series as we did in appendix 3.D for the A-series will result in the same linear order results. For the D-series, the one-loop contributions up to order $\mathcal{O}(1)$ in the charges are:

$$
k_{R}^{\text {loop }}=\frac{m^{3}}{6} c_{1}(B)^{2}+\frac{m}{3} c_{1}(B)^{2}+m, \quad\left(c_{L}-c_{R}\right)^{\text {loop }}=6 m+2 m c_{1}(B)^{2} .
$$

Adding the one-loop results to 4.24 gives the final results for the central charges and levels up to and including linear terms in the charges in case $M_{\Gamma}$ is ALF. We present the full expressions in section 4.6

\subsection{Non-abelian flavour symmetries}

We will now extend the preceding sections by including vector multiplets and charged hypermultiplets in the $6 \mathrm{~d} \mathcal{N}=(1,0)$ supergravity theory. These supergravity theories descend from compactifications of F-theory on elliptically fibered CalabiYau threefolds where the degeneration of the fiber along certain divisors results in singularities of the whole space. In the $6 \mathrm{~d}$ bulk theory, these vector multiplets furnish a gauge symmetry. From the point of view of the string propagating in $6 \mathrm{~d}$, which is microscopically realized by a D3-brane wrapping the curve $C$, this gauge symmetry in the bulk acts as a flavour symmetry of the worldvolume theory living on the degrees of freedom of the string. As before these flavour symmetries have associated levels which we will compute from macroscopics in this section. We refer to these levels as non-universal while in this section we refer to the levels corresponding to the symmetries of the transverse ALE/ALF space as universal. The inclusion of vector multiplets and charged hypermultiplets also affects the computation of the central charges and universal levels. However, as we will show

\footnotetext{
${ }^{5}$ We use the word effectively as the contribution from the $j_{R}^{3}=0$ terms is half of the actual result using this method. The difference, however, is $\mathcal{O}(1)$ in the charges and therefore for our purposes may be neglected.
} 
the results, expressed as in the preceding sections, will not change. We now first compute the levels corresponding to the flavour symmetries. As before the nonuniversal levels have a classical part, which we compute in subsection 4.5.1. and a quantum part which we compute in subsection 4.5.2. Subsequently we discuss in subsection 4.5 .3 how the central charges and universal levels change.

\subsubsection{Macroscopic classical contributions to non-universal levels}

In the following, we will assume for simplicity that the gauge group $G$ is a product of simple factors, i.e.

$$
G=\prod_{i} G_{i}
$$

where each simple factor $G_{i}$ descends from a 7 -brane stack in the underlying Ftheory model. Including vectors a new sector in the $6 \mathrm{~d}$ effective action must be included [37,

$$
\begin{aligned}
S^{(6)} \supset & \frac{-1}{(2 \pi)^{3}} \int_{M_{6}}\left[h_{U V} \mathcal{D} q^{U} \wedge \hat{*} \mathcal{D} q^{V}+\sum_{i} 2 \eta_{\alpha \beta} \hat{\jmath}^{\alpha} s_{i}^{\beta} \operatorname{tr} \hat{F}^{i} \wedge \hat{*} \hat{F}^{i}+\frac{1}{4} \eta_{\alpha \beta} \hat{B}_{\Gamma}^{\alpha} \wedge \hat{X}_{4}^{\beta}\right], \\
\hat{X}_{4}^{\alpha} & =\mathrm{d} \hat{X}_{3}^{\alpha}=\frac{1}{2} c^{\alpha} \operatorname{tr} \hat{\mathcal{R}} \wedge \hat{\mathcal{R}}-2 \sum_{i} s_{i}^{\alpha} \operatorname{tr} \hat{F}^{i} \wedge \hat{F}^{i}, \\
\hat{X}_{3}^{\alpha} & =\frac{1}{2} c^{\alpha} \hat{\omega}_{\text {grav }}^{\mathrm{CS}}-2 \sum_{i} s_{i}^{\alpha} \hat{\omega}^{\mathrm{CS}}\left(\hat{A}^{i}\right),
\end{aligned}
$$

where $S_{i}=s_{i}^{\alpha} \omega_{\alpha}$ are the Poincaré duals of the irreducible, effective divisors in $B$ on which the 7-brane stacks are localized. In other words, these are the codimension one loci in $B$, over which the elliptic fibration develops a singularity. The field strengths $\hat{F}^{i}$ thus correspond to the non-abelian symmetry factors $G_{i}$. Furthermore, the hypermultiplet scalars $q^{U}$ are now charged under these vectors and therefore are gauged using the gauge covariant derivative $\mathcal{D} q^{U}$.

\subsubsection{Black string solutions}

The relevant black string solutions are equal to the ones discussed in section 4.3.1. In particular we take the scalars in the hypermultiplets constant and take vanishing vectors. We now argue that also the identification between microscopic and macroscopic charges 4.5 does not change. Recall that the shift in this identification was due to the coupling $\hat{B}^{\alpha} \wedge \operatorname{tr} \hat{\mathcal{R}} \wedge \hat{\mathcal{R}}$ in the 6 d action. When the equation of motion of the two-form $\hat{B}^{\alpha}$ in the presence of a string-like source with 
(microscopic) charges $q^{\alpha}$ is then integrated over the space transverse to the string $M_{\Gamma}$, a non-trivial charge shift is found if

$$
p_{1}\left(M_{\Gamma}\right)=-\frac{1}{2} \frac{1}{(2 \pi)^{2}} \int_{M_{\Gamma}} \operatorname{tr} \hat{\mathcal{R}} \wedge \hat{\mathcal{R}} \neq 0 .
$$

In the presence of vector multiplets in $6 \mathrm{~d}$, we have a similar coupling, now involving $\hat{B}^{\alpha}$ and the gauge fields $\hat{F}^{i}$ in 4.28). This coupling is

$$
\int_{M_{6}} \sum_{i} \eta_{\alpha \beta} s_{i}^{\alpha} \hat{B}^{\beta} \wedge \operatorname{tr} \hat{F}^{i} \wedge \hat{F}^{i}
$$

and contributes to the equation of motion of $\hat{B}^{\alpha}$ similar to the $\operatorname{tr} \hat{\mathcal{R}} \wedge \hat{\mathcal{R}}$ coupling. These couplings would also contribute with independent shifts in the dictionary between $Q^{\alpha}$ and $q^{\alpha}$, provided that

$$
\int_{M_{\Gamma}} \operatorname{tr} \hat{F}^{i} \wedge \hat{F}^{i} \neq 0
$$

in the background. From an F-theory perspective, such an instanton can be seen as a gauge instanton on the 7-branes. This instanton configuration can induce D3-brane charge localized on the 7-brane dimensions transverse to the instanton configuration through a Chern-Simons coupling of the R-R four-form to the gauge fields ${ }^{6}$ However, we consider $6 \mathrm{~d}$ solutions without gauge instantons, such that 4.30 does not contribute to the dictionary 7 We therefore find that the original identification of micro- and macroscopic charges 4.5 remains valid.

\subsubsection{Classical contributions to non-universal levels}

Let us perform a dimensional reduction of the action 4.28 on the spherical part of the black string solution to determine the levels corresponding to the non-abelian symmetry factors $G_{i}$. We take the trivial reduction ansatz for the gauge fields, i.e.

$$
\hat{F}^{i}=F^{i}=\mathrm{d} A^{i}+A^{i} \wedge A^{i}
$$

where now $F^{i}$ has all its legs on the non-spherical part. To compute the classical part of the non-universal levels we need to determine the Chern-Simons terms corresponding to $F^{i}$ in the three-dimensional action. Looking at the action 4.28,

\footnotetext{
${ }^{6}$ This is similar to $\mathrm{D}(-1)$-brane charge induced on D3-branes via gauge instantons in $\mathcal{N}=4 \mathrm{SYM}$. In the case of the 7-branes, the coupling in question has the form $C_{4} \wedge \operatorname{tr} F \wedge F$.

${ }^{7}$ Such instanton configurations on Taub-NUT spaces were discussed in 176 178.
} 
we notice that the only potential source for Chern-Simons terms corresponding to $F^{i}$, are the terms

$$
\frac{1}{2(2 \pi)^{3}} \sum_{i} \eta_{\alpha \beta} s_{i}^{\alpha} \int_{M_{6}} \hat{B}_{\Gamma}^{\beta} \wedge \operatorname{tr} \hat{F}^{i} \wedge \hat{F}^{i} \subset S^{(6)}
$$

We compute

$$
\begin{aligned}
(2 \pi)^{3} \mathcal{L}_{\mathrm{CS}}^{\text {n.a. }} *_{3} 1 & =\frac{1}{2} \sum_{i} \eta_{\alpha \beta} s_{i}^{\alpha} \int_{M_{\Gamma}^{\mathrm{sph}}} \hat{B}_{\Gamma}^{\beta} \wedge \operatorname{tr} \hat{F}^{i} \wedge \hat{F}^{i} \\
& =\frac{1}{2|\Gamma|} \sum_{i} \eta_{\alpha \beta} s_{i}^{\alpha} \int_{M^{\mathrm{sph}}} \hat{\omega}^{\mathrm{CS}}\left(\hat{A}^{i}\right) \wedge \hat{G}^{\beta} \\
& =-(2 \pi)^{3} \frac{1}{4 \pi} \sum_{i} \eta_{\alpha \beta} s_{i}^{\alpha} Q^{\beta} \omega^{\mathrm{CS}}\left(A^{i}\right)=-(2 \pi)^{3} \sum_{i} \frac{k_{G_{i}}^{\text {class }}}{4 \pi} \omega^{\mathrm{CS}}\left(A^{i}\right),
\end{aligned}
$$

such that the classical part of the non-universal levels is equal to

$$
k_{G_{i}}^{\text {class }}=\eta_{\alpha \beta} s_{i}^{\alpha} Q^{\beta}
$$

As discussed in section 1.4.1 the sign of the Chern-Simons terms 4.34 implies that the corresponding Kac-Moody algebra in the 2d theory is left-moving.

\subsubsection{Macroscopic quantum contributions to non-universal levels for ALF transverse spaces}

As we have argued in section 4.4 for ALF transverse space we also have to include quantum contributions to the Chern-Simons terms in 3d. These are induced by chiral fields which couple to the gauge fields $\hat{A}^{i}$. These are given by the gauginos in the adjoint representations of $G_{i}$, and the hyperinos in hypermultiplets in representations

$$
\mathbf{R}=\bigotimes_{i} \mathbf{R}_{i}
$$

of $G$. Here $\mathbf{R}_{i}$ is the representation of the fermion under the gauge group factor $G_{i}$. To stay close to the notation of section 3.4 .1 we label the spin- $\frac{1}{2}$ field content with the notation

$$
\left(j_{L}, j_{R}, \mathbf{R}\right)^{\operatorname{sgn}(M)} .
$$


The spin- $\frac{1}{2}$ spectrum of the hypermultiplets and vector multiplets can then be listed as:

$$
\begin{aligned}
& 2 \bigoplus_{\mathbf{R}} x_{\mathbf{R}}\left[\bigoplus_{j_{L}=\frac{1}{2}}^{\infty}\left(j_{L}, j_{L} \pm \frac{1}{2}, \mathbf{R}\right)^{ \pm} \oplus\left(0, \frac{1}{2}, \mathbf{R}\right)^{+}\right] \\
& \oplus 2 \bigoplus_{i}\left[\bigoplus_{j_{L}=\frac{1}{2}}^{\infty}\left(j_{L}, j_{L} \pm \frac{1}{2}, \mathbf{a d}_{G_{i}} \otimes \mathbf{1}\right)^{\mp} \oplus\left(0, \frac{1}{2}, \mathbf{a d}_{G_{i}} \otimes \mathbf{1}\right)^{-}\right] .
\end{aligned}
$$

Here $x_{\mathbf{R}}$ denotes the multiplicity of the representation $\mathbf{R}$ in the spectrum of the $6 \mathrm{~d}$ supergravity theory and

$$
\operatorname{ad}_{G_{i}} \otimes 1 \equiv \mathbf{1} \otimes \cdots \otimes 1 \otimes \operatorname{ad}_{G_{i}} \otimes 1 \otimes \cdots \otimes 1
$$

denotes the representation, which is the adjoint of the gauge group factor $G_{i}$, but the singlet representation of all other gauge group factors $G_{j \neq i}$.

Along the lines of section 3.4 .2 we compute the contribution of a single massive spin- $\frac{1}{2}$ field, which transforms in the representation $\mathbf{R}=\bigotimes_{i} \mathbf{R}_{i}$ under $G$, to the Chern-Simons terms in $3 \mathrm{~d}$. The parity anomaly from such a massive field can be canceled by a counter term 158

$$
\pi \operatorname{sgn}(M) \int_{\mathcal{M}_{3}} Q_{\frac{1}{2}}(A, \omega)
$$

where

$$
\begin{aligned}
\mathrm{d} Q_{\frac{1}{2}}(A, \omega) & =\left.\hat{A}\left(\mathcal{M}_{3}\right) \wedge \operatorname{ch}(F)\right|_{4 \text {-form }}=\left.\hat{A}\left(\mathcal{M}_{3}\right) \wedge \bigwedge_{i} \operatorname{ch}\left(F^{i}\right)\right|_{4 \text {-form }}, \\
\hat{A}\left(\mathcal{M}_{3}\right) & =1+\frac{1}{(4 \pi)^{2}} \frac{1}{12} \operatorname{tr} \mathcal{R} \wedge \mathcal{R}+\ldots, \\
\operatorname{ch}\left(F^{i}\right) & =\operatorname{dim} \mathbf{R}_{i}+\frac{i}{2 \pi} \operatorname{tr}_{\mathbf{R}_{i}} F^{i}-\frac{1}{2} \frac{1}{(2 \pi)^{2}} \operatorname{tr}_{\mathbf{R}_{i}} F^{i} \wedge F^{i}+\ldots
\end{aligned}
$$

Note that $\operatorname{tr} F^{i}=0$, since we assumed that the factors $G_{i}$ are simple. We then find

$$
\begin{aligned}
\left.\mathrm{d} Q_{\frac{1}{2}}(A, \omega)\right|_{4 \text {-form }} & =\frac{\operatorname{dim} \mathbf{R}}{(4 \pi)^{2}} \frac{1}{12} \operatorname{tr} \mathcal{R} \wedge \mathcal{R}-\frac{1}{2} \frac{1}{(2 \pi)^{2}} \sum_{i} d_{i}(\mathbf{R}) \operatorname{tr}_{\mathbf{R}_{i}} F^{i} \wedge F^{i} \\
& =\frac{\operatorname{dim} \mathbf{R}}{(4 \pi)^{2}} \frac{1}{12} \operatorname{tr} \mathcal{R} \wedge \mathcal{R}-\frac{1}{2} \frac{1}{(2 \pi)^{2}} \sum_{i} d_{i}(\mathbf{R}) A_{\mathbf{R}_{i}} \lambda_{i} \operatorname{tr} F^{i} \wedge F^{i}
\end{aligned}
$$

where now

$$
\operatorname{dim} \mathbf{R}=\prod_{i} \operatorname{dim} \mathbf{R}_{i}, \quad d_{i}(\mathbf{R})=\prod_{j \neq i} \operatorname{dim} \mathbf{R}_{j}
$$


Here $\lambda_{i}$ are normalization constants of tr with respect to the trace over the fundamental representation of $G_{i}, \operatorname{tr}_{f}$, i.e. $\operatorname{tr}_{f}=\lambda_{i}$ tr. This normalization constant depends on $G_{i}$, and tr is used in the Chern-Simons terms in (4.34). Furthermore, the constants $A_{\mathbf{R}_{i}}$ are defined via the relation

$$
\operatorname{tr}_{\mathbf{R}_{i}} F^{i} \wedge F^{i}=A_{\mathbf{R}_{i}} \operatorname{tr}_{f} F^{i} \wedge F^{i}
$$

We therefore conclude that a single spin- $\frac{1}{2}$ fermion in the representation $\mathbf{R}$ of $G$ contributes with

$$
\alpha_{G_{i}}^{1 / 2}=-\frac{1}{8 \pi} \operatorname{sgn}(M) d_{i}(\mathbf{R}) A_{\mathbf{R}_{i}} \lambda_{i}
$$

to the Chern-Simons term of the gauge field $A^{i}$. Analogous to what we did in section 4.4 for the central charges and universal levels, we now sum this contribution over a projection of the Kaluza-Klein spectrum 4.38. We do this separately for $\operatorname{ALF} M_{\Gamma}$ with $\Gamma=\mathbb{Z}_{m}$ and $\Gamma=\mathbb{D}_{m}^{*}$.

One-loop corrections for $\boldsymbol{\Gamma}=\mathbb{Z}_{\boldsymbol{m}}$. Up to terms of $\mathcal{O}(1)$ the contribution from the hyperinos is given by

$$
\begin{aligned}
\text { (hyper) }=-2 \times \frac{\lambda_{i}}{8 \pi} \sum_{\mathbf{R}} x_{\mathbf{R}} d_{i}(\mathbf{R}) A_{\mathbf{R}_{i}} \sum_{k=1}^{\infty} \sum_{j_{L}=\frac{1}{2} m k}^{\infty}\left[\left(2\left(j_{L}+\frac{1}{2}\right)+1\right)\right. \\
=-\frac{\lambda_{i}}{2 \pi} \sum_{\mathbf{R}} x_{\mathbf{R}} d_{i}(\mathbf{R}) A_{\mathbf{R}_{i}} \sum_{k=1}^{\infty} \sum_{j_{L}=\frac{1}{2} m k}^{\infty} 1=-\frac{\lambda_{i}}{48 \pi} m \sum_{\mathbf{R}} x_{\mathbf{R}} d_{i}(\mathbf{R}) A_{\mathbf{R}_{i}} .
\end{aligned}
$$

We can now use

$$
\sum_{\mathbf{R}} x_{\mathbf{R}} d_{i}(\mathbf{R}) A_{\mathbf{R}_{i}}=\sum_{\mathbf{R}_{i}} x_{\mathbf{R}_{i}} A_{\mathbf{R}_{i}}
$$

to trade the sum over all product representations $\mathbf{R}$ of $G$ in favour of a sum over all representations $\mathbf{R}_{i}$ of the gauge group factor $G_{i}$. Here we defined $x_{\mathbf{R}_{i}}$ as the multiplicity of all hypermultiplet fermions, which transform in the representation $\mathbf{R}_{i}$ of $G_{i}$. We therefore find for the contribution from the hypermultiplet fermions

$$
\text { (hyper) }=-\frac{\lambda_{i}}{48 \pi} m \sum_{\mathbf{R}_{i}} x_{\mathbf{R}_{i}} A_{\mathbf{R}_{i}}+\mathcal{O}(1) \text {. }
$$


Likewise, we find for the contribution from the gauginos

$$
\begin{aligned}
\text { (gauge) } & =2 \times \frac{\lambda_{i}}{8 \pi} A_{\mathbf{a d}_{G_{i}}} \sum_{k=1}^{\infty} \sum_{j_{L}=\frac{1}{2} m k}^{\infty}\left[\left(2\left(j_{L}+\frac{1}{2}\right)+1\right)-\left(2\left(j_{L}-\frac{1}{2}\right)+1\right)\right] \\
& =\frac{\lambda_{i}}{48 \pi} m A_{\mathbf{a d}_{G_{i}}}
\end{aligned}
$$

again up to $\mathcal{O}(1)$ terms. The correction to the level $k_{G_{i}}$ is then

$$
k_{G_{i}}^{\text {loop }}=-4 \pi[(\text { hyper })+(\text { gauge })]=\frac{m}{12} \lambda_{i}\left(\sum_{\mathbf{R}_{i}} x_{\mathbf{R}_{i}} A_{\mathbf{R}_{i}}-A_{\mathbf{a d}_{G_{i}}}\right) .
$$

We now make use of the anomaly cancellation condition

$$
\lambda_{i}\left(\sum_{\mathbf{R}_{i}} x_{\mathbf{R}_{i}} A_{\mathbf{R}_{i}}-A_{\mathbf{a d}_{G_{i}}}\right)=6 \eta_{\alpha \beta} c^{\alpha} s_{i}^{\beta}=6 c_{1}(B) \cdot S_{i},
$$

and we find

$$
k_{G_{i}}^{\text {loop }}=\frac{m}{2} c_{1}(B) \cdot S_{i} .
$$

One-loop corrections for $\Gamma=\mathbb{D}_{m}^{*}$. In section 4.4 we have argued that it is sufficient (up to $\mathcal{O}(1)$ ) to take the result obtained for $\mathbb{Z}_{m}$, replace $m \rightarrow 2 m$ in the expression for the loop correction, and then divide by a factor of 2 . As the correction 4.52 is linear in $m$, we obtain with this procedure the same result for $\mathbb{D}_{m}^{*}$.

\subsubsection{Macroscopic quantum contributions to central charges and universal levels}

We now determine how vector multiplets and charged matter change the computation of one-loop corrections to the central charges and universal levels in section 4.4 It is straightforward to see, following the logic of section 3.4 .2 that a spin- $\frac{1}{2}$ state with $U(1)_{L}$ charge $j_{L}^{3}$, in the isospin $j_{R}$ representation of $\mathrm{SU}(2)_{R}$ and $\mathbf{R}$ of $G$ contributes with

$$
\begin{aligned}
\alpha_{L}^{1 / 2} & =\frac{1}{8 \pi} \operatorname{sgn}(M) \operatorname{dim} \mathbf{R}\left(j_{L}^{3}\right)^{2}\left(2 j_{R}+1\right), \\
\alpha_{R}^{1 / 2} & =-\frac{1}{12 \pi} \operatorname{sgn}(M) \operatorname{dim} \mathbf{R} j_{R}\left(j_{R}+1\right)\left(2 j_{R}+1\right), \\
\alpha_{\text {grav }}^{1 / 2} & =\frac{1}{192 \pi} \operatorname{sgn}(M) \operatorname{dim} \mathbf{R}\left(2 j_{R}+1\right),
\end{aligned}
$$


to respectively the $U(1)_{L}, S U(2)_{R}$ and gravitational Chern-Simons terms. These are just the values from table 3.1 multiplied by $\operatorname{dim} \mathbf{R}$. When summing over the spectrum we therefore get the same sums as in appendix 3.D except with all terms proportional to $n_{H}$ replaced by

$$
n_{H} \rightarrow \sum_{\mathbf{R}} x_{\mathbf{R}} \operatorname{dim} \mathbf{R}-\sum_{i} \operatorname{dim} \mathbf{a d}_{G_{i}}=n_{H}-n_{V},
$$

where $n_{H}$ and $n_{V}$ are respectively the number of hypermultiplets and the number of vector multiplets. In the derivation of the final expressions for the central charges and levels in the preceding sections, we used

$$
n_{H}=273-29 n_{T}, \quad n_{T}=9-c_{1}(B)^{2},
$$

to eliminate $n_{H}$ and $n_{T}$. The relations 4.55 are respectively the condition which ensures the cancellation of the gravitational anomaly in $6 \mathrm{~d}$ for the case $n_{V}=0$ and the number of tensor multiplets in terms of topological data on $B$. When $n_{V} \neq 0$ the conditions we use are

$$
n_{H}-n_{V}=273-29 n_{T}, \quad n_{T}=9-c_{1}(B)^{2} .
$$

Note that the condition imposed by cancellation of the gravitational anomaly in the case $n_{V} \neq 0$ is simply the one in 4.55 with $n_{H}$ replaced by $n_{H} \rightarrow n_{H}-n_{V}$. Since the only effect of including charged matter and vectors in the loop sums is (4.54), we find that the one-loop contributions to $c_{L}-c_{R}$ and $k_{L, R}$ (given in terms of $c_{1}(B)$ and $m$ ) computed in section 4.4 are still valid for $n_{V} \neq 0$ and with charged matter.

\subsection{Summary and discussion}

In this chapter we have used the macroscopic supergravity picture to compute the central charges and levels of $\mathcal{N}=(0,4)$ SCFTs corresponding to F-theory geometries $\mathbb{R} \times \mathrm{S}^{1} \times M_{\Gamma} \times \mathrm{CY}_{3}$ with a D3-brane wrapped around $\mathrm{S}^{1} \times C$. Here $M_{\Gamma}$ is either an ALE or an ALF space and $C \subset B$ is a curve in the base of $\mathrm{CY}_{3}$. The central charges and levels correspond to coefficients of Chern-Simons terms in the effective $3 \mathrm{~d}$ action after reducing the $6 \mathrm{~d}$ theory on the spherical part of the relevant black string solution. For ALE transverse spaces we have shown that the only contribution to these Chern-Simons terms is from the classical reduction of the action which we performed in section 4.3. From (4.5) and 4.24 we find that 
the results for the central charges and universal levels are

$$
\begin{aligned}
k_{L}=\frac{1}{2}|\Gamma| C^{2}- & \frac{1}{2}\left(\frac{1}{2}|\Gamma| p_{1}\left(M_{\Gamma}\right)+1\right) c_{1}(B) \cdot C \\
& +\frac{1}{8}\left(\frac{1}{4}|\Gamma| p_{1}\left(M_{\Gamma}\right)+1\right) p_{1}\left(M_{\Gamma}\right) c_{1}(B)^{2} \quad \text { only for } \Gamma=\mathbb{Z}_{m} \\
k_{R}=\frac{1}{2}|\Gamma| C^{2}- & \frac{1}{2}\left(\frac{1}{2}|\Gamma| p_{1}\left(M_{\Gamma}\right)-1\right) c_{1}(B) \cdot C \\
& +\frac{1}{8}\left(\frac{1}{4}|\Gamma| p_{1}\left(M_{\Gamma}\right)-1\right) p_{1}\left(M_{\Gamma}\right) c_{1}(B)^{2} \\
c_{L}= & c_{R}+6 c_{1}(B) \cdot C-\frac{3}{2} p_{1}\left(M_{\Gamma}\right) c_{1}(B)^{2}
\end{aligned}
$$

where $|\Gamma|$ may be read off of table 4.1 and $p_{1}\left(M_{\Gamma}\right)$ of table 4.2

In contrast, for ALF transverse spaces we must include, in addition to the classical contribution 4.24, the quantum corrections obtained in section 4.4 For the A-series this results in

$$
\begin{aligned}
k_{L} & =\frac{1}{2} m C^{2}-\frac{1}{2} m^{2} c_{1}(B) \cdot C, \\
k_{R} & =\frac{1}{2} m C^{2}-\frac{1}{2} m^{2} c_{1}(B) \cdot C+\frac{1}{6} m^{3} c_{1}(B)^{2}+c_{1}(B) \cdot C+m-\frac{1}{6} m c_{1}(B)^{2}, \\
c_{L}-c_{R} & =6 c_{1}(B) \cdot C+6 m-m c_{1}(B)^{2},
\end{aligned}
$$

as already presented in (3.87). For the D-series we find by combining 4.5, 4.24) and 4.26) that

$$
\begin{aligned}
& k_{R}=2 m C^{2}-\left(2 m^{2}+6 m-1\right) c_{1}(B) \cdot C+\frac{1}{6}\left(4 m^{3}+18 m^{2}+26 m-9\right) c_{1}(B)^{2}+m, \\
& c_{L}=c_{R}+6 c_{1}(B) \cdot C-(m+9) c_{1}(B)^{2}+6 m .
\end{aligned}
$$

All results presented here are up to linear terms in the charges, we do not include any $\mathcal{O}(1)$ terms.

We have also examined the inclusion of vector multiplets and charged matter in the $6 \mathrm{~d}$ supergravity theories. These vector multiplets correspond to gauge symmetries in the $6 \mathrm{~d}$ bulk theory and arise from singularities of the Calabi-Yau along divisors $S_{i}$. From the point of view of the string, these gauge symmetries act as non-abelian flavour symmetries of the worldvolume theory living on the degrees of freedom of the string. We have computed the levels associated to these symmetries from macroscopics. In case the transverse space is ALE, these levels are given by combining 4.5 and 4.35 , i.e.

$$
k_{G_{i}}=\left(C-\frac{1}{4} p_{1}\left(M_{\Gamma}\right) c_{1}(B)\right) \cdot S_{i} .
$$


When the transverse space is ALF we also have to add the one-loop corrections 4.52 such that the levels are given by

$$
k_{G_{i}}=C \cdot S_{i}
$$

up to $\mathcal{O}(1)$. These results are in agreement with results stated in the literature for $M_{\Gamma}=\mathbb{R}^{4} 179181$.

It would be very interesting to reproduce the central charges and levels computed in this chapter from a microscopic calculation. The only settings where this has been done (at least for the central charges and universal levels) is when the transverse space is either Taub-NUT [75] or flat space [76, 166]. As explained in chapter 3 for Taub-NUT one can employ the dual M-theory setting. When the transverse space is $\mathbb{R}^{4}$ one can work directly with the worldvolume theory of the D3-brane. To place it on the curve $C$ in the presence of a varying axio-dilaton one has to perform a topological duality twist 165, 166, 182. The central charges and universal levels then correspond to anomaly coefficients in the resulting $2 \mathrm{~d}$ theory. Generalizing the latter computation to arbitrary ALE $M_{\Gamma}$ is problematic. The worldvolume theory of a D3-brane probing $M_{\Gamma}$ is known [183, 184], however the topological duality twist is not understood yet in a way it could be applied to this theory. Therefore, currently we cannot place it on the curve $C$. In case the transverse space is ALF corresponding to the D-series it is completely unclear what to do. However, if we do not include D7-branes one only needs a topological twist at the microscopic side which is well-understood. Therefore, the topic of chapter 5 will be to study black strings arising from a D3-brane wrapped on a curve $C \subset \mathrm{K} 3$ with asymptotic geometry $\mathbb{R} \times M_{\Gamma} \times \mathrm{S}^{1} \times \mathrm{K} 3$. As we did in this chapter, the macroscopic computations can be done for arbitrary ALE or ALF $M_{\Gamma}$. In case of an ALE transverse space the microscopic computation can also be done using the worldvolume theory of the D3-brane and employing a topological twist. When the transverse space is $\mathrm{TN}_{m}$ we can again employ the dual M-theory setting. 



\section{Black strings in type IIB with ALE/ALF spaces transversely}

In the preceding two chapters we have studied black strings emerging in F-theory compactifications. In particular we have studied D3-branes in an asymptotic $\mathbb{R} \times M_{\Gamma} \times \mathrm{S}^{1} \times \mathrm{CY}_{3}$ geometry where the D3-branes are wrapped on $\mathrm{S}^{1} \times C, C \subset B$ being a curve in the base of the elliptically fibered $\mathrm{CY}_{3}$. The space $M_{\Gamma}$ transverse to the black string is either asymptotically locally Euclidean (ALE) or asymptotically locally flat (ALF). We have computed central charges and levels of the $\mathcal{N}=(0,4)$ superconformal field theories (SCFTs) dual to these settings from macroscopic considerations. However, as we discussed in section 4.6 computing this data using a microscopic description is more difficult. Only when the transverse space is given by either Taub-NUT or by $\mathbb{R}^{4}$ it is known how to do this. In order to study black strings with an ALE or ALF transverse space both using a macroscopic and a complementary microscopic description we take a step back in this chapter, which is based on [3], and we study type IIB settings without 7-branes. More specifically, we consider black strings in compactifications of type IIB string theory on K3.

\subsection{Introduction}

As in the preceding two chapters the black strings arise from D3-branes, now wrapped in an asymptotic geometry $\mathbb{R} \times \mathrm{S}^{1} \times M_{\Gamma} \times \mathrm{K} 3$. The D3-branes are wrapped on $\mathrm{S}^{1} \times C$ with $C \subset \mathrm{K} 3$ a curve in K3. The transverse space $M_{\Gamma}$ is again either ALE or ALF and is defined by a choice of discrete subgroup $\Gamma \subset S U(2)$ as reviewed in section 4.2 Some of the black strings considered in this chapter have not been considered before in the literature. In particular, black strings arising from D3branes probing $M_{\Gamma}$ corresponding to the D- and E-series in the ADE-classification of subgroups of $S U(2)$ have not been studied previously. As in the preceding chapter, microscopically the strings are dual to $(0,4)$ SCFTs which admit both left- and right-moving central charges $c_{L, R}$. They also always admit a right-moving $S U(2)_{R}$ current algebra and for the A-series in addition a left-moving $U(1)_{L}$ current algebra, each with an associated level. In all cases the $S U(2)_{R}$ is identified with the $S U(2)_{r}$ 
R-symmetry of the small superconformal algebra of a $2 \mathrm{~d} \mathcal{N}=(0,4)$ SCFT and supersymmetry implies that the right-moving central charge and right-moving level are related via $c_{R}=6 k_{R} 1^{1}$ In this chapter we compute the central charges and levels corresponding to the settings described above both from a macroscopic and microscopic viewpoint.

Macroscopically we shall work exclusively in six-dimensional $\mathcal{N}=(2,0)$ supergravity 109,185 , which is obtained by compactifying type IIB on K3 [186]. For computing the various central charges and current algebra levels corresponding to the black string solutions of this theory we use the techniques introduced in section 1.4.1 and chapter 3 These macroscopic calculations are carried out in section 5.2

Alternatively, we could have chosen to look at these solutions directly in type IIB supergravity and compute the central charges there. The geometries of wrapped D3-brane solutions giving rise to $\mathrm{AdS}_{3}$ near-horizons were classified in 187]. This was further extended in 131,132 to include seven-branes in addition to the D3branes. The analysis performed in [131] shows that for wrapped D3-brane solutions preserving $\mathcal{N}=(0,4)$ supersymmetry the geometry is essentially unique and takes the form $\mathrm{AdS}_{3} \times \mathrm{S}^{3} / \Gamma \times \mathrm{CY}_{2}$. The central charges for certain $2 \mathrm{~d}$ CFTs were computed in 131] and one could in principle perform similar computations here, however we will work exclusively in six dimensions.

Microscopically the central charges and levels follow from the construction of new $2 \mathrm{~d} \mathcal{N}=(0,4)$ SCFTs. When $M_{\Gamma}$ is ALE these arise from wrapping known $4 \mathrm{~d} \mathcal{N}=2$ quiver gauge theories on a Riemann surface. Wrapping $4 \mathrm{~d} \mathcal{N}=2$ quiver theories on Riemann surfaces is in itself not new, see for example [188. However, in the context of black hole microstate counting this is the first instance of such a $2 \mathrm{~d}$ construction. The 4d parent quiver gauge theories are given by projections of non-abelian $\mathcal{N}=4$ supersymmetric Yang-Mills preserving $\mathcal{N}=2$ supersymmetry [183, 184. One then wishes to place the theory on a curve $C$ inside K3. In order to preserve $\mathcal{N}=(0,4)$ in $2 \mathrm{~d}$ this requires a particular topological twist to be performed. We then compute the central charges and levels and find a perfect matching to the macroscopic computations. When $M_{\Gamma}$ is ALF and $\Gamma=\mathbb{Z}_{m}$ corresponding to the A-series, we can compute the central charges and levels by considering a dual M-theory setting. In this case $M_{\Gamma}$ is the Taub-NUT space and we can T-dualize along the NUT-circle to obtain a type IIA setting. Lifting this to M-theory results in M-theory on

\footnotetext{
${ }^{1}$ This is a subtle point and as we discussed in section 1.4 .1 technically only applies when one decouples the contributions of the center of mass modes, see 60]. However as we only compute the levels and $c_{L}-c_{R}$ on both sides this will not matter for the matching between the microscopics and the macroscopics. We discuss this issue in more detail in section 5.3 .1
} 
$\mathrm{K} 3 \times \mathrm{T}^{2}$ with an M5-brane that wraps $C \times \mathrm{T}^{2}$ and $m$ M5-branes wrapping K3. These M5-branes combine into a single M5-brane wrapping $C \times \mathrm{T}^{2}+m \mathrm{~K} 3$ when the corresponding class is very ample. From this duality frame it is possible to determine the central charges $45,60,189]$. For the D-series such a clean description is not possible, we shall comment on this case further later.

In section 5.2 we begin with the computation of the macroscopic central charges and levels of the $6 \mathrm{~d}$ strings considered here. Beginning with a discussion on sixdimensional $\mathcal{N}=(2,0)$ supergravity and its relevant black string solutions, section 5.2 proceeds with the calculation of the classical and quantum contributions to the central charges and levels before a summary section collating the macroscopic results. The complementary microscopic calculation of the central charges and levels, split between ALE and ALF spaces, is performed in section 5.3. We find perfect agreement with the macroscopic results of section 5.2 . We relegate some technical material to the appendices.

\subsection{Macroscopics}

In this section we compute the central charges and levels corresponding to black strings arising from a D3-brane wrapped in an asymptotic geometry $\mathbb{R} \times M_{\Gamma} \times \mathrm{S}^{1} \times \mathrm{K} 3$ where $M_{\Gamma}$ is an ALE or ALF space, using six-dimensional $\mathcal{N}=(2,0)$ supergravity 109, 185, 186. The brane wraps $\mathrm{S}^{1} \times C$ with $C \subset \mathrm{K} 3$ a curve. As we shall review below, compactifying type IIB supergravity on $\mathrm{K} 3$ results in a gravity multiplet coupled to 21 tensor multiplets 109 186. In this section we will be very brief since the computations follow the ones in the preceding chapter closely. We reduce the $6 \mathrm{~d}$ supergravity theory on the spherical part of the relevant black string solution to obtain an action in 3d. The central charges and levels correspond to coefficients of Chern-Simons terms in 3d. As before, we perform this reduction at asymptotic infinity and we have to additionally compute one-loop contributions to the Chern-Simons terms in case of ALF transverse spaces. The main difference with the preceding chapters is that we work with $\mathcal{N}=(2,0)$ supergravity instead of $\mathcal{N}=(1,0)$ supergravity. The higher derivative term that was very important in the preceding two chapters is absent in this case such that the only 'classical' contribution to the central charges and levels comes from the two-derivative term in the action. Additionally we do not have the shift in the charge caused by this higher derivative term.

We begin this section with a review of six-dimensional $\mathcal{N}=(2,0)$ supergravity arising from type IIB supergravity compactified on K3. We then compute the 
classical and quantum corrections to the central charges and levels by first reducing the six-dimensional action to three dimensions and then including the one-loop contributions. We conclude the section with a summary of the results obtained therein in anticipation of comparing with the microscopic computations in the subsequent section.

\subsubsection{Six-dimensional supergravity from type IIB on $\mathrm{K} 3$}

The massless bosonic field content of type IIB supergravity includes a metric, a complex scalar, two real two-forms and a four-form with self-dual field strength. The cohomology of the manifold K3 (see section 1.2.3.2) admits one scalar, three self-dual two-forms, nineteen anti-self-dual two-forms and one four-form. As usual the reduction to the massless $6 \mathrm{~d}$ sector follows by expanding the various type IIB supergravity fields in terms of the generators of the cohomology of K3. Reducing the four-form leads to one scalar, three self-dual rank two tensors and nineteen anti-self-dual rank two tensors. The two 10d two-forms each lead to twenty-two scalars, one self-dual rank two tensor and one anti-self-dual rank two tensor in 6d. The complex scalar trivially reduces to two real scalars, whilst the metric leads to the graviton in $6 \mathrm{~d}$ and fifty-eight scalars. In total there are 105 scalars, 21 anti-self-dual rank two tensors, five self-dual rank two tensors and the graviton.

Having determined the massless bosonic fields in $6 \mathrm{~d}$ we may now rearrange them into $6 \mathrm{~d} \mathcal{N}=(2,0)$ multiplets. The various representations, labeled by the spins $\left(j_{1}, j_{2}\right)$ of the little group $S O(4) \simeq S U(2)_{1} \times S U(2)_{2}$, are:

- one gravity multiplet: $(1,1) \oplus 4\left(1, \frac{1}{2}\right) \oplus 5(1,0)$, containing one graviton, 2 left-handed gravitinos and five self-dual rank two tensors,

- $n_{T}$ tensor multiplets: $(0,1) \oplus 4\left(0, \frac{1}{2}\right) \oplus 5(0,0)$, containing one anti-self-dual rank two tensor, two right-handed tensorinos and 5 real scalars.

As sketched above, the compactification on K3 results in a theory coupled to $n_{T}=21$ tensor fields. This is the exact number of tensors necessary for the theory to be anomaly free and is in fact the unique anomaly-free six-dimensional $\mathcal{N}=(2,0)$ theory 186 . Uniqueness follows from the fact that the only possible matter multiplet in chiral $\mathcal{N}=(2,0)$ supergravity is the tensor multiplet and that the cancellation of gravitational anomalies requires $n_{T}=21$.

To perform computations it is advantageous to collectively denote the tensors in the gravity- and tensor multiplets by $\hat{B}^{\alpha}$, with $\alpha=1, \ldots, 26$, and their corresponding 
(self-dual) field strengths as $\hat{G}^{\alpha}=\left.\mathrm{d} \hat{B}^{\alpha}\right|^{2}$ The 105 scalars in the tensor multiplets parametrize the coset

$$
\mathcal{M}_{\text {tensor }}=\frac{S O(5,21)}{S O(5) \times S O(21)} \text {. }
$$

It turns out that in order to parametrize the scalar manifold it is more convenient to use the 130 scalars $\hat{\jmath}_{k}^{\alpha}, k=1, \ldots, 5$, satisfying the 25 constraints

$$
\Omega_{\alpha \beta} \hat{\jmath}_{k}^{\alpha} \hat{\jmath}_{l}^{\beta}=\delta_{k l}
$$

Here $\left(\Omega_{\alpha \beta}\right)$ is the $S O(5,21)$ invariant constant metric with mostly minus signature. It may be used to define a positive definite metric via

$$
g_{\alpha \beta}=2 \hat{\jmath}_{k \alpha} \hat{\jmath}_{\beta}^{k}-\Omega_{\alpha \beta}, \quad \hat{\jmath}_{k \alpha} \equiv \Omega_{\alpha \beta} \hat{\jmath}_{k}^{\beta}, \quad \hat{\jmath}^{k \alpha} \equiv \delta^{k l} \hat{\jmath}_{l}^{\alpha} .
$$

With this formalism the bosonic part of the pseudo-action is given by 185$]^{3}$

$$
S^{(6)}=\frac{1}{(2 \pi)^{3}} \int_{M_{6}}\left[\frac{1}{2} \hat{R} \hat{*} 1-\frac{1}{4} g_{\alpha \beta} \hat{G}^{\alpha} \wedge \hat{*} \hat{G}^{\beta}-\frac{1}{2}\left(\hat{\jmath}_{k \alpha} \hat{\jmath}_{\beta}^{k}-\Omega_{\alpha \beta}\right) \mathrm{d} \hat{\jmath}_{l}^{\alpha} \wedge \hat{*} \mathrm{~d} \hat{\jmath} l \beta\right],
$$

where we chose conventions such that $\kappa_{6}^{2}=(2 \pi)^{3}$. This is a pseudo-action since the self-duality constraints for the tensors,

$$
g_{\alpha \beta} \hat{*} \hat{G}^{\beta}=\Omega_{\alpha \beta} \hat{G}^{\beta},
$$

do not follow from the action and must be imposed by hand at the level of the equations of motion.

\subsubsection{Black string solutions}

Recall that we are interested in black strings arising from wrapping a D3-brane on a curve inside K3. As such we cannot turn on tensor fields arising from the reduction of the two 10d two-forms, each providing a self-dual and anti-self-dual tensor field. Moreover, preservation of supersymmetry implies that the Poincaré dual of the wrapped curve is in $H^{1,1}(K 3) 131,190,191$. This implies that the six-dimensional string is only charged under tensors arising from the reduction of the four-form

\footnotetext{
${ }^{2}$ We use the same notation as we did in the preceding two chapters for the $\mathcal{N}=(1,0)$ theory. This is convenient because as we will show in section 5.2 .2 the relevant black string solutions are given by a subset of the $\mathcal{N}=(1,0)$ solutions of section 4.3 .1 embedded into the $\mathcal{N}=(2,0)$ theory.

${ }^{3}$ Note that our conventions differ with respect to 185 , i.e. $\hat{G}_{\text {there }}^{\alpha}=\frac{1}{2} \hat{G}_{\text {here }}^{\alpha}$.
} 
along two-forms in $H^{1,1}(K 3)$. Consequently we may only have non-vanishing values for one self-dual tensor and 19 anti-self-dual tensors. Note that these are exactly the tensors we find in $\mathcal{N}=(1,0)$ supergravity coupled to 19 tensor multiplets. As we discussed in section 4.3.1 this theory has black string solutions with a transverse ALE or ALF space. These solutions may be embedded into the $\mathcal{N}=(2,0)$ theory by setting the scalars $\hat{\jmath}_{k}^{\alpha}=\delta_{k}^{27-\alpha}$ for $k=2,3,4,5$ and $\hat{\jmath}_{1}^{\alpha}=0$ for $\alpha=21, \ldots, 26{ }^{4}$ Therefore, from now on, $\alpha=1, \ldots, 20$, and the scalars $\hat{\jmath}^{\alpha} \equiv \hat{\jmath}_{1}^{\alpha}$ satisfy

$$
\Omega_{\alpha \beta} \hat{\jmath}^{\alpha} \hat{\jmath}^{\beta}=1
$$

where $\left(\Omega_{\alpha \beta}\right)$ is the (canonical) $S O(1,19)$ invariant constant metric with mostly minus signature. It can be identified with the metric on $H^{1,1}(\mathrm{~K} 3)$

$$
\Omega_{\alpha \beta}=\eta_{\alpha \beta}=\int_{\mathrm{K} 3} \omega_{\alpha} \wedge \omega_{\beta} .
$$

The black string solutions have been discussed in section 4.3.1 In particular, their metric is given by 4.2 and their charges are defined as in (4.4). These charges are related to the microscopic charges $q^{\alpha}$ via

$$
Q^{\alpha}=q^{\alpha}
$$

This identification may be proven in a similar manner as in section 3.3 .2 and has to be contrasted with the identification (4.5) of the previous chapter. Note that here we do not find a shift in the charge. This is because the action in the K3 case does not include a higher derivative term $\propto \int_{M_{6}} \hat{B}^{\alpha} \wedge \operatorname{tr} \hat{\mathcal{R}} \wedge \hat{\mathcal{R}}$ present in the F-theory case which leads to such a shift.

\subsubsection{Classical contributions to central charges and levels}

The goal of this section is to determine the classical contribution to the central charges and levels, i.e. the contribution coming from the reduction of the sixdimensional $\mathcal{N}=(2,0)$ pseudo-action to three dimensions along the compact space. Since this contribution comes from the $3 \mathrm{~d}$ Chern-Simons terms, we only need to consider the part of the pseudo-action (5.4) that can give rise to terms of this form, specifically the term:

$$
-\frac{1}{32 \pi^{3}} \int_{M_{6}} g_{\alpha \beta} \hat{G}^{\alpha} \wedge \hat{*} \hat{G}^{\beta} \subset S^{(6)} .
$$

\footnotetext{
${ }^{4}$ For later simplicity we have taken a non-canonical choice for $\Omega$. We take the first diagonal entry to be + , the next 21 - and the last four entries to be + .
} 
Note that this term is the same as the two-derivative term in 4.13 and that in contrast to that action we do not have a higher derivative term. Since we already showed that modulo the identification with the microscopic charges the black string solutions relevant in this chapter are a subset of the ones in section 4.3.1. we can use the results in section 4.3.2 In particular the ansätze for the metric and three-form are 4.7 and 4.9 respectively. Following the steps in section 4.3 .2 we find that the classical contribution is equal to 4.16 which with the charge identification 5.8 becomes

$$
\begin{aligned}
k_{L}^{\text {class }} & =\frac{1}{2}|\Gamma| \eta_{\alpha \beta} q^{\alpha} q^{\beta}=\frac{1}{2}|\Gamma| C \cdot C \quad \text { only for } \Gamma=\mathbb{Z}_{m}, \\
k_{R}^{\text {class }} & =\frac{1}{2}|\Gamma| C \cdot C \\
c_{L}^{\text {class }} & =c_{R}^{\text {class }}
\end{aligned}
$$

where $|\Gamma|$ may be read off of table 4.1 and we have introduced the notation

$$
C \cdot C=\int_{\mathrm{K} 3} C \wedge C=\eta_{\alpha \beta} q^{\alpha} q^{\beta}
$$

It is tempting to set $c_{R}=6 k_{R}$ using the field theory result, however as we discussed in section 1.4.1 and will explicitly show in section 5.3.1 this is only true modulo center of mass modes.

We note that if we had performed the reduction in the near-horizon geometry we would have obtained exactly the same results as in 5.10. This is a quirk of the current setup, as we have showed in chapter 3 in general a difference in the two reductions is possible when the supergravity action contains a higher derivative term, but as such a term is missing here the results agree.

As we argued in chapter 4 (5.10) gives the full answer when $M_{\Gamma}$ is ALE. For ALF transverse spaces one must also include one-loop contributions originating from integrating out massive Kaluza-Klein modes. We compute these contributions in the subsequent section.

\subsubsection{Quantum contributions for ALF transverse spaces}

We now compute the contribution to the central charges and levels from one-loop Chern-Simons terms that arise from integrating out massive chiral Kaluza-Klein modes which run in the loops of the relevant two-point functions. We do this computation in the near-horizon geometry but as explained in section 4.4 for ALF transverse spaces this gives the same result as when performed at asymptotic infinity. 
For ALE spaces this is not the case and there are no quantum contributions at asymptotic infinity which is why 5.10 is the full answer. The computation for the setting in this chapter is very similar to the ones in the previous two chapters. That is, we determine the relevant Kaluza-Klein modes and sum the contribution of each of the modes over this spectrum.

The relevant Kaluza-Klein spectrum is however different from the previous two chapters because we have a $6 \mathrm{~d} \mathcal{N}=(2,0)$ theory instead of a $\mathcal{N}=(1,0)$ theory. The relevant KK modes come from the 2 six-dimensional gravitinos, $2 n_{T}=42$ tensorinos, 5 self-dual rank two tensors and $n_{T}=21$ anti-self-dual rank two tensors. The gravitinos and tensorinos are given by two Weyl fermions obeying a symplecticMajorana condition and the tensors are subject to a reality condition. The gravitinos give rise to three-dimensional spin- $\frac{3}{2}$ and spin- $\frac{1}{2}$ particles, the tensorinos to spin- $\frac{1}{2}$ particles and the (anti-)self-dual tensors to chiral vector fields. The KK spectrum of $\mathrm{AdS}_{3} \times \mathrm{S}^{3}$ was computed in 152,153 . Initially we will not take into account the symplectic-Majorana nor reality condition, instead imposing these later. As in section 3.4.1 the representations are labeled in terms of $\mathfrak{s u}(2)_{L} \oplus \mathfrak{s u}(2)_{R}$ and the sign of the mass, $\operatorname{sgn}(M)$, as $\left(j_{L}, j_{R}\right)^{\operatorname{sgn}(M)}$. The representations are:

- Spin- $\frac{3}{2}$ :

$$
4 \bigoplus_{j_{L}=\frac{1}{2}}^{\infty}\left(j_{L}, j_{L} \pm \frac{1}{2}\right)^{\mp}
$$

- Spin- $\frac{1}{2}$ :

$$
\begin{gathered}
4 \bigoplus_{j_{L}=\frac{3}{2}}^{\infty}\left(j_{L}, j_{L} \pm \frac{3}{2}\right)^{\mp} \oplus 4 \bigoplus_{j_{L}=0}^{1}\left(j_{L}, j_{L}+\frac{3}{2}\right)^{-} \oplus 4 \bigoplus_{j_{L}=1}^{\infty}\left(j_{L}, j_{L} \pm \frac{1}{2}\right)^{ \pm} \oplus 4\left(\frac{1}{2}, 1\right)^{+} \\
\oplus 4\left(0, \frac{1}{2}\right)^{+} \oplus 84 \bigoplus_{j_{L}=\frac{1}{2}}^{\infty}\left(j_{L}, j_{L} \pm \frac{1}{2}\right)^{ \pm} \oplus 84\left(0, \frac{1}{2}\right)^{+} .
\end{gathered}
$$

- Chiral vectors:

$$
\begin{aligned}
5 \bigoplus_{j_{L}=1}^{\infty}\left(j_{L}, j_{L} \pm 1\right)^{\mp} & \oplus 5\left(\frac{1}{2}, \frac{3}{2}\right)^{-} \oplus 5(0,1)^{-} \oplus 21 \bigoplus_{j_{L}=1}^{\infty}\left(j_{L}, j_{L} \pm 1\right)^{ \pm} \\
& \oplus 21\left(\frac{1}{2}, \frac{3}{2}\right)^{+} \oplus 21(0,1)^{+} .
\end{aligned}
$$

The notation

$$
\left(j_{L}, j_{L} \pm \frac{1}{2}\right)^{\mp}=\left(j_{L}, j_{L}+\frac{1}{2}\right)^{-} \oplus\left(j_{L}, j_{L}-\frac{1}{2}\right)^{+}
$$


symbolizes two towers of KK modes. Furthermore, the three-dimensional fermions in the above spectrum are Dirac spinors and the chiral vectors are complex. As in the previous chapters the six-dimensional symplectic-Majorana and reality conditions are implemented by only considering the modes that have either $j_{R}^{3} \geq 0$ or $j_{L}^{3} \geq 0$, where $j_{L, R}^{3}$ are the eigenvalues of the generators of $\mathfrak{u}(1)_{L, R} \subset \mathfrak{s u}(2)_{L, R}$.

The KK spectrum on $S^{3} / \Gamma$ is given by the states that are invariant under the action of $\Gamma$ for $\Gamma=\mathbb{Z}_{m}$ and $\Gamma=\mathbb{D}_{m}^{*}$. As we found in appendix $3 . \mathrm{B}$ for $\Gamma=\mathbb{Z}_{m}$ the invariant states satisfy $j_{L}^{3}=\frac{1}{2} m k$ with $k \in \mathbb{Z}$. When we also apply the symplectic-Majorana and reality conditions, this gets refined to $k \in \mathbb{Z}_{\geq 0}$. The invariant representations under the action of $\mathbb{D}_{m}^{*}$ have $j_{L}^{3}=m k$ with $k \in \mathbb{Z}_{\geq 0}$. Applying the reality conditions in addition we find that we only keep states with $j_{R}^{3} \geq 0$.

We now need to sum the contribution of the individual modes to the $3 \mathrm{~d}$ ChernSimons terms, given in table 3.1, over the spectrum that we have just presented. We need to regulate the sums using zeta-function regularization to obtain a finite result. Specifically, we use the sums (3.83). The relation between the resulting coefficients $\alpha_{I}$ of the Chern-Simons terms and levels and central charges is then given by 3.85 .

Let us proceed with the calculation of the quantum corrections for the A- and D-series. We shall present the final results here, leaving a more detailed exposition to appendix 5.A

A-series. We must sum over the representations listed above with $j_{L}^{3}=\frac{1}{2} m k$, $k \in \mathbb{Z}_{\geq 0}$. As in chapter 3 we implement this by first summing over all states with $j_{L}^{3}=\frac{1}{2} m k$, that is over $j_{L}=\frac{1}{2} m k, \frac{1}{2} m k+1, \ldots$ and then performing the sum over $k \in \mathbb{Z}_{\geq 0}$, keeping in mind that we must regularize the summations using zeta-function regularization. This is performed explicitly in appendix 5.A, with the final results

$$
k_{L}^{\text {loop }}=0, \quad k_{R}^{\text {loop }}=2 m, \quad\left(c_{L}-c_{R}\right)^{\text {loop }}=12 m,
$$

up to order $\mathcal{O}(1)$ in the charges. Adding these results to the classical contributions (5.10), and using $\left|\mathbb{Z}_{m}\right|=m$, the central charges and levels to order $\mathcal{O}(1)$ in the charges are:

$$
k_{L}=\frac{1}{2} m C \cdot C, \quad k_{R}=\frac{1}{2} m C \cdot C+2 m, \quad c_{L}=c_{R}+12 m .
$$

D-series. As there is no left-moving current isometry in this case there is no left level, $k_{L}$, to compute. As in the A-series case we sum the contributions of the 
individual representations over the previously presented KK spectrum, this time with $j_{L}^{3}=m k, k \in \mathbb{Z}_{\geq 0}$ and $j_{R}^{3} \geq 0$. As we argued in section 4.4 this can effectively be done by taking the A-series results (5.13), sending $m \rightarrow 2 m$ and dividing the result by two. Therefore, the one-loop contributions up to order $\mathcal{O}(1)$ in the charges are:

$$
k_{R}^{\text {loop }}=2 m, \quad\left(c_{L}-c_{R}\right)^{\text {loop }}=12 m .
$$

Adding these to $(5.10)$ and using $\left|\mathbb{D}_{m}^{*}\right|=4 m$, the final results for the central charges and right-moving level up to and including linear terms in the charges are:

$$
k_{R}=2 m C \cdot C+2 m, \quad c_{L}=c_{R}+12 m .
$$

\subsubsection{Summary}

For the ease of the reader we conclude by collating the results of this section. In the subsequent section we shall compare the results obtained here with the microscopic computations conducted there.

For the case of ALE transverse space we have shown that the only contribution is from the classical reduction of the action performed in section 5.2.3. The results for the central charges and levels are

$$
\begin{aligned}
k_{L} & =\frac{1}{2}|\Gamma| \eta_{\alpha \beta} Q^{\alpha} Q^{\beta}=\frac{1}{2}|\Gamma| C \cdot C \quad \text { only for } \Gamma=\mathbb{Z}_{m}, \\
k_{R} & =\frac{1}{2}|\Gamma| C \cdot C, \\
c_{L} & =c_{R},
\end{aligned}
$$

where $|\Gamma|$ may be read off of table 4.1 .

In contrast, for the case of ALF transverse space we must also include, in addition to the classical contribution of section 5.2.3, the quantum corrections obtained in the preceding section. For the A-series we find 5

$$
k_{L}=\frac{1}{2} m C \cdot C, \quad k_{R}=\frac{1}{2} m C \cdot C+2 m, \quad c_{L}=c_{R}+12 m,
$$

whilst for the D-series we find:

$$
k_{R}=2 m C \cdot C+2 m, \quad c_{L}=c_{R}+12 m .
$$

All results presented here are up to linear terms in the charges, we do not include any $\mathcal{O}(1)$ terms.

\footnotetext{
${ }^{5}$ Note that the central charges and levels do not follow from the results of chapter 3 by specializing to $\mathrm{CY}_{3}=\mathrm{K} 3 \times \mathrm{T}^{2}$. This is due to the presence of non-trivial one-cycles that are not present in Calabi-Yau threefolds with $S U(3)$ holonomy.
} 


\subsection{Microscopics}

In the previous section we have computed the central charges and levels macroscopically; the content of this section is to reproduce these results from a microscopic computation. Concretely, we shall compute the central charges and levels of the $2 \mathrm{~d} \mathcal{N}=(0,4)$ SCFTs living on the strings considered previously. For the case of transverse ALE space we utilize the results of [183, 184 to determine the worldvolume theory on D3-branes probing an ADE singularity. By placing the resultant four-dimensional SCFTs on $\mathbb{R}^{1,1} \times C$ and performing a suitable topological twist $71,188,192,193$ we obtain $2 \mathrm{~d} \mathcal{N}=(0,4)$ SCFTs, whose central charges and levels we may compute via a spectrum computation and anomaly arguments. In the ALF case it is convenient to use a dual M-theory description for the A-series. However, for the ALF D-series case such a clean microscopic setup is lacking and the matching of the microscopic results to the macroscopic ones remains an open problem.

\subsubsection{Transverse ALE spaces}

We first consider the microscopic theory describing a D3-brane wrapping a curve $C$ inside $\mathrm{K} 3$ and probing the singular limit of an ALE space $\mathbb{C}^{2} / \Gamma$ transverse to the brane. The worldvolume theory of a D3-brane on $\mathbb{R}^{1,3}$ probing a transverse ADE singularity is obtained by performing a projection of $\mathcal{N}=4 U(|\Gamma|)$ super Yang-Mills. The resulting theories are $\mathcal{N}=2$ supersymmetric gauge theories with known Lagrangian description [183, 184]. Their gauge and hypermultiplet field content is summarized in table 5.1. From the table we can read off that the $4 \mathrm{~d}$ $\mathcal{N}=2$ theories all have $n_{H}=n_{V}=|\Gamma|$ hyper- and vector multiplets. Our ultimate interest is the $2 \mathrm{~d}$ IR theory arising from wrapping the D3-brane on a curve $C$ inside K3, preserving $\mathcal{N}=(0,4)$ supersymmetry ${ }^{6}$ In order to preserve supersymmetry in $2 \mathrm{~d}$ it is necessary to perform a topological twist. Before proceeding with performing the twist, counting fields in the $2 \mathrm{~d}$ theory and evaluating the central charges and levels, we first discuss the representations of the field content in the $4 \mathrm{~d}$ theory.

A $4 \mathrm{~d} \mathcal{N}=2$ SCFT admits an $S U(2)_{R} \times U(1)_{r}$ R-symmetry. The supercharges transform under the total symmetry group $S O(1,3)_{\ell} \times S U(2)_{R} \times U(1)_{r}$ in the representations

$$
Q_{\alpha I} \in(\mathbf{2}, \mathbf{1}, \mathbf{2})_{1}, \quad \tilde{Q}_{\dot{\alpha}}^{I} \in(\mathbf{1}, \mathbf{2}, \mathbf{2})_{-1}
$$

${ }^{6}$ Interesting proposals for the IR sigma model of the D1-D5 system probing the A-type ALE and ALF spaces were studied in 194 . 


\begin{tabular}{ccc}
\hline$\Gamma$ & Gauge multiplets & Hypermultiplets \\
\hline $\mathbb{Z}_{m}$ & $U(1)^{m}$ & $m \times(\mathbf{1}, \mathbf{1})$ \\
$\mathbb{D}_{m}^{*}$ & $U(1)^{4} \times U(2)^{m-1}$ & $4 \times(\mathbf{1}, \mathbf{2})+(m-2) \times(\mathbf{2}, \mathbf{2})$ \\
$\mathbb{T}^{*}$ & $U(1)^{3} \times U(2)^{3} \times U(3)$ & $3 \times(\mathbf{1}, \mathbf{2})+3 \times(\mathbf{2}, \mathbf{3})$ \\
$\mathbb{O}^{*}$ & $U(1)^{2} \times U(2)^{3} \times U(3)^{2} \times U(4)$ & $2 \times[(\mathbf{1}, \mathbf{2})+(\mathbf{2}, \mathbf{3})+(\mathbf{3}, \mathbf{4})]+(\mathbf{2}, \mathbf{4})$ \\
$\mathbb{I}^{*}$ & $U(1) \times U(2)^{2} \times U(3)^{2} \times$ & $(\mathbf{1}, \mathbf{2})+(\mathbf{2}, \mathbf{3})+(\mathbf{3}, \mathbf{4})+(\mathbf{4}, \mathbf{5})+$ \\
& $U(4)^{2} \times U(5) \times U(6)$ & $(\mathbf{5}, \mathbf{6})+(\mathbf{2}, \mathbf{4})+(\mathbf{4}, \mathbf{6})+(\mathbf{3}, \mathbf{6})$ \\
\hline
\end{tabular}

Table 5.1: $\mathcal{N}=2$ multiplets for the different quivers associated to the groups $\Gamma$.

Let us consider a generic $4 \mathrm{~d} \mathcal{N}=2 \mathrm{SCFT}$ with $n_{H} \mathcal{N}=2$ hypermultiplets and $n_{V}$ $\mathcal{N}=2$ vector multiplets. An $\mathcal{N}=2$ hypermultiplet has field content

$$
q^{I} \in(\mathbf{1}, \mathbf{1}, \mathbf{2})_{0}, \quad \psi_{\alpha}^{i=1,2} \in(\mathbf{2}, \mathbf{1}, \mathbf{1})_{-1}, \quad \tilde{\psi}_{\dot{\alpha}}^{i=1,2} \in(\mathbf{1}, \mathbf{2}, \mathbf{1})_{1},
$$

whilst an $\mathcal{N}=2$ vector multiplet contains the fields

$$
A_{\mu} \in(\mathbf{2}, \mathbf{2}, \mathbf{1})_{0}, \quad \lambda_{\alpha}^{I} \in(\mathbf{2}, \mathbf{1}, \mathbf{2})_{1}, \quad \tilde{\lambda}_{\dot{\alpha}}^{I} \in(\mathbf{1}, \mathbf{2}, \mathbf{2})_{-1}, \quad \Phi \in(\mathbf{1}, \mathbf{1}, \mathbf{1})_{2} .
$$

Note that in the case of the A-series we expect the $4 \mathrm{~d} \mathcal{N}=2$ SCFT to enjoy an additional $U(1)_{L}$ global symmetry. This flavor symmetry manifests itself in the D-brane picture as a $U(1)_{L}$ isometry of the transverse ALE space under which the supercharges are invariant, and is absent for the D- and E-series. The discussion of the assignment of $U(1)_{L}$ charges for the $\mathcal{N}=2$ fields is relegated to appendix 5.B They will appear later in this section when we give the final $2 \mathrm{~d}$ spectrum in table 5.2 .

Placing the $4 \mathrm{~d}$ theories on $\mathbb{R}^{1,1} \times C$ breaks Lorentz symmetry with the group decomposing as $S O(1,3)_{\ell} \rightarrow S O(1,1) \times U(1)_{C}$. This splitting leads to the following decompositions of the $S O(1,3)_{\ell}$ representations:

$$
\begin{aligned}
S O(1,3)_{\ell} & \rightarrow S O(1,1) \times U(1)_{C}, \\
(\mathbf{2}, \mathbf{2}) & \rightarrow \mathbf{1}_{2,0} \oplus \mathbf{1}_{-2,0} \oplus \mathbf{1}_{0,2} \oplus \mathbf{1}_{0,-2}, \\
(\mathbf{2}, \mathbf{1}) & \rightarrow \mathbf{1}_{1,1} \oplus \mathbf{1}_{-1,-1}, \\
(\mathbf{1}, \mathbf{2}) & \rightarrow \mathbf{1}_{1,-1} \oplus \mathbf{1}_{-1,1} .
\end{aligned}
$$


The decomposition of the supercharges under $S U(2)_{R} \times S O(1,1) \times U(1)_{C} \times U(1)_{r}$ is:

$$
\begin{aligned}
& S O(1,3)_{\ell} \times S U(2)_{R} \times U(1)_{r} \rightarrow S U(2)_{R} \times S O(1,1) \times U(1)_{C} \times U(1)_{r}, \\
& Q_{\alpha I} \in(\mathbf{2}, \mathbf{1}, \mathbf{2})_{1} \rightarrow \mathbf{2}_{1,1,1} \oplus \mathbf{2}_{-1,-1,1}, \\
& \tilde{Q}_{\dot{\alpha}}^{I} \in(\mathbf{1}, \mathbf{2}, \mathbf{2})_{-1} \quad \rightarrow \quad \mathbf{2}_{1,-1,-1} \oplus \mathbf{2}_{-1,1,-1} \text {. }
\end{aligned}
$$

In order to preserve $\mathcal{N}=(0,4)$ in the $2 \mathrm{~d}$ theory we must perform a topological twist with respect to $U(1)_{r}[71,188,192,193$. The unique twist preserving $\mathcal{N}=(0,4)$ in $2 \mathrm{~d}$, up to redefinition, is implemented by defining the generator of $U(1)_{C}^{\prime}$ to be

$$
T_{C}^{\prime}=\frac{1}{2}\left(T_{C}+T_{r}\right)
$$

In this way we obtain two singlet supercharges, both with negative chirality thereby giving $\mathcal{N}=(0,4)$ supersymmetry in $2 \mathrm{~d}$. Next consider the decomposition of the various matter fields after the twist. The fields of an $\mathcal{N}=2$ hypermultiplet decompose as:

$$
\begin{aligned}
S O(1,3)_{\ell} \times S U(2)_{R} \times U(1)_{r} & \rightarrow S U(2)_{R} \times S O(1,1) \times U(1)_{C}^{\prime} \times U(1)_{r}, \\
q^{I} \in(\mathbf{1}, \mathbf{1}, \mathbf{2})_{0} & \rightarrow \mathbf{2}_{0,0,0} \equiv q^{I} \\
\psi_{\alpha}^{i=1,2} \in(\mathbf{2}, \mathbf{1}, \mathbf{1})_{-1} & \rightarrow \mathbf{1}_{1,0,-1} \oplus \mathbf{1}_{-1,-1,-1} \equiv \psi_{+}^{i=1,2} \oplus \psi_{-}^{i=1,2}, \\
\tilde{\psi}_{\dot{\alpha}}^{i=1,2} \in(\mathbf{1}, \mathbf{2}, \mathbf{1})_{1} & \rightarrow \mathbf{1}_{1,0,1} \oplus \mathbf{1}_{-1,1,1} \equiv \tilde{\psi}_{+}^{i=1,2} \oplus \tilde{\psi}_{-}^{i=1,2} .
\end{aligned}
$$

Likewise a $4 \mathrm{~d} \mathcal{N}=2$ vector multiplet decomposes as:

$$
\begin{aligned}
S O(1,3)_{\ell} \times S U(2)_{R} \times U(1)_{r} \rightarrow & S U(2)_{R} \times S O(1,1) \times U(1)_{C}^{\prime} \times U(1)_{r}, \\
A_{\mu} \in(\mathbf{2}, \mathbf{2}, \mathbf{1})_{0} \rightarrow & \mathbf{1}_{2,0,0} \oplus \mathbf{1}_{-2,0,0} \oplus \mathbf{1}_{0,1,0} \oplus \mathbf{1}_{0,-1,0} \\
& \equiv v_{+} \oplus v_{-} \oplus a \oplus \tilde{a} \\
\lambda_{\alpha}^{I} \in(\mathbf{2}, \mathbf{1}, \mathbf{2})_{1} & \rightarrow \mathbf{2}_{1,1,1} \oplus \mathbf{2}_{-1,0,1} \equiv \lambda_{+}^{I} \oplus \lambda_{-}^{I} \\
\tilde{\lambda}_{\dot{\alpha}}^{I} \in(\mathbf{1}, \mathbf{2}, \mathbf{2})_{-1} & \rightarrow \mathbf{2}_{1,-1,-1} \oplus \mathbf{2}_{-1,0,-1} \equiv \tilde{\lambda}_{+}^{I} \oplus \tilde{\lambda}_{-}^{I} \\
\Phi \in(\mathbf{1}, \mathbf{1}, \mathbf{1})_{2} & \rightarrow \mathbf{1}_{0,1,2} \equiv \Phi
\end{aligned}
$$

Having determined the $2 \mathrm{~d}$ fields we now turn our attention to evaluating their multiplicities. The $2 \mathrm{~d}$ fields with $U(1)_{C}^{\prime}$ charges $Q_{C}^{\prime}= \pm 1$ have multiplicities

$$
\operatorname{dim} H^{0}\left(C, K_{C}\right)=\operatorname{dim} H^{0}\left(C, K_{C}^{-1}\right)=\operatorname{dim} H^{1}\left(C, \mathcal{O}_{C}\right)=g,
$$

whereas the states with charge $Q_{C}^{\prime}=0$ have multiplicity $\operatorname{dim} H^{0}\left(C, \mathcal{O}_{C}\right)=1$. The latter identity follows since the only holomorphic functions on a compact Riemann 
surface are constants. The full $2 \mathrm{~d}$ massless spectrum follows from the decompositions (5.26) and (5.27) coupled with the above discussion of the multiplicities, and is given in table 5.2 We have included in table 5.2 the $U(1)_{L}$ flavor charge which is only present for the A-series case. We remind the reader that the details of the $U(1)_{L}$ charge assignment may be found in appendix $5 . \mathrm{B}$

\begin{tabular}{ccc}
\hline Bosons & $S U(2)_{R} \times U(1)_{L}$ & Multiplicity \\
\hline$a, \tilde{a}, \Phi$ & $2 \times \mathbf{1}_{0}, 2 \times \mathbf{1}_{0}$ & $g$ \\
$q^{I}$ & $\mathbf{2}_{1}, \mathbf{2}_{-1}$ & 1 \\
$v_{+}, v_{-}$ & $2 \times \mathbf{1}_{0}$ & 1 \\
\hline Fermions & $g$ \\
\hline$\lambda_{+}^{I}, \tilde{\lambda}_{+}^{I}$ & $2 \times \mathbf{2}_{0}$ & 1 \\
$\psi_{+}^{1}, \psi_{+}^{2}, \tilde{\psi}_{+}^{1}, \tilde{\psi}_{+}^{2}$ & $2 \times \mathbf{1}_{1}, 2 \times \mathbf{1}_{-1}$ & $g$ \\
$----_{-}^{-}-\tilde{\psi}_{-}^{-}----$ & $--------\tilde{\psi}_{-}^{2}$ \\
$\psi_{-}^{1}, \psi_{-}^{2}, \tilde{\psi}_{-}^{1}, \tilde{\psi}_{-}^{2}$ & $2 \times \mathbf{1}_{1}, 2 \times \mathbf{1}_{-1}$ & 1 \\
$\lambda_{-}^{I}, \tilde{\lambda}_{-}^{I}$ & $2 \times \mathbf{2}_{0}$ &
\end{tabular}

Table 5.2: Spectrum of the $2 \mathrm{~d} \mathcal{N}=(0,4)$ theory. The $U(1)_{L}$ charges are only relevant for the A-series.

With the full $2 \mathrm{~d}$ spectrum in hand we may compute 't Hooft anomaly coefficients and use their relation to the central charges and levels, see the recent paper 70 in the context of $\mathcal{N}=(0,2)$ theories as an example. The gravitational anomaly is easily computed via

$$
c_{L}-c_{R}=\operatorname{tr}{ }_{\text {Weyl }} \gamma_{3}=0,
$$

where $\gamma_{3}$ is the chirality matrix in $2 \mathrm{~d}$ with eigenvalues \pm 1 and the sum is over all Weyl fermions.

The right level is (recall $n_{V}=n_{H}=|\Gamma|$ )

$$
\begin{aligned}
k_{R} & =\operatorname{tr} \operatorname{Weyl}\left(\gamma_{3} Q_{R}^{2}\right)=|\Gamma|\left[2 g \times\left[\left(\frac{1}{2}\right)^{2}+\left(-\frac{1}{2}\right)^{2}\right]-2 \times\left[\left(\frac{1}{2}\right)^{2}+\left(-\frac{1}{2}\right)^{2}\right]\right] \\
& =|\Gamma|(g-1)=\frac{1}{2}|\Gamma| C \cdot C,
\end{aligned}
$$

where $Q_{R}$ is the charge under the Cartan of $S U(2)_{R}$. In the final line we have used adjunction which implies $g=\frac{1}{2} C \cdot C+1$ in order to express the level in terms of $C \cdot C$. 
Proceeding in the same way to compute the 't Hooft anomaly associated to $U(1)_{L}$ we find 7

$$
k_{L}=-\operatorname{tr}_{\text {Weyl }}\left(\gamma_{3} Q_{L}^{2}\right)=|\Gamma|(g-1)=\frac{1}{2}|\Gamma| C \cdot C .
$$

The minus sign appearing in the definition of $k_{L}$, in contrast to the definition of $k_{R}$, arises as $U(1)_{L}$ is a left-moving current whilst $S U(2)_{R}$ is a right-moving current. Unitarity imposes that the anomalies are positive semi-definite, and the minus sign for the left-moving 't Hooft anomaly is included to 'cancel off' the minus sign arising from the chirality matrix. Our 't Hooft anomaly considerations thus give

$$
k_{R}=\frac{1}{2}|\Gamma| C \cdot C, \quad k_{L}=\frac{1}{2}|\Gamma| C \cdot C, \quad c_{L}-c_{R}=0,
$$

which precisely matches the result from the macroscopic computation (5.17).

We would now like to exploit the relation $c_{R}=6 k_{R}=3|\Gamma| C \cdot C$ dictated by supersymmetry to determine the central charges. However, this only holds if one can correctly identify the $S U(2)_{R}$ with the 2 d R-symmetry and as already noted in the introduction of this chapter, this does not work for the center of mass modes. This can be seen explicitly from table 5.2 The center of mass modes are given by $q^{I}, \psi_{+}^{1,2}$, $\tilde{\psi}_{+}^{1,2}, \lambda_{-}^{I}$ and $\tilde{\lambda}_{-}^{I}$. For example, it is easy to see that $\lambda_{-}^{I}$ and $\tilde{\lambda}_{-}^{I}$ are left-moving but transform under $S U(2)_{R}$ which is forbidden if it is the (right-moving) R-symmetry. One should repeat these computations ignoring the center of mass modes in the traces. This yields the following:

$$
c_{L}=c_{R}=6 k_{R}=3|\Gamma| C \cdot C+6|\Gamma|,
$$

which are the central charges of the theory without the center of mass modes and is consistent with the results in 195,196 for $|\Gamma|=1$.

The central charges also follow from the relations $c_{L, R}=N_{B}^{L, R}+\frac{1}{2} N_{F}^{L, R}$, where $N_{B}^{L, R}$ is the number of left- and right-moving bosonic degrees of freedom and $N_{F}^{L, R}$ the number of left- and right-moving fermionic degrees of freedom after extracting the center of mass degrees of freedom.

\subsubsection{Transverse ALF spaces}

We now turn to studying the microscopics of the wrapped D3-brane probing a transverse ALF space. As there is strictly speaking no known worldvolume theory

\footnotetext{
${ }^{7}$ We compute the coefficient with half-integer $U(1)_{L}$ charges, with the embedding $U(1)_{L} \subset S U(2)_{L}$ in mind.
} 
for this setting, unlike in the ALE case, we use a convenient duality frame to determine the central charges and levels. We first do this for the A-series where the ALF space is given by Taub-NUT space with NUT-charge $m$. Dualizing to an M5-brane picture allows us to compute the microscopic data. Unfortunately, for the D-series this method runs into difficulties which we shall explain in more detail below.

A-series. To obtain an M-theory picture we first T-dualize along the NUT-circle, obtaining a type IIA setup with a D4-brane wrapping $C$ and the NUT-circle (with inverted radius), and $m$ NS5-branes wrapping the aforementioned circle and the entire K3. Uplifting to M-theory, we obtain M-theory compactified on $X=\mathrm{K} 3 \times \mathrm{T}^{2}$ with an M5-brane wrapping $C \times \mathrm{T}^{2}$ and $m$ M5-branes wrapping K3. As explained in [75], this system can effectively be described by an M5-brane wrapping $\mathcal{P}=C \times \mathrm{T}^{2}+m \mathrm{~K} 3$. This is the well-studied MSW CFT, where the Calabi-Yau threefold has been specialized to $\mathrm{K} 3 \times \mathrm{T}^{2}$. This leads to the added complication that there now exist one-cycles in the geometry which were assumed not to be present in the original MSW setup, as the Calabi-Yau was taken to have $S U(3)$ holonomy. In order to determine the microscopic central charges we compute the bosonic and fermionic spectrum in the effective $2 \mathrm{~d} \mathcal{N}=(0,4)$ theory on the M5-brane. This setup was already studied in 60, 189, 197] before, but for convenience and completeness we review the main points here.

The worldvolume theory of the M5-brane is a $6 \mathrm{~d}(2,0)$ theory with a $(2,0)$ tensor multiplet containing a chiral two-form and five scalar fields. Three of the five scalars parametrize the center of mass motion of the string in the transverse $\mathbb{R}^{3}$, whereas the remaining two scalars parametrize the position of the curve $\mathcal{P}$ inside $X=\mathrm{K} 3 \times \mathrm{T}^{2}$. Additionally, one obtains $2 \mathrm{~d}$ scalar fields from reducing the chiral two-form along two-forms on $\mathcal{P}$. Due to the self-duality constraint there will be $b_{2}^{-}(\mathcal{P})$ left-moving, and $b_{2}^{+}(\mathcal{P})$ right-moving scalar fields in two dimensions, where $b_{2}^{ \pm}(\mathcal{P})$ denote the number of harmonic (anti-)self-dual two-forms on $\mathcal{P}$. In total, we find

$$
N_{B}^{L}=d_{\mathcal{P}}+3+b_{2}^{-}(\mathcal{P}), \quad N_{B}^{R}=d_{\mathcal{P}}+3+b_{2}^{+}(\mathcal{P})
$$

left- and right-moving bosons. The contribution from the two scalars parametrizing the motion of $\mathcal{P}$ inside $\mathrm{K} 3 \times \mathrm{T}^{2}$ is denoted by $d_{\mathcal{P}}$ and will be determined momentarily. The number of left- and right-moving fermions can be determined in the standard 
way by using the formulas

$$
\begin{aligned}
& N_{F}^{L}=4 h^{0,1}(\mathcal{P})=2 b_{1}(\mathcal{P})=2 b_{1}(X)=4 \\
& N_{F}^{R}=4\left(b_{0}(\mathcal{P})+h^{0,2}(\mathcal{P})\right)=4\left(b_{0}(X)+h^{0,2}(\mathcal{P})\right)=4+4 h^{0,2}(\mathcal{P}) .
\end{aligned}
$$

Supersymmetry in the right-moving sector implies that the number of right-moving bosons equals the number of right-moving fermions, allowing us to determine $d_{\mathcal{P}}$ via

$$
0=N_{B}^{R}-N_{F}^{R}=d_{\mathcal{P}}+b_{2}^{+}(\mathcal{P})-4 h^{0,2}(\mathcal{P})-1=d_{\mathcal{P}}-2 h^{0,2}(\mathcal{P}),
$$

where we have used that $b_{2}^{+}(\mathcal{P})=b_{2}(\mathcal{P})-b_{2}^{-}(\mathcal{P})$ and $b_{2}^{-}(\mathcal{P})=h^{1,1}(\mathcal{P})-1$. For the left- and right-moving central charges we find,

$$
c_{L}=b_{2}(\mathcal{P})+4, \quad c_{R}=3 b_{2}^{+}(\mathcal{P})+3 .
$$

We may evaluate these explicitly by rewriting the topological numbers in terms of integrals on $X=\mathrm{K} 3 \times \mathrm{T}^{2}$ as 45,60 .

$$
c_{L}=\int_{X}\left(\mathcal{P}^{3}+c_{2}(X) \wedge \mathcal{P}\right)+6, \quad c_{R}=\int_{X}\left(\mathcal{P}^{3}+\frac{1}{2} c_{2}(X) \wedge \mathcal{P}\right)+6 .
$$

These are straightforward to evaluate for the curve $\mathcal{P}$, giving the result: 8 ,

$$
c_{L}=3 m C \cdot C+24 m+6, \quad c_{R}=3 m C \cdot C+12 m+6 .
$$

Using $c_{R}=6 k_{R}$ this matches the macroscopic result 5.18 up to and including linear order terms in the charges. We expect that the left level can be computed by adapting the techniques in 75 and chapter 3 .

D-series. We saw above that the microscopics in the A-series' case is relatively clean once we have dualized to an M-theory picture. The D-series' case on the other hand, is not as clean cut. This is because the dual M-theory setup is no longer accessible with the standard MSW techniques. To see why, let us perform the analogous duality chain as in the A-series. Performing a T-duality along the fiber of the D-series ALF space to type IIA we obtain a D4-brane wrapping $\mathrm{S}^{1} \times C \times \mathrm{S}_{\mathrm{D}}^{1}$ from the D3-brane, $m$ NS5-branes wrapping $\mathrm{K} 3 \times \mathrm{S}^{1}$ and in addition a so called ON5-plane on top of the NS5-branes 198. An ON5-plane is the analogue of an

${ }^{8}$ When one calculates the central charges using 't Hooft anomalies and the relation $c_{R}=6 k_{R}$ one finds the results 5.39 up to order $\mathcal{O}(1)$ 60. The difference is again caused by subtleties in identifying $S U(2)_{R}$ with the R-symmetry. 
ordinary orientifold plane for the NS5-brane, and leads to orthogonal gauge groups when placed on top of the NS5-branes. Lifting the type IIA setup to M-theory the D4 lifts to an M5-brane wrapping $C \times \mathrm{T}^{2}$, the NS5s to M5s on K3 whilst the ON5-plane becomes an OM5 plane wrapping the K3. At low energies the system of M5-branes with an OM5-plane on top realizes the familiar D-type $(2,0)$ SCFT in the ADE classification. This is a very involved setup and does not directly lead itself to the application of MSW to compute the central charges due to the presence of the orientifold.

A second chain of dualities, which can also be applied to all the other cases considered in this chapter, is to first T-dualize along the circle wrapped by the D3-brane, leading to a type IIA setup of a D2-brane probing the ALE/ALF space. Uplifting to M-theory we obtain an M2-brane on the curve $C$ probing the ALE/ALF space. The problem has now been rephrased in terms of M2-brane counting for five-dimensional black holes. Note that as this duality frame exists for all the cases considered here, it is to some extent the most universal setup. In flat space there is a connection between the black hole partition function and the topological string partition function. It is natural to conjecture that a generalization of this connection would be a useful tool in understanding the counting of M2-branes in this setup.

Since the center of the ALF space looks like $\mathbb{C}^{2} / \mathbb{D}_{m}^{*}$ it is not unreasonable to expect that the leading order contribution to the central charges and level agrees with the corresponding D-series ALE space. The macroscopic results 5.19 confirm this expectation. Clearly it is desirable to have a better understanding of this case and to obtain a first principles derivation of the microscopic central charges and level.

\section{Appendices}

\section{A Summation of 6D to 3D one-loop corrections}

In this appendix we present the full and explicit computation of the one-loop contributions to the three-dimensional $\mathfrak{u}(1)_{L}, \mathfrak{s u}(2)_{R}$ and gravitational ChernSimons terms from integrating out the massive KK modes arising in the reduction on $S^{3} / \mathbb{Z}_{m}$. With these results, and a little further work, we may also obtain the results for the reduction on $\mathrm{S}^{3} / \mathbb{D}_{m}^{*}$. For this reason in this appendix we will almost exclusively consider the A-series case. The necessary adaptation to the D-series case will be explained in the final section 5. A.5 
To determine the corrections furnished by the massive KK modes we sum the contributions of individual modes (see table 3.1) over the full KK spectrum derived in section 5.2.4. These sums are very similar to the ones in appendix 3.D. As we did there, we must regularize the summations using zeta-function regularization. In particular we will use the regularized summations 3.119 .:

$$
\begin{aligned}
\sum_{j_{L}=\frac{1}{2} m k}^{\infty} 1 & =\frac{1}{2}-\frac{1}{2} m k, \quad \sum_{j_{L}=\frac{1}{2} m k}^{\infty} j_{L}=\frac{1}{24}\left(-2+6 k m-3 k^{2} m^{2}\right) \\
\sum_{j_{L}=\frac{1}{2} m k}^{\infty} j_{L}^{2} & =\frac{1}{24}\left(-2 k m+3 k^{2} m^{2}-k^{3} m^{3}\right)
\end{aligned}
$$

The sums are performed over the integers or half integers when $\frac{1}{2} m k$ is integer or half integer respectively. In fact the regularized summations we require are 3.120 :

$$
\begin{aligned}
\sum_{k=1}^{\infty} \sum_{j_{L}=\frac{1}{2} m k}^{\infty} 1=-\frac{1}{4}+\frac{m}{24}, & & \sum_{k=1}^{\infty} \sum_{j_{L}=\frac{1}{2} m k}^{\infty} j_{L}=\frac{1}{24}-\frac{m}{48} \\
\sum_{k=1}^{\infty} \sum_{j_{L}=\frac{1}{2} m k}^{\infty} j_{L}^{2}=-\frac{m^{3}}{24 \cdot 120}+\frac{m}{144}, & & \sum_{k=1}^{\infty} \sum_{j_{L}=\frac{1}{2} m k}^{\infty} k^{2}=-\frac{m}{240} .
\end{aligned}
$$

We now calculate the corrections $k_{L}^{\text {loop }}, k_{R}^{\text {loop }}$ and $\left(c_{L}-c_{R}\right)^{\text {loop }}$ separately. As described in the main text we implement the projection condition $j_{L}=\frac{1}{2} m k$ for $k \in \mathbb{Z}_{\geq 0}$ by first summing over the representations $j_{L}=\frac{1}{2} m k, \frac{1}{2} m k+1, \ldots$ and subsequently summing over $k$. The structure of the KK spectrum is such that we have to restrict to $m \geq 3$ and do the sums for $k=0$ separately. In the calculation of the one-loop contribution to $k_{L}, k_{R}$ and $c_{L}-c_{R}$ we therefore first sum over the representations relevant for $k=0$ and afterwards we sum the contributions for $k \neq 0$. We will not perform the computation for the special cases $m=1,2$ explicitly as they give the same result up to order $\mathcal{O}(1)$ as the formulas for $m \geq 3$ evaluated in $m=1,2$.

\section{A.1 Relevant spectrum}

The part of the spectrum that contributes to $k=0$ is given by

- Spin- $\frac{3}{2}$ :

$$
4 \bigoplus_{j_{L}=1}^{\infty}\left(j_{L}, j_{L} \pm \frac{1}{2}\right)^{\mp}
$$


- Spin- $\frac{1}{2}$ :

$$
\begin{gathered}
4 \bigoplus_{j_{L}=2}^{\infty}\left(j_{L}, j_{L} \pm \frac{3}{2}\right)^{\mp} \oplus 4 \bigoplus_{j_{L}=0}^{1}\left(j_{L}, j_{L}+\frac{3}{2}\right)^{-} \oplus 4 \bigoplus_{j_{L}=1}^{\infty}\left(j_{L}, j_{L} \pm \frac{1}{2}\right)^{ \pm} \\
\oplus 4\left(0, \frac{1}{2}\right)^{+} \oplus 84 \bigoplus_{j_{L}=1}^{\infty}\left(j_{L}, j_{L} \pm \frac{1}{2}\right)^{ \pm} \oplus 84\left(0, \frac{1}{2}\right)^{+}
\end{gathered}
$$

- Chiral vectors:

$$
5 \bigoplus_{j_{L}=1}^{\infty}\left(j_{L}, j_{L} \pm 1\right)^{\mp} \oplus 5(0,1)^{-} \oplus 21 \bigoplus_{j_{L}=1}^{\infty}\left(j_{L}, j_{L} \pm 1\right)^{ \pm} \oplus 21(0,1)^{+} .
$$

where all the sums are over the integers.

The vector representations $(0,1)^{-} \oplus 21(0,1)^{+}$are mapped to themselves by the reality condition on the six-dimensional tensors. We thus count their contribution with a factor of $\frac{1}{2}$.

When $k>0$, we find that $j_{L}^{3}=\frac{1}{2} m k \geq \frac{m}{2}$ such that for $m \geq 3$ the following part of the spectrum contributes:

- Spin- $\frac{3}{2}$ :

$$
4 \bigoplus_{j_{L}=\frac{1}{2} m k}^{\infty}\left(j_{L}, j_{L} \pm \frac{1}{2}\right)^{\mp}
$$

- Spin- $\frac{1}{2}$ :

$$
4 \bigoplus_{j_{L}=\frac{1}{2} m k}^{\infty}\left(j_{L}, j_{L} \pm \frac{3}{2}\right)^{\mp} \oplus 4 \bigoplus_{j_{L}=\frac{1}{2} m k}^{\infty}\left(j_{L}, j_{L} \pm \frac{1}{2}\right)^{ \pm} \oplus 84 \bigoplus_{j_{L}=\frac{1}{2} m k}^{\infty}\left(j_{L}, j_{L} \pm \frac{1}{2}\right)^{ \pm} .
$$

- Chiral vectors:

$$
5 \bigoplus_{j_{L}=\frac{1}{2} m k}^{\infty}\left(j_{L}, j_{L} \pm 1\right)^{\mp} \oplus 21 \bigoplus_{j_{L}=\frac{1}{2} m k}^{\infty}\left(j_{L}, j_{L} \pm 1\right)^{ \pm} .
$$

The sums are again with integer steps.

\section{A.2 Correction to the left level}

Note that the contribution from the $k=0$ modes is zero in this case as they come with an overall factor of $\left(j_{L}^{3}\right)^{2}$ which clearly vanishes in the $k=0$ case. We next 
consider, separately at first, the contributions for $k>0$ for the different kinds of fields before summing all the results. For the spin- $\frac{3}{2}$ fermions we find

$$
\begin{aligned}
\alpha_{L}^{(3 / 2)} & =4 \sum_{k=1}^{\infty} \sum_{j_{L}=\frac{1}{2} m k}^{\infty} \frac{3}{8 \pi}\left(\frac{1}{2} m k\right)^{2}\left[2\left(j_{L}-\frac{1}{2}\right)+1-2\left(j_{L}+\frac{1}{2}\right)-1\right] \\
& =-\frac{3 m^{2}}{4 \pi} \sum_{k=1}^{\infty} \sum_{j_{L}=\frac{1}{2} m k}^{\infty} k^{2}=\frac{1}{8 \pi} \frac{m^{3}}{40} .
\end{aligned}
$$

Likewise the spin- $\frac{1}{2}$ fermions contribute as

$$
\begin{aligned}
\alpha_{L}^{(1 / 2)} & =4 \sum_{k=1}^{\infty} \sum_{j_{L}=\frac{1}{2} m k}^{\infty} \frac{1}{8 \pi}\left(\frac{1}{2} m k\right)^{2}(-6+2+42) \\
& =\frac{19}{4 \pi} m^{2} \sum_{k=1}^{\infty} \sum_{j_{L}=\frac{1}{2} m k}^{\infty} k^{2}=-\frac{1}{8 \pi} \frac{19}{120} m^{3} .
\end{aligned}
$$

Finally, the vectors contribute as

$$
\alpha_{L}^{\text {(vect) }}=\sum_{k=1}^{\infty} \sum_{j_{L}=\frac{1}{2} m k}^{\infty} \frac{1}{4 \pi}\left(\frac{1}{2} m k\right)^{2}(20-84)=\frac{1}{8 \pi} \frac{2}{15} m^{3} .
$$

Adding 5.42, (5.43) and 5.44), it follows that the one-loop contribution to the left level vanishes:

$$
k_{L}^{\text {loop }}=8 \pi \cdot\left(\alpha_{L}^{(3 / 2)}+\alpha_{L}^{(1 / 2)}+\alpha_{L}^{(\text {vect })}\right)=0 .
$$

\section{A.3 Correction to the right level}

Let us now perform the analogous computation for the right level. We begin by studying the contribution from the $k=0$ modes which do not vanish in this case. The spin- $\frac{3}{2}$ fermions contribute as

$$
4 \sum_{j_{L}=1}^{\infty} \frac{1}{4 \pi}\left[\left(j_{L}+\frac{1}{2}\right)\left(j_{L}+\frac{3}{2}\right)\left(2 j_{L}+2\right)-\left(j_{L}-\frac{1}{2}\right)\left(j_{L}+\frac{1}{2}\right)\left(2 j_{L}\right)\right]=-\frac{5}{4 \pi}
$$


The infinite towers contained within the spin- $\frac{1}{2}$ fermion spectrum give

$$
\begin{gathered}
\frac{1}{3 \pi} \sum_{j_{L}=2}^{\infty}\left[\left(j_{L}+\frac{3}{2}\right)\left(j_{L}+\frac{5}{2}\right)\left(2 j_{L}+4\right)-\left(j_{L}-\frac{3}{2}\right)\left(j_{L}-\frac{1}{2}\right)\left(2 j_{L}-2\right)\right]=-\frac{83}{4 \pi}, \\
\frac{-1}{3 \pi}(1+21) \sum_{j_{L}=1}^{\infty}\left[\left(j_{L}+\frac{1}{2}\right)\left(j_{L}+\frac{3}{2}\right)\left(2 j_{L}+2\right)-\left(j_{L}-\frac{1}{2}\right)\left(j_{L}+\frac{1}{2}\right)\left(2 j_{L}\right)\right]=\frac{55}{6 \pi},
\end{gathered}
$$

whilst the isolated representations give

$$
-\frac{1}{3 \pi}\left[-\frac{3}{2} \cdot \frac{5}{2} \cdot 4-\frac{5}{2} \cdot \frac{7}{2} \cdot 6+(1+21) \cdot \frac{1}{2} \cdot \frac{3}{2} \cdot 2\right]=\frac{23}{2 \pi} .
$$

Finally, the infinite towers contained within the vector spectrum contribute

$$
(5-21) \sum_{j_{L}=1}^{\infty} \frac{1}{6 \pi}\left[-\left(j_{L}+1\right)\left(j_{L}+2\right)\left(2 j_{L}+3\right)+\left(j_{L}-1\right) j_{L}\left(2 j_{L}-1\right)\right]=-\frac{32}{3 \pi}
$$

and the isolated representations $5(0,1)^{-} \oplus 21(0,1)^{-}$give (note that they come with an extra factor of $\frac{1}{2}$ )

$$
-\frac{1}{2}(5-21) \cdot \frac{1}{6 \pi} \cdot 1 \cdot 2 \cdot 3=\frac{8}{\pi} .
$$

Enumerating all the contributions from the above $k=0$ results gives

$$
\alpha_{R}^{k=0}=-\frac{16}{4 \pi}
$$

We now turn to the evaluation of the $k>0$ contributions. For the spin- $\frac{3}{2}$ fermions the contribution is

$$
\begin{aligned}
\alpha_{R}^{(3 / 2)} & =4 \sum_{k=1}^{\infty} \sum_{j_{L}=\frac{1}{2} m k}^{\infty} \frac{1}{4 \pi}\left[\left(j_{L}+\frac{1}{2}\right)\left(j_{L}+\frac{3}{2}\right)\left(2 j_{L}+2\right)-\left(j_{L}-\frac{1}{2}\right)\left(j_{L}+\frac{1}{2}\right)\left(2 j_{L}\right)\right] \\
& =\frac{1}{\pi} \sum_{k=1}^{\infty} \sum_{j_{L}=\frac{1}{2} m k}^{\infty}\left(\frac{3}{2}+6 j_{L}+6 j_{L}^{2}\right)=\frac{1}{4 \pi}\left(-\frac{1}{2}-\frac{m}{12}-\frac{m^{3}}{120}\right) .
\end{aligned}
$$


Correspondingly, the spin- $\frac{1}{2}$ fermions give

$$
\begin{aligned}
\alpha_{R}^{(1 / 2)}= & 4 \sum_{k=1}^{\infty} \sum_{j_{L}=\frac{1}{2} m k}^{\infty} \frac{1}{12 \pi}\left[\left(j_{L}+\frac{3}{2}\right)\left(j_{L}+\frac{5}{2}\right)\left(2 j_{L}+4\right)\right. \\
& \left.-\left(j_{L}-\frac{3}{2}\right)\left(j_{L}-\frac{1}{2}\right)\left(2 j_{L}-2\right)\right] \\
& -4(1+21) \sum_{k=1}^{\infty} \sum_{j_{L}=\frac{1}{2} m k}^{\infty} \frac{1}{12 \pi}\left[\left(j_{L}+\frac{1}{2}\right)\left(j_{L}+\frac{3}{2}\right)\left(2 j_{L}+2\right)\right. \\
= & \frac{1}{4 \pi} \sum_{k=1}^{\infty} \sum_{j_{L}=\frac{1}{2} m k}^{\infty}\left(22+24 j_{L}+24 j_{L}^{2}\right)-\frac{22}{3 \pi} \sum_{k=1}^{\infty} \sum_{j_{L}=\frac{1}{2} m k}^{\infty}\left(\frac{3}{2}+6 j_{L}+6 j_{L}^{2}\right) \\
= & \frac{1}{4 \pi}\left(-\frac{5}{6}+\frac{43}{36} m+\frac{19}{360} m^{3}\right) .
\end{aligned}
$$

Lastly the vector spectrum provides the contribution

$$
\begin{aligned}
& \alpha_{R}^{(\text {vect })}=(21-5) \sum_{k=1}^{\infty} \sum_{j_{L}=\frac{1}{2} m k}^{\infty} \frac{1}{6 \pi}\left[\left(j_{L}+1\right)\left(j_{L}+2\right)\left(2 j_{L}+3\right)\right.\left.-\left(j_{L}-1\right) j_{L}\left(2 j_{L}-1\right)\right] \\
&=-\frac{16}{\pi} \sum_{k=1}^{\infty} \sum_{j_{L}=\frac{1}{2} m k}^{\infty}\left(1+2 j_{L}+2 j_{L}^{2}\right)=\frac{1}{4 \pi}\left(\frac{32}{3}-\frac{8}{9} m+\frac{2}{45} m^{3}\right) .
\end{aligned}
$$

Adding (5.51), 5.52), 5.53) and 5.54 the one-loop contribution to the right level is

$$
\begin{aligned}
k_{R}^{\text {loop }} & =4 \pi \cdot\left(\alpha_{R}^{k=0}+\alpha_{R}^{(3 / 2)}+\alpha_{R}^{(1 / 2)}+\alpha_{R}^{(\text {vect })}\right) \\
& =2 m-28 .
\end{aligned}
$$

\section{A.4 Correction to $c_{L}-c_{R}$}

As in the previous section we first calculate the $k=0$ contribution before proceeding to calculate the $k>0$ contributions. The spin- $\frac{3}{2}$ fermion, spin- $\frac{1}{2}$ fermion and vector 
representations give

$$
\begin{aligned}
4 \sum_{j_{L}=1}^{\infty} \frac{7}{64 \pi} \cdot 2 & =-\frac{7}{16 \pi}, \\
\frac{1}{48 \pi}\left[-\sum_{j_{L}=2}^{\infty} 6-4-6+\sum_{j_{L}=1}^{\infty} 2+2+21 \sum_{j_{L}=1}^{\infty} 2+42\right] & =\frac{7}{16 \pi}, \\
(21-5) \sum_{j_{L}=1}^{\infty} \frac{1}{48 \pi} \cdot 4+\frac{1}{2}(21-5) \frac{1}{48 \pi} \cdot 3 & =\frac{1}{6 \pi},
\end{aligned}
$$

respectively. We have again included an extra factor of $\frac{1}{2}$ for the isolated vector representations. The $k=0$ contribution from the above corrections is

$$
\alpha_{\text {grav }}^{k=0}=-\frac{16}{96 \pi} .
$$

The $k>0$ contribution of the spin- $\frac{3}{2}$ fermions equals

$$
\alpha_{\mathrm{grav}}^{(3 / 2)}=4 \sum_{k=1}^{\infty} \sum_{j_{L}=\frac{1}{2} m k}^{\infty} \frac{7}{64 \pi} \cdot 2=\frac{1}{96 \pi}\left(-21+\frac{7}{2} m\right),
$$

the spin- $\frac{1}{2}$ fermions contribute

$$
\alpha_{\mathrm{grav}}^{(1 / 2)}=4 \sum_{k=1}^{\infty} \sum_{j_{L}=\frac{1}{2} m k}^{\infty} \frac{1}{48 \cdot 4 \pi}(-6+2+42)=\frac{1}{96 \pi}\left(-19+\frac{19}{6} m\right),
$$

and finally the vector modes give

$$
\alpha_{\text {grav }}^{\text {(vect) }}=(19-5) \sum_{k=1}^{\infty} \sum_{j_{L}=\frac{1}{2} m k}^{\infty} \frac{1}{48 \pi} \cdot 4=\frac{1}{96 \pi}\left(32-\frac{16}{3} m\right) .
$$

The total one-loop correction for $c_{L}-c_{R}$, obtained by summing 5.57 , (5.58), (5.59) and 5.60 is

$$
\begin{aligned}
\left(c_{L}-c_{R}\right)^{\text {loop }} & =96 \pi \cdot\left(\alpha_{\text {grav }}^{k=0}+\alpha_{\text {grav }}^{(3 / 2)}+\alpha_{\text {grav }}^{(1 / 2)}+\alpha_{\text {grav }}^{(\text {vect })}\right) \\
& =12 m-88 .
\end{aligned}
$$

\section{A.5 $\mathbb{D}_{m}^{*}$ quantum corrections from $\mathbb{Z}_{m}$}

In the previous sections we have computed the one-loop corrections to the levels and gravitational central charge for the A-series. We must also compute the right 
level and gravitational central charge for the D-series. In principle one should be able to perform the equivalent computations of the previous sections, however in practice this is not the most efficient way to obtain the desired result. Instead we can use the observation that the summation in the D-series is equivalent, modulo the $j_{R}^{3} \geq 0$ condition, to the summations of the A-series with $m \rightarrow 2 m$. We may therefore use the previous results and then impose the $j_{R}^{3} \geq 0$ condition. As the contribution for a given $j_{R}^{3}$ within a fixed $j_{R}$ representation is the same for $j_{R}^{3}$ and $-j_{R}^{3}$ imposing $j_{R}^{3} \geq 0$ can be performed by halving the above result and adding in $\frac{1}{2}$ of the contribution from $j_{R}^{3}=0$. The latter contributions can be seen to be of order $\mathcal{O}(1)$ in the charges and therefore, as we are working to linear order, we may neglect this discrepancy and use the above shortcut. Performing the computation as outlined in the A-series section above for the D-series will result in the same linear order results. The final quantum corrections for the D-series are then

$$
k_{R}^{\text {loop }}=2 m, \quad\left(c_{L}-c_{R}\right)^{\text {loop }}=12 m .
$$

\section{B $U(1)_{L}$ charges of $\mathcal{N}=2$ fields}

In this appendix we discuss the $U(1)_{L}$ charges of the various fields in the $\mathcal{N}=2$ quivers associated to a transverse ALE space in the A-series. Geometrically, this symmetry is realized as a $U(1)_{L}$ isometry of the ALE space corresponding to the A-series. This isometry is absent for the D- and E-series such that this $U(1)_{L}$ flavor symmetry is not present in the latter field theories.

Recall that $4 \mathrm{~d} \mathcal{N}=2$ quiver gauge theories we are interested in can be obtained by a quotient of $\mathcal{N}=4$ super Yang-Mills. An $\mathcal{N}=4$ vector multiplet consists of a gauge field $A_{\mu}^{\mathcal{N}=4}$, six scalars $\varphi^{i}$ and fermions $\Psi_{\alpha}^{\mathcal{I}}, \tilde{\Psi}_{\dot{\alpha} \mathcal{I}}$, where $\mathcal{I}=1, \ldots, 4$ and $i=1, \ldots, 6$. They transform under $S O(1,3)_{\ell} \times S U(4)_{R}$ in the representations

$$
A_{\mu}^{\mathcal{N}=4} \in(\mathbf{2}, \mathbf{2}, \mathbf{1}), \quad \varphi^{i} \in(\mathbf{1}, \mathbf{1}, \mathbf{6}), \quad \Psi_{\alpha}^{\mathcal{I}} \in(\mathbf{2}, \mathbf{1}, \mathbf{4}), \quad \tilde{\Psi}_{\dot{\alpha} \mathcal{I}} \in(\mathbf{1}, \mathbf{2}, \overline{\mathbf{4}}),
$$

where $S U(4)_{R}$ is the R-symmetry of $\mathcal{N}=4$ SYM. An $\mathcal{N}=4$ vector multiplet can be decomposed into an $\mathcal{N}=2$ vector multiplet and a hypermultiplet. Identifying $S U(2)_{R} \times U(1)_{r} \subset S U(4)_{R}$ as the $\mathcal{N}=2$ R-symmetry, the $\mathcal{N}=4$ vector multiplet 
decomposes as

$$
\begin{aligned}
S O(1,3)_{\ell} \times S U(4)_{R} & \rightarrow S O(1,3)_{\ell} \times S U(2)_{R} \times U(1)_{r} \times U(1)_{L} \\
A_{\mu}^{\mathcal{N}=4} \in(\mathbf{2}, \mathbf{2}, \mathbf{1}) & \rightarrow(\mathbf{2}, \mathbf{2}, \mathbf{1})_{0,0} \\
\varphi^{i} \in(\mathbf{1}, \mathbf{1}, \mathbf{6}) & \rightarrow(\mathbf{1}, \mathbf{1}, \mathbf{1})_{2,0} \oplus(\mathbf{1}, \mathbf{1}, \mathbf{1})_{-2,0} \oplus(\mathbf{1}, \mathbf{1}, \mathbf{2})_{0,1} \oplus(\mathbf{1}, \mathbf{1}, \mathbf{2})_{0,-1} \\
\Psi_{\alpha \mathcal{I}} \in(\mathbf{2}, \mathbf{1}, \mathbf{4}) & \rightarrow(\mathbf{2}, \mathbf{1}, \mathbf{1})_{1_{1,1}} \oplus(\mathbf{2}, \mathbf{1}, \mathbf{1})_{1,-1} \oplus(\mathbf{2}, \mathbf{1}, \mathbf{2})_{-1,0} \\
\tilde{\Psi}_{\dot{\alpha}}^{\mathcal{I}} \in(\mathbf{1}, \mathbf{2}, \overline{\mathbf{4}}) & \rightarrow(\mathbf{1}, \mathbf{2}, \mathbf{1})_{-1,1} \oplus(\mathbf{1}, \mathbf{2}, \mathbf{1})_{-1,-1} \oplus(\mathbf{1}, \mathbf{2}, \mathbf{2})_{1,0} .
\end{aligned}
$$

The only states in the decomposition with non-vanishing $U(1)_{L}$ charge are the $(\mathbf{2}, \mathbf{1}, \mathbf{1})_{1, \pm, 1}$ state in the decomposition of $\Psi_{\alpha \mathcal{I}}$, the $(\mathbf{1}, \mathbf{2}, \mathbf{1})_{-1, \pm 1}$ state from $\tilde{\Psi}_{\dot{\alpha}}^{\mathcal{I}}$, and finally the $(\mathbf{1}, \mathbf{1}, \mathbf{2})_{0, \pm 1}$ states from the decomposition of $\varphi^{i}$. Since the fermionic states $(\mathbf{2}, \mathbf{1}, \mathbf{1})_{1, \pm, 1}$ and $(\mathbf{1}, \mathbf{2}, \mathbf{1})_{-1, \pm 1}$ are singlets under $S U(2)_{R}$, they are identified with the fermions in the $\mathcal{N}=2$ hypermultiplet. Similarly, since the states $(\mathbf{1}, \mathbf{1}, \mathbf{2})_{0, \pm 1}$ are scalar doublets under $S U(2)_{R}$, we conclude that they comprise the scalar degrees of freedom in the $\mathcal{N}=2$ hypermultiplet. In summary, the two scalars and the two fermions in an $\mathcal{N}=2$ hypermultiplet have opposite charges $Q_{L}= \pm 1$ under $U(1)_{L}$. On the other hand the fields in the vector multiplet are uncharged under this $U(1)_{L}$ global symmetry. This then leads to the $U(1)_{L}$ charges shown in table 5.2 Let us stress again that this additional global $U(1)_{L}$ symmetry only exists for the A-series. 


\section{Twisted $\mathcal{N}=1$ SCFTs and their $\mathrm{AdS}_{3}$ duals}

In this chapter, which is based on 4], we change gears a little bit and we construct new $\mathrm{AdS}_{3} / \mathrm{CFT}_{2}$ examples of the $\mathrm{AdS} / \mathrm{CFT}$ correspondence we discussed in section 1.4 However, as we will briefly discuss below, the geometry side of the new examples corresponds to the near-horizon geometry of black string solutions. We will compute the central charges of the CFTs as a check of the dualities. This corresponds to computing the entropy density of the black strings.

\subsection{Introduction}

Since the advent of AdS/CFT in 49 many examples of dual pairs have been conjectured. The first (and most studied) was $\mathrm{AdS}_{5} \times \mathrm{S}^{5}$ with five-form flux in type IIB and is proposed to be dual to four-dimensional $\mathcal{N}=4$ supersymmetric Yang-Mills [49]. We discussed the construction of this example in section 1.4 As we have seen there, the five-sphere can be replaced by an arbitrary five-dimensional Sasaki-Einstein manifold $Y_{5}$ and gives rise to $\mathrm{AdS}_{5} \times Y_{5}$ spaces dual to $4 \mathrm{~d} \mathcal{N}=1$ quiver gauge theories. In general, it is hard to find explicit metrics for these spaces and this makes it difficult to obtain evidence for new dualities $1^{1}$ Despite the difficulties, the infinite family of explicit Sasaki-Einstein metrics $Y_{5}=Y^{p, q}[52$ and its generalization $Y_{5}=L^{a, b, c} \sqrt[53]{ }$ have been discovered. These manifolds are toric and specified by coprime integers $p$ and $q$, and $a, b$ and $c$ respectively. As we have seen in section 1.4 the duality can be understood via its construction using D3-branes probing the Calabi-Yau cone singularity with base $Y_{5}$. The $4 \mathrm{~d}$ theory is the worldvolume theory on these D3-branes whilst $\mathrm{AdS}_{5} \times Y_{5}$ is the near-horizon geometry of the type IIB D3-brane solution.

Of course, one can also study the correspondence in lower dimensions. One way is to take the aforementioned four-dimensional theories and compactify them on a (smooth) Riemann surface $\Sigma_{g}$ with genus $g$ performing a topological twist

\footnotetext{
${ }^{1}$ One can make progress in understanding the gravity theory if one knows the topological data of the solution 199 200.
} 
to preserve supersymmetry. Additionally, background magnetic fluxes for the continuous flavour symmetries in the $4 \mathrm{~d}$ theory can be turned on. Since these fluxes are quantized, every $4 \mathrm{~d}$ theory leads to a discrete family of $2 \mathrm{~d}$ theories. In the IR one expects these theories to flow to a $2 \mathrm{~d}$ superconformal field theory (SCFT), however proving the existence of this flow is in general challenging. Assuming the existence of the SCFT one can compute its central charges and R-charges from UV data using the technique of c-extremization 69, 70 which is based on anomaly considerations. In this scenario we are not considering a conventional RG flow from a $2 \mathrm{~d}$ UV theory to the $2 \mathrm{~d}$ IR theory but instead we consider an $R G$ flow across dimensions. One can obtain evidence for the existence of the $2 \mathrm{~d}$ SCFT using holography by finding an explicit supergravity solution with the same central charges and R-charges. Employing the D3-brane picture, one expects the solution to correspond to the near-horizon geometry of a D3-brane compactified on the Riemann surface. This solution will be of the form $\operatorname{AdS}_{3} \times Y_{7}$ in which $Y_{7}$ is a fibration of the Sasaki-Einstein manifold $Y_{5}$ over the Riemann surface, with self-dual five-form flux sourced by the D3-branes. This solution can be thought of as the near-horizon geometry of a black string in an asymptotically $\mathrm{AdS}_{5} \times Y_{5}$ geometry. Computing the central charge of the dual CFT is related to computing the entropy density of this string.

The above has been successfully studied in the case that the $4 \mathrm{~d}$ parent theory is dual to $\mathrm{AdS}_{5} \times Y_{5}$ for $Y_{5}=\mathrm{S}^{5} 69$ 73, i.e. $\mathcal{N}=4$ super Yang-Mills, and for $Y_{5}=Y^{p, q}$ [4], see also 193 201]. For the latter case of $Y_{5}=Y^{p, q}$, local supergravity solutions have been found in [74] but their global regularity has not been studied there, see also 202,203. 2 However, even if explicit solutions are not known, one can still employ the recently found geometric dual of c-extremization 204206 . 3 For this technique one has to assume the existence of a solution and can compute its central charges and R-charges using only topological data characterizing the internal manifold. The equivalence to c-extremization has been proven in 212 for all toric quivers. Geometric c-extremization has been successfully applied to certain geometries including a subset of $Y_{5}=Y^{p, q}$ [205]. The dual of c-extremization from the perspective of gauged supergravity has been found in 213 and further investigated in [214].

In this chapter we extend the above and study compactifications of the $4 \mathrm{~d}$

\footnotetext{
${ }^{2}$ We have studied the regularity of the solutions in 74 in an appendix of $[4]$ on which this chapter is based. We will not present this in this thesis however.

${ }^{3}$ The closely related geometric dual of $\mathcal{I}$-extremization 207,208 was also put forward in 204 and further studied in 209 211.
} 
$L^{a, b, c}$ quiver gauge theories 215 217 on a Riemann surface $\Sigma_{g}$. Note that these theories include the previously studied $Y^{p, q}$ theories 218, as a special limit. We twist the $4 \mathrm{~d} L^{a, b, c}$ theories such that the resulting $2 \mathrm{~d}$ theories preserve $\mathcal{N}=(0,2)$ supersymmetry. On the field theory side we compute their central charges and R-charges using c-extremization. On the geometry side we compute these same charges, first by employing geometric c-extremization which has been developed for toric $Y_{5}$ in 205]. However, as mentioned above, to use this technique we must assume the existence of the explicit solution. We therefore construct and study solutions dual to $2 \mathrm{~d} \mathcal{N}=(0,2)$ SCFTs and match the central charges and R-charges to the expressions obtained via c-extremization.

The most important part of this chapter contains the construction of new solutions of the form $\mathrm{AdS}_{3} \times Y_{7}$ where $L^{a, b, c} \hookrightarrow Y_{7} \rightarrow \Sigma_{g}$. Such solutions are contained in the classification of supersymmetric $\mathrm{AdS}_{3}$ solutions of type IIB with only fiveform flux 187]. The ten-dimensional solutions are completely determined by a six-dimensional Kähler metric solving a master equation. We make a sufficiently general ansatz for this $6 \mathrm{~d}$ metric which is of cohomogeneity two and depends on three functions. The ansatz is motivated by the work of 219] in which the $L^{a, b, c}$ solutions of [53] were recovered using a Kähler orthotoric metric ansatz [220] in four dimensions. Their success motivates us to choose a fibration of the orthotoric metric over a Riemann surface for our ansatz. We extend these local metrics to globally well-defined metrics by studying their regularity without specifying the functions entering the ansatz. The resulting conditions from the regularity analysis place constraints on the roots of the functions in the ansatz and the first derivative of these functions evaluated there. Subsequently, we perform flux quantization, and compute the central charges and R-charges of baryonic operators while leaving the functions in the metric generic. Amazingly, the integrals one needs to compute can be integrated explicitly, even without solving the master equation. The resulting expressions only depend on the roots and first derivative of these functions. As a final step one can then determine the functions by solving the master equation. Plugging the result into the general expressions we obtain the various charges. The solution we find for the three functions in the ansatz solving the master equation do not contain enough free parameters to account for all the $2 \mathrm{~d}$ SCFTs. However, given these general expressions for the regularity conditions, the central charges and R-charges, it is simple to match them to the field theory once the most general solution has been found. The internal manifold of the solutions we find are examples of GK geometries 221]. These include previously known solutions in the literature of this form $70,74,202,221,224$ as special limits. For completeness note that 
other type IIB $\mathrm{AdS}_{3}$ solutions have been found and classified in $\left.131,132,225,229\right]$. In (massive) type IIA $\mathrm{AdS}_{3}$ solutions (and some type IIB solutions obtained via dualities) can be found in 226,230 235].

Despite the fact that the central charges and R-charges we find from the solution formally match the charges we compute from the twisted compactifications of the $L^{a, b, c}$ quiver gauge theories we have only found holographic dual pairs for the higher genus Riemann surfaces $\Sigma_{g>1}$. The gravity solutions we find for $\mathrm{S}^{2}$ and $\mathrm{T}^{2}$ Riemann surfaces are characterized by integers $a<0$ and $b, c>0$, whilst the $4 \mathrm{~d}$ field theory and therefore also the resulting $2 \mathrm{~d}$ theories are characterized by integers $a, b, c>0$. When $a>0\left(\mathrm{~S}^{2}\right.$ and $\mathrm{T}^{2}$ cases) at least one of the R-charges in the field theory result becomes negative, which is in contradiction with the chiral origin of the $2 \mathrm{~d}$ fields. It is therefore not clear what the $4 \mathrm{~d}$ theory compactified on $\mathrm{S}^{2}, \mathrm{~T}^{2}$ flows to in the IR. For the solutions containing a surface with $g>1$ we find gravity solutions with both $a>0$ and $a<0$. The former are the solutions which are holographically dual to $L^{a, b, c}$ quivers compactified on $\Sigma_{g>1}$. In the latter case where $a<0$ the central charges and R-charges from field theory and the corresponding solution again formally agree in spite of the different domains for the integers. Identifying the $2 \mathrm{~d}$ SCFTs dual to the $\mathrm{AdS}_{3}$ solutions with $a<0$ constructed in this chapter remains an open problem. This same phenomenon has been noted previously in 132,204,205 for solutions with internal space $\mathrm{T}^{2} \times Y^{p, q}$, $\mathrm{T}^{2} \times L^{a, b, c}$ and a specific fibration of $Y^{p, q}$ over $\mathrm{S}^{2}$. In the $Y^{p, q}$ cases the field theory is characterized by integers $p>q>0$, however the naive dual supergravity solutions, when known are instead characterized by integers $q>p>0.4$

The plan for the chapter is as follows. We first study the field theory side in section 6.2 where we review the technique of c-extremization and use it to compute the central charge and R-charges of the $2 \mathrm{~d} \mathcal{N}=(0,2)$ SCFTs. In section 6.3 we review geometric c-extremization and use it to compute the central charges and R-charges from the geometric side assuming the existence of the solutions. Subsequently in section 6.4 we turn our attention to finding these explicit solutions. We first study the regularity of our ansatz and compute expressions for the central charge, R-charges and flux quantization in terms of this general ansatz. Afterwards we solve the master equation and the conditions that follow from the regularity analysis to find globally regular solutions dual to 2d SCFTs. We use the general

\footnotetext{
${ }^{4}$ We also found this in $\left[4\right.$ for more general $Y^{p, q}$ fibrations over a Riemann surface $\Sigma_{g}$. We found that the solutions for Riemann surfaces with $g=0,1$ are also only globally regular for $q>p>0$. However, for $g>1$ this is not the case and solutions are valid for $p>q>0$. Therefore in the $g>1$ case, and only this case, there was evidence that the solutions and field theory are dual to each other.
} 
expressions to compute the central charge and R-charges and match with the field theory results. We conclude in section 6.5. Some technical material is relegated to appendices $6 . \mathrm{A}$ and $6 . \mathrm{B}$

\subsection{Twisted $\mathcal{N}=1$ field theories and c-extremization}

In this section we study the central charges and R-charges of baryonic operators of $2 \mathrm{~d} \mathcal{N}=(0,2)$ SCFTs arising from twisted compactifications of certain $4 \mathrm{~d} \mathcal{N}=1$ SCFTs on a compact genus $g$ Riemann surface $\Sigma_{g}$ from field theory. The $4 \mathrm{~d}$ parent theories are realized in string theory by $N$ D3-branes probing certain Calabi-Yau threefold singularities. The class of non-compact Calabi-Yau threefolds we consider here are metric cones over the five-dimensional Sasaki-Einstein manifolds $L^{a, b, c}$ discovered in $[53]$. This infinite family of metrics is labeled by a triplet of integers $a, b, c$ that can be chosen such that

$$
0<a \leq d \leq c \leq b, \quad \operatorname{gcd}(a, c)=\operatorname{gcd}(a, d)=\operatorname{gcd}(b, c)=\operatorname{gcd}(b, d)=1,
$$

where $d=a+b-c$. The $4 \mathrm{~d} \mathcal{N}=1$ field theories arising from this setup are quiver gauge theories 215217 . These field theories generically have two flavour symmetries $U(1)_{F_{1}} \times U(1)_{F_{2}}$, a baryonic $U(1)_{B}$ symmetry and in addition a $U(1)_{R}$ R-symmetry. We are interested in twisted compactifications of these $4 \mathrm{~d}$ theories on a Riemann surface $\Sigma_{g}$ with the most general flux configuration of the aforementioned global $U(1)$ symmetries preserving supersymmetry switched on. The IR fixed point (if it exists) is typically a strongly coupled $2 \mathrm{~d}$ SCFT which may not admit a Lagrangian description. Assuming the compactified theory flows to a $2 \mathrm{~d} \mathcal{N}=(0,2)$ SCFT, we shall compute the central charges and R-charges of baryonic operators of this $2 \mathrm{~d}$ theory. In order to make progress we will utilize the principle of cextremization put forward in 69]. This enables the computation of the central charges of $2 \mathrm{~d}$ SCFTs using UV data.

We begin by briefly reviewing c-extremization and the $4 \mathrm{~d} \mathcal{N}=1 L^{a, b, c}$ SCFTs in order to keep this chapter as self-contained as possible. Readers familiar with both may safely skip to section 6.2.2 in which we apply c-extremization to twisted compactifications of the $4 \mathrm{~d} L^{a, b, c}$ theories on a Riemann surface $\Sigma_{g}$ with constant curvature, turning on fluxes of global symmetries consistent with preserving supersymmetry. The trial central charge will be constructed and extremized with respect to the mixing parameters. While performing these computations in full generality is possible, we will only present the results of certain special cases which will be compared to their geometric counterparts later, since the results quickly become 
unwieldy. We have attached a Mathematica package to the publication [4] this chapter is based on in which the reader can find the results for the general case if they so wish.

\subsubsection{Topologically twisted 4d field theories and c-extremization}

In order to determine the (right-moving) central charge of the 2d SCFTs we employ the principle of c-extremization 69 . Similar to the more familiar a-maximization in $4 \mathrm{~d}$ [236], one constructs a trial central charge $c_{r}^{\text {trial }}\left(\varepsilon_{I}\right)$, a function depending on parameters $\varepsilon_{I}$ which parametrize the mixing of the UV R-symmetry with the remaining global symmetries. Given a $2 \mathrm{~d} \mathcal{N}=(0,2) \mathrm{UV}$ theory with R-symmetry $U(1)_{R}$ and global symmetries $U(1)_{I}$ we construct the trial central charge as follows First define the trial R-symmetry via the generator

$$
T_{R}^{\mathrm{trial}}=T_{R}+\sum_{I \neq R} \varepsilon_{I} T_{I} .
$$

The 't Hooft anomalies of the $2 \mathrm{~d}$ theory are encoded in the anomaly four-form polynomial $I_{4}$ of the 2 d theory. The trial central charge is defined via

$$
c_{r}^{\text {trial }}\left(\varepsilon_{I}\right)=3 k_{R R}^{\text {trial }},
$$

where $k_{R R}^{\text {trial }}$ is the quadratic 't Hooft anomaly coefficient of the trial R-symmetry in the anomaly polynomial

$$
I_{4} \supset \frac{1}{2} k_{R R}^{\text {trial }} c_{1}\left(F_{R}^{\text {trial }}\right) \wedge c_{1}\left(F_{R}^{\text {trial }}\right) .
$$

Here $F_{R}^{\text {trial }}$ is the background gauge field of the trial R-symmetry. The exact superconformal R-symmetry of the $2 \mathrm{~d}(0,2)$ SCFT is then obtained at the extremum of the trial central charge

$$
c_{R}=c_{r}^{\mathrm{trial}}\left(\varepsilon_{I}^{*}\right),\left.\quad \frac{\partial c_{r}^{\mathrm{trial}}\left(\varepsilon_{J}\right)}{\partial \varepsilon_{I}}\right|_{\varepsilon_{I}=\varepsilon_{I}^{*}}=0 .
$$

This extremization condition follows from the fact that the superconformal Rsymmetry at the IR fixed point has no mixed 't Hooft anomalies with any global symmetry, i.e. $k_{R I}=0$. The trial central charge evaluated at the extremum $c_{R}=c_{r}^{\text {trial }}\left(\varepsilon_{I}^{*}\right)$ is then the right-moving central charge of the SCFT. Furthermore the R-charges of operators can be computed by inserting $\varepsilon_{I}^{*}$ into the trial R-symmetry 6.2 . Let us note that the left-moving central charge can be computed from the result for the right-moving central charge and the gravitational anomaly of the $2 \mathrm{~d}$ 
SCFT. The difference of the left- and right-moving central charge is determined by the gravitational anomaly coefficient

$$
I_{4} \supset-\frac{c_{L}-c_{R}}{24} p_{1}\left(T M_{2}\right)
$$

in the anomaly polynomial. This contribution however, scales as $\mathcal{O}\left(N^{0}\right)$ in the theories under consideration and since we are only interested in the leading $\mathcal{O}\left(N^{2}\right)$ contribution in the large $N$ limit in this chapter, we shall not comment further on the subleading corrections.

The $4 \mathrm{~d} \mathcal{N}=1$ field theories which we wish to twist on a Riemann surface $\Sigma_{g}$ are quiver gauge theories with $S U(N)$ nodes and bifundamental matter linking the nodes. The gauge theory data necessary for our purposes are the bifundamental matter multiplets and gauginos together with their multiplicities and charges under the global $U(1)_{F_{1}} \times U(1)_{F_{2}} \times U(1)_{B} \times U(1)_{R}$ symmetry. The data of the six types of chiral bifundamentals included in the gauge theory, which are conventionally denoted by $Z, Y, U_{1,2}$ and $V_{1,2}$, and the vector multiplets, is summarized in table $6.1^{5}$ The integers $k, l$ are solutions of $c k+b l=1$ which exist by Bézout's identity.

\begin{tabular}{cccccc}
\hline Field & multiplicity & $U(1)_{F_{1}}$ & $U(1)_{F_{2}}$ & $U(1)_{B}$ & $U(1)_{R}$ \\
\hline$Z$ & $N^{2} a$ & 0 & $k$ & $b$ & -1 \\
$U_{2}$ & $N^{2} c$ & -1 & $-k-l$ & $-d$ & 0 \\
$Y$ & $N^{2} b$ & 1 & 0 & $a$ & -1 \\
$U_{1}$ & $N^{2} d$ & 0 & $l$ & $-c$ & 0 \\
$V_{1}$ & $N^{2}(b-c)$ & -1 & $-l$ & $c-a$ & 0 \\
$V_{2}$ & $N^{2}(c-a)$ & 0 & $k+l$ & $b-c$ & 0 \\
$\lambda$ & $\left(N^{2}-1\right)(a+b)$ & 0 & 0 & 0 & 1 \\
\hline
\end{tabular}

Table 6.1: Bifundamental matter and vector multiplets in the $L^{a, b, c}$ quiver and their charges. In this chapter we often refer to the first four multiplets with $\left(X_{1}, X_{2}, X_{3}, X_{4}\right)=$ $\left(Z, U_{2}, Y, U_{1}\right)$.

Note that our fiducial R-symmetry is not the R-charge assignment of the $4 \mathrm{~d}$ theory at the $4 \mathrm{~d}$ IR fixed point. Instead we choose a convenient set of R-charges such that the R-charge of the superpotential is two as usual.

Upon wrapping the $4 \mathrm{~d}$ theory on a genus $g$ Riemann surface $\Sigma_{g}$ we perform a partial topological twist to preserve $\mathcal{N}=(0,2)$ supersymmetry in $2 \mathrm{~d}$. We study

${ }^{5}$ The R-charge column is the (fiducial) R-charge $r$ of the fermionic component of the multiplet, the $\mathrm{R}$-charge of the boson is $r+1$. 
the most general twists involving flux for the R-symmetry, flavour, and baryonic symmetries on the Riemann surface. The total background gauge field configuration on the Riemann surface is

$$
\mathscr{F}_{\text {flux }}=\frac{\kappa}{2} \mathscr{R}_{g}+f_{1} \mathscr{F}_{1}+f_{2} \mathscr{F}_{2}+B \mathscr{F}_{B}
$$

where $\mathscr{F}_{A}=T_{A} \operatorname{dvol}\left(\Sigma_{g}\right)$ is the field strength associated to the symmetry $U(1)_{A}$ with its generator $T_{A}$. Here $\kappa$ is the (constant) normalized curvature of the Riemann surface with Ricci form $\mathscr{R}_{g}$. The volume form on the Riemann surface is normalized such that

$$
\operatorname{vol}\left(\Sigma_{g}\right)=\int_{\Sigma_{g}} \operatorname{dvol}\left(\Sigma_{g}\right)=2 \pi \eta_{g} \equiv\left\{\begin{array}{ll}
4 \pi|g-1|, & g \neq 1 \\
4 \pi, & g=1
\end{array} .\right.
$$

In order to construct the trial central charge for the compactified $4 \mathrm{~d}$ theories that we will study in this chapter, it is useful to work with the anomaly six-form polynomial of the parent $4 \mathrm{~d} \mathcal{N}=1$ theory. The anomaly polynomial for an $\mathcal{N}=1$ theory with global symmetries $U(1)_{I}$ and associated background field strengths $\mathcal{F}_{I}$ takes the form

$$
I_{6}=\frac{1}{3 !} k^{I J K} c_{1}\left(\mathcal{F}_{I}\right) \wedge c_{1}\left(\mathcal{F}_{J}\right) \wedge c_{1}\left(\mathcal{F}_{K}\right)-\frac{1}{24} k^{I} c_{1}\left(\mathcal{F}_{I}\right) \wedge p_{1}\left(T M_{4}\right),
$$

where $p_{1}\left(T M_{4}\right)$ is the first Pontryagin class of the four-manifold $M_{4}$ on which the $4 \mathrm{~d}$ theory is defined and $c_{1}\left(\mathcal{F}_{I}\right)$ are the first Chern classes associated with the global symmetries. The coefficients in the anomaly polynomial depend on the chiral spectrum of the $4 \mathrm{~d}$ theory via the cubic and linear 't Hooft anomalies

$$
k^{I J K}=\sum_{\text {Weyl fermions } \psi} Q_{\psi}^{I} Q_{\psi}^{J} Q_{\psi}^{K}, \quad k^{I}=\sum_{\text {Weyl fermions } \psi} Q_{\psi}^{I},
$$

where $Q_{\psi}^{I}$ is the charge of the fermion $\psi$ under $U(1)_{I}$. We can then compute the anomaly polynomial $I_{4}$ of the $2 \mathrm{~d} \mathcal{N}=(0,2)$ theory by integrating the $4 \mathrm{~d}$ one over the Riemann surface $\Sigma_{g}$. The dimensional reduction of the anomaly polynomial can be performed by including both the background flux and the mixing of the $2 \mathrm{~d}$ background gauge fields in the decomposition of the $4 \mathrm{~d}$ background gauge fields. The ansatz for the mixing of the UV R-symmetry with the remaining flavour symmetry can be parametrized by the generator

$$
T_{R}^{\text {trial }}=T_{R}+\varepsilon_{1} T_{F_{1}}+\varepsilon_{2} T_{F_{2}}+\varepsilon_{B} T_{B}
$$


where $\varepsilon_{i}, \varepsilon_{B}$ parametrize the mixing. The field strengths of $U(1)_{F_{1}} \times U(1)_{F_{2}} \times$ $U(1)_{B} \times U(1)_{R}$ including fluxes and mixing parameters are then identified as

$$
\begin{aligned}
\mathcal{F}_{R} & =F_{R}^{\text {trial }}+\frac{\kappa}{2} \mathscr{R}_{g}, \\
\mathcal{F}_{i} & =F_{i}+\varepsilon_{i} F_{R}^{\text {trial }}+f_{i} \mathscr{F}_{F_{i}}, \\
\mathcal{F}_{B} & =F_{B}+\varepsilon_{B} F_{R}^{\text {trial }}+B \mathscr{F}_{B},
\end{aligned}
$$

where now $F_{i}, F_{B}$ and $F_{R}^{\text {trial }}$ are the 2 d field strengths of the global symmetries and $F_{R}^{\text {trial }}$ is the field strength of the 2 d trial R-symmetry. The trial central charge is then given by the quadratic 't Hooft anomaly associated with the trial R-symmetry, i.e. $c_{r}^{\text {trial }}\left(\varepsilon_{i}, \varepsilon_{B}\right)=3 k_{R R}^{\text {trial }}$. The 't Hooft anomaly of the trial R-symmetry can now easily be computed by plugging the bundle decomposition 6.12 into the anomaly polynomial $I_{6}$ of the $4 \mathrm{~d}$ field theory and integrating the latter over the Riemann surface $\Sigma_{g}$, thereby obtaining the anomaly polynomial $I_{4}$ of the $2 \mathrm{~d}(0,2)$ theory. Reading off the coefficient

$$
I_{4}=\int_{\Sigma_{g}} I_{6} \supset \frac{1}{2} k_{R R}^{\text {trial }} c_{1}\left(F_{R}^{\text {trial }}\right) \wedge c_{1}\left(F_{R}^{\text {trial }}\right),
$$

one finds

$$
c_{r}^{\text {trial }}=-3 \eta_{g} \sum_{\text {fermions } \psi} \operatorname{mult}(\psi) T_{\psi}^{\mathrm{flux}}\left(Q_{R, \psi}^{\text {trial }}\right)^{2} .
$$

The sum runs over all $4 \mathrm{~d}$ fermions in the spectrum, mult $(\psi)$ is the multiplicity of $\psi$, $T_{\psi}^{\text {flux }}$ is the charge of the fermion under the background gauge field (6.7), and $Q_{R, \psi}^{\text {trial }}$ is the charge under the trial R-symmetry. Extremizing the trial central charge $c_{r}^{\text {trial }}\left(\varepsilon_{i}, \varepsilon_{B}\right)$ with respect to $\varepsilon_{I}=\left(\varepsilon_{i}, \varepsilon_{B}\right)$ and evaluating it at the extremum gives the value of the right-moving central charge at the (putative) IR fixed point.

\subsubsection{Central charges and R-charges from c-extremization}

It is now straightforward to compute the trial central charge for the $L^{a, b, c}$ field theories compactified on $\Sigma_{g}$ with fluxes using the data given in table 6.1 and the 
general formula for the trial central charge (6.14). One finds

$$
\begin{aligned}
c_{r}^{\text {trial }}\left(\varepsilon_{I}\right)= & -3 N^{2} \eta_{g}\left[(b-c)\left(B(c-a)-f_{1}-f_{2} l\right)\right. \\
& +(c-a)\left(B(b-c)+f_{2}(k+l)\right)\left((k+l) \varepsilon_{2}+(b-c) \varepsilon_{B}\right)^{2} \\
& +d\left(f_{2} l-B c\right)\left(l \varepsilon_{2}-c \varepsilon_{B}\right)^{2}-c\left(d B+f_{1}+f_{2}(k+l)\right) \\
& \times\left(\varepsilon_{1}+(k+l) \varepsilon_{2}+d \varepsilon_{B}\right)^{2}+b\left(a \varepsilon_{B}+\varepsilon_{1}-1\right)^{2}\left(a B+f_{1}-\frac{\kappa}{2}\right) \\
& \left.+a\left(b \varepsilon_{B}+k \varepsilon_{2}-1\right)^{2}\left(b B+f_{2} k-\frac{\kappa}{2}\right)+\frac{1}{2}(a+b) \kappa\right]
\end{aligned}
$$

for the most general twisted field theory and flux configuration on $\Sigma_{g}{ }^{6}$ It is possible to extremize the trial central charge 6.15 in this most general setting and evaluate the latter at the corresponding extremum. One thus obtains the right-moving central charge and R-charges of baryonic operators in the $2 \mathrm{~d}$ SCFT in full generality. However, since the full expressions are tedious, we refrain from giving the result for the most general case here and instead specialize to some interesting cases. The interested reader can find the most general result in a Mathematica file attached to the publication [4] this chapter is based on.

\subsubsection{Field theory c-extremization for $\Sigma_{g}=\mathrm{T}^{2}$}

We now present some explicit computations for the case when the $4 \mathrm{~d}$ parent theories are compactified on a torus $\mathrm{T}^{2}$. The formulas for the central charges and R-charges of the putative $2 \mathrm{~d}$ SCFT, to which the $4 \mathrm{~d}$ theory flows in the IR, are given and shall be compared to the geometric dual of c-extremization and supergravity in sections 6.3 .3 and 6.4 .6 respectively.

Purely baryonic flux. The first case we consider is the $4 \mathrm{~d} L^{a, b, c}$ field theory compactified on $\mathrm{T}^{2}$ with purely baryonic flux i.e. $f_{1}=f_{2}=0$. The values of the mixing parameters $\varepsilon_{I}$ extremizing the trial central charge 6.15 for this case can be easily computed and are given by

$$
\varepsilon_{1}^{*}=\frac{a(a-b+a k(b-c))}{(a-c)(c-b)}, \quad \varepsilon_{2}^{*}=\frac{a b}{c-a}, \quad \varepsilon_{B}^{*}=\frac{a(k(c-b)-1)}{(b-c)(c-a)} .
$$

\footnotetext{
${ }^{6}$ One can also derive this expression using the general results in 212 upon specializing the toric data to that of $L^{a, b, c}$.
} 
With these values for the mixing parameters one finds the right-moving central charge

$$
c_{R}=12 N^{2} B \frac{a b c(a+b-c)}{(a-c)(b-c)},
$$

and the R-charges of the baryonic operators are given by

$$
R\left[X_{1}\right]=R\left[X_{3}\right]=\frac{a b}{(a-c)(b-c)}, \quad R\left[X_{2}\right]=R\left[X_{4}\right]=-\frac{c d}{(a-c)(b-c)}
$$

Note that 6.18 are not well-defined R-charges for chiral multiplets as one of them necessarily is negative when $a, b, c$ satisfy 6.1. This has been noticed in 204 where it was shown from a gravity computation that this is a generic feature of these compactifications on a $\mathrm{T}^{2}$ with only baryonic flux. We can now examine for what domain of $a, b, c$ both the central charge and R-charges are positive. We wish to keep $0<d \leq c \leq b$ since the $Y^{p, q}$ limit is given by taking $a=p-q, b=p+q$ and $c=d=p$ for positive integers $p$ and $q$. Therefore the natural modification to make is to allow $a$ to take negative values.7 Indeed with these ranges we can simultaneously find a positive central charge and positive R-charges.

Fluxes $\boldsymbol{B} \propto \boldsymbol{f}_{\mathbf{2}}, \boldsymbol{f}_{\mathbf{1}}=\mathbf{0}$. Consider now a twisted compactification of the $L^{a, b, c}$ quiver gauge theories without $U(1)_{F_{1}}$ flux, and baryonic flux proportional to the $U(1)_{F_{2}}$ flux. Concretely, we choose a flux configuration where $B=-\frac{k}{b} f_{2}, f_{1}=0$. Extremizing the trial central charge 6.15 for these fluxes with respect to the mixing parameters $\varepsilon_{I}$ gives the extremal value of the central charge

$$
c_{R}=\frac{6 a k f_{2} N^{2}(-1+a k)[a+b-b c k+c(-2+c k)]}{b(-1+c k)+c(2-c k)+a^{2} k^{2}[c(2-c k)+b(-1+c k)]+a\left[-1-2 c k+c^{2} k^{2}+b k(1-c k)\right]},
$$

and the R-charges of baryonic operators are given by

$$
\begin{aligned}
& R\left[X_{1}\right]=-\frac{a b k(-1+a k)}{b(-1+c k)+c(2-c k)+a^{2} k^{2}[c(2-c k)+b(-1+c k)]+a\left[-1-2 c k+c^{2} k^{2}+b k(1-c k)\right]}, \\
& R\left[X_{2}\right]=\frac{(-1+a k)\left[a+b-b c k-a c^{2} k^{2}+a c k(1+b k)+c(-2+c k)\right]}{b(-1+c k)+c(2-c k)+a^{2} k^{2}[c(2-c k)+b(-1+c k)]+a\left[-1-2 c k+c^{2} k^{2}+b k(1-c k)\right]}, \\
& R\left[X_{3}\right]=\frac{a^{2} k(1+b k-c k)(-1+c k)}{b(-1+c k)+c(2-c k)+a^{2} k^{2}[c(2-c k)+b(-1+c k)]+a\left[-1-2 c k+c^{2} k^{2}+b k(1-c k)\right]}, \\
& R\left[X_{4}\right]=\frac{a^{2} c k^{2}+c(2-c k)+b(-1+c k)-a(1+c k)}{b(-1+c k)+c(2-c k)+a^{2} k^{2}[c(2-c k)+b(-1+c k)]+a\left[-1-2 c k+c^{2} k^{2}+b k(1-c k)\right]} .
\end{aligned}
$$

We find that at least one of the R-charges is negative when $a, b, c$ satisfy 6.1 .

${ }^{7}$ Technically the $Y^{p, q}$ limit does not fix the order of $c$ and $d$, however as in the $4 \mathrm{~d}$ theory we believe we can take $d \leq c$ without loss of generality. 


\subsubsection{Field theory c-extremization for $\Sigma_{g \neq 1}$}

We now move to twisted compactifications of the $L^{a, b, c}$ quiver gauge theories on a sphere and on higher genus Riemann surfaces. The parameter $\kappa \neq 0$ gives the distinction between $\Sigma_{g=0}=\mathrm{S}^{2}$ and $\Sigma_{g>1}$.

Baryonic flux and fixed flavour flux. For this field theory compactification we choose general baryonic flux given by $B$ and flavour fluxes with fixed values $f_{1}=-\frac{\kappa}{2} a k$ and $f_{2}=-\frac{\kappa}{2} b$. We note that there is nothing special in field theory for these values except that they give simple results. Extremizing the trial central charge we find

$$
c_{R}=\frac{6 a b c N^{2}(g-1)(k-2 B \kappa)^{2}(1+(a+b-c)(k-2 B \kappa))}{1+(k-2 B \kappa)(a+a(b-c)(k-2 B \kappa)-(b-c)(-1+c(k-2 B \kappa)))} .
$$

The R-charges at the conformal fixed point are given by

$$
\begin{aligned}
R\left[X_{1}\right] & =\frac{b(k-2 B \kappa)(a(k-2 B \kappa)+1)}{(k-2 B \kappa)(a(b-c)(k-2 B \kappa)+a-(b-c)(c(k-2 B \kappa)-1))+1}, \\
R\left[X_{2}\right] & =\frac{2-(a+b-c)(k-2 B \kappa)(c(k-2 B \kappa)-1)}{(k-2 B \kappa)(a(b-c)(k-2 B \kappa)+a-(b-c)(c(k-2 B \kappa)-1))+1}, \\
R\left[X_{3}\right] & =\frac{a(k-2 B \kappa)(b(k-2 B \kappa)+1)}{(k-2 B \kappa)(a(b-c)(k-2 B \kappa)+a-(b-c)(c(k-2 B \kappa)-1))+1}, \\
R\left[X_{4}\right] & =\frac{c(2 B \kappa-k)((a+b-c)(k-2 B \kappa)+1)}{(k-2 B \kappa)(a(b-c)(k-2 B \kappa)+a-(b-c)(c(k-2 B \kappa)-1))+1} .
\end{aligned}
$$

Here we also find that at least one of the R-charges is negative when $a, b, c$ satisfy 6.1. However, the central charge and R-charges take positive values when $a$ is negative and $0<d \leq c \leq b$.

\subsection{Geometric dual of c-extremization}

In this section we utilize the geometric dual of c-extremization 204,205 to compute the central charges and R-charges of the $L^{a, b, c}$ quiver theories reduced on a Riemann surface $\Sigma_{g}$ with constant curvature. We apply geometric c-extremization to geometries of the form $\mathrm{AdS}_{3} \times Y_{7}$, where $Y_{7}$ is a fibration of $L^{a, b, c}$ over $\Sigma_{g}$. We first describe the constraints on the geometry of the holographic duals of the $2 \mathrm{~d}$ SCFTs imposed by supersymmetry in section 6.3.1. We then briefly review the geometric c-extremization procedure in section 6.3.2 Again, the reader already familiar with these concepts may skip to section 6.3 .3 where we compute the central charges and R-charges applying this formalism. We subsequently compare the 
results obtained from the geometric dual to the field theory results of section 6.2 and find perfect agreement. This matching was already guaranteed by the recent proof of the equivalence of c-extremization and its geometric dual for all toric cases 212]. Despite this proof, the matching is a priori a formal matching since we have not proven the existence of the supergravity solution yet, this will be the subject of section 6.4 where we construct explicit solutions. The expressions we present in this section are essential for constructing the dictionary needed to compare these explicit solutions to the field theory.

\subsubsection{Holographic $\mathrm{AdS}_{3}$ duals with five-form flux}

In this chapter we are interested in the $2 \mathrm{~d}$ SCFTs arising from wrapping D3-branes on a constant curvature Riemann surface. The holographic duals of these SCFTs fall within the classification of [187]. There, the most general supersymmetric $\mathrm{AdS}_{3}$ solutions of type IIB supergravity with only five-form flux are shown to take the form 8

$$
\begin{aligned}
\mathrm{d} s^{2} & =L^{2} \mathrm{e}^{-\frac{w}{2}}\left(\mathrm{~d} s_{\mathrm{AdS}_{3}}^{2}+\frac{1}{4}(\mathrm{~d} z+P)^{2}+\mathrm{e}^{w} \mathrm{~d} s^{2}\left(\mathcal{M}_{6}\right)\right), \\
F_{5} & =(1+*) \mathrm{dvol}_{\mathrm{AdS}_{3}} \wedge\left(-2 J+\frac{1}{2} \mathrm{~d}\left[\mathrm{e}^{-w}(\mathrm{~d} z+P)\right]\right), \\
\mathrm{e}^{w} & =\frac{1}{8} R .
\end{aligned}
$$

The metric on $\mathrm{AdS}_{3}$ with unit radius is denoted by $\mathrm{d} s_{\mathrm{AdS}_{3}}^{2}$ and its corresponding volume form by $\mathrm{dvol}_{\mathrm{AdS}_{3}}$. Furthermore, $\mathcal{M}_{6}$ is a transverse Kähler metric with Kähler form $J$ and Ricci scalar $R$, satisfying the master equation

$$
\square R=\frac{1}{2} R^{2}-R_{i j} R^{i j}
$$

The one-form $P$ is the Ricci-form potential of the Kähler metric satisfying $\mathrm{d} P=\rho$ with $\rho$ the Ricci-form of $\mathcal{M}_{6}$. The scalar $w$ is determined in terms of $R$. Finally, $L$ is an overall dimensionful length scale. For a well-defined solution one must impose the flux quantization condition

$$
\frac{1}{\left(2 \pi \ell_{s}\right)^{4} g_{s}} \int_{\Sigma_{A}} F_{5}=N_{A} \in \mathbb{Z},
$$

where $\ell_{s}$ is the dimensionful string length, $g_{s}$ is the constant string coupling and $\Sigma_{A}$ form an integral basis for the free part of $H_{5}\left(Y_{7}, \mathbb{Z}\right)$. Note that the solution

${ }^{8}$ The five-form is rescaled with respect to the one in section 1.2 .2 as $F_{5}^{\text {here }}=g_{s} F_{5}^{\text {there }}$. 
6.23 has a unit norm Killing vector $\xi=2 \partial_{z}$, called the R-symmetry vector, which is dual to the R-symmetry of the SCFT and defines a foliation $\mathcal{F}_{\xi}$ of $Y_{7}$. Finally, the real cone over $Y_{7}$, i.e. $C\left(Y_{7}\right) \equiv \mathbb{R}_{>0} \times Y_{7}$ with metric

$$
\mathrm{d} s^{2}=\mathrm{d} r^{2}+r^{2} \mathrm{~d} s_{7}^{2},
$$

is complex but not Kähler. There is a nowhere-zero, closed and holomorphic (4,0)-form $\Psi$, which has R-charge two, i.e.

$$
\mathcal{L}_{\xi} \Psi=2 i \Psi
$$

hence $\xi$ is a holomorphic vector field.

\subsubsection{Geometric dual of c-extremization: general formalism}

Taking the SCFT as described in section 6.2 we will assume the existence of a dual solution satisfying the supersymmetry conditions and the master equation 6.26. Let us review how geometric c-extremization 204, 205 determines the central charges and R-charges of the dual SCFT. To apply this technique we take the solution off-shell which is done by only imposing the BPS-equations. We thus consider solutions of the form (6.23)- 6.25) satisfying (6.27). The solutions can then be put on-shell by additionally applying the five-form equation of motion 6.26 . The metric cone over the manifold $Y_{7}$, which we denote by $C\left(Y_{7}\right)$, is a complex cone with holomorphic volume form $\Psi$ and holomorphic $U(1)^{s}$ action generated by real holomorphic vector fields $\partial_{\varphi_{i}}, i=1, \ldots, s$. The R-symmetry vector $\xi$ is holomorphic and can be expanded as

$$
\xi=\sum_{i=1}^{s} b_{i} \partial_{\varphi_{i}} .
$$

We can choose a basis $\left\{\partial_{\varphi_{i}}\right\}_{i=1}^{s}$ such that $\Psi$ is only charged under $\partial_{\varphi_{1}}$, which by 6.29 implies that $b_{1}=2$. Once the vector field $\xi$ and thus foliation $\mathcal{F}_{\xi}$ is fixed, we have to choose a transverse Kähler metric with Kähler class $[J] \in H_{B}^{1,1}\left(\mathcal{F}_{\xi}\right)$. As remarked earlier, we must impose flux quantization on the off-shell solution over all compact five-cycles. The relevant part of the five-form flux is the part solely on the internal space and may be written as

$$
\left.F_{5}\right|_{\text {internal }}=\frac{1}{4}\left(4 \eta_{\mathrm{R}} \wedge \mathrm{d} \eta_{\mathrm{R}} \wedge J+\frac{1}{2} * \mathrm{~d} R\right),
$$

where $\eta_{R}=\frac{1}{2}(\mathrm{~d} z+P)$. With this expression the flux quantization conditions 6.27 are equivalent to

$$
\int_{\Sigma_{A}} \eta_{R} \wedge \rho \wedge J=\frac{2\left(2 \pi \ell_{s}\right)^{4} g_{s}}{L^{4}} N_{A}
$$


where $\Sigma_{A}$ are representative five-cycles on $Y_{7}$. This is only consistent in homology when

$$
\int_{Y_{7}} \eta_{R} \wedge \rho^{2} \wedge J=0
$$

is imposed in addition and $H^{2}\left(Y_{7}, \mathbb{R}\right) \cong H_{B}^{2}\left(\mathcal{F}_{\xi}\right) /[\rho]$, which holds for the fibered geometries $Y_{7}$ we consider here. The constraint equation 6.33 is equivalent to integrating the master equation 6.26 over $Y_{7}$. The off-shell central charge is defined by

$$
\mathscr{Z} \equiv \frac{3 L^{8}}{(2 \pi)^{6} g_{s}^{2} \ell_{s}^{8}} S_{\mathrm{SUSY}}=\frac{1}{2} \frac{3 L^{8}}{(2 \pi)^{6} g_{s}^{2} \ell_{s}^{8}} \int_{Y_{7}} \eta_{R} \wedge \rho \wedge J^{2} .
$$

The flux quantization condition 6.32, constraint equation 6.33 and trial central charge 6.34 only depend on the geometry via the choice of $\xi$ and the Kähler class $[J]$. The dual of c-extremization is given by extremizing 6.34 over these choices. The central charge of the dual SCFT is then given by

$$
c_{\text {sugra }}=\left.\mathscr{Z}\right|_{\text {on-shell }} \text {. }
$$

So far we have given a general description of geometric c-extremization, but now we will specialize this procedure to geometries that are fibrations of the form

$$
Y_{5} \hookrightarrow Y_{7} \rightarrow \Sigma_{g}
$$

where the fibers $Y_{5}$ are toric, i.e. they have a $U(1)^{3}$ isometry group generated by $\partial_{\varphi_{i}}, i=1,2,3$, with $\varphi_{i}$ having period $2 \pi$. Furthermore, we assume that the R-symmetry $\xi$ is tangent to $Y_{5}$ such that we can write

$$
\xi=\sum_{i=1}^{3} b_{i} \partial_{\varphi_{i}}
$$

Note that $\xi$ again defines a foliation $\mathcal{F}_{\xi}$ on $Y_{5}$ and we use the same notation for $\eta_{R}$ and its restriction to a fiber. We denote the transverse Kähler form for $\mathcal{F}_{\xi}$ by $\omega$. Since $C\left(Y_{7}\right)$ is complex, the cone $C\left(Y_{5}\right)$ is also complex with fixed complex structure. Moment map coordinates can be defined by

$$
\left.y_{i} \equiv \frac{1}{2} r^{2} \partial_{\varphi_{i}}\right\lrcorner \eta_{R}, \quad i=1,2,3,
$$

which are standard coordinates on $\mathbb{R}^{3}$. The image of the moment map is the convex polyhedral cone

$$
\mathcal{C}=\left\{\vec{y} \in \mathbb{R}^{3} \mid\left(\vec{y}, \vec{v}_{a}\right) \geq 0, \quad a=1, \ldots, f\right\}
$$


where $\vec{v} \in \mathbb{Z}^{3}$ are the inward pointing primitive normals to the facets, which we take to be ordered in an anti-clockwise direction. Furthermore, $f$ is the number of facets and $(\cdot, \cdot)$ is the Euclidean inner product. We assume that the cone $C\left(Y_{5}\right)$ admits a global holomorphic $(3,0)$-form such that a basis exists in which we can write $\vec{v}_{a}=\left(1, \vec{w}_{a}\right)$, for $\vec{w}_{a} \in \mathbb{Z}^{2}$. In this basis the holomorphic $(3,0)$-form has unit charge under $\partial_{\varphi_{1}}$ and is uncharged under $\partial_{\varphi_{2}}$ and $\partial_{\varphi_{3}}$, therefore, $b_{1}=2$. The Kähler class of the transverse metric of $Y_{5}[\omega] \in H_{B}^{2}\left(\mathcal{F}_{\xi}\right)$ is specified by parameters $\lambda_{a}, a=1, \ldots, f$ of which only $f-2$ are independent [237]. The Kähler class of the Riemann surface is determined by a parameter $A$. The fibration is specified by the first Chern numbers $\vec{n}=\left(n_{1}, n_{2}, n_{3}\right) \in \mathbb{Z}^{3}$ that are associated to the three $U(1)$ bundles over the Riemann surface. Using the transition functions of these bundles the manifold $Y_{5}$ is then fibered over $\Sigma_{g}$. Requiring the existence of the global (4,0)-form $\Psi$ on $C\left(Y_{7}\right)$ results in the condition

$$
n_{1}=2-2 g \text {, }
$$

which is the geometric equivalent of the field theory twist with the fiducial Rsymmetry. Flux quantization 6.32, the master equation 6.33 and trial central charge 6.34 can all be expressed using the so-called master volume

$$
\mathcal{V}\left(\vec{b} ;\left\{\lambda_{a}\right\}\right)=\frac{(2 \pi)^{3}}{2} \sum_{a=1}^{f} \lambda_{a} \frac{\lambda_{a-1}\left(\vec{v}_{a}, \vec{v}_{a+1}, \vec{b}\right)-\lambda_{a}\left(\vec{v}_{a-1}, \vec{v}_{a+1}, \vec{b}\right)+\lambda_{a+1}\left(\vec{v}_{a-1}, \vec{v}_{a}, \vec{b}\right)}{\left(\vec{v}_{a-1}, \vec{v}_{a}, \vec{b}\right)\left(\vec{v}_{a}, \vec{v}_{a+1}, \vec{b}\right)}
$$

and its derivatives, where $(\vec{v}, \vec{w}, \vec{u}) \equiv \operatorname{det}(\vec{v}, \vec{w}, \vec{u})$ denotes the determinant. The constraint equation 6.33 is now equal to

$$
A \sum_{a, b=1}^{f} \frac{\partial^{2} \mathcal{V}}{\partial \lambda_{a} \partial \lambda_{b}}-2 \pi n_{1} \sum_{a=1}^{f} \frac{\partial \mathcal{V}}{\partial \lambda_{a}}+2 \pi b_{1} \sum_{a=1}^{f} \sum_{i=1}^{3} n_{i} \frac{\partial^{2} \mathcal{V}}{\partial \lambda_{a} \partial b_{i}}=0
$$

and the flux quantization 6.32 becomes

$$
\begin{aligned}
\frac{2\left(2 \pi \ell_{s}\right)^{4} g_{s}}{L^{4}} N & =-\sum_{a=1}^{f} \frac{\partial \mathcal{V}}{\partial \lambda_{a}} \\
\frac{2\left(2 \pi \ell_{s}\right)^{4} g_{s}}{L^{4}} M_{a} & =\frac{A}{2 \pi} \sum_{b=1}^{f} \frac{\partial^{2} \mathcal{V}}{\partial \lambda_{a} \partial \lambda_{b}}+b_{1} \sum_{i=1}^{3} n_{i} \frac{\partial^{2} \mathcal{V}}{\partial \lambda_{a} \partial b_{i}} .
\end{aligned}
$$

The flux $N$ comes from taking the fiber $Y_{5}$ as five-cycle and the fluxes $M_{a}, a=$ $1, \ldots, f$ correspond to torus-invariant three-manifolds $S_{a} \subset Y_{5}$ fibered over $\Sigma_{g}$. The 
latter satisfy the three relations 205

$$
\sum_{a=1}^{f} v_{a}^{i} M_{a}=-n_{i} N, \quad i=1,2,3
$$

in agreement with the number of independent five-cycles $\operatorname{dim} H_{5}\left(Y_{5}, \mathbb{R}\right)=f-3$. The trial central charge (6.34) is given in terms of $\mathcal{V}$ by

$$
\mathscr{Z}=\frac{3 L^{8}}{(2 \pi)^{6} g_{s}^{2} \ell_{s}^{8}}\left(-A \sum_{a=1}^{f} \frac{\partial \mathcal{V}}{\partial \lambda_{a}}-2 \pi b_{1} \sum_{i=1}^{3} n_{i} \frac{\partial \mathcal{V}}{\partial b_{i}}\right)
$$

and depends after setting $b_{1}=2$ on two parameters since we have imposed $f$ relations on $f+2$ parameters. The value of $\mathscr{Z}$ at its extremal point is equal to $c_{\text {sugra }}$. The R-charges $R_{a}=R\left[S_{a}\right]$ of the baryonic operators dual to D3-branes wrapping the supersymmetric three-manifolds $S_{a} \subset Y_{7}$ on a fixed point of $\Sigma_{g}$ are given by 204

$$
R_{a}=R\left[S_{a}\right]=\frac{L^{4}}{(2 \pi)^{3} g_{s} \ell_{s}^{4}} \int_{S_{a}} \mathrm{e}^{-w} \operatorname{dvol}\left(S_{a}\right)=\frac{L^{4}}{(2 \pi)^{3} g_{s} \ell_{s}^{4}} \int_{S_{a}} \eta_{R} \wedge \omega
$$

where $\omega$ is the Kähler form of the transverse space. This may be rewritten in terms of the master volume as

$$
R_{a}=-\frac{L^{4}}{\left(2 \pi \ell_{s}\right)^{4} g_{s}} \frac{\partial \mathcal{V}}{\partial \lambda_{a}}
$$

Only $f-3$ of these are independent because of the relations 205

$$
\sum_{a=1}^{f} \vec{v}_{a} R_{a}=\vec{b} N
$$

Let us make a final remark that the central charges and R-charges can be expressed in field theory data using the transformation

$$
M_{a}=-\eta_{g} t\left[X_{a}\right] N
$$

which was conjectured in 205. In this relation, $t\left[X_{a}\right]$ are the charges of the bosonic fields $\left(X_{1}, X_{2}, X_{3}, X_{4}\right)=\left(Z, U_{2}, Y, U_{1}\right)$ of the quiver gauge theory with respect to the background gauge field 6.7). In the remainder of this section we use this transformation to compare c-extremization results with their geometric dual and provide further evidence for this identification of parameters. 


\subsubsection{Specifying to $Y_{5}=L^{a, b, c}$}

We now take $Y_{5}=L^{a, b, c}$ and compute the central charges and R-charges using the formalism reviewed in the previous section. We show how these results agree with the results of c-extremization on the field theory side. The Sasaki-Einstein metrics $L^{a, b, c}$ were constructed in $\sqrt[53]{53}$ and the inward pointing normal vectors are given by

$$
\vec{v}_{1}=(1,-a l, c), \quad \vec{v}_{2}=(1,0,0), \quad \vec{v}_{3}=(1,1,0), \quad \vec{v}_{4}=(1, a k, b) .
$$

For the computation we use the same steps and substitutions as in 205] for the $Y^{p, q}$ case; in particular we separate the cases with genus $g \neq 1$ and the case $g=1$. Using the normal vectors 6.51 we have an explicit expression for the volume function 6.41) and thus for the constraint equation 6.42 , the fluxes 6.43 and (6.44), the off-shell central charge (6.46) and the R-charges 6.48), where we impose $b_{1}=2$ after taking the derivatives. In the following we give explicit expressions for the central charge and R-charges for values of the parameters in which they simplify sufficiently and compare the results to the field theory computations shown in the previous sections. However, the interested reader can find the most general solutions to this extremization problem in a Mathematica notebook attached to the publication [4] that this chapter is based on, and can compare to the field theory results to their hearts' content.

\subsubsection{Geometric dual of c-extremization for $\Sigma_{g}=\mathrm{T}^{2}$}

For the genus one case we define rescaled fluxes by

$$
M_{a} \equiv m_{a} N
$$

and compute the dictionary translating between geometric and field theory parameters using 6.50):

$$
B=-\frac{m_{1}+k n_{3}}{2 b}, \quad f_{1}=\frac{n_{2}}{2}, \quad f_{2}=\frac{n_{3}}{2} .
$$

The latter relations will be useful once we wish to compare the results from this section to the ones from section 6.2.2.1

$\boldsymbol{n}_{\mathbf{2}}=\boldsymbol{n}_{\mathbf{3}}=\mathbf{0}$ : baryonic flux only. We first solve the constraint equation 6.42 the equation for $N(6.43)$ and the equation for $M_{1}$ in 6.44 in terms of $A, b_{3}$ and $\lambda_{4}$. After solving the remaining equations in 6.44 for $m_{2}, m_{3}$ and $m_{4}$ we find

$$
m_{2}=-\frac{(a+b-c) m_{1}}{b}, \quad m_{3}=\frac{a m_{1}}{b}, \quad m_{4}=-\frac{c m_{1}}{b} .
$$


As a check one can easily see that 6.45 is satisfied. The off-shell central charge 6.46 is then given by

$$
\mathscr{Z}=\frac{6 c(a+b-c)\left[2 a+b_{2}(b-c)\right]\left[-c b_{2}+a\left(-2+b_{2}+2 c k\right)\right] m_{1} N^{2}}{a[a+b-b c k+c(-2+c k)]^{2}},
$$

which can be shown to match with the trial central charge even off-shell. Extremizing with respect to $b_{2}$ we find the central charge

$$
c_{R}^{\text {geo }}=\frac{6 a c(a+b-c) m_{1} N^{2}}{(a-c)(c-b)} .
$$

Using that for $n_{2}=n_{3}=0$ 6.53 boils down to $m_{1}=-2 b B, f_{1}=f_{2}=0$, we find perfect agreement with the field theory result 6.17). The R-symmetry vector is given by $\vec{b}=\left(2, b_{2}^{*}, b_{3}^{*}\right)$ where

$$
b_{2}^{*}=\frac{a\left(a-c^{2} k+b(-1+c k)\right)}{(a-c)(c-b)}, \quad b_{3}^{*}=-\frac{b c}{a-c},
$$

are the values of the R-symmetry vector components at the extremum of the trial central charge. For the R-charges of baryonic operators one then finds that they can be written as

$$
\begin{array}{ll}
R_{1}=\frac{a b N}{(a-c)(b-c)}+b N \gamma, & R_{2}=\frac{(a+b-c) c N}{(a-c)(c-b)}-(a+b-c) N \gamma, \\
R_{3}=\frac{a b N}{(a-c)(b-c)}+a N \gamma, & R_{4}=\frac{(a+b-c) c N}{(a-c)(c-b)}-c N \gamma,
\end{array}
$$

where $\gamma$ is given by

$$
\begin{aligned}
\gamma= & \frac{c}{(b-c)(c-a)} \\
& +\frac{L^{4}\left[b^{2}\left(\lambda_{2}-\lambda_{1}\right)+b\left(2 a \lambda_{2}+c\left(\lambda_{1}-3 \lambda_{2}\right)\right)+(a-c)\left(a\left(\lambda_{2}-\lambda_{3}\right)-2 c \lambda_{2}\right)\right]}{2 \pi g_{s} \ell_{s}^{4} N a b c(a+b-c)} .
\end{aligned}
$$

As in 205] we can interpret $\gamma$ as parametrizing an undetermined transverse Kähler class which is not fixed by c-extremization. Note that one has to set $\gamma=0$ to identify the result 6.58 with the field theory c-extremization result 6.18 upon identifying $R_{a}=R\left[X_{a}\right] N$. Setting $\gamma=0$ is also implied by taking the $n_{2}, n_{3} \rightarrow 0$ limit of the $\mathrm{R}$-charges in the next paragraph. See 205] for a more extensive discussion of the parameter $\gamma$ in the context of c-extremization for $Y^{p, q}$. 
$\boldsymbol{n}_{\mathbf{2}}$ and $\boldsymbol{n}_{\mathbf{3}}$ not both zero. In this case the constraint equation 6.42 does not just depend on $b_{2}$ and $b_{3}$ but also on $\lambda_{i}$. Therefore we can follow a slightly different procedure than in the previous section and first solve for $A, \lambda_{3}$ and $\lambda_{4}$ using the constraint equation 6.42 , the equation for $N(6.43)$ and the equation for $M_{1}$ in 6.44. Subsequently we solve the remaining equations in 6.44 for $m_{2}, m_{3}$ and $m_{4}$. This results in

$$
\begin{aligned}
& m_{2}=\frac{c m_{1}+b\left(n_{2}-m_{1}\right)+n_{3}-a\left(m_{1}+k n_{3}\right)}{b} \\
& m_{3}=\frac{a m_{1}-b n_{2}+a k n_{3}}{b} \\
& m_{4}=-\frac{c m_{1}+n_{3}}{b}
\end{aligned}
$$

Following this, one can extremize the off-shell central charge with respect to $b_{2}$ and $b_{3}$. The resulting central charge and R-charges are equal to the field theory result using the transformation 6.53 . For the sake of readability we only show the expressions for the central charge and R-charges in a special case again, namely we take $m_{1}=n_{2}=0$. The R-symmetry vector is given by $\vec{b}=\left(2, b_{2}^{*}, b_{3}^{*}\right)$ with

$$
\begin{aligned}
b_{2}^{*} & =\frac{a k\left(a^{2} k+c(2-c k)+b(-1+c k)+a\left[-3+2 c k-c^{2} k^{2}+b k(-1+c k)\right]\right)}{b(-1+c k)+c(2-c k)+a^{2} k^{2}[c(2-c k)+b(-1+c k)]+a\left[-1-2 c k+c^{2} k^{2}+b k(1-c k)\right]}, \\
b_{3}^{*} & =\frac{b[-a+c(2-c k)+b(-1+c k)]}{b(-1+c k)+c(2-c k)+a^{2} k^{2}[c(2-c k)+b(-1+c k)]+a\left[-1-2 c k+c^{2} k^{2}+b k(1-c k)\right]} .
\end{aligned}
$$

The extremal value of the off-shell central charge is then given by

$$
c_{R}^{\mathrm{geo}}=\frac{3 a k(-1+a k)[a+b-b c k+c(-2+c k)] n_{3} N^{2}}{b(-1+c k)+c(2-c k)+a^{2} k^{2}[c(2-c k)+b(-1+c k)]+a\left[-1-2 c k+c^{2} k^{2}+b k(1-c k)\right]} .
$$

Using the translation from geometric to field theory parameters 6.53 adapted to this case, we find $B=-\frac{k}{b} f_{2}$ with $f_{2}=\frac{n_{3}}{2}$ and $f_{1}=0$. This is precisely the class of twisted compactifications studied in section 6.2.2.1 from the field theory perspective and again, we find perfect agreement between the field theory and geometric c-extremization results as expected [212]. Finally, the R-charges evaluated 
at the extremum are given by

$$
\begin{aligned}
& R_{1}=-\frac{a b k(-1+a k) N}{b(-1+c k)+c(2-c k)+a^{2} k^{2}[c(2-c k)+b(-1+c k)]+a\left[-1-2 c k+c^{2} k^{2}+b k(1-c k)\right]}, \\
& R_{2}=\frac{(-1+a k)\left[a+b-b c k-a c^{2} k^{2}+a c k(1+b k)+c(-2+c k)\right] N}{b(-1+c k)+c(2-c k)+a^{2} k^{2}[c(2-c k)+b(-1+c k)]+a\left[-1-2 c k+c^{2} k^{2}+b k(1-c k)\right]}, \\
& R_{3}=\frac{a^{2} k(1+b k-c k)(-1+c k) N}{b(-1+c k)+c(2-c k)+a^{2} k^{2}[c(2-c k)+b(-1+c k)]+a\left[-1-2 c k+c^{2} k^{2}+b k(1-c k)\right]}, \\
& R_{4}=\frac{a^{2} c k^{2}+c(2-c k)+b(-1+c k)-a(1+c k) N}{b(-1+c k)+c(2-c k)+a^{2} k^{2}[c(2-c k)+b(-1+c k)]+a\left[-1-2 c k+c^{2} k^{2}+b k(1-c k)\right]},
\end{aligned}
$$

which are once more found to be in agreement with the field theory computation upon identifying $R_{a}=R\left[X_{a}\right] N$.

\subsubsection{Geometric dual of c-extremization for $\Sigma_{g \neq 1}$}

In this case we rescale the fluxes $M_{a}$ and flavour flux parameters $n_{2}$ and $n_{3}$ according to

$$
M_{a} \equiv m_{a}(g-1) N, \quad n_{2} \equiv s_{2}(g-1), \quad n_{3} \equiv s_{3}(g-1) .
$$

We again solve for $A, \lambda_{3,4}$ and $m_{2,3,4}$ using 6.42 -6.44). We find

$$
\begin{aligned}
& m_{2}=\frac{c m_{1}+b\left(2-m_{1}+s_{2}\right)+s_{3}-a\left(m_{1}+k s_{3}\right)}{b}, \\
& m_{3}=\frac{a\left(m_{1}+k s_{3}\right)-b s_{2}}{b}, \\
& m_{4}=-\frac{c m_{1}+s_{3}}{b} .
\end{aligned}
$$

With the expressions for $A, \lambda_{3,4}, m_{2,3,4}$, the off-shell central charge 6.46 becomes a function of $g, N, m_{1}, s_{2,3}, b_{1,2}$. The dependence on $\lambda_{1}$ and $\lambda_{2}$ drops out, since only two of the $\lambda_{i}$ are independent. We then extremize the trial central charge with respect to $b_{2}$ and $b_{3}$ giving expressions for the central charge (6.46) and R-charges 6.48 for the most general flux configuration. Using the charge assignments in table 6.1 and 6.50 we can solve for $B$ and $f_{1,2}$ in terms of $m_{1}$ and $s_{2,3}$. We obtain the equalities

$$
B=\kappa \frac{m_{1}+k s_{3}+b k}{2 b}, \quad f_{1}=-\kappa \frac{s_{2}+a k}{2}, \quad f_{2}=-\kappa \frac{b+s_{3}}{2}
$$

for the dictionary between geometric and field theory data for the case $g \neq 1$. Substituting these relations into the central charge and R-charges of the field theory, we find that the results of c-extremization and its geometric dual agree. 
$s_{\mathbf{2}}=\boldsymbol{s}_{\mathbf{3}}=\mathbf{0}$. We now focus on the case where the parameters $s_{2,3}$ are both taken to be zero. The R-symmetry vector $\vec{b}=\left(2, b_{2}^{*}, b_{3}^{*}\right)$, is

$$
\begin{aligned}
b_{2}^{*} & =\frac{a\left[a-c^{2} k+b(-1+c k)\right] m_{1}^{2}}{b^{2}\left(m_{1}-1\right)-b(a-c)\left(m_{1}-1\right) m_{1}+(a-c) c m_{1}^{2}}, \\
b_{3}^{*} & =\frac{b(b-c) c m_{1}^{2}}{b^{2}\left(m_{1}-1\right)-b(a-c)\left(m_{1}-1\right) m_{1}+(a-c) c m_{1}^{2}} .
\end{aligned}
$$

The central charge is given by

$$
c_{R}^{\text {geo }}=\frac{6 a c(g-1) m_{1}^{2}\left[b\left(m_{1}-1\right)+(a-c) m_{1}\right] N^{2}}{b^{2}\left(m_{1}-1\right)-b(a-c)\left(m_{1}-1\right) m_{1}+(a-c) c m_{1}^{2}},
$$

and the R-charges are found to be

$$
\begin{aligned}
R_{1} & =\frac{b m_{1}\left(b-a m_{1}\right) N}{b^{2}\left(m_{1}-1\right)-b(a-c)\left(m_{1}-1\right) m_{1}+(a-c) c m_{1}^{2}}, \\
R_{2} & =\frac{\left[b^{2}\left(m_{1}-2\right)+b\left(a+c\left(m_{1}-1\right)\right) m_{1}+(a-c) c m_{1}^{2}\right] N}{b^{2}\left(m_{1}-1\right)-b(a-c)\left(m_{1}-1\right) m_{1}+(a-c) c m_{1}^{2}}, \\
R_{3} & =\frac{-a b\left(m_{1}-1\right) m_{1} N}{b^{2}\left(m_{1}-1\right)-b(a-c)\left(m_{1}-1\right) m_{1}+(a-c) c m_{1}^{2}}, \\
R_{4} & =\frac{-c m_{1}\left[b\left(1-m_{1}\right)+(c-a) m_{1}\right] N}{b^{2}\left(m_{1}-1\right)-b(a-c)\left(m_{1}-1\right) m_{1}+(a-c) c m_{1}^{2}} .
\end{aligned}
$$

From 6.66 we see that on the field theory side this corresponds to both nonvanishing baryonic flux $B=\kappa \frac{m_{1}+b k}{2 b}$ and flavour fluxes $f_{1}=-\frac{\kappa}{2} a k, f_{2}=-\frac{\kappa}{2} b$. Plugging these relations into the expressions for the central charge 6.68 and Rcharges 6.69 we recover the field theory results 6.21 and 6.22 as expected 212 .

\subsection{Explicit supergravity solutions}

Having computed central charges and R-charges using c-extremization and its geometric dual, in this section we will turn our attention to computing these charges with explicit supergravity solutions. In section 6.3.1 we saw that these solutions are characterized by solutions to the master equation 6.26. Our goal here is to find solutions that can be extended to be globally regular and whose central charges and R-charges can be matched to the results of the previous sections. In this endeavor we utilize an ansatz of cohomogeneity two which depends on four unknown functions. We argue that for our purposes we can constrain the specific form of two of the four functions without loss of generality and then solve the master 
equation 6.26 for the remaining two functions. Regularity of the solutions imposes additional conditions on these functions and as usual we impose flux quantization for the solutions to be well-defined string theory backgrounds. It transpires that it is possible to perform the integrals for the expressions for the fluxes, central charge and R-charges of these solutions without detailed knowledge of these two functions. This means we are not constrained to perform the analysis independently for all the different cases, but can obtain final expressions that simply require one to input the final solution. Finally using the explicit expressions of these functions, we compute the charges of the dual field theories and match them to expressions derived via c-extremization.

We begin by motivating the ansatz we use in section 6.4.1. In section 6.4.2 we then turn to examining the regularity of these metrics which imposes additional constraints on the solutions. Following this we impose flux quantization in section 6.4.3 and then in section 6.4.4 we derive general expressions for the central charges and R-charges. We solve the master equation 6.26) in section 6.4.5 and subsequently in section 6.4 .6 we solve the regularity conditions, compute the central charges and $\mathrm{R}$-charges and match the expressions of the previous sections.

\subsubsection{Ansatz}

We have seen in section 6.3.1 that the geometry is completely determined by the choice of Kähler metric $\mathrm{d} s^{2}\left(\mathcal{M}_{6}\right)$ satisfying 6.26). To proceed we must determine a suitable ansatz for the Kähler metric. We wish to describe the $L^{a, b, c}$ quiver theories compactified on a Riemann surface and therefore we demand that $Y_{7}$ is a fibration over a Riemann surface $Y_{5} \hookrightarrow Y_{7} \rightarrow \Sigma_{g}$, with $Y_{5}$ admitting a $U(1)^{3}$ isometry. One of the $U(1)$ 's in $Y_{5}$ will be identified with the R-symmetry vector, leaving two $U(1)$ 's in the ansatz for the Kähler base metric $\mathrm{d} s^{2}\left(\mathcal{M}_{6}\right)$. For the Kähler metric we shall modify the orthotoric Kähler metric 220 used in 219 to recover the $L^{a, b, c}$ metrics $[53]$ to include a general fibration over a Riemann surface. The ansatz we use is

$$
\begin{aligned}
\mathrm{d} s^{2}\left(\mathcal{M}_{6}\right) & =\mathcal{H}(\eta, \xi) \mathrm{d} s^{2}\left(\Sigma_{g}\right)+\frac{\mathcal{F}(\xi)}{\eta-\xi}\left(\mathrm{d} \phi+\partial_{\xi} \mathcal{L}(\eta, \xi) \mathrm{d} \psi+\partial_{\xi} \mathcal{H}(\eta, \xi) \mathcal{A}_{g}\right)^{2} \\
& +\frac{\eta-\xi}{\mathcal{F}(\xi)} \mathrm{d} \xi^{2}+\frac{\eta-\xi}{\mathcal{G}(\eta)} \mathrm{d} \eta^{2}+\frac{\mathcal{G}(\eta)}{\eta-\xi}\left(\mathrm{d} \phi+\partial_{\eta} \mathcal{L}(\eta, \xi) \mathrm{d} \psi+\partial_{\eta} \mathcal{H}(\eta, \xi) \mathcal{A}_{g}\right)^{2} .
\end{aligned}
$$


The metric $\mathrm{d} s^{2}\left(\Sigma_{g}\right)$ is the metric on a genus $g$ Riemann surface of constant curvature normalized to be $0, \pm 1$ depending on the genus ${ }^{9}$ The one-form $\mathcal{A}_{g}$ is the potential for the Kähler form of the Riemann surface and satisfies $\mathrm{d} \mathcal{A}_{g}=J_{g}$. Explicit expressions are given by

$$
\mathrm{d} s^{2}\left(\Sigma_{g}\right)=\frac{1}{1-\kappa x^{2}} \mathrm{~d} x^{2}+\left(1-\kappa x^{2}\right) \mathrm{d} y^{2}, \quad \mathcal{A}_{g}=x \mathrm{~d} y .
$$

In the remainder of this section we shall analyze the ansatz that we have chosen. First note that the function $\mathcal{H}$ should be positive. When $\mathcal{L}=\eta \xi$ and $\mathcal{H}$ is constant, the metric 6.70 is the direct product of the orthotoric metric and a Riemann surface. Lastly, observe that the ansatz 6.70) admits some 'gauge' transformations which leaves the internal manifold invariant. One can perform an overall rescaling of the Kähler metric by a constant parameter $\gamma^{2}$ whilst concurrently implementing the coordinate transformations

$$
(\eta, \xi, \phi, \psi) \rightarrow\left(\alpha \eta+\beta, \alpha \xi+\beta, \frac{\phi}{\alpha \gamma^{2}}, \psi\right)
$$

and redefining

$$
\begin{array}{ll}
\hat{\mathcal{F}}(\xi)=\frac{\mathcal{F}(\alpha \xi+\beta)}{\alpha^{3} \gamma^{2}}, & \hat{\mathcal{H}}(\eta, \xi)=\gamma^{2} \mathcal{H}(\alpha \eta+\beta, \alpha \xi+\beta), \\
\hat{\mathcal{G}}(\eta)=\frac{\mathcal{G}(\alpha \eta+\beta)}{\alpha^{3} \gamma^{2}}, & \hat{\mathcal{L}}(\eta, \xi)=\gamma^{2} \mathcal{L}(\alpha \eta+\beta, \alpha \xi+\beta),
\end{array}
$$

and preserve the form of the metric on the internal space. This symmetry will be used later to simplify the function $\mathcal{H}$ such that the master equation 6.26 becomes more tractable.

By construction the metric 6.70 admits a closed two-form given by

$$
J=\mathcal{H} J_{g}+\mathrm{d} \xi \wedge\left(\mathrm{d} \phi+\eta \mathrm{d} \psi+\partial_{\xi}(\mathcal{H}) \mathcal{A}_{g}\right)+\mathrm{d} \eta \wedge\left(\mathrm{d} \phi+\xi \mathrm{d} \psi+\partial_{\eta}(\mathcal{H}) \mathcal{A}_{g}\right) .
$$

However, it is not in general complex for arbitrary $\mathcal{H}$ and $\mathcal{L}$. We impose that the metric is complex in appendix 6.A which is equivalent to a set of second order non-linear partial differential equations given by 6.136 . We are unable to solve these conditions in general but if we additionally require that the universal twist solution ${ }^{10}$ can be obtained from our ansatz, i.e. by fixing $\mathcal{L}$, we find that the most

\footnotetext{
${ }^{9}$ For a Riemann surface with our normalization, the Ricci-form satisfies $\rho_{g}=R J_{g}=\kappa J_{g}$ where the last equality follows from our choice of normalization of the metric. For $g \neq 1$ the Riemann-Roch theorem implies that the volume of the Riemann surface satisfies $\operatorname{vol}\left(\Sigma_{g}\right)=4 \pi|g-1|$.

${ }^{10}$ This is the standard topological twist solution when wrapping on $\Sigma_{g>1}$ and is obtained by taking a direct product of the orthotoric metric and the Riemann surface.
} 
general solution is given by

$$
\mathcal{H}(\eta, \xi)=\alpha_{0}+\alpha_{1}(\eta+\xi)+\alpha_{2} \eta \xi, \quad \mathcal{L}(\eta, \xi)=\eta \xi
$$

with $\mathcal{F}$ and $\mathcal{G}$ still undetermined. For the time being we shall refrain from using the explicit solution for $\mathcal{H}$ in order to keep expressions as compact as possible.

Using the expression for $\mathcal{L}$ the Ricci scalar of the metric is easily computed to be

$$
R=\frac{2 \kappa(\xi-\eta)+\mathcal{H}\left(\mathcal{F}^{\prime \prime}(\xi)+\mathcal{G}^{\prime \prime}(\eta)\right)+2\left(\mathcal{F}^{\prime}(\xi) \partial_{\xi} \mathcal{H}+\mathcal{G}^{\prime}(\eta) \partial_{\eta} \mathcal{H}\right)+3\left(\mathcal{F}(\xi) \partial_{\xi}^{2} \mathcal{H}+\mathcal{G}(\eta) \partial_{\eta}^{2} \mathcal{H}\right)}{(\xi-\eta) \mathcal{H}}
$$

whilst the Ricci-form connection is

$$
P=P_{g}-\frac{\partial_{\xi}(\mathcal{F H})}{2(\eta-\xi) \mathcal{H}}\left(\mathrm{d} \phi+\eta \mathrm{d} \psi+\partial_{\xi}(\mathcal{H}) \mathcal{A}_{g}\right)-\frac{\partial_{\eta}(\mathcal{G H})}{2(\eta-\xi) \mathcal{H}}\left(\mathrm{d} \phi+\xi \mathrm{d} \psi+\partial_{\eta}(\mathcal{H}) \mathcal{A}_{g}\right)
$$

Here $P_{g}$ is the Ricci-form connection on the Riemann surface. The full tendimensional solution follows from using 6.23 - 6.25 and the explicit values of $\mathcal{F}$ and $\mathcal{G}$ given later.

\subsubsection{Regularity analysis}

Before proceeding with determining the explicit solutions we shall present a preliminary analysis of the global regularity of the full seven-dimensional metric. By 6.25 we are allowed to restrict to the seven-dimensional solution instead of the full ten-dimensional one if we also require that the Ricci scalar is strictly positive. As the remaining space is a fibration of the form $Y_{5} \hookrightarrow Y_{7} \rightarrow \Sigma_{g}$ we may first check the global regularity of $Y_{5}$ before checking the fibration over $\Sigma_{g}$. The metric on $Y_{5}$ is given by

$$
\begin{aligned}
\mathrm{d} s^{2}\left(Y_{5}\right)= & \frac{1}{4}\left(\mathrm{~d} z-\frac{1}{2(\eta-\xi)}\left[\left(\mathcal{F}^{\prime}(\xi)+\frac{\mathcal{F} \partial_{\xi} \mathcal{H}}{\mathcal{H}}\right)(\mathrm{d} \phi+\eta \mathrm{d} \psi)\right.\right. \\
& \left.\left.+\left(\mathcal{G}^{\prime}(\eta)+\frac{\mathcal{G}(\eta) \partial_{\eta} \mathcal{H}}{\mathcal{H}}\right)(\mathrm{d} \phi+\xi \mathrm{d} \psi)\right]\right)^{2} \\
+ & \frac{R}{8}\left[\frac{\eta-\xi}{\mathcal{F}(\xi)} \mathrm{d} \xi^{2}+\frac{\mathcal{F}(\xi)}{\eta-\xi}(\mathrm{d} \phi+\eta \mathrm{d} \psi)^{2}+\frac{\eta-\xi}{\mathcal{G}(\eta)} \mathrm{d} \eta^{2}+\frac{\mathcal{G}(\eta)}{\eta-\xi}(\mathrm{d} \phi+\xi \mathrm{d} \psi)^{2}\right] .
\end{aligned}
$$

To make the metric compact we must determine suitable ranges for the $\eta$ and $\xi$ coordinates, which are fixed by finding two roots of the functions $\mathcal{G}$ and $\mathcal{F}$ 
respectively. Moreover we require that $\eta-\xi$ is non-vanishing everywhere ${ }^{11}$ Without loss of generality we may take $\eta>\xi$ everywhere and consequently we require that both $\mathcal{F}$ and $\mathcal{G}$ are positive in their respective domains, i.e. they admit a local maximum between the two roots. The other case is simply a relabeling $\eta \leftrightarrow \xi$ and $\mathcal{G} \leftrightarrow-\mathcal{F}$. Moreover since we must impose that the function $\mathcal{H}$ is strictly positive we take without loss of generality that $0<\xi<\eta$, the other choice can be recovered by the coordinate transformation $(\eta, \xi) \rightarrow(-\eta,-\xi)$. We denote the roots by $\xi_{ \pm}$ and $\eta_{ \pm}$for $\mathcal{F}$ and $\mathcal{G}$ respectively with the subscript denoting the larger (smaller) of the two roots.

Consider now the metric at one of the roots. At each root the metric has a two-dimensional surface which degenerates. In the language of toric geometry this is equivalent to one of the $U(1)$ 's shrinking on the facet of the polytope. This $U(1)$ corresponds to a specific Killing vector. Like in the classic example of a round $\mathrm{S}^{2}$, requiring that the surface degenerates smoothly and locally looks like flat space imposes constraints on the period of the aforementioned Killing vector. The by now well established method for performing the regularity analysis, is to normalize the surface gravity

$$
\kappa_{\text {grav }}^{2}=\frac{\partial_{\mu}|V|^{2} \partial^{\mu}|V|^{2}}{4|V|^{2}},
$$

of the degenerating Killing vector $V$ to be unity on the degeneration surface, see 53 . The metric on $Y_{5}$ has a $U(1)^{3}$ isometry group. The three commuting Killing vectors are $\partial_{z}, \partial_{\psi}, \partial_{\phi}$ and linear combinations of these three Killing vectors degenerate at the four degeneration surfaces. The four such Killing vectors located at the roots $\eta_{ \pm}$and $\xi_{ \pm}$are

$$
k_{\eta_{ \pm}}=\partial_{z}-\frac{2}{\mathcal{G}_{ \pm}^{\prime}}\left(\partial_{\psi}-\eta_{ \pm} \partial_{\phi}\right), \quad l_{\xi_{ \pm}}=\partial_{z}+\frac{2}{\mathcal{F}_{ \pm}^{\prime}}\left(\partial_{\psi}-\xi_{ \pm} \partial_{\phi}\right)
$$

and correspond to translation generators with period $2 \pi$. Here and in the following we denote the evaluation of the functions $\mathcal{F}, \mathcal{G}$ at their respective roots with a \pm subscript, e.g. $\mathcal{F}_{ \pm}^{\prime} \equiv \mathcal{F}^{\prime}\left(\xi_{ \pm}\right)$. Clearly as we have four Killing vectors in a three-dimensional vector space there is a linear relation between them

$$
c k_{\eta_{-}}+d k_{\eta_{+}}-a l_{\xi_{-}}-b l_{\xi_{+}}=0
$$

for relatively prime integers $a, b, c, d$. To avoid conical singularities on the intersection of two degeneration surfaces we must demand that $a, b$ are each coprime to

\footnotetext{
${ }^{11}$ It may be possible to obtain a regular solution if the two ranges overlap at an endpoint. This however, will lead to a manifold with different topology to those where this is not the case. We shall therefore ignore this possibility and take $\eta \neq \xi$ everywhere.
} 
each of $c, d$. The linear relation implies the three conditions

$$
\begin{aligned}
a+b-c-d & =0, \\
\frac{a}{\mathcal{F}_{-}^{\prime}}+\frac{b}{\mathcal{F}_{+}^{\prime}}+\frac{c}{\mathcal{G}_{-}^{\prime}}+\frac{d}{\mathcal{G}_{+}^{\prime}} & =0, \\
\frac{a \xi_{-}}{\mathcal{F}_{-}^{\prime}}+\frac{b \xi_{+}}{\mathcal{F}_{+}^{\prime}}+\frac{c \eta_{-}}{\mathcal{G}_{-}^{\prime}}+\frac{d \eta_{+}}{\mathcal{G}_{+}^{\prime}} & =0 .
\end{aligned}
$$

The Killing vectors $\partial_{z}, \partial_{\psi}, \partial_{\phi}$ do not generate an effective torus action. Such a basis, $\partial_{\psi_{i}}$ with $2 \pi$ period, is given by

$$
\left(\begin{array}{l}
k_{\eta_{+}} \\
k_{\eta_{-}} \\
l_{\xi_{+}} \\
l_{\xi_{-}}
\end{array}\right)=\left(\begin{array}{ccc}
1 & 0 & 0 \\
1 & a k & b \\
1 & -a l & c \\
1 & 1 & 0
\end{array}\right)\left(\begin{array}{l}
\partial_{\psi_{1}} \\
\partial_{\psi_{2}} \\
\partial_{\psi_{3}}
\end{array}\right) .
$$

Here, by using Bézout's identity, we have introduced the integers $k, l$ satisfying

$$
b l+c k=1 .
$$

The manifolds $Y_{5}$ are characterized by the three integers $a, b, c$. The toric data of the family of manifolds $Y_{5}$ studied in this section, which can be read off from 6.83), coincides with the toric data of the $L^{a, b, c}$ Sasaki-Einstein metrics. Therefore we will, with slight abuse of notation, also refer in the following to the manifolds fibered over the Riemann surfaces as $Y_{5}=L^{a, b, c}$. Of course, at the moment we have not been able to impose any inequalities on the integers $a, b, c, d$ let alone state that they are positive, this can only be imposed with an explicit solution.

\subsubsection{Flux quantization}

In order for the class of supersymmetric solutions of type IIB supergravity to lead to well-defined string theory backgrounds we need to impose flux quantization. The only non-vanishing flux in the class of solutions we consider is the self-dual five-form flux 6.24). Therefore we need to impose flux quantization over all five-cycles in the compact geometry $Y_{7}$. There are two classes of five-cycles to consider. The first is given by the fiber $Y_{5}$ for a fixed point on the Riemann surface. Since we consider string theory setups with D3-branes wrapped over a Riemann surface $\Sigma_{g}$ and probing a Calabi-Yau singularity, we can identify the number of flux quanta through $Y_{5}$ with the total number of D3-branes $N$. The second class consists of five-cycles $\Sigma_{a}$ that are given by three-cycles $S_{a} \subset Y_{5}$ fibered over the Riemann 
surface. In going to one of the degeneration surfaces considered above one necessarily finds the three-cycle $S_{a}$. In order to impose flux quantization on the supergravity background we need to compute integrals of the five-form over these five-cycles. Once all supersymmetry conditions, regularity conditions and equations of motion are solved, this is of course a straightforward task. However, as stated before, we keep the discussion here as general as possible and will not assume any particular form of the functions $\mathcal{F}(\xi), \mathcal{G}(\eta)$ and $\mathcal{H}(\eta, \xi)$ solving the master equation 6.26. Remarkably, all the integrals can be performed without detailed knowledge of these functions. To perform the integrals we will only assume that the functions are solutions to the constraints 6.136 and $\mathcal{L}=\eta \xi$.

Integration over $\boldsymbol{Y}_{\mathbf{5}}$. We start by integrating the five-form field strength over the five-cycle $Y_{5}$. The flux $(6.27)$ can be easily inferred from the general expression 6.24 for supersymmetric solutions. Defining

$$
N=\frac{1}{\left(2 \pi \ell_{s}\right)^{4} g_{s}} \int_{Y_{5}} F_{5}
$$

one then finds

$$
\begin{aligned}
& N=\frac{L^{4}}{\left(2 \pi \ell_{s}\right)^{4} g_{s}} \int_{Y_{5}} \mathrm{~d}(z, \eta, \xi, \phi, \psi) \frac{1}{8 \mathcal{H}^{2}}\left[\mathcal{H}^{2}\left(\mathcal{F}^{\prime \prime}+\mathcal{G}^{\prime \prime}\right)-\mathcal{F}\left(\partial_{\xi} \mathcal{H}\right)^{2}\right. \\
&\left.-\mathcal{G}\left(\partial_{\eta} \mathcal{H}\right)^{2}+\mathcal{H}\left(\mathcal{F}^{\prime} \partial_{\xi} \mathcal{H}+\mathcal{F} \partial_{\xi}^{2} \mathcal{H}+\mathcal{G}^{\prime} \partial_{\eta} \mathcal{H}+\mathcal{G} \partial_{\eta}^{2} \mathcal{H}\right)\right],
\end{aligned}
$$

where we used the shorthand notation $\mathrm{d}\left(\omega_{1}, \ldots, \omega_{n}\right)=\bigwedge_{i=1}^{n} \mathrm{~d} \omega_{i}$. The integrand in 6.86 can be rewritten as a total derivative

$$
\begin{aligned}
N=\frac{L^{4}}{\left(2 \pi \ell_{s}\right)^{4} g_{s}} \int_{Y_{5}} \mathrm{~d}(z, \eta, \xi, \phi, \psi) \frac{1}{8}\left[\partial_{\xi} \partial_{\eta}\left(\eta \mathcal{F}^{\prime}+\xi \mathcal{G}^{\prime}\right)\right. & \\
& \left.+\partial_{\xi}\left(\mathcal{F} \frac{\partial_{\xi} \mathcal{H}}{\mathcal{H}}\right)+\partial_{\eta}\left(\mathcal{G} \frac{\partial_{\eta} \mathcal{H}}{\mathcal{H}}\right)\right]
\end{aligned}
$$

which we can now integrate explicitly. The last last two terms in 6.87 vanish upon performing the $\eta$ and $\xi$ integration respectively, since $\mathcal{F}\left(\xi_{ \pm}\right)=\mathcal{G}\left(\eta_{ \pm}\right)=0$. For the first term in 6.87 the $\eta, \xi$ integrations are now trivial. To perform the angular integration we use three coordinates generating an effective action for the three-torus. These follow from the relation 6.83 which implies the coordinate 
transformation

$$
\begin{aligned}
& z=\psi_{1} \\
& \psi=-\frac{2}{\mathcal{G}_{+}^{\prime}} \psi_{1}+2\left(\frac{1}{\mathcal{F}_{-}^{\prime}}+\frac{1}{\mathcal{G}_{+}^{\prime}}\right) \psi_{2}+2\left(\frac{k}{\mathcal{F}_{+}^{\prime}}-\frac{l}{\mathcal{G}_{-}^{\prime}}+\frac{l+k}{\mathcal{G}_{+}^{\prime}}\right) \psi_{3}, \\
& \phi=\frac{2 \eta_{+}}{\mathcal{G}_{+}^{\prime}} \psi_{1}-2\left(\frac{\xi_{-}}{\mathcal{F}_{-}^{\prime}}+\frac{\eta_{+}}{\mathcal{G}_{+}^{\prime}}\right) \psi_{2}-2\left(\frac{k \xi_{+}}{\mathcal{F}_{+}^{\prime}}-\frac{l \eta_{-}}{\mathcal{G}_{-}^{\prime}}+\frac{(l+k) \eta_{+}}{\mathcal{G}_{+}^{\prime}}\right) \psi_{3},
\end{aligned}
$$

with $\psi_{i}$ all having period $2 \pi$. The angular part is then equal to

$$
\int \mathrm{d}(z, \phi, \psi)=A_{J} \int \mathrm{d}\left(\psi_{1}, \psi_{2}, \psi_{3}\right)=(2 \pi)^{3} A_{J},
$$

where, using the regularity conditions 6.82 and the relation 6.84 for $l$, we can write

$$
\begin{aligned}
A_{J}= & \frac{4}{(a+b-c) c\left(\eta_{+}-\eta_{-}\right)\left(\mathcal{F}_{-}^{\prime} \mathcal{F}_{+}^{\prime}\right)^{2}}\left(b\left(\eta_{-}-\xi_{+}\right)\left(\xi_{+}-\eta_{+}\right)\left(\mathcal{F}_{-}^{\prime}\right)^{2}\right. \\
& +\left[-a\left(\eta_{+}-\xi_{-}\right)\left(\eta_{-}-\xi_{+}\right)+b\left(\eta_{-}-\xi_{-}\right)\left(\eta_{+}-\xi_{+}\right)\right. \\
& \left.\left.\quad-c\left(\eta_{-}-\eta_{+}\right)\left(\xi_{-}-\xi_{+}\right)\right] \mathcal{F}_{-}^{\prime} \mathcal{F}_{+}^{\prime}-a\left(\eta_{-}-\xi_{-}\right)\left(\xi_{-}-\eta_{+}\right)\left(\mathcal{F}_{+}^{\prime}\right)^{2}\right) .
\end{aligned}
$$

Combining the angular part with the part coming from the integration over $\xi$ and $\eta$ results in

$$
N=\frac{L^{4}}{16 \pi g_{s} \ell_{s}^{4}} A_{J}\left(\left(\eta_{-}-\eta_{+}\right)\left(\mathcal{F}_{-}^{\prime}-\mathcal{F}_{+}^{\prime}\right)+\left(\xi_{-}-\xi_{+}\right)\left(\mathcal{G}_{-}-\mathcal{G}_{+}\right)\right) .
$$

Note that 6.91 is independent of the function $\mathcal{H}$ and is therefore independent of the flavour twisting.

Integration over $\boldsymbol{\Sigma}_{\boldsymbol{a}}$. We now turn to the five-cycles $\Sigma_{a}$ which are fibrations of three-cycles $S_{a} \subset Y_{5}$ over the Riemann surface. These three-cycles are the degeneration surfaces of the Killing vectors 6.80 and we denote them by

$$
\left(S_{\xi_{+}}, S_{\eta_{+}}, S_{\xi_{-}}, S_{\eta_{-}}\right)=\left(S_{1}, S_{2}, S_{3}, S_{4}\right)
$$

Thus we have four additional five-cycles $\Sigma_{a} \in H_{5}\left(Y_{7}, \mathbb{Z}\right)$. However, as can be seen from 6.45, these five-cycles are in fact not independent. Due to these relations there is only one independent flux quantum number in this class. Nevertheless, we proceed in the following by performing the integrals of the five-form field strength over all five-cycles $\Sigma_{\xi_{ \pm}}$and $\Sigma_{\eta_{ \pm}}$. 
We first perform the integral over $\Sigma_{\eta_{+}}$, for which we have to integrate over $\xi$, the Riemann surface and two angular coordinates. From 6.83 we see that the coordinate $\psi_{1}$ in 6.88 corresponds to the degenerating Killing vector $k_{\eta_{+}}$. Therefore, $\psi_{2}$ and $\psi_{3}$ will give an effective action of the two-torus that is obtained by contracting the one-cycle of the degenerating Killing vector of the toric $\mathrm{T}^{3}$. It turns out that the integral of the field strength can be written as

$$
\begin{aligned}
M_{\eta_{+}}=\frac{L^{4}}{\left(2 \pi \ell_{s}\right)^{4} g_{s}} \frac{\mathcal{G}_{+}^{\prime} A_{J}}{16} \int_{\Sigma_{\eta_{+}}} J_{g} \wedge \mathrm{d}\left(\xi, \psi_{2}, \psi_{3}\right)\left[-\partial_{\xi}\left(\mathcal{H}\left(\eta_{+}, \xi\right) \frac{\mathcal{F}^{\prime}+\mathcal{G}_{+}^{\prime}}{\eta_{+}-\xi}\right)\right. \\
+\partial_{\xi}(2 \kappa \xi)-\partial_{\xi}\left(\partial_{\xi} \mathcal{H}\left(\eta_{+}, \xi\right) \frac{\mathcal{F}}{\eta_{+}-\xi}\right) \\
\left.\quad-\frac{2 \mathcal{F} \partial_{\xi}^{2} \mathcal{H}\left(\eta_{+}, \xi\right)-\mathcal{G}_{+}^{\prime}\left[\partial_{\xi} \mathcal{H}\left(\eta_{+}, \xi\right)-\partial_{\eta} \mathcal{H}\left(\eta_{+}, \xi\right)\right]}{\eta_{+}-\xi}\right] .
\end{aligned}
$$

The last piece in 6.93 seems to spoil the possibility to integrate this equation completely, as it is not manifestly written as a total derivative. We can now utilize the first equation in 6.136 and evaluate it at $\eta=\eta_{+}$from which we find that $\partial_{\xi}^{2} \mathcal{H}\left(\eta_{+}, \xi\right)=0$. The third equation of 6.136 evaluated at $\eta=\eta_{+}$allows us to trade the difference of the $\xi$ and $\eta$ derivatives with a total derivative $\partial_{\xi} \partial_{\eta} \mathcal{H}\left(\eta_{+}, \xi\right)$. We have therefore shown that, independent of the details of the functions in the metric ansatz, we can write the integrand once again as a total derivative and we can integrate it explicitly. We obtain

$$
\begin{aligned}
M_{\eta_{+}}=M_{2}= & \frac{L^{4}}{(2 \pi)^{2} g_{s} \ell_{s}^{4}} \frac{\mathcal{G}_{+}^{\prime} A_{J}}{16} \operatorname{vol}\left(\Sigma_{g}\right)\left(2 \kappa\left(\xi_{+}-\xi_{-}\right)+\frac{\left(\mathcal{F}_{-}^{\prime}+\mathcal{G}_{+}^{\prime}\right) \mathcal{H}\left(\eta_{+}, \xi_{-}\right)}{\eta_{+}-\xi_{-}}\right. \\
& \left.-\frac{\left(\mathcal{F}_{+}^{\prime}+\mathcal{G}_{+}^{\prime}\right) \mathcal{H}\left(\eta_{+}, \xi_{+}\right)}{\eta_{+}-\xi_{+}}+\mathcal{G}_{+}^{\prime}\left(\partial_{\eta} \mathcal{H}\left(\eta_{+}, \xi_{+}\right)-\partial_{\eta} \mathcal{H}\left(\eta_{+}, \xi_{-}\right)\right)\right) .
\end{aligned}
$$

Similarly we can integrate the five-form over the remaining three five-cycles $\Sigma_{\eta_{-}}$ and $\Sigma_{\xi_{ \pm}}$. Instead of giving the full details about the integration we only give the final results for the remaining fluxes. Note that for each integration one has to find a coordinate system as in 6.88 to perform the integration, such that $\partial_{\psi_{1}}$ is equal to the Killing vector that degenerates at the relevant degeneration surface. In appendix 6.B we provide the coordinate systems that we use for the different degeneration surfaces. The value of the flux $M_{\eta_{-}}$threading through the five-cycle 
defined by the degeneration surface $\eta=\eta_{-}$is given by

$$
\begin{aligned}
M_{\eta_{-}}=M_{4}= & -\frac{L^{4}}{(2 \pi)^{2} g_{s} \ell_{s}^{4}} \frac{\mathcal{G}_{-}^{\prime} A_{J}}{16} \operatorname{vol}\left(\Sigma_{g}\right)\left(2 \kappa\left(\xi_{+}-\xi_{-}\right)+\frac{\left(\mathcal{F}_{-}^{\prime}+\mathcal{G}_{-}^{\prime}\right) \mathcal{H}\left(\eta_{-}, \xi_{-}\right)}{\eta_{-}-\xi_{-}}\right. \\
& \left.-\frac{\left(\mathcal{F}_{+}^{\prime}+\mathcal{G}_{-}^{\prime}\right) \mathcal{H}\left(\eta_{-}, \xi_{+}\right)}{\eta_{-}-\xi_{+}}+\mathcal{G}_{-}^{\prime}\left(\partial_{\eta} \mathcal{H}\left(\eta_{-}, \xi_{+}\right)-\partial_{\eta} \mathcal{H}\left(\eta_{-}, \xi_{-}\right)\right)\right) \cdot
\end{aligned}
$$

The fluxes associated with the $\xi_{+}$and $\xi_{-}$degeneration surfaces are given by

$$
\begin{aligned}
M_{\xi_{+}}=M_{1} & =\frac{L^{4}}{(2 \pi)^{2} g_{s} \ell_{s}^{4}} \frac{\mathcal{F}_{+}^{\prime} A_{J}}{16} \operatorname{vol}\left(\Sigma_{g}\right)\left(2 \kappa\left(\eta_{+}-\eta_{-}\right)+\frac{\left(\mathcal{F}_{+}^{\prime}+\mathcal{G}_{-}^{\prime}\right) \mathcal{H}\left(\eta_{-}, \xi_{+}\right)}{\eta_{-}-\xi_{+}}\right. \\
& \left.-\frac{\left(\mathcal{F}_{+}^{\prime}+\mathcal{G}_{+}^{\prime}\right) \mathcal{H}\left(\eta_{+}, \xi_{+}\right)}{\eta_{+}-\xi_{+}}+\mathcal{F}_{+}^{\prime}\left(\partial_{\xi} \mathcal{H}\left(\eta_{-}, \xi_{+}\right)-\partial_{\xi} \mathcal{H}\left(\eta_{+}, \xi_{+}\right)\right)\right)
\end{aligned}
$$

and

$$
\begin{aligned}
M_{\xi_{-}}=M_{3}= & -\frac{L^{4}}{(2 \pi)^{2} g_{s} \ell_{s}^{4}} \frac{\mathcal{F}_{-}^{\prime} A_{J}}{16} \operatorname{vol}\left(\Sigma_{g}\right)\left(2 \kappa\left(\eta_{+}-\eta_{-}\right)+\frac{\left(\mathcal{F}_{-}^{\prime}+\mathcal{G}_{-}^{\prime}\right) \mathcal{H}\left(\eta_{-}, \xi_{-}\right)}{\eta_{-}-\xi_{-}}\right. \\
& \left.-\frac{\left(\mathcal{F}_{-}^{\prime}+\mathcal{G}_{+}^{\prime}\right) \mathcal{H}\left(\eta_{+}, \xi_{-}\right)}{\eta_{+}-\xi_{-}}+\mathcal{F}_{-}^{\prime}\left(\partial_{\xi} \mathcal{H}\left(\eta_{-}, \xi_{-}\right)-\partial_{\xi} \mathcal{H}\left(\eta_{+}, \xi_{-}\right)\right)\right)
\end{aligned}
$$

respectively.

\subsubsection{General formula for central charge and R-charges}

We now continue the general discussion of the $\mathrm{AdS}_{3}$ solutions of interest with the computation of the central charge and R-charges of the dual 2d SCFT. To leading order in the large $N$ limit the central charge can be computed by evaluating 6.34 for the solution. Again we assume no additional form of the functions in the metric ansatz. Using the explicit expression 6.74 for $J$, and the expression 6.77) for $P$ to compute $\eta_{R}$ and $\rho$, one obtains the central charge

$$
\begin{aligned}
c_{\text {sugra }}=\frac{3 L^{8}}{64 \pi^{6} g_{s}^{2} \ell_{s}^{8}} \frac{1}{4} \int_{Y_{7}} \mathrm{~d}(z, \eta, \xi, \phi, \psi) & \wedge J_{g}\left(\mathcal{H}(\eta, \xi)\left(\mathcal{F}^{\prime \prime}(\xi)+\mathcal{G}^{\prime \prime}(\eta)\right)\right. \\
+2 \mathcal{F}^{\prime}(\xi) \partial_{\xi} & \mathcal{H}(\eta, \xi)+2 \kappa(\xi-\eta)+3 \mathcal{F}(\xi) \partial_{\xi}^{2} \mathcal{H}(\eta, \xi) \\
+ & \left.2 \mathcal{G}^{\prime}(\eta) \partial_{\eta} \mathcal{H}(\eta, \xi)+3 \mathcal{G}(\eta) \partial_{\eta}^{2} \mathcal{H}(\eta, \xi)\right) .
\end{aligned}
$$

Remarkably, it is again possible to rewrite 6.98 as a total derivative,

$$
\begin{aligned}
c_{\text {sugra }}= & \frac{3 L^{8}}{64 \pi^{6} g_{s}^{2} \ell_{s}^{8}} \frac{1}{4} \int_{Y_{7}} \mathrm{~d}(z, \eta, \xi, \phi, \psi) \wedge J_{g} \partial_{\eta} \partial_{\xi}\left[\frac { 1 } { 2 } \left(\eta \mathcal{F}^{\prime}(\xi)\left(2 \mathcal{H}(\eta, \xi)-\eta \partial_{\eta} \mathcal{H}(\eta, \xi)\right)\right.\right. \\
& +\eta \mathcal{F}(\xi)\left(2 \partial_{\xi} \mathcal{H}(\eta, \xi)-\eta \partial_{\eta} \partial_{\xi} \mathcal{H}(\eta, \xi)\right)+\xi \mathcal{G}^{\prime}(\eta)\left(2 \mathcal{H}(\eta, \xi)-\xi \partial_{\xi} \mathcal{H}(\eta, \xi)\right) \\
& \left.\left.+\xi \mathcal{G}(\eta)\left(2 \partial_{\eta} \mathcal{H}(\eta, \xi)-\xi \partial_{\eta} \partial_{\xi} \mathcal{H}(\eta, \xi)\right)\right)+\kappa\left(\eta \xi^{2}-\eta^{2} \xi\right)\right],
\end{aligned}
$$


up to terms which vanish upon evaluation on the boundaries of the integration over $\xi$ and $\eta$. Integrating the latter equation is now straightforward and leads to the lengthy but fully general expression for the central charge

$$
\begin{aligned}
c_{\text {sugra }}= & \frac{3 L^{8}}{(2 \pi)^{3} g_{s}^{2} \ell_{s}^{8}} \frac{A_{J}}{8} \operatorname{vol}\left(\Sigma_{g}\right)\left(2\left(\eta_{-} \mathcal{F}_{-}^{\prime}+\xi_{-} \mathcal{G}_{-}^{\prime}\right) \mathcal{H}\left(\eta_{-}, \xi_{-}\right)\right. \\
& -2\left(\eta_{-} \mathcal{F}_{+}^{\prime}+\xi_{+} \mathcal{G}_{-}^{\prime}\right) \mathcal{H}\left(\eta_{-}, \xi_{+}\right)-2\left(\eta_{+} \mathcal{F}_{-}^{\prime}+\xi_{-} \mathcal{G}_{+}^{\prime}\right) \mathcal{H}\left(\eta_{+}, \xi_{-}\right) \\
& +2\left(\eta_{+} \mathcal{F}_{+}^{\prime}+\xi_{+} \mathcal{G}_{+}^{\prime}\right) \mathcal{H}\left(\eta_{+}, \xi_{+}\right)-\eta_{-}^{2} \mathcal{F}_{-}^{\prime} \partial_{\eta} \mathcal{H}\left(\eta_{-}, \xi_{-}\right) \\
& +\eta_{-}^{2} \mathcal{F}_{+}^{\prime} \partial_{\eta} \mathcal{H}\left(\eta_{-}, \xi_{+}\right)+\eta_{+}^{2} \mathcal{F}_{-}^{\prime} \partial_{\eta} \mathcal{H}\left(\eta_{+}, \xi_{-}\right)-\eta_{+}^{2} \mathcal{F}_{+}^{\prime} \partial_{\eta} \mathcal{H}\left(\eta_{+}, \xi_{+}\right) \\
& -\xi_{-}^{2} \mathcal{G}_{-}^{\prime} \partial_{\xi} \mathcal{H}\left(\eta_{-}, \xi_{-}\right)+\xi_{+}^{2} \mathcal{G}_{-}^{\prime} \partial_{\xi} \mathcal{H}\left(\eta_{-}, \xi_{+}\right)+\xi_{-}^{2} \mathcal{G}_{+}^{\prime} \partial_{\xi} \mathcal{H}\left(\eta_{+}, \xi_{-}\right) \\
& \left.-\xi_{+}^{2} \mathcal{G}_{+}^{\prime} \partial_{\xi} \mathcal{H}\left(\eta_{+}, \xi_{+}\right)-2 \kappa\left(\eta_{-}-\eta_{+}\right)\left(\xi_{-}-\xi_{+}\right)\left(\eta_{-}+\eta_{+}-\xi_{-}-\xi_{+}\right)\right) .
\end{aligned}
$$

The central charge of the dual $2 \mathrm{~d}$ SCFT is thus expressed in terms of the functions $\mathcal{F}, \mathcal{G}, \mathcal{H}$ and their derivatives evaluated at the roots. Let us now compute the R-charges of the baryonic operators which are given by the integrals 6.47 evaluated on the three-cycles $S_{\xi_{ \pm}}$and $S_{\eta_{ \pm}}$. For these integrals we again use the coordinate system relevant for the particular degeneration surface and that the Kähler form of the transverse space may be written as

$$
\omega=\mathrm{d} \xi \wedge(\mathrm{d} \phi+\eta \mathrm{d} \psi)+\mathrm{d} \eta \wedge(\mathrm{d} \phi+\xi \mathrm{d} \psi) .
$$

As opposed to the flux integrals and the central charge, where rewriting the integrands as total derivatives was non-trivial, the integrals for the R-charges are considerably easier to perform. For all four three-cycles $S_{a}$ the corresponding integrands are simply constant. The R-charges are given by:

$$
\begin{aligned}
& R_{\eta_{+}}=R_{2}=-\frac{L^{4}\left(\xi_{+}-\xi_{-}\right)\left[\left(\eta_{+}-\eta_{-}\right) \mathcal{F}_{+}^{\prime}+\left(\eta_{+}-\xi_{+}\right) \mathcal{G}_{-}^{\prime}+\left(\xi_{+}-\eta_{-}\right) \mathcal{G}_{+}^{\prime}\right]}{2 \pi a g_{s} \ell_{s}^{4} \mathcal{F}_{+}^{\prime} \mathcal{G}_{-}^{\prime}}, \\
& R_{\eta_{-}}=R_{4}=\frac{L^{4}\left(\xi_{+}-\xi_{-}\right)\left[\left(\eta_{+}-\eta_{-}\right) \mathcal{F}_{+}^{\prime}+\left(\eta_{+}-\xi_{+}\right) \mathcal{G}_{-}^{\prime}+\left(\xi_{+}-\eta_{-}\right) \mathcal{G}_{+}^{\prime}\right]}{2 \pi a g_{s} \ell_{s}^{4} \mathcal{F}_{+}^{\prime} \mathcal{G}_{+}^{\prime}} \\
& R_{\xi_{+}}=R_{1}=-\frac{L^{4}\left(\eta_{+}-\eta_{-}\right)\left[\left(\eta_{+}-\eta_{-}\right) \mathcal{F}_{+}^{\prime}+\left(\eta_{+}-\xi_{+}\right) \mathcal{G}_{-}^{\prime}+\left(\xi_{+}-\eta_{-}\right) \mathcal{G}_{+}^{\prime}\right]}{2 \pi a g_{s} \ell_{s}^{4} \mathcal{G}_{-}^{\prime} \mathcal{G}_{+}^{\prime}} \\
& R_{\xi_{-}}=R_{3}=\frac{L^{4} \mathcal{F}_{-}^{\prime}\left(\eta_{+}-\eta_{-}\right)\left[\left(\eta_{+}-\eta_{-}\right) \mathcal{F}_{+}^{\prime}+\left(\eta_{+}-\xi_{+}\right) \mathcal{G}_{-}^{\prime}+\left(\xi_{+}-\eta_{-}\right) \mathcal{G}_{+}^{\prime}\right]}{2 \pi a g_{s} \ell_{s}^{4} \mathcal{F}_{+}^{\prime} \mathcal{G}_{-}^{\prime} \mathcal{G}_{+}^{\prime}} .
\end{aligned}
$$


For writing the R-charges in this form we used the regularity conditions 6.82). If we solve 6.91 for the string coupling it is not hard to show that the first component of the sum $\sqrt{6.49}$ is indeed satisfied ${ }^{12}$ From the remaining two components of 6.49 we can determine the (off-shell) R-symmetry vector $\vec{b}$.

\subsubsection{Solving the master equation}

In this section we solve the master equation 6.26 in order to determine explicit $\mathrm{AdS}_{3}$ solutions. We will use ansätze for the functions $\mathcal{L}, \mathcal{H}, \mathcal{F}$ and $\mathcal{G}$. As previously stated we take $\mathcal{L}=\eta \xi$ in order to recover the universal twist solution 223 and this fixes $\mathcal{H}$ to be

$$
\mathcal{H}=\alpha_{0}+\alpha_{1}(\eta+\xi)+\alpha_{2} \eta \xi
$$

However, some of the parameters are redundant and can be removed by using the symmetries 6.72 of the metric 6.70. In writing the simplest form of $\mathcal{H}$ we have to distinguish between different cases. Firstly, when $\alpha_{2} \neq 0$ we use the symmetry 6.72 to set $\alpha_{1}=0$ and $\alpha_{2}=1$ by taking

$$
\alpha=\frac{1}{\sqrt{\left|\alpha_{2}\right|}}, \quad \beta=-\frac{\alpha_{1}}{\alpha_{2}}, \quad \gamma=1 .
$$

Therefore, we can take

$$
\mathcal{H}=\alpha_{0}+\eta \xi
$$

When $\alpha_{2}=0$ and $\alpha_{1} \neq 0$ we can use similar transformations to write $\mathcal{H}$ in the form

$$
\mathcal{H}=\eta+\xi
$$

The last case is when $\alpha_{1}=\alpha_{2}=0$, for which we have

$$
\mathcal{H}=\alpha_{0}
$$

Note that the metric 6.70 in this last case reduces to a direct product of the Riemann surface and the orthotoric metric $\mathrm{d} s^{2}\left(\mathcal{M}_{4}\right)$,

$$
\mathrm{d} s^{2}=\mathrm{d} s^{2}\left(\Sigma_{g}\right)+\mathrm{d} s^{2}\left(\mathcal{M}_{4}\right)
$$

which corresponds to the case of no twisting with the flavour symmetries. Since this case has been considered in the literature for $\mathrm{T}^{2}$ in 204 and $\Sigma_{g>1}$ in 223 . (the $\mathrm{S}^{2}$ case does not exist), we will not consider it further in this chapter.

\footnotetext{
${ }^{12}$ Recall that $b_{1}=2$ is fixed.
} 
For the functions $\mathcal{F}$ and $\mathcal{G}$ we take the ansätze

$$
\mathcal{F}(\xi)=\sum_{i=-5}^{5} \tilde{f}_{i} \xi^{i}, \quad \mathcal{G}(\eta)=\sum_{i=-5}^{5} \tilde{g}_{i} \eta^{i}
$$

Using the expression for $\mathcal{H}$ above, we solve the master equation to determine the coefficients $\tilde{f}_{i}, \tilde{g}_{i}$ and the remaining coefficients in $\mathcal{H}$. The case 6.106 with $\alpha_{2}=0$ turns out to never give a sensible globally defined compact solution and therefore we restrict ourselves solely to the case $\alpha_{2} \neq 0$ for which $\mathcal{H}$ is given by 6.105. To solve the master equation we use the expression 6.71 for $\mathcal{A}_{g}$ and the metric $\mathrm{d} s^{2}\left(\Sigma_{g}\right)$ to treat all Riemann surfaces universally. Solving the master equation with $\mathcal{H}$ given by 6.105 and our polynomial ansätze for $\mathcal{F}$ and $\mathcal{G}$ implies $\alpha_{0}=0$ and after a suitable redefinition of parameters the functions $\mathcal{F}$ and $\mathcal{G}$ are given by

$$
\mathcal{F}(\xi)=\frac{-A(\xi+C)^{2}+\kappa \xi^{2}+D \xi^{3}}{\xi}, \quad \mathcal{G}(\eta)=\frac{-B(\eta+C)^{2}-\kappa \eta^{2}-D \eta^{3}}{\eta}
$$

where $\kappa=1,0,-1$ for $\Sigma_{g}=\mathrm{S}^{2}, \mathrm{~T}^{2}, \Sigma_{g>1}$ respectively. The functions $\mathcal{F}, \mathcal{G}$ and $\mathcal{H}$ are thus determined by four parameters $A, B, C$ and $D{ }^{13}$ Recall that we have not used up all of the gauge symmetry and we may use the remainder to set $D= \pm 1$. These functions need to satisfy the regularity conditions 6.82 which allows us to solve for two of the remaining three parameters in terms of the other one and $a$, $b$ and $c$. The final solution thus depends on four parameters. Consider now the central charge 6.100). The coefficient $L^{8} /\left(g_{s}^{2} \ell_{s}^{8}\right)$ can be expressed in terms of $N^{2}$ using 6.91. The central charge, and also the R-charges 6.102, then depend on five parameters instead of the seven parameters $a, b, c, m_{1}, N, n_{2}$ and $n_{3}$ that we find on the c-extremization side. Ergo, we have not found the most general class of solutions. We have motivated that the ansatz for $\mathcal{H}$ is the most general that we could take, which implies that the ansätze for $\mathcal{F}$ and $\mathcal{G}$ should be modified to account for the extra parameters. Note that the functions 6.110 have three roots which need to be real. Their domains, given by $\left[\xi_{-}, \xi_{+}\right]$and $\left[\eta_{-}, \eta_{+}\right]$respectively, should be such that $0<\xi_{-}<\xi_{+}<\eta_{-}<\eta_{+}$, as assumed without loss of generality in the regularity analysis. Lastly, the functions should be positive on their respective domains given the above choice. These conditions translate into restrictions on $A$, $B$, and $C$, which we examine in more detail in the next subsection.

\footnotetext{
${ }^{13}$ Note that here the coefficients $A$ and $B$ do not correspond to the variables in the previous sections where they correspond to respectively the Kähler class of the Riemann surface and the baryonic flux.
} 


\subsubsection{Solving the regularity equations and matching to the field theory}

So far we have derived the local form of solutions satisfying 6.26, in this section we extend these local solutions to globally well-defined ones by imposing the regularity conditions 6.82. With the global solutions in hand we can compute the central charges and R-charges of the solutions and compare to the field theory results, finding perfect agreement. We perform this matching numerically for a large set of solutions, a few of which we present for exposition.

To solve the regularity conditions we use that the functions $\mathcal{F}$ and $\mathcal{G} 6.110$ can be rewritten in terms of their roots as

$$
\begin{aligned}
& \mathcal{F}(\xi)=\frac{D\left(\xi-\xi_{-}\right)\left(\xi-\xi_{+}\right)\left(\xi-\xi_{*}\right)}{\xi}, \\
& \mathcal{G}(\eta)=-\frac{D\left(\eta-\eta_{-}\right)\left(\eta-\eta_{+}\right)\left(\eta-\eta_{*}\right)}{\eta}
\end{aligned}
$$

Here we have denoted the third root of $\mathcal{F}(\xi)$ and $\mathcal{G}(\eta)$ by $\xi_{*}$ and $\eta_{*}$ respectively. The derivatives of the functions evaluated at $\xi_{ \pm}, \eta_{ \pm}$are given by

$$
\begin{array}{ll}
\mathcal{F}_{-}^{\prime}=\frac{D\left(\xi_{-}-\xi_{+}\right)\left(\xi_{-}-\xi_{*}\right)}{\xi_{-}}, & \mathcal{G}_{-}^{\prime}=-\frac{D\left(\eta_{-}-\eta_{+}\right)\left(\eta_{-}-\eta_{*}\right)}{\eta_{-}}, \\
\mathcal{F}_{+}^{\prime}=\frac{D\left(\xi_{+}-\xi_{-}\right)\left(\xi_{+}-\xi_{*}\right)}{\xi_{+}}, & \mathcal{G}_{+}^{\prime}=-\frac{D\left(\eta_{+}-\eta_{-}\right)\left(\eta_{+}-\eta_{*}\right)}{\eta_{+}} .
\end{array}
$$

With these expressions the fluxes 6.94 6.97, central charge 6.100 and Rcharges 6.102 can be expressed in terms of the six roots and $D^{14}$ However, these parameters are not independent. Equating 6.110 and 6.111 results in the conditions

$$
\begin{aligned}
& \xi_{-} \xi_{+} \xi_{*}=\frac{A C^{2}}{D}, \quad \xi_{-} \xi_{+}+\xi_{-} \xi_{*}+\xi_{+} \xi_{*}=-\frac{2 A C}{D}, \quad \xi_{-}+\xi_{+}+\xi_{*}=\frac{A-\kappa}{D}, \\
& \eta_{-} \eta_{+} \eta_{*}=-\frac{B C^{2}}{D}, \quad \eta_{-} \eta_{+}+\eta_{-} \eta_{*}+\eta_{+} \eta_{*}=\frac{2 B C}{D}, \quad \eta_{-}+\eta_{+}+\eta_{*}=-\frac{B+\kappa}{D} .
\end{aligned}
$$

Furthermore, the substitution of 6.112 into the regularity conditions 6.82 results

\footnotetext{
${ }^{14}$ Recall, this may be normalized to \pm 1 .
} 
in two further conditions

$$
\begin{array}{r}
a \xi_{-}\left(\xi_{+}-\xi_{*}\right)\left(\xi_{-}-\eta_{-}\right)\left(\eta_{*}-\eta_{+}\right)=\left(\xi_{-}-\xi_{*}\right)\left[b \xi_{+}\left(\xi_{+}-\eta_{-}\right)\left(\eta_{*}-\eta_{+}\right)\right. \\
\left.-d \eta_{+}\left(\xi_{-}-\xi_{+}\right)\left(\xi_{+}-\xi_{*}\right)\right], \\
a \xi_{-}\left(\xi_{+}-\xi_{*}\right)\left(\xi_{-}-\eta_{+}\right)\left(\eta_{*}-\eta_{-}\right)=\left(\xi_{-}-\xi_{*}\right)\left[b \xi_{+}\left(\xi_{+}-\eta_{+}\right)\left(\eta_{*}-\eta_{-}\right)\right. \\
\left.-c \eta_{-}\left(\xi_{-}-\xi_{+}\right)\left(\xi_{+}-\xi_{*}\right)\right] .
\end{array}
$$

We thus have eight equations for ten parameters which we will solve for $A, B$ and the roots. We first study $\kappa=0$ since it is the easiest case before turning our attention to the $\kappa \neq 0$ cases.

\subsubsection{Torus fibrations}

There are two choices that one can make, i.e. one can take $D= \pm 1$. It transpires that the $D=-1$ solution is incompatible with our choice of $0<\xi<\eta$ and therefore we take $D=1$ throughout this section. Since $D=1$ we have that the auxiliary root $\xi_{*}$ satisfies $\xi_{*}>\xi_{+}>\xi_{-}>0$ and therefore 6.113 implies firstly that $A>0$ and secondly that $C<0$. Moreover the positivity of the discriminant of $\xi \mathcal{F}(\xi)$ and $\eta \mathcal{G}(\eta)$ implies

$$
A>-\frac{27}{4} C>0, \quad B<\frac{27}{4} C<0 .
$$

It then follows from 6.113 that $\eta_{*}>0$. To aid the reader we have plotted a representative example of the functions $\mathcal{F}$ and $\mathcal{G}$ and their roots in figure 6.1

It is convenient to perform the following redefinition of the auxiliary roots:

$$
\eta_{*}=-\frac{C}{4}\left(v^{2}+3\right), \quad \xi_{*}=-\frac{3 C}{4}\left(\frac{3}{u^{2}}+1\right) .
$$

After this change of coordinates one can solve $(6.113)$ and find that the physical roots are located at

$$
\xi_{ \pm}=-\frac{3 C\left(3+u^{2}\right)}{(u \mp 3)^{2}}, \quad \eta_{ \pm}=-\frac{C\left(3+v^{2}\right)}{(v \mp 1)^{2}}
$$

whilst the constants $A$ and $B$ take the form

$$
A=-\frac{27 C\left(3+u^{2}\right)^{3}}{4 u^{2}\left(u^{2}-9\right)^{2}}, \quad B=\frac{C\left(3+v^{2}\right)^{3}}{4\left(v^{2}-1\right)^{2}} .
$$

The domains of $u$ and $v$ are fixed by imposing $0<\xi_{-}<\xi_{+}<\eta_{-}<\eta_{+}$and $\xi_{+}<\xi_{*}$, giving

$$
0<v<1, \quad 0<u<\frac{3(1-v)}{v+3}
$$




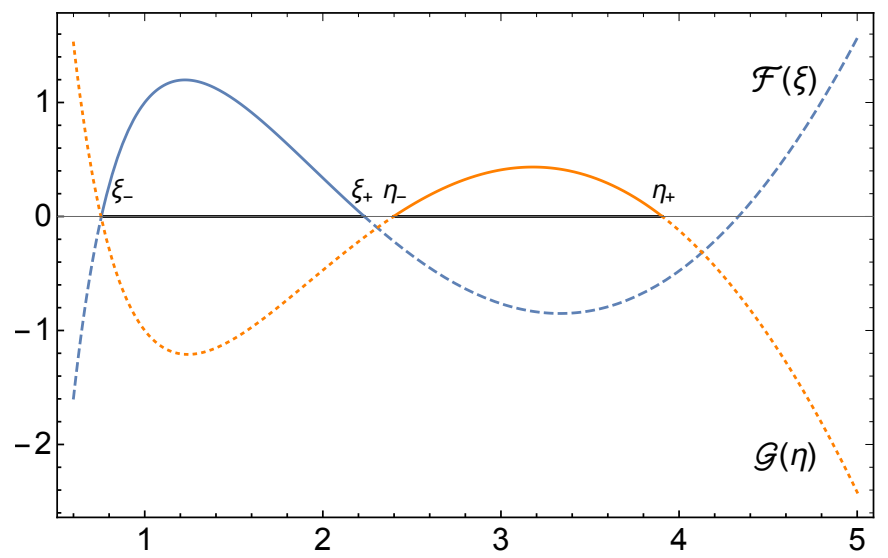

Figure 6.1: Plot of $\mathcal{F}(\xi)$ and $\mathcal{G}(\eta)$ for $a=-1, b=19, c=16, C=-1$ and $D=1$. The domain of $\mathcal{F}$ is given by $\left[\xi_{-}, \xi_{+}\right]$and the domain of $\mathcal{G}$ by $\left[\eta_{-}, \eta_{+}\right]$, this is drawn using the solid line. Outside of their domains $\mathcal{F}$ is given by the dashed blue line and $\mathcal{G}$ by the dotted orange line.

The only constraint on the constant $C$ is that it must be strictly negative, i.e. $C<0$. One can also see that these conditions are consistent with the Ricci scalar being strictly positive definite in the domain as is required by supersymmetry.

One should now solve the regularity conditions to obtain expressions for $u$ and $v$ in terms of the integers $a, b, c$. Expressed in terms of $u$ and $v$, the conditions are given by

$$
\begin{aligned}
& a=-b \frac{(1+u)\left((u+3)^{2} v^{2}-9(1-u)^{2}\right)}{(1-u)\left((u-3)^{2} v^{2}-9(1+u)^{2}\right)}, \\
& c=b \frac{u(3+v)(u(v-3)+3(1+v))}{3(1-u)(3(1-v)+u(3+v))},
\end{aligned}
$$

where we have also solved them for the integers $a$ and $c$. The solution of these equations that expresses $u$ and $v$ as function of $a, b$ and $c$ is a bit unwieldy. The variable $u$ is a root of the quintic

$$
\begin{aligned}
Z[X]= & -b c(b-c)(X-1)\left(3+4 X+X^{2}\right)^{2} \\
& +a^{2}(1-X)^{2}\left(8 b X^{2}+c(3-X)^{2}(1+X)\right)+a(1+X)\left[8 b^{2} X^{2}(1+X)\right. \\
& \left.-c^{2}\left(3-4 X+X^{2}\right)^{2}-2 b c\left(-9+9 X-7 X^{2}+7 X^{3}\right)\right] .
\end{aligned}
$$


Given such a root fixes $v$ to be

$$
\begin{aligned}
v= & -\frac{1}{12 a b(a+b)(a+b-2 c)}\left[12 a b(a+b)^{2}+3 c(a+b-c)\left(13 a^{2}-54 a b+13 b^{2}\right)\right. \\
& +\left(a^{2}-b^{2}\right)\left(16 a b-25(a+b) c+25 c^{2}\right) u \\
& +\left(5 a^{2}-22 a b+5 b^{2}\right)\left(8 a b-7(a+b) c+7 c^{2}\right) u^{2} \\
& \left.-\left(a^{2}-b^{2}\right)\left(32 a b-25(a+b) c+25 c^{2}\right) u^{3}-4(a-b)^{2}(a+b-c) c u^{4}\right] .
\end{aligned}
$$

One has to take the root $u$ such that $u, v$ are in the domain 6.119.

The expressions 6.120 can be used to determine the possible ranges of $a, b, c$ and $d$ compatible with globally well-defined solutions. Using the domain 6.119 of $u$ and $v$ we find that regular solutions always have

$$
a<0<d \leq c \leq b, \quad \operatorname{gcd}(a, c)=\operatorname{gcd}(a, d)=\operatorname{gcd}(b, c)=\operatorname{gcd}(b, d)=1,
$$

where we have taken $b>0$ without loss of generality. In particular we see that $a<0$ whilst the other three integers are positive. Note that from the naive comparison with the Sasaki-Einstein $L^{a, b, c}$ metric one would have taken $a>0$ and not $a<0$. This phenomenon has been noted in the literature previously for the Baryonic twist solutions of $L^{a, b, c}$ on the torus [204], here we have proven that it also extends to the twist solutions presented here.

We now turn to matching these solutions to the field theory side. We have done this numerically for explicit triples $a, b, c$ satisfying 6.123 15. More concretely we have matched the central charge $c_{\text {sugra }}$ and R-charges computed using the supergravity solution, i.e. by evaluating 6.100 and 6.102, to the central charge $c_{R}^{\text {geo }}$ and R-charges that were obtained from geometric c-extremization (see section 6.3.3.1). We have taken triples $a, b, c$ with $-150<a<0, b<150$ and satisfying the conditions 6.123. This resulted in 48289 possible triples to analyze. In order to compare the two central charges we have to continue the domain of the result on the c-extremization side to allow for negative values of $a$. Furthermore, to compute $c_{R}^{\text {geo }}$ and the R-charges on the c-extremization side one needs the values of $m_{1}, n_{2}$ and $n_{3}$. These can be calculated from the solution via the fluxes (6.94)- 6.97) and the relations 6.45, 6.52, i.e.

$$
m_{1}=\frac{1}{N} M_{\xi_{+}}, \quad n_{2}=-\frac{1}{N}\left(M_{\xi_{-}}+a k M_{\eta_{-}}-a l M_{\xi_{+}}\right), \quad n_{3}=-\frac{1}{N}\left(b M_{\eta_{-}}+c M_{\xi_{+}}\right) .
$$

The factor $L^{4} /\left(g_{s} \ell_{s}^{4}\right)$ appearing in the fluxes is expressed in terms of $N$ via 6.91. We found matching central charges and R-charges for all triples analyzed. For

\footnotetext{
${ }^{15}$ We have excluded values where $c=d$ since this is in fact the $Y^{p, q}$ limit.
} 
purposes of illustration explicit values for $m_{1}, n_{2}, n_{3}, c_{\text {sugra }}=c_{R}^{\text {geo }}$ and the R-charges for 3 triples with $a<0$ can be found in table 6.2 .

When $a>0$ one can still solve the regularity conditions $\sqrt{6.120}$ for $u$ and $v$. However, one does not find a solution in the domain 6.119. Even though the solution is not regular one can proceed and compute its central charges and Rcharges. These values do still match with the field theory side, however it turns out that always either the central charge or one of the R-charges is negative. To illustrate this, we have also presented three triples with positive $a$ in table 6.2 It is an opportune moment to remind the reader that the necessity of negative $a$ can also be shown at the field theory side, namely the cases considered in section 6.2 .2 did not simultaneously have positive central charge and positive R-charges for $a>0$ for any choice of twist parameters.

We thus only obtain physically meaningful results when $a$ is negative. Note that in the field theory this analytic continuation is ill-defined if one considers the reduction from four dimensions since $a$ is related to the multiplicity of a particular field. Ignoring this subtlety we did find that the expressions for the central charge and R-charges computed at the field theory side formally agree with the expressions in this section. We emphasize that the supergravity solutions for negative $a$ constructed here have no pathologies and are singularity free, globally well-defined solutions. They are not regular for $a>0$ however. This result seems to suggest that one cannot construct a flow solution from $\mathrm{AdS}_{5}$ to $\mathrm{AdS}_{3} \times \mathrm{T}^{2}$ in a consistent truncation of $L^{a, b, c}$ and it would be interesting to understand why this is the case. Moreover, identifying the field theory dual to these solutions remains an open

\begin{tabular}{ccccccccc}
\hline$(a, b, c)$ & $m_{1}$ & $n_{2}$ & $n_{3}$ & $c_{R}$ & $R_{\xi_{-}}$ & $R_{\xi_{+}}$ & $R_{\eta_{-}}$ & $R_{\eta_{+}}$ \\
\hline$(-8,116,97)$ & -0.49 & 798.36 & 211.30 & -335.60 & 1.31 & 0.33 & 0.16 & 0.20 \\
$(-27,91,62)$ & -4.42 & 2681.15 & 411.61 & -196.26 & 1.13 & 0.84 & 0.01 & 0.02 \\
$(-53,139,79)$ & -3.36 & -7722.81 & 460.05 & -270.34 & 1.16 & 0.80 & 0.02 & 0.03 \\
$(7,133,111)$ & 0.26 & 55.57 & 185.60 & -387.21 & 1.32 & -0.26 & 0.49 & 0.44 \\
$(25,139,108)$ & 0.70 & -315.87 & 193.15 & -246.98 & 1.25 & -0.81 & 0.85 & 0.71 \\
$(50,122,119)$ & 12.04 & -2824.87 & 167.15 & 53355.54 & -2.37 & -14.06 & 13.69 & 4.75 \\
\hline
\end{tabular}

Table 6.2: Values of $m_{1}, n_{2}, n_{3}$ (divided by $C$ ), $c_{R}^{\text {geo }}=c_{\text {sugra }}\left(\right.$ divided by $C N^{2}$ ) and the R-charges (divided by $N$ ) for certain triples $a, b$ and $c$. The triples with negative $a$ correspond to globally well-defined solutions; note that $C<0$ and therefore the central charges are positive. The triples with positive $a$ always have at least one negative R-charge. 
problem. A similar discrepancy has previously been observed for direct products $Y_{7}=\mathrm{T}^{2} \times Y^{p, q} 16 L^{a, b, c}$ and for a specific fibration of $Y^{p, q} \hookrightarrow Y_{7} \rightarrow \mathrm{S}^{2} 132,204,205$. The $Y^{p, q}$ field theory is characterized by integers $p>q>0$ whilst the solutions are characterized by integers $q>p>0$

\subsubsection{Sphere fibrations}

Let us now turn our attention to the solutions consisting of a fibration over a two-sphere. The analysis in this case and in the higher genus Riemann surfaces, that we tackle in the subsequent section, are even more cumbersome than the analysis presented in the previous section for the torus. The torus case was easier because we could find a coordinate transformation for the auxiliary roots which got rid of a square root in the physical roots. It also made the parameter $C$ drop out of the physical roots and the regularity conditions. For the $\kappa= \pm 1$ cases we have not found such a coordinate transformation and (therefore) also not found a solution that expresses $\xi_{*}$ and $\eta_{*}$ as function of $a, b, c$ and $C$. Nevertheless we can still make progress and identify ranges for the parameters which give global solutions. We shall supplement the ranges of the parameters with some explicit numerical computations which show that the results obtained here agree with the results obtained from the field theory and geometric dual of c-extremization. Again, this matching is up to the domains of the integers $a, b, c$ characterizing the gravity solution and the field theory. The analysis is largely analogous to the previous case and we will thus be brief in places. In particular the matching of the dictionary in equation 6.124 is the same.

For the fibrations over a two-sphere it is possible to find solutions for both $D= \pm 1$. It is also possible to rewrite the regularity conditions such that the integers $a$ and $c$ are expressed in terms of the auxiliary roots and $C$ as in 6.120 . However, these expressions are exceedingly tedious and we therefore refrain from presenting them in this chapter. Despite this it can be algebraically proven that the integers $a, b, c$ and $d$ are necessarily of the form 6.123 in order to obtain globally regular solutions. This is the same as in the torus case studied above and the same discussion appearing there is equally applicable here.

We now focus on the case that $D=1$ to provide a few more details. First we make the coordinate transformations

$$
\eta_{*}=-\frac{C(2+v)}{4}, \quad \xi_{*}=-C\left(\frac{2}{u}+1\right) .
$$

\footnotetext{
$\overline{{ }^{16} Y^{p, q}}$ are again, in abuse of notation, the analogues of the Sasaki-Einstein manifolds $Y^{p, q}$. ${ }^{17} \mathrm{We}$ also found this for the more general $Y^{p, q}$ fibrations we studied in an appendix of 4 .
} 
We now use the equations 6.113 to express the physical roots and parameters $A$ and $B$ as

$$
\begin{aligned}
& \eta_{ \pm}= \frac{2(2-C) v-C v^{2} \pm 2 \sqrt{4 v^{2}+C^{2}(v+2)^{2}(v-1)-C\left(v^{3}+6 v^{2}+4 v-8\right)}}{(v-2)^{2}} \\
& \xi_{ \pm}= \frac{1}{8}\left(4 u+u^{2}-C\left(8+6 u+u^{2}\right)\right. \\
&\left.\quad \quad \pm \sqrt{u\left[(C(2+u)-u)\left(C\left(16+10 u+u^{2}\right)-(4+u)^{2}\right)\right]}\right), \\
& A=\frac{(u+2)^{2}(u-C(u+2))}{4 u}, \\
& B=\frac{(v+2)^{2}(C(v+2)-4)}{4(v-2)^{2}} .
\end{aligned}
$$

One of the domains, where the solution is globally regular, is given by

$$
0<v<1, \quad 0<u<v, \quad \frac{v^{2}}{v^{2}+v-2}<C<\frac{u(u(v-2)+4 v)}{u^{2}(v-2)-(v-2)^{2}+u(6 v-4)} .
$$

We have solved the regularity conditions numerically using this domain for a large number of triples $a, b, c$ satisfying 6.123. For these triples we also compared the results for the central charges and R-charges obtained from the solutions to the field theory and geometric dual results, as we did for the torus case. We have presented the results for three triples in table 6.3. For these triples we fixed $v=0.5$ and solved the regularity conditions for $u$ and $C$ within the domain (6.127).

\begin{tabular}{ccccccccc}
\hline$(a, b, c)$ & $m_{1}$ & $n_{2}$ & $n_{3}$ & $c_{R}$ & $R_{\xi_{-}}$ & $R_{\xi_{+}}$ & $R_{\eta_{-}}$ & $R_{\eta_{+}}$ \\
\hline$(-19,29,8)$ & -2.38 & 177.63 & -24.21 & 4.49 & 1.30 & 0.68 & 0.01 & 0.01 \\
$(-8,26,15)$ & -0.71 & 40.41 & -17.38 & 9.09 & 1.52 & 0.38 & 0.05 & 0.06 \\
$(-1,25,16)$ & -0.07 & 8.64 & -12.82 & 14.88 & 1.60 & 0.05 & 0.18 & 0.18 \\
\hline
\end{tabular}

Table 6.3: Values of $m_{1}, n_{2}, n_{3}, c_{R}^{\text {geo }}=c_{\text {sugra }}$ (divided by $N^{2}$ ), and the R-charges (divided by $N$ ) for certain triples $a, b, c$ and $\kappa=1$. Note that the central charges and R-charges are all positive.

\subsubsection{Higher genus Riemann surface fibrations}

In this final section we will study the regularity of solutions for which the genus is $g>1$. This case is distinct from the two previous cases in that it is possible to 
find globally regular solutions for which the integers $a, b, c$ and $d$ are all positive as one would expect from the naive comparison with the $L^{a, b, c}$ Sasaki-Einstein solutions. Additionally, we find that there are also regular solutions for integers satisfying $a<0<d \leq c \leq b$. We shall therefore split this section in two parts to give examples of both cases. We furthermore find that there are no global solutions for $D=-1$; global solutions only exist for $D=1$.

Positive $\boldsymbol{a}$ solutions. We first make the change of parameters

$$
\eta_{*}=-C v, \quad \xi_{*}=-C u .
$$

The physical roots and parameters $A$ and $B$ in terms of these variables are

$$
\begin{aligned}
\eta_{ \pm} & =\frac{1+(C-2) v-2 C v^{2} \pm \sqrt{2 C v\left(2 v^{2}-1\right)+(2 v-1)^{2}+C^{2} v^{2}(4 v-3)}}{2(v-1)^{2}}, \\
\xi_{ \pm} & =\frac{1+(C-2) u-2 C u^{2} \pm \sqrt{2 C u\left(2 u^{2}-1\right)+(2 u-1)^{2}+C^{2} u^{2}(4 u-3)}}{2(u-1)^{2}}, \\
A & =-\frac{u^{2}(C u+1)}{(u-1)^{2}}, \\
B & =\frac{v^{2}(C v+1)}{(v-1)^{2}} .
\end{aligned}
$$

One of the domains where the solutions can be globally regular is given by

$$
\begin{gathered}
\frac{2 v}{2 v-1}<u<\frac{4 v-3}{4 v-4}, \quad \frac{9}{8}<v<\frac{1}{4}(1+\sqrt{13}), \\
\frac{2 u v-u-v}{u^{2}+u v-2 u^{2} v+v^{2}-2 u v^{2}+u^{2} v^{2}}<C<\frac{-1+4 v-4 v^{2}}{4 v^{2}-3 v} .
\end{gathered}
$$

For this domain the integers $a, b, c, d$ corresponding to global regular solutions are also all positive and satisfy the field theoretic conditions (6.1), i.e.

$$
0<a \leq d \leq c \leq b, \quad \operatorname{gcd}(a, c)=\operatorname{gcd}(a, d)=\operatorname{gcd}(b, c)=\operatorname{gcd}(b, d)=1 .
$$

We can again compare the solutions to the field theory by numerically solving the regularity conditions and computing the central charge and R-charges. We have found matching values for all cases we checked. This is strong evidence for new dualities where the gravity side is given by the solutions we found and the field theories are twisted compactifications of the $4 \mathrm{~d} L^{a, b, c}$ quiver gauge theories. We have again given a few examples for explicit triples in table 6.4. Here we have fixed $v=1.13$ and solved the regularity conditions for $u$ and $C$ taking values according to 6.130 . 


\begin{tabular}{ccccccccc}
\hline$(a, b, c)$ & $m_{1}$ & $n_{2}$ & $n_{3}$ & $c_{R}$ & $R_{\xi_{-}}$ & $R_{\xi_{+}}$ & $R_{\eta_{-}}$ & $R_{\eta_{+}}$ \\
\hline$(1,19,11)$ & 0.03 & -6.14 & -15.77 & 53.48 & 0.88 & 0.29 & 0.40 & 0.42 \\
$(8,26,23)$ & 0.13 & 61.06 & -22.14 & 87.13 & 0.86 & 0.67 & 0.18 & 0.29 \\
$(14,22,19)$ & 0.17 & -91.44 & -20.50 & 90.53 & 0.82 & 0.75 & 0.20 & 0.22 \\
\hline
\end{tabular}

Table 6.4: Values of $m_{1}, n_{2}$ and $n_{3}$ (divided by $g-1$ ), $c_{R}^{\text {geo }}=c_{\text {sugra }}$ (divided by $(g-1) N^{2}$ ) and the R-charges (divided by $N$ ) for certain triples $a, b, c$ with positive $a$ and $\kappa=-1$. Observe that the central charges and R-charges are all positive.

Negative $\boldsymbol{a}$ solutions. To find globally regular solutions with $a<0$ one can make the parameter redefinitions

$$
\eta_{*}=-\frac{v}{C^{\prime}}, \quad \xi_{*}=-\frac{1}{C^{\prime} u}, \quad C=\frac{1}{C^{\prime}}
$$

Using the conditions 6.113 we express the physical roots and $A, B$ as

$$
\begin{aligned}
\eta_{ \pm} & =\frac{C^{\prime}+v-2 C v-2 v^{2} \mp \sqrt{C^{\prime 2}(2 v-1)^{2}+v^{2}(4 v-3)+2 C^{\prime} v\left(2 v^{2}-1\right)}}{2 C^{\prime}(v-1)^{2}} \\
\xi_{ \pm} & =\frac{-2+u-2 C u+C u^{2} \mp \sqrt{u\left(1+C^{\prime} u\right)\left(4-3 u+C^{\prime}(u-2)^{2}\right)}}{2 C^{\prime}(u-1)^{2}} \\
A & =-\frac{1+C^{\prime} u}{C^{\prime} u(u-1)^{2}}, \\
B & =\frac{v^{2}\left(C^{\prime}+v\right)}{C^{\prime}(v-1)^{2}} .
\end{aligned}
$$

A range of parameters for which the solutions can be globally regular is given by

$$
\frac{3}{4}<v<1, \quad 0<u<\frac{2(v-1)^{2}}{v(2 v-1+\sqrt{4 v-3})}, \quad-\frac{v(4 v-3)}{(2 v-1)^{2}}<C^{\prime}<0 .
$$

For this domain we can show that the solutions of the regularity conditions necessarily have $a<0$ with the remaining integers all positive satisfying 6.123). We can indeed numerically solve the regularity conditions for explicit values of $a, b$ and $c$ using these domains. The resulting solutions can again be compared to the field theory by computing their central charge and R-charges. Three examples can be found in table 6.5. For these triples we took $v=0.8$ and solved the regularity conditions for $u$ and $C^{\prime}$ obeying the bounds (6.134). 
6 Twisted $\mathcal{N}=1$ SCFTs and their $A d S_{3}$ duals

\begin{tabular}{ccccccccc}
\hline$(a, b, c)$ & $m_{1}$ & $n_{2}$ & $n_{3}$ & $c_{R}$ & $R_{\xi_{-}}$ & $R_{\xi_{+}}$ & $R_{\eta_{-}}$ & $R_{\eta_{+}}$ \\
\hline$(-19,29,8)$ & 14.38 & 1685.99 & -232.51 & 103.86 & 1.08 & 0.90 & 0.01 & 0.01 \\
$(-8,26,15)$ & 3.93 & 355.01 & -161.14 & 175.14 & 1.18 & 0.71 & 0.04 & 0.07 \\
$(-1,25,16)$ & 0.21 & 37.82 & -76.81 & 167.57 & 1.25 & 0.15 & 0.29 & 0.31 \\
\hline
\end{tabular}

Table 6.5: Values of $m_{1}, n_{2}$ and $n_{3}$ (divided by $g-1$ ), $c_{R}^{\text {geo }}=c_{\text {sugra }}$ (divided by $(g-1) N^{2}$ ) and the R-charges (divided by $N$ ) for certain triples $a, b, c$ with negative $a$ and $\kappa=-1$. Observe that the central charges and R-charges are all positive.

\subsection{Discussion}

The solutions we have constructed in this chapter are of the form $\operatorname{AdS}_{3} \times Y_{7}$ where $L^{a, b, c} \hookrightarrow Y_{7} \rightarrow \Sigma_{g}$. We have not found the most general solution of this form since it is lacking two parameters in comparison to the field theory. The ansatz we have used depends on four unknown functions $\mathcal{F}, \mathcal{G}, \mathcal{H}$ and $\mathcal{L}$ and by requiring the recovery of the universal twist solution the form of $\mathcal{H}$ and $\mathcal{L}$ is fixed. It is therefore expected, that a suitable generalization of the ansätze for the functions $\mathcal{F}$ and $\mathcal{G}$ accounts for the missing parameters.

We have obtained generic expressions for the fluxes, central charge and R-charges without requiring a solution of the master equation. Recall that the central charge agrees off-shell for c-extremization and its geometric dual [205,212]. It would be interesting to see if one can also obtain a matching to the field theory with our off-shell expressions for the charges.

Let us stress again that the matching of central charges and R-charges between field theory and the solutions is only a formal matching for the $\mathrm{S}^{2}, \mathrm{~T}^{2}$ and $a<0$ case of $\Sigma_{g>1}$. While the field theory results are valid for $a>0$, globally regular solutions necessarily have $a<0$ in the $\mathrm{S}^{2}$ and $\mathrm{T}^{2}$ cases, whilst they can have both $a>0$ and $a<0$ for $g>1$ compactifications. For the field theories compactified on $\mathrm{S}^{2}$ and $\mathrm{T}^{2}$ with $a>0$ we find at least one negative R-charge, which is inconsistent with representation theory of the superconformal algebra. This is evidence that the compactified $4 \mathrm{~d} \mathcal{N}=1$ theories on these spaces do not flow to an IR fixed point. It would be of great interest to study the RG flow trajectories of the compactified $4 \mathrm{~d}$ theories from first principles, e.g with field theoretical methods or holographic RG flow across dimensions as was done in 238.

Nevertheless, even for the cases with $a<0$, we found well-defined supersymmetric $\mathrm{AdS}_{3}$ solutions of type IIB supergravity, which according to the AdS/CFT correspondence should have $2 \mathrm{~d}$ SCFT duals. The formulation of these SCFTs and 
if they admit an interpretation as descending via compactification from higher dimensional SCFTs remains an interesting puzzle.

\section{Appendices}

\section{A Ansatz analysis details}

As explained in the main text, the metric ansatz always admits a closed nondegenerate two-form, $J$, which may be viewed as the almost complex structure of the metric. Despite this, the metric is not a complex manifold for arbitrary $\mathcal{H}$ and $\mathcal{L}$. The vanishing of the Nijenhuis tensor, and thus the integrability of the almost complex structure still needs to be imposed. This is most easily enforced by requiring that the exterior derivative of the holomorphic $(3,0)$ form $\Omega$, compatible with the chosen almost complex structure, satisfies

$$
\mathrm{d} \Omega=\mathrm{i} P \wedge \Omega
$$

for some one-form $P$. The one-form $P$ is the canonical Ricci-form potential defined by the Kähler metric satisfying $\mathrm{d} P=\rho$, with $\rho=\frac{1}{2} R_{i j k l} J^{k l} \mathrm{~d} x^{i} \wedge \mathrm{d} x^{j}$ the Ricci-form of the Kähler metric. After a few pages of computation one finds that the necessary and sufficient conditions for the integrability of the complex structure are the following five non-trivially coupled differential equations

$$
\begin{aligned}
\mathcal{F}(\xi)^{2} \partial_{\xi}^{2} \mathcal{H}(\eta, \xi) & =\mathcal{G}(\eta)^{2} \partial_{\eta}^{2} \mathcal{H}(\eta, \xi), \\
\mathcal{F}(\xi)^{2} \partial_{\xi}^{2} \mathcal{L}(\eta, \xi) & =\mathcal{G}(\eta)^{2} \partial_{\eta}^{2} \mathcal{L}(\eta, \xi), \\
(\eta-\xi) \mathcal{G}(\eta) \partial_{\eta}^{2} \mathcal{H}(\eta, \xi) & \left.=-\mathcal{F}(\xi)\left[\left(\partial_{\eta}-\partial_{\xi}\right) \mathcal{H}(\eta, \xi)+(\eta-\xi) \partial_{\eta} \partial_{\xi} \mathcal{H}(\eta, \xi)\right)\right] \\
(\eta-\xi) \mathcal{G}(\eta) \partial_{\eta}^{2} \mathcal{L}(\eta, \xi) & \left.=-\mathcal{F}(\xi)\left[\left(\partial_{\eta}-\partial_{\xi}\right) \mathcal{L}(\eta, \xi)+(\eta-\xi) \partial_{\eta} \partial_{\xi} \mathcal{L}(\eta, \xi)\right)\right] \\
\partial_{\eta} \partial_{\xi} \mathcal{H}(\eta, \xi)\left(\partial_{\eta}-\partial_{\xi}\right) \mathcal{L}(\eta, \xi) & =\partial_{\eta} \partial_{\xi} \mathcal{L}(\eta, \xi)\left(\partial_{\eta}-\partial_{\xi}\right) \mathcal{H}(\eta, \xi)
\end{aligned}
$$

As a by-product of this analysis the Ricci-form potential is found to be

$$
\begin{aligned}
P= & P_{g}-\frac{\partial_{\xi}(\mathcal{F}(\xi) \mathcal{H}(\eta, \xi))}{2(\eta-\xi) \mathcal{H}(\eta, \xi)}\left(\mathrm{d} \phi+\partial_{\xi} \mathcal{L}(\eta, \xi) \mathrm{d} \psi+\partial_{\xi} \mathcal{H}(\eta, \xi) \mathcal{A}_{g}\right) \\
& -\frac{\partial_{\eta}(\mathcal{G}(\eta) \mathcal{H}(\eta, \xi))}{2(\eta-\xi) \mathcal{H}(\eta, \xi)}\left(\mathrm{d} \phi+\partial_{\eta} \mathcal{L}(\eta, \xi) \mathrm{d} \psi+\partial_{\eta} \mathcal{H}(\eta, \xi) \mathcal{A}_{g}\right) \\
& -\frac{\mathcal{F}(\xi) \partial_{\xi}^{2} \mathcal{L}(\eta, \xi)+\mathcal{G}(\eta) \partial_{\eta}^{2} \mathcal{L}(\eta, \xi)}{\eta-\xi} \mathrm{d} \psi-\frac{\mathcal{F}(\xi) \partial_{\xi}^{2} \mathcal{H}(\eta, \xi)+\mathcal{G}(\eta) \partial_{\eta}^{2} \mathcal{H}(\eta, \xi)}{\eta-\xi} \mathcal{A}_{g},
\end{aligned}
$$


where $P_{g}$ and $\mathcal{A}_{g}$ are the Ricci-form potential and Kähler form potential on the Riemann surface respectively. We are unable to solve 6.136 in full generality, but it is not too difficult to see that a family of solutions is given by

$$
\mathcal{H}(\eta, \xi)=\alpha_{0}+\alpha_{1}(\eta+\xi)+\alpha_{2} \eta \xi, \quad \mathcal{L}(\eta, \xi)=\beta_{0}+\beta_{1}(\eta+\xi)+\beta_{2} \eta \xi .
$$

The three constants in $\mathcal{L}$ are trivial and can be removed by coordinate transformations of the angular coordinates and in this manner one can set $\beta_{0}=\beta_{1}=0$ and $\beta_{2}=1$. The universal twist solution is obtained by taking $\mathcal{L}=\eta \xi$ and therefore it seems reasonable that this choice of solution is the correct one for our purposes. Once we have fixed this, $\mathcal{H}$ is fixed to take the form in 6.138. Namely, substituting $\mathcal{L}=\eta \xi$ into the last equation in 6.136 implies $\mathcal{H}$ takes the form presented in 6.138 .

\section{B Effective coordinate systems}

In this appendix we state the effective coordinate systems which are needed to perform the integrations over the angular parts. For an explanation of how to construct the transformation 6.83 see e.g. 217. From this relation it is easy to derive the corresponding coordinate transformation 6.88 which was needed for the computation of the flux $N$ and for integrations over the degeneration surface corresponding to $k_{\eta_{+}}$. We now state the transformations needed for the integrations over the other three degeneration surfaces.

$\boldsymbol{\eta}=\boldsymbol{\eta}_{-}$. The vectors generating the effective torus action are now related to the Killing vectors via

$$
\left(\begin{array}{c}
k_{\eta_{-}} \\
k_{\eta_{+}} \\
l_{\xi_{-}} \\
l_{\xi_{+}}
\end{array}\right)=\left(\begin{array}{ccc}
1 & 0 & 0 \\
1 & b k & a \\
1 & b l & d \\
1 & 1 & 0
\end{array}\right)\left(\begin{array}{c}
\partial_{\psi_{1}} \\
\partial_{\psi_{2}} \\
\partial_{\psi_{3}}
\end{array}\right),
$$

where $k, l$ are solutions of $d k-a l=1$. The corresponding coordinate transformation is then given by

$$
\begin{aligned}
& z=\psi_{1} \\
& \psi=-\frac{2}{\mathcal{G}_{-}^{\prime}} \psi_{1}+2\left(\frac{1}{\mathcal{F}_{+}^{\prime}}+\frac{1}{\mathcal{G}_{-}^{\prime}}\right) \psi_{2}+2\left(\frac{k}{\mathcal{F}_{-}^{\prime}}+\frac{l}{\mathcal{G}_{+}^{\prime}}+\frac{k-l}{\mathcal{G}_{-}^{\prime}}\right) \psi_{3}, \\
& \phi=\frac{2 \eta_{-}}{\mathcal{G}_{-}^{\prime}} \psi_{1}-2\left(\frac{\xi_{+}}{\mathcal{F}_{+}^{\prime}}+\frac{\eta_{-}}{\mathcal{G}_{-}^{\prime}}\right) \psi_{2}-2\left(\frac{k \xi_{-}}{\mathcal{F}_{-}^{\prime}}+\frac{l \eta_{+}}{\mathcal{G}_{+}^{\prime}}+\frac{(k-l) \eta_{-}}{\mathcal{G}_{-}^{\prime}}\right) \psi_{3} .
\end{aligned}
$$


Since $k_{\eta_{-}}=\partial_{\psi_{1}}$, at the $\eta_{-}$degeneration surface the $\mathrm{T}^{3}$ will degenerate to a $\mathrm{T}^{2}$ which has as basis of the effective action the coordinates $\psi_{2}$ and $\psi_{3}$.

$\boldsymbol{\xi}=\boldsymbol{\xi}_{+} \cdot$ We now have the relation

$$
\left(\begin{array}{c}
l_{\xi_{+}} \\
l_{\xi_{-}} \\
k_{\eta_{-}} \\
k_{\eta_{+}}
\end{array}\right)=\left(\begin{array}{ccc}
1 & 0 & 0 \\
1 & -d k & -c \\
1 & -d l & -a \\
1 & 1 & 0
\end{array}\right)\left(\begin{array}{l}
\partial_{\psi_{1}} \\
\partial_{\psi_{2}} \\
\partial_{\psi_{3}}
\end{array}\right),
$$

where $k, l$ solve the relation $c l-a k=1$. The coordinate transformation that follows from this relation is

$$
\begin{aligned}
& z=\psi_{1} \\
& \psi=\frac{2}{\mathcal{F}_{+}^{\prime}} \psi_{1}-2\left(\frac{1}{\mathcal{F}_{+}^{\prime}}+\frac{1}{\mathcal{G}_{+}^{\prime}}\right) \psi_{2}-2\left(\frac{k}{\mathcal{G}_{-}^{\prime}}+\frac{l}{\mathcal{F}_{-}^{\prime}}+\frac{k-l}{\mathcal{F}_{+}^{\prime}}\right) \psi_{3}, \\
& \phi=-\frac{2 \xi_{+}}{\mathcal{F}_{+}^{\prime}} \psi_{1}+2\left(\frac{\xi_{+}}{\mathcal{F}_{+}^{\prime}}+\frac{\eta_{+}}{\mathcal{G}_{+}^{\prime}}\right) \psi_{2}+2\left(\frac{k \eta_{-}}{\mathcal{G}_{-}^{\prime}}+\frac{l \xi_{-}}{\mathcal{F}_{-}^{\prime}}+\frac{(k-l) \xi_{+}}{\mathcal{F}_{+}^{\prime}}\right) \psi_{3},
\end{aligned}
$$

where again $\psi_{2}$ and $\psi_{3}$ are the coordinates needed for integration over the surface corresponding to $l_{\xi_{+}}$.

$\boldsymbol{\xi}=\boldsymbol{\xi}_{-}$. This time the relation between the Killing vectors and the vectors generating an effective torus action is given by

$$
\left(\begin{array}{c}
l_{\xi_{-}} \\
l_{\xi_{+}} \\
k_{\eta_{-}} \\
k_{\eta_{+}}
\end{array}\right)=\left(\begin{array}{ccc}
1 & 0 & 0 \\
1 & d k & c \\
1 & -d l & b \\
1 & 1 & 0
\end{array}\right)\left(\begin{array}{c}
\partial_{\psi_{1}} \\
\partial_{\psi_{2}} \\
\partial_{\psi_{3}}
\end{array}\right),
$$

in which $k, l$ solve $c l+b k=1$. The coordinate transformation is then equal to

$$
\begin{aligned}
& z=\psi_{1} \\
& \psi=\frac{2}{\mathcal{F}_{-}^{\prime}} \psi_{1}-2\left(\frac{1}{\mathcal{F}_{-}^{\prime}}+\frac{1}{\mathcal{G}_{+}^{\prime}}\right) \psi_{2}-2\left(\frac{k}{\mathcal{G}_{-}^{\prime}}-\frac{l}{\mathcal{F}_{+}^{\prime}}+\frac{k+l}{\mathcal{F}_{-}^{\prime}}\right) \psi_{3}, \\
& \phi=-\frac{2 \xi-}{\mathcal{F}_{-}^{\prime}} \psi_{1}+2\left(\frac{\xi_{-}}{\mathcal{F}_{-}^{\prime}}+\frac{\eta_{+}}{\mathcal{G}_{+}^{\prime}}\right) \psi_{2}+2\left(\frac{k \eta_{-}}{\mathcal{G}_{-}^{\prime}}-\frac{l \xi_{+}}{\mathcal{F}_{+}^{\prime}}+\frac{(k+l) \eta_{-}}{\mathcal{F}_{-}^{\prime}}\right) \psi_{3} .
\end{aligned}
$$

As in the previous cases $\psi_{2}$ and $\psi_{3}$ are the relevant coordinates for the corresponding degenerate surface. 



\section{Summary}

In this thesis we have examined the entropy of certain classes of black holes in string theory. As shown by Bekenstein and Hawking in the 1970s, black holes have entropy which scales with the area of their event horizon. For thermodynamic systems entropy corresponds to the number of microstates corresponding to a certain macrostate. For a long time it was not clear what these microstates could be for black holes. To understand them one needs a theory of quantum gravity. One candidate for this is string theory which uses strings instead of point particles to describe the fundamental degrees of freedom. Spacetime in string theory is ten-dimensional of which six dimensions should be small in order to make our world seem four-dimensional. Being able to account for the microstates of black holes serves as a test of string theory. In the 1990s it was shown that this is indeed possible. Black holes arise as higher dimensional objects, branes, wrapped in the compact dimensions. These branes can be described in two different ways. The first one is by a solution of a supergravity theory which is a supersymmetric extension of general relativity. The second one is by the worldvolume theory of the brane which corresponds to excitations of open strings connected to the brane. It is this worldvolume theory we can use to count microstates. The black holes we consider have an uplift to a black string. The worldvolume theory can then be compactified to the degrees of freedom of this string. This results in a $2 \mathrm{~d}$ theory which flows to a $2 \mathrm{~d}$ superconformal field theory (SCFT) in the IR. The entropy is then via the Cardy formula expressed in characteristic data of this SCFT, namely in its central charges and levels. The Cardy formula also captures corrections to the leading area law. To reproduce this entropy from the macroscopic supergravity solution description, we use that the near-horizon geometry of a black string is given by a geometry with an $\mathrm{AdS}_{3}$ factor. The AdS/CFT correspondence tells us that the central charges and levels correspond to the coefficients of certain Chern-Simons terms in the $3 \mathrm{~d}$ action. We can thus determine the central charges and levels by reducing the relevant supergravity action in the near-horizon geometry of the black string to an effective $3 \mathrm{~d}$ action and reading off the coefficients of the relevant Chern-Simons terms. It turns out that to also capture degrees of freedom living outside of the horizon one actually has to perform this reduction at asymptotic infinity. 
In this thesis we have applied the techniques outlined above to black string solutions in compactifications of F-theory on an elliptically fibered Calabi-Yau threefold or of type IIB string theory on K3. The black string arises as a D3-brane wrapped on a curve in either the base of the Calabi-Yau threefold or in K3. The Ftheory compactifications result in effective six-dimensional $\mathcal{N}=(1,0)$ supergravity theories of which the details are determined by topological data of the threefold These theories have a gravity multiplet, tensor multiplets, hypermultiplets and vector multiplets, but for the black string solutions of interest we always take the hyperscalars constant and the vectors vanishing. Therefore, the relevant solutions solve $6 \mathrm{~d} \mathcal{N}=(1,0)$ supergravity coupled to tensor multiplets. The latter theory is also realized as a truncation of the $6 \mathrm{~d} \mathcal{N}=(2,0)$ supergravity theory which one obtains when compactifying type IIB supergravity on K3. In chapter 2 we examined supersymmetric solutions of $6 \mathrm{~d} \mathcal{N}=(1,0)$ supergravity coupled to tensor multiplets. We first derived a general local form for supersymmetric solutions after which we considered several special cases in which the resulting equations could be solved explicitly. This way we in particular derived expressions for the black string solutions we examined in chapters 35 .

In chapter 3 we considered black string solutions in F-theory that have a TaubNut space transversely. This transverse space is a circle fibration over $\mathbb{R}^{3}$ which is why this setting corresponds to four-dimensional black holes. We furthermore only considered compactifications of F-theory on elliptically fibered Calabi-Yau threefolds in which the degenerations of the fibers do not lead to singularities of the whole fibration. This corresponds to $6 \mathrm{~d}$ supergravity theories without vector multiplets. From the six-dimensional supergravity perspective we reproduced the central charges and levels of the SCFT, which were calculated from a microscopic viewpoint via $\mathrm{M}$-theory in 75 . We identified and included a shift in the identification of the macroscopic with the microscopic charges caused by the Green-Schwarz term in the pseudo-action. Performing the reduction at asymptotic infinity and the inclusion of one-loop Chern-Simons terms arising from integrating out massive Kaluza-Klein modes are the crucial ingredients to obtain the non-trivial matching.

In chapter 4 we generalized the macroscopic computations of chapter 3 in two ways. Firstly, we replaced the transverse Taub-NUT space by an arbitrary asymptotically locally Euclidean (ALE) or asymptotically locally flat (ALF) space. We have computed the corresponding central charges and levels using the techniques outlined above. Secondly, we considered Calabi-Yau threefold fibrations in which the degenerations do lead to singularities of the whole fibration. This results in vector multiplets and charged hypermultiplets in the $6 \mathrm{~d} \mathcal{N}=(1,0)$ supergravity theory. 
The vector multiplets furnish a gauge symmetry of the $6 \mathrm{~d}$ bulk theory which from the point of view of the string acts as a flavour symmetry of the worldvolume theory living on the degrees of freedom of the string. These non-abelian symmetries have associated levels which we determined using the $6 \mathrm{~d}$ supergravity theory. As before they correspond to coefficients of Chern-Simons terms in the effective $3 \mathrm{~d}$ action. We furthermore showed that the vector multiplets and charged hypermultiplets do not change the expressions of the earlier computed central charges and levels in terms of the microscopic charges and geometric data.

For the settings of chapter 4 the macroscopic expressions for the central charges and levels can only be matched with a microscopic computation when the transverse space is either equal to Taub-NUT or to $\mathbb{R}^{4}$. This is why in chapter 5 we studied black strings with ALE or ALF spaces transversely arising in compactifications of type IIB string theory on K3. We again computed the central charges and levels using the $6 \mathrm{~d}$ supergravity theory. When the space transverse to the string is either ALE or Taub-NUT there is a complementary microscopic computation. When the transverse space is given by Taub-NUT the microscopic computation can be done via the dual M-theory picture, just as in the F-theory setting. When the transverse space is ALE we took the worldvolume theory of a D3-brane probing this ALE space, which is a $4 \mathrm{~d} \mathcal{N}=2$ quiver gauge theory, and compactified it on the curve the brane is wrapped upon. In order to preserve $\mathcal{N}=(0,4)$ supersymmetry in the $2 \mathrm{~d}$ theory this requires a particular topological twist to be performed. The central charges and levels of the SCFT correspond to certain anomaly coefficients and can by 't Hooft anomaly matching be computed in the UV-theory. We found a perfect matching to the macroscopic computations.

In chapter 6 we changed gears a little bit. We have studied the IR-limits of compactifications of the $4 \mathrm{~d} L^{a, b, c} \mathcal{N}=1$ quiver gauge theories on a Riemann surface with fluxes of global symmetries switched on. Assuming they flow to an $\mathcal{N}=(0,2)$ SCFT we have computed the central charges and R-charges of this IR fixed point in three different ways. Field theoretically we computed the latter by utilizing the technique of c-extremization. With knowledge of UV data it is then possible to calculate the central charge and R-charges by virtue of 't Hooft anomaly matching. The second way is the recently found geometric dual formulation of c-extremization. This technique only depends on topological data of the solution and assumes the existence of a supergravity solution. Lastly, we have constructed explicit type IIB supergravity solutions dual to $2 \mathrm{~d} \mathcal{N}=(0,2)$ SCFTs. These solutions contain an $\mathrm{AdS}_{3}$ factor and can be thought of as the near-horizon geometry of black strings that are asymptotically $\mathrm{AdS}_{5} \times L^{a, b, c}$. We used the solutions to compute the 
corresponding central charges and R-charges directly. We found an exact matching between the field theoretic and geometric values. Since the central charge is related to the entropy density of the strings, the matching between the supergravity and field theory sides can be viewed as a microscopic counting of the degrees of freedom of the string. 


\section{Samenvatting}

In dit proefschrift hebben we de entropie van bepaalde klassen van zwarte gaten in de snaartheorie onderzocht. Zoals Bekenstein en Hawking in de jaren zeventig lieten zien, hebben zwarte gaten een entropie die schaalt met het oppervlak van hun horizon. Voor thermodynamische systemen komt entropie overeen met het aantal microstaten dat correspondeert met een bepaalde macrostaat. Lange tijd was het niet duidelijk wat deze microstaten zouden kunnen zijn voor zwarte gaten. Om ze te begrijpen heb je een theorie nodig die kwantumgravitatie beschrijft. Een kandidaat hiervoor is snaartheorie die snaren in plaats van puntdeeltjes gebruikt om de fundamentele vrijheidsgraden te beschrijven. Ruimtetijd binnen de snaartheorie is tiendimensionaal, waarvan zes dimensies klein moeten zijn om onze wereld vierdimensionaal te laten lijken. Als deze theorie echt een kwantumgravitatietheorie is, dan moet het in ieder geval mogelijk zijn om de microstaten van zwarte gaten te tellen. In de jaren negentig werd aangetoond dat dit inderdaad mogelijk is. Zwarte gaten corresponderen in de snaartheorie met meerdimensionale objecten, branen, die opgerold zijn in de compacte dimensies. Deze branen kunnen op twee verschillende manieren worden beschreven. De eerste is met een oplossing van een supergravitatietheorie, een supersymmetrische uitbreiding van de algemene relativiteitstheorie. De tweede manier is door middel van de theorie die op de braan leeft en die de exitaties van met de braan verbonden open snaren beschrijft. Het is deze theorie die we kunnen gebruiken om microstaten te tellen. De specifieke zwarte gaten die wij bestuderen, hebben een hoger-dimensionale beschrijving als een zwarte snaar. De theorie op de braan kan dan worden gecompactificeerd naar de vrijheidsgraden van deze snaar. Dit resulteert in een tweedimensionale theorie die in de lage energie limiet een $2 \mathrm{~d}$ superconforme veldentheorie wordt. De entropie wordt vervolgens via de Cardy-formule uitgedrukt in karakteristieke data van deze theorie, namelijk in de centrale ladingen en niveaus. De Cardy-formule bevat ook correcties op de oppervlakteformule voor de entropie. Om deze entropie te reproduceren met behulp van de macroscopische supergravitatieoplossing, gebruiken we dat de geometrie dicht bij de waarnemingshorizon van een zwarte snaar een $\mathrm{AdS}_{3}$ factor bevat. De AdS/CFT-correspondentie vertelt ons vervolgens dat de centrale ladingen en niveaus corresponderen met de coëfficiënten van bepaalde Chern- 
Simons-termen in een effectieve $3 \mathrm{~d}$ actie. We kunnen dus de centrale ladingen en niveaus bepalen door de relevante supergravitatieactie in de geometrie dichtbij de waarnemingshorizon te reduceren naar een effectieve $3 \mathrm{~d}$ actie en de coëfficiënten van de relevante Chern-Simons-termen te bepalen. Om ook vrijheidsgraden buiten de waarnemingshorizon mee te nemen, blijkt dat deze reductie eigenlijk asymptotisch ver verwijderd van de waarnemingshorizon uitgevoerd moet worden.

In dit proefschrift hebben we de hierboven beschreven technieken toegepast op zwarte snaren in compactificaties van F-theorie op een elliptisch gevezelde Calabi-Yau drievariëteit of in de compactificatie van type IIB snaartheorie op K3 De zwarte snaar correspondeert met een D3-braan die op een tweedimensionaal oppervlak in de basis van de Calabi-Yau variëteit gewikkeld is of op een oppervlak in K3. De compactificaties van F-theorie resulteren in effectieve zesdimensionale $\mathcal{N}=(1,0)$ supergravitatietheorieën uitgedrukt in topologische data van de CalabiYau variëteit. Deze theorieën hebben een gravitatiemultiplet, tensormultipletten, hypermultipletten en vectormultipletten, maar voor de oplossingen die zwarte snaren beschrijven, kunnen we altijd the scalairen in the hypermultipletten constant nemen en de vectoren op nul zetten. De relevante zwarte snaren zijn daarom oplossingen van de $6 \mathrm{~d} \mathcal{N}=(1,0)$ supergravitatietheorie, gekoppeld aan tensormultipletten. Deze theorie is ook een truncatie van de $6 \mathrm{~d} \mathcal{N}=(2,0)$ supergravitatietheorie die de effectieve beschrijving is van type IIB supergravitatie gecompactificeerd op K3. In hoofdstuk 2 hebben we daarom supersymmetrische oplossingen bestudeerd van de $6 \mathrm{~d} \mathcal{N}=(1,0)$ supergravitatietheorie gekoppeld aan tensormultipletten. We hebben eerst een algemene lokale vorm afgeleid voor deze oplossingen, waarna we speciale gevallen hebben onderzocht waarvoor we de resulterende vergelijkingen expliciet konden oplossen. Op deze manier hebben we in het bijzonder uitdrukkingen afgeleid voor de zwarte snaar oplossingen die van belang waren in de hoofdstukken 3.5.

In hoofdstuk 3 hebben we zwarte snaren in F-theorie onderzocht waarbij de transversale dimensies bestaan uit een Taub-NUT ruimte. Deze ruimte is een cirkelvezeling over $\mathbb{R}^{3}$ en daarom correspondeert deze setup met vierdimensionale zwarte gaten. Verder beperkten we ons tot compactificaties van F-theorie op elliptisch gevezelde Calabi-Yau drievariëteiten waarbij de degeneraties van de vezels niet leiden tot singulariteiten van de hele vezeling. Dit correspondeert met zesdimensionale supergravitietheorieën die geen vectormultipletten bevatten Gebruikmakend van de zesdimensionale supergravitatietheorie hebben we de centrale ladingen en niveaus van de conforme veldentheorie gereproduceerd, die microscopisch al berekend waren via M-theorie 75]. We gebruikten dat de macroscopische ladingen niet gelijk zijn aan de microscopische ladingen, maar verschoven zijn 
met een constante in termen van de geometrie. Dit wordt veroorzaakt door de Green-Schwarz-term in de pseudoactie. Het reduceren asymptotisch ver weg van de waarnemingshorizon en de inclusie van één-lus Chern-Simons-termen komende van het uit-integreren van massieve Kaluza-Klein-deeltjes, zijn cruciale ingrediënten voor de niet-triviale matching.

In hoofdstuk 4 hebben we de macroscopische berekeningen van hoofdstuk 3 op twee manieren gegeneraliseerd. Ten eerste hebben we de transversale Taub-NUTruimte vervangen door een willekeurige asymptotisch lokaal Euclidische (ALE) of asymptotisch lokaal platte (ALP) ruimte. We hebben de corresponderende centrale ladingen en niveaus bepaald met de eerder beschreven technieken. Ten tweede hebben we ook Calabi-Yau vezelingen beschouwd, waarbij de degeneraties leiden tot singulariteiten van de hele vezeling. Dit resulteert in vectormultipletten en geladen hypermultipletten in de $6 \mathrm{~d} \mathcal{N}=(1,0)$ supergravitatietheorie. De vectormultipletten corresponderen met een ijksymmetrie in de $6 \mathrm{~d}$ bulktheorie. Deze bulksymmetrie leidt vervolgens tot flavoursymmetrieën in de theorie die leeft op de vrijheidsgraden van de snaar. Deze niet-abelse symmetrieën hebben ook niveaus die we bepaald hebben met behulp van de $6 \mathrm{~d}$ supergravitatietheorie. Net als voorheen corresponderen ze met de coëfficiënten van Chern-Simons-termen in de effectieve driedimensionale actie. We hebben verder laten zien dat de vectormultipletten en geladen hypermultipletten de waarden van de eerder berekende centrale ladingen en niveaus niet veranderen als deze uitgedrukt zijn in de microscopische ladingen en geometrische data.

Voor de setups van hoofdstuk 4 kunnen de macroscopische waarden voor de centrale ladingen en niveaus alleen worden vergeleken met het resultaat van een microscopische berekening wanneer de transversale ruimte gelijk is aan Taub-NUT of aan $\mathbb{R}^{4}$. Daarom hebben we in hoofdstuk 5 zwarte snaren met transversale ALE of ALP ruimtes bestudeerd in compactificaties van type IIB snaartheorie op K3. We berekenden opnieuw de centrale ladingen en niveaus met behulp van de $6 \mathrm{~d}$ supergravitatietheorie. Wanneer de transversale ruimte ALE of Taub-NUT is, is er een complementaire microscopische berekening. Net als in de F-theorie setup kan de microscopische berekening voor Taub-NUT als transversale ruimte gedaan worden via de duale M-theorie setup. Voor een transversale ALE ruimte beschouwden we eerst de theorie op een D3-braan met transversaal die specifieke ruimte. Dit is een 4 d ijktheorie met $\mathcal{N}=2$ supersymmetrie. Vervolgens compactificeerden we deze theorie op het riemann-oppervlak waarop de braan gewikkeld is. Om $\mathcal{N}=(0,4)$ supersymmetrie in de $2 \mathrm{~d}$ theorie te behouden, is een topologische twisting nodig. De centrale ladingen en niveaus van de conforme veldentheorie corresponderen met 
bepaalde anomaliecoëfficiënten die door 't Hooft-anomaliematching in de UV-theorie berekend kunnen worden. We vonden een perfecte match met de macroscopische berekeningen.

In hoofdstuk 6 zijn we een iets andere weg ingeslagen. We hebben de IR-limieten van compactificaties van de $4 \mathrm{~d} L^{a, b, c} \mathcal{N}=1$ ijktheorieën op een riemann-oppervlak bestudeerd met flux van globale symmetrieën. Ervan uitgaande dat ze in het IR met een $\mathcal{N}=(0,2)$ superconforme veldentheorie corresponderen, hebben we de centrale ladingen en R-ladingen van deze IR-theorie op drie verschillende manieren berekend. In de veldentheorie hebben we dit gedaan door gebruik te maken van de techniek van c-extremisatie. Met kennis van de UV-data is het dan mogelijk om de centrale lading en R-ladingen te berekenen dankzij 't Hooft-anomaliematching. De tweede manier is de recent gevonden geometrisch duale formulatie van c-extremisatie. Deze techniek heeft alleen topologische gegevens van de oplossing nodig waarbij de aanname is dat de supergravitatieoplossing ook daadwerkelijk bestaat. Ten slotte hebben we expliciete type IIB supergravitatie oplossingen geconstrueerd, die duaal zijn aan $2 \mathrm{~d} \mathcal{N}=(0,2)$ superconforme veldentheorieën. Deze oplossingen bevatten een $\mathrm{AdS}_{3}$ factor en kunnen beschouwd worden als de geometrie dichtbij de waarnemingshorizon van zwarte snaren in een ruimte die asymptotisch $\operatorname{AdS}_{5} \times L^{a, b, c}$ is. We hebben de oplossingen gebruikt om de centrale ladingen en R-ladingen van de duale veldentheorieën direct te berekenen. We vonden dat de veldtheoretische waarden exact overeenkomen met de geometrische waarden. Aangezien de centrale lading gerelateerd is aan de entropie dichtheid van de snaren, kan de matching van beide waarden gezien worden als het microscopisch tellen van de microstaten van de snaar. 


\section{Acknowledgements}

This thesis marks the end of four years of physics research and it has been a wonderful time. Many people have contributed to these years, each in their own and unique way.

First and foremost, I would like to thank my supervisor. Stefan, dank dat ik dit $\mathrm{PhD}$ traject kon doen onder jouw begeleiding, maar dat je me ook de vrijheid hebt gegeven om onafhankelijk van jou aan projecten te werken. Je deur was altijd open voor vragen (als ik maar laat genoeg kwam) ondanks je drukke posities, eerst als hoofd van het instituut en later als hoofd van het departement natuurkunde. Ik ben je hier zeer erkentelijk voor.

Also many thanks to Thomas, I almost thought of you as a second supervisor. Your detailed understanding of very abstract topics has always impressed me a lot. Collaborating with you was a pleasure! The conversations we have had about topics other than physics were also very enjoyable. I hope there will be a time you like traveling as much as I do.

Working actively together with other $\mathrm{PhD}$ students is much more fun than working on your own is what I figured out quickly. Kilian, we have collaborated on four projects over the past years and it was a pleasure working with you. It was great to have discussions with someone in which you did not have to be afraid of asking stupid questions. I have learned a lot from you and I am very happy I can defend this thesis with you being one of my paranymphs. The only thing I regret is that we never wrote this Swampland paper. Also keep suggesting 'shitty countries' to me, I'll gladly try to cross them off the list.

Chris, two years ago you came to Utrecht as a postdoc and over these years we have collaborated on three projects. I guess this kind of made up for you scooping me on my first $\mathrm{PhD}$ project. I have learned a lot from you over these years and I am very impressed by all the important research skills you already master. Also many thanks for your comments on a draft of the introduction of this thesis.

João, you asked me and Greg to collaborate with you on the highly interesting topic of exact entropy. The project came with its difficulties but I'm glad we made it work in the end. Many thanks to you and Greg.

The working environment is very important for someone's productivity. Therefore, 
I'd like to thank all members of the institute over the past four years. Adriana, Aron, Brice, Camilo, Chongchuo, Chris, Christian, Damian, Eric, Govert, Irene, Kilian, Koen, Lars, Markus, Matti, Miguel, Nava, Pierre, Phil, Raffaele, Rembert, Ronnie, Sander \& Sander, Sebastian, Stefan, Taka, Thomas, Tomislav, Umut, Watse, Wilke and many more, thanks for all the fun discussions over lunch and/or in the coffee room. Tomislav, I'm grateful for you being my master thesis supervisor and helping me to prepare the talk that eventually got me the $\mathrm{PhD}$ position. Self-proclaimed tori legends Koen \& Eric, thanks for discussions. Niccolò and Carmine, you gave our office the Italian touch.

Antonio, Beatrix, Carlos, Greg, John, Joren and Victor, you made the occasional holography meeting, DRSTP school or conference even more enjoyable. Carlos, it was a pleasure co-supervising you when you did your master thesis.

I would like to thank ESN Utrecht for keeping me busy when not doing physics. It is great to be a part of such a vibrant organization. Thanks to all the volunteers and internationals that have given me a social life in the past few years.

Dirk Jan, we hebben heel wat uren samen doorgebracht in de sportschool. Dank voor het aanhoren van al mijn klaagzangen en dank voor het zijn van mijn paranimf. Carlijn, alle theesessies met jou waren me een genoegen. Je bleef me vertellen om minder te gaan werken. Ik weet niet of ik uiteindelijk geluisterd heb.

My 'festival family' Anna, Elles, Melodie and Trang. I'm happy that we are still such good friends after that first year in the career committee. We have been to many festivals and gone on the occasional weekend trip together. I hope we will keep doing this for many years to come. Trang, thanks for being my buddy in the corona lockdown period during which this thesis was written. Melodie, dank voor je hulp bij het maken van de cover van dit proefschrift.

Edley, je was de eerste persoon die ik tegenkwam op de universiteit en ik ben blij dat we nog steeds vrienden zijn. Op nog vele momenten waarin je net zo lang doorpraat totdat ik me kapot schaam voor je!

Dank ook aan Chrystel, Peter en Zoë dat jullie het nog steeds uithouden met zo'n oude huisgenoot.

Last but definitely not least wil ik mijn familie bedanken. Jullie zijn er altijd voor me geweest en hebben me altijd mijn gang laten gaan. Deze doctorstitel is ook een beetje voor jullie. 


\section{About the author}

Huibert het Lam was born the 23rd of December 1991 in Tienhoven aan de Lek, the Netherlands. In 2010 he obtained his high school (VWO) diploma cum laude at the Gomarus scholengemeenschap in Gorinchem. In that same year, he started with a double bachelor program in mathematics and physics at Utrecht University which he completed cum laude in 2013. Besides the two bachelors he also completed an honours program. Part of this program was an extended bachelor research, which Huibert did in the computational astrophysics group at Leiden University

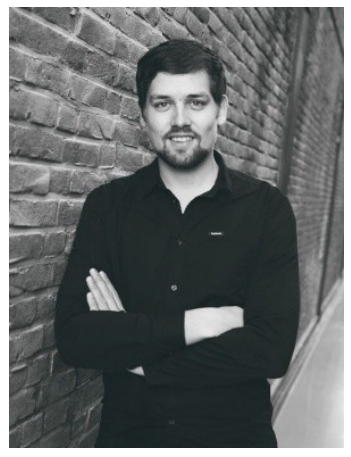
under supervision of Simon Portegies Zwart. He examined the merger times of stable triple black hole systems.

In 2013 Huibert started with a double master program in theoretical physics and mathematical sciences. The first semester of the academic year 2014-2015 he spent abroad at the Ruprecht-Karls-Universität in Heidelberg where he mainly followed graduate astrophysics courses. In 2016 he obtained both degrees cum laude, after writing a combined physics and mathematics master thesis on singularities in spacetimes with torsion under supervision of Tomislav Prokopec and Gil Cavalcanti.

In October 2016 he started as a PhD candidate at the Institute for Theoretical Physics in Utrecht. His position was special in the sense that everyone applying for it had to write a research proposal on a topic of their choosing. Huibert chose it to be centered around the entropy of black holes in F-theory and to have Stefan Vandoren as his supervisor. In four years, he performed research in collaboration with other researchers in Utrecht and elsewhere, part of which resulted in this thesis. During his time as a $\mathrm{PhD}$ student he also co-supervised two master students, taught problem classes, organized the Sinterklaas feast for the Physics department and organized PLaneTs which are talks by and for PhD students.

Besides physics, Huibert has been active in the Erasmus Student Network (ESN) which organizes activities for the international students in Utrecht. He also finds joy in exploring the world, in particular the less touristy areas. 



\section{Bibliography}

[1] H. het Lam and S. Vandoren, "BPS solutions of six-dimensional $(1,0)$ supergravity coupled to tensor multiplets," JHEP 06 (2018) 021, 1804.04681 .

[2] T. W. Grimm, H. het Lam, K. Mayer, and S. Vandoren, "Four-dimensional black hole entropy from F-theory," JHEP 01 (2019) 037, 1808.05228.

[3] C. Couzens, H. het Lam, K. Mayer, and S. Vandoren, "Black Holes and $(0,4)$ SCFTs from Type IIB on K3," JHEP 08 (2019) 043, 1904.05361.

[4] C. Couzens, H. het Lam, and K. Mayer, "Twisted $\mathcal{N}=1$ SCFTs and their $\mathrm{AdS}_{3}$ duals," JHEP 03 (2020) 032, 1912.07605.

[5] C. Couzens, H. het Lam, K. Mayer, and S. Vandoren, "Anomalies of $(0,4)$ SCFTs from F-theory," In preparation.

[6] H. het Lam and T. Prokopec, "Singularities in FLRW Spacetimes," Phys. Lett. B775 (2017) 311-314, 1606.01147.

[7] H. het Lam and T. Prokopec, "Singularities and Conjugate Points in FLRW Spacetimes," Gen. Rel. Grav. 49 (2017), no. 10, 133, 1704.03854

[8] J. Gomes, H. het Lam, and G. Mathys, "On the exact entropy of $\mathcal{N}=2$ black holes," 1912.00029

[9] R. Penrose, "Gravitational collapse and space-time singularities," Phys. Rev. Lett. 14 (1965) 57-59.

[10] S. Hawking and R. Penrose, "The Singularities of gravitational collapse and cosmology," Proc. Roy. Soc. Lond. A A314 (1970) 529-548.

[11] S. Hawking and G. Ellis, The Large Scale Structure of Space-Time. Cambridge Monographs on Mathematical Physics. Cambridge University Press, 2, 2011.

[12] B. Abbott et al., "Observation of Gravitational Waves from a Binary Black Hole Merger," Phys. Rev. Lett. 116 (2016), no. 6, 061102, 1602.03837.

[13] K. Akiyama et al., "First M87 Event Horizon Telescope Results. I. The Shadow of the Supermassive Black Hole," Astrophys. J. 875 (2019), no. 1, 
L1, 1906.11238 .

[14] J. D. Bekenstein, "Black holes and entropy," Phys. Rev. D 7 (1973) 2333-2346.

[15] J. M. Bardeen, B. Carter, and S. Hawking, "The Four laws of black hole mechanics," Commun. Math. Phys. 31 (1973) 161-170.

[16] J. D. Bekenstein, "Generalized second law of thermodynamics in black hole physics," Phys. Rev. D 9 (1974) 3292-3300.

[17] B. Carter, Black hole equilibrium states. Gordon and Breach, Science Publishers, Inc, United States, 1973.

[18] R. Ruffini and J. A. Wheeler, "Introducing the black hole," Phys. Today 24 (1971), no. 1, 30 .

[19] W. Israel, "Event Horizons in Static Vacuum Space-Times," Phys. Rev. 164 (Dec, 1967) 1776-1779.

[20] B. Carter, "Axisymmetric Black Hole Has Only Two Degrees of Freedom," Phys. Rev. Lett. 26 (1971) 331-333.

[21] D. Robinson, "Uniqueness of the Kerr black hole," Phys. Rev. Lett. 34 (1975) 905-906.

[22] P. Mazur, "Proof of uniqueness of the Kerr-Newman black hole solution," $J$. Phys. A 15 (1982) 3173-3180.

[23] S. Hawking, "Particle Creation by Black Holes," Commun. Math. Phys. 43 (1975) 199-220.

[24] R. M. Wald, "Black hole entropy is the Noether charge," Phys. Rev. D 48 (1993), no. 8, 3427-3431, gr-qc/9307038.

[25] V. Iyer and R. M. Wald, "Some properties of Noether charge and a proposal for dynamical black hole entropy," Phys. Rev. D 50 (1994) 846-864, gr-qc/9403028

[26] M. B. Green, J. Schwarz, and E. Witten, Superstring Theory. Vol. 1: Introduction. Cambridge Monographs on Mathematical Physics. 7, 1988.

[27] M. B. Green, J. Schwarz, and E. Witten, Superstring theory. Vol. 2: Loop amplitudes, anomalies and phenomenology. 7, 1988.

[28] J. Polchinski, String theory. Vol. 1: An introduction to the bosonic string. Cambridge Monographs on Mathematical Physics. Cambridge University Press, 12, 2007.

[29] J. Polchinski, String theory. Vol. 2: Superstring theory and beyond. 
Cambridge Monographs on Mathematical Physics. Cambridge University Press, 12, 2007.

[30] R. Blumenhagen, D. Lüst, and S. Theisen, Basic concepts of string theory. Theoretical and Mathematical Physics. Springer, Heidelberg, Germany, 2013.

[31] T. D. Brennan, F. Carta, and C. Vafa, "The String Landscape, the Swampland, and the Missing Corner," PoS TASI2017 (2017) 015, 1711.00864 .

[32] E. Palti, "The Swampland: Introduction and Review," Fortsch. Phys. 67 (2019), no. 6, 1900037, 1903.06239

[33] C. Vafa, "Evidence for F theory," Nucl. Phys. B469 (1996) 403-418, hep-th/9602022

[34] T. Weigand, "Lectures on F-theory compactifications and model building," Class. Quant. Grav. 27 (2010) 214004, 1009.3497.

[35] W. Taylor, "TASI Lectures on Supergravity and String Vacua in Various Dimensions," 1104.2051.

[36] T. Weigand, "F-theory," PoS TASI2017 (2018) 016, 1806.01854

[37] F. Bonetti and T. W. Grimm, "Six-dimensional $(1,0)$ effective action of F-theory via M-theory on Calabi-Yau threefolds," JHEP 05 (2012) 019, 1112.1082

[38] A. Strominger and C. Vafa, "Microscopic origin of the Bekenstein-Hawking entropy," Phys. Lett. B379 (1996) 99-104, hep-th/9601029

[39] C. G. Callan and J. M. Maldacena, "D-brane approach to black hole quantum mechanics," Nucl. Phys. B472 (1996) 591-610, hep-th/9602043

[40] A. W. Peet, "TASI lectures on black holes in string theory," in Strings, branes and gravity. Proceedings, Theoretical Advanced Study Institute, TASI'99, Boulder, USA, May 31-June 25, 1999, pp. 353-433. 8, 2000. hep-th/0008241

[41] J. M. Maldacena, Black holes in string theory. PhD thesis, Princeton U., 1996. hep-th/9607235

[42] J. L. Cardy, "Operator Content of Two-Dimensional Conformally Invariant Theories," Nucl. Phys. B 270 (1986) 186-204.

[43] J. Breckenridge, R. C. Myers, A. Peet, and C. Vafa, "D-branes and spinning black holes," Phys. Lett. B 391 (1997) 93-98, hep-th/9602065

[44] C. Vafa, "Black holes and Calabi-Yau threefolds," Adv. Theor. Math. Phys. 2 
(1998) 207-218, hep-th/9711067

[45] J. M. Maldacena, A. Strominger, and E. Witten, "Black hole entropy in M theory," JHEP 12 (1997) 002, hep-th/9711053.

[46] J. M. Maldacena and A. Strominger, "Statistical entropy of four-dimensional extremal black holes," Phys. Rev. Lett. 77 (1996) 428-429, hep-th/9603060

[47] C. V. Johnson, R. R. Khuri, and R. C. Myers, "Entropy of 4-D extremal black holes," Phys. Lett. B 378 (1996) 78-86, hep-th/9603061

[48] G. T. Horowitz and A. Strominger, "Counting states of near extremal black holes," Phys. Rev. Lett. 77 (1996) 2368-2371, hep-th/9602051.

[49] J. M. Maldacena, "The Large N limit of superconformal field theories and supergravity," Int. J. Theor. Phys. 38 (1999) 1113-1133, hep-th/9711200.

[50] S. Gubser, I. R. Klebanov, and A. M. Polyakov, "Gauge theory correlators from noncritical string theory," Phys. Lett. B 428 (1998) 105-114, hep-th/9802109

[51] E. Witten, "Anti-de Sitter space and holography," Adv. Theor. Math. Phys. 2 (1998) 253-291, hep-th/9802150

[52] J. P. Gauntlett, D. Martelli, J. Sparks, and D. Waldram, "Sasaki-Einstein metrics on $\mathrm{S}^{* *} 2 \times \mathrm{S}^{* *} 3, "$ Adv. Theor. Math. Phys. 8 (2004), no. 4, 711-734, hep-th/0403002

[53] M. Cvetic, H. Lu, D. N. Page, and C. N. Pope, "New Einstein-Sasaki spaces in five and higher dimensions," Phys. Rev. Lett. 95 (2005) 071101, hep-th/0504225

[54] H. Saida and J. Soda, "Statistical entropy of BTZ black hole in higher curvature gravity," Phys. Lett. B 471 (2000) 358-366, gr-qc/9909061

[55] P. Kraus and F. Larsen, "Microscopic black hole entropy in theories with higher derivatives," JHEP 09 (2005) 034, hep-th/0506176

[56] J. D. Brown and M. Henneaux, "Central Charges in the Canonical Realization of Asymptotic Symmetries: An Example from Three-Dimensional Gravity," Commun. Math. Phys. 104 (1986) 207-226.

[57] P. Kraus and F. Larsen, "Partition functions and elliptic genera from supergravity," JHEP 01 (2007) 002, hep-th/0607138.

[58] P. Kraus and F. Larsen, "Holographic gravitational anomalies," JHEP 01 (2006) 022, hep-th/0508218.

[59] J. Callan, Curtis G. and J. A. Harvey, "Anomalies and Fermion Zero Modes 
on Strings and Domain Walls," Nucl. Phys. B 250 (1985) 427-436.

[60] A. Dabholkar, J. Gomes, S. Murthy, and A. Sen, "Supersymmetric Index from Black Hole Entropy," JHEP 04 (2011) 034, 1009.3226.

[61] E. Witten, "Constraints on Supersymmetry Breaking," Nucl. Phys. B 202 (1982) 253.

[62] S. Cecotti, P. Fendley, K. A. Intriligator, and C. Vafa, "A New supersymmetric index," Nucl. Phys. B 386 (1992) 405-452, hep-th/9204102

[63] J. M. Maldacena, G. W. Moore, and A. Strominger, "Counting BPS black holes in toroidal Type II string theory," hep-th/9903163.

[64] A. Sen, "Arithmetic of Quantum Entropy Function," JHEP 08 (2009) 068, 0903.1477

[65] N. Banerjee, I. Mandal, and A. Sen, "Black Hole Hair Removal," JHEP 07 (2009) 091, 0901.0359

[66] D. P. Jatkar, A. Sen, and Y. K. Srivastava, "Black Hole Hair Removal: Non-linear Analysis," JHEP 02 (2010) 038, 0907.0593.

[67] A. Sen, "Quantum Entropy Function from $\operatorname{AdS}(2) / C F T(1)$ Correspondence," Int. J. Mod. Phys. A24 (2009) 4225-4244, 0809.3304

[68] A. Castro, D. Grumiller, F. Larsen, and R. McNees, "Holographic Description of AdS(2) Black Holes," JHEP 11 (2008) 052, 0809.4264

[69] F. Benini and N. Bobev, "Exact two-dimensional superconformal R-symmetry and c-extremization," Phys. Rev. Lett. 110 (2013), no. 6, $061601,1211.4030$

[70] F. Benini and N. Bobev, "Two-dimensional SCFTs from wrapped branes and c-extremization," JHEP 06 (2013) 005, 1302.4451.

[71] M. Bershadsky, A. Johansen, V. Sadov, and C. Vafa, "Topological reduction of $4 d$ SYM to $2 d$ sigma models," Nucl. Phys. B448 (1995) 166-186, hep-th/9501096

[72] M. Bershadsky, C. Vafa, and V. Sadov, "D-branes and topological field theories," Nucl. Phys. B463 (1996) 420-434, hep-th/9511222.

[73] J. M. Maldacena and C. Nunez, "Supergravity description of field theories on curved manifolds and a no go theorem," Int. J. Mod. Phys. A16 (2001) $822-855$, hep-th/0007018

[74] F. Benini, N. Bobev, and P. M. Crichigno, "Two-dimensional SCFTs from 
D3-branes," JHEP 07 (2016) 020, 1511.09462.

[75] I. Bena, D.-E. Diaconescu, and B. Florea, "Black string entropy and Fourier-Mukai transform," JHEP 04 (2007) 045, hep-th/0610068.

[76] B. Haghighat, S. Murthy, C. Vafa, and S. Vandoren, "F-Theory, Spinning Black Holes and Multi-string Branches," JHEP 01 (2016) 009, 1509.00455

[77] J. B. Gutowski, D. Martelli, and H. S. Reall, "All Supersymmetric solutions of minimal supergravity in six- dimensions," Class. Quant. Grav. 20 (2003) 5049-5078, hep-th/0306235

[78] M. Cariglia and O. A. P. Mac Conamhna, "The General form of supersymmetric solutions of $\mathrm{N}=(1,0) \mathrm{U}(1)$ and $\mathrm{SU}(2)$ gauged supergravities in six-dimensions," Class. Quant. Grav. 21 (2004) 3171-3196, hep-th/0402055

[79] D. Martelli and J. F. Morales, "Bubbling AdS(3)," JHEP 02 (2005) 048, hep-th/0412136

[80] I. Bena, S. Giusto, M. Shigemori, and N. P. Warner, "Supersymmetric Solutions in Six Dimensions: A Linear Structure," JHEP 03 (2012) 084, 1110.2781

[81] N. Bobev, B. E. Niehoff, and N. P. Warner, "New Supersymmetric Bubbles on $\mathrm{AdS}_{3} \mathrm{xS}^{3}$," JHEP 10 (2012) 013, 1204.1972

[82] B. E. Niehoff, O. Vasilakis, and N. P. Warner, "Multi-Superthreads and Supersheets," JHEP 04 (2013) 046, 1203.1348

[83] B. E. Niehoff and N. P. Warner, "Doubly-Fluctuating BPS Solutions in Six Dimensions," JHEP 10 (2013) 137, 1303.5449.

[84] S. Giusto, L. Martucci, M. Petrini, and R. Russo, "6D microstate geometries from 10D structures," Nucl. Phys. B876 (2013) 509-555, 1306.1745

[85] I. Bena, S. Giusto, R. Russo, M. Shigemori, and N. P. Warner, "Habemus Superstratum! A constructive proof of the existence of superstrata," JHEP 05 (2015) 110, 1503.01463

[86] P. de Lange, D. R. Mayerson, and B. Vercnocke, "Structure of Six-Dimensional Microstate Geometries," JHEP 09 (2015) 075, 1504.07987

[87] I. Bena, S. Giusto, E. J. Martinec, R. Russo, M. Shigemori, D. Turton, and N. P. Warner, "Asymptotically-flat supergravity solutions deep inside the black-hole regime," JHEP 02 (2018) 014, 1711.10474.

[88] P. A. Cano and T. Ortín, "The structure of all the supersymmetric solutions 
of ungauged $\mathcal{N}=(1,0), d=6$ supergravity," Class. Quant. Grav. 36 (2019), no. $12,125007,1804.04945$.

[89] R. Emparan and H. S. Reall, "Black Holes in Higher Dimensions," Living Rev. Rel. 11 (2008) 6, 0801.3471

[90] H. K. Kunduri and J. Lucietti, "Classification of near-horizon geometries of extremal black holes," Living Rev. Rel. 16 (2013) 8, 1306.2517.

[91] R. Emparan and H. S. Reall, "A Rotating black ring solution in five-dimensions," Phys. Rev. Lett. 88 (2002) 101101, hep-th/0110260.

[92] H. K. Kunduri and J. Lucietti, "Supersymmetric Black Holes with Lens-Space Topology," Phys. Rev. Lett. 113 (2014), no. 21, 211101, 1408.6083

[93] H. K. Kunduri and J. Lucietti, "Black lenses in string theory," Phys. Rev. D94 (2016), no. 6, 064007, 1605.01545

[94] S. Tomizawa and M. Nozawa, "Supersymmetric black lenses in five dimensions," Phys. Rev. D94 (2016), no. 4, 044037, 1606.06643

[95] M. Akyol and G. Papadopoulos, "Topology and geometry of 6-dimensional $(1,0)$ supergravity black hole horizons," Class. Quant. Grav. 29 (2012) $055002,1109.4254$

[96] G. W. Gibbons and C. M. Hull, "A Bogomolny Bound for General Relativity and Solitons in N=2 Supergravity," Phys. Lett. 109B (1982) 190-194.

[97] K. P. Tod, "All Metrics Admitting Supercovariantly Constant Spinors," Phys. Lett. 121B (1983) 241-244.

[98] K. P. Tod, "More on supercovariantly constant spinors," Class. Quant. Grav. 12 (1995) 1801-1820.

[99] F. Denef, "Supergravity flows and D-brane stability," JHEP 08 (2000) 050, hep-th/0005049

[100] P. Meessen and T. Ortin, "The Supersymmetric configurations of N=2, D=4 supergravity coupled to vector supermultiplets," Nucl. Phys. B749 (2006) 291-324, hep-th/0603099

[101] M. Huebscher, P. Meessen, and T. Ortin, "Supersymmetric solutions of N=2 $\mathrm{D}=4$ sugra: The Whole ungauged shebang," Nucl. Phys. B759 (2006) 228-248, hep-th/0606281

[102] J. P. Gauntlett, J. B. Gutowski, C. M. Hull, S. Pakis, and H. S. Reall, "All supersymmetric solutions of minimal supergravity in five- dimensions," Class. 
Quant. Grav. 20 (2003) 4587-4634, hep-th/0209114

[103] J. P. Gauntlett and J. B. Gutowski, "All supersymmetric solutions of minimal gauged supergravity in five-dimensions," Phys. Rev. D68 (2003) 105009, hep-th/0304064

[104] J. B. Gutowski and H. S. Reall, "General supersymmetric AdS(5) black holes," JHEP 04 (2004) 048, hep-th/0401129

[105] J. Bellorin, P. Meessen, and T. Ortin, "All the supersymmetric solutions of $\mathrm{N}=1, \mathrm{~d}=5$ ungauged supergravity," JHEP 01 (2007) 020, hep-th/0610196

[106] M. Akyol and G. Papadopoulos, "Spinorial geometry and Killing spinor equations of 6-D supergravity," Class. Quant. Grav. 28 (2011) 105001, 1010.2632

[107] G. W. Gibbons and S. W. Hawking, "Gravitational Multi - Instantons," Phys. Lett. 78B (1978) 430.

[108] P. M. Crichigno, F. Porri, and S. Vandoren, "Bound states of spinning black holes in five dimensions," JHEP 05 (2017) 101, 1603.09729

[109] L. J. Romans, "Selfduality for Interacting Fields: Covariant Field Equations for Six-dimensional Chiral Supergravities," Nucl. Phys. B276 (1986) 71.

[110] A. Sagnotti, "A Note on the Green-Schwarz mechanism in open string theories," Phys. Lett. B294 (1992) 196-203, hep-th/9210127

[111] S. Ferrara, F. Riccioni, and A. Sagnotti, "Tensor and vector multiplets in six-dimensional supergravity," Nucl. Phys. B519 (1998) 115-140, hep-th/9711059

[112] P. Pasti, D. P. Sorokin, and M. Tonin, "On Lorentz invariant actions for chiral p forms," Phys. Rev. D55 (1997) 6292-6298, hep-th/9611100

[113] G. Dall'Agata, K. Lechner, and M. Tonin, "Covariant actions for N=1, D = 6 supergravity theories with chiral bosons," Nucl. Phys. B512 (1998) 179-198, hep-th/9710127.

[114] J. Ford, S. Giusto, and A. Saxena, "A Class of BPS time-dependent 3-charge microstates from spectral flow," Nucl. Phys. B790 (2008) 258-280, hep-th/0612227

[115] O. Lunin, S. D. Mathur, and D. Turton, "Adding momentum to supersymmetric geometries," Nucl. Phys. B868 (2013) 383-415, 1208.1770

[116] M. Shigemori, "Perturbative 3-charge microstate geometries in six dimensions," JHEP 10 (2013) 169, 1307.3115. 
[117] M. Gunaydin, G. Sierra, and P. K. Townsend, "The Geometry of N=2 Maxwell-Einstein Supergravity and Jordan Algebras," Nucl. Phys. B242 (1984) 244-268.

[118] J. P. Gauntlett and J. B. Gutowski, "General concentric black rings," Phys. Rev. D71 (2005) 045002, hep-th/0408122

[119] F. Bonetti, T. W. Grimm, and S. Hohenegger, "One-loop Chern-Simons terms in five dimensions," JHEP 07 (2013) 043, 1302.2918

[120] G. W. Gibbons and P. J. Ruback, "The Hidden Symmetries of Multicenter Metrics," Commun. Math. Phys. 115 (1988) 267.

[121] I. Bena and N. P. Warner, "Bubbling supertubes and foaming black holes," Phys. Rev. D74 (2006) 066001, hep-th/0505166.

[122] I. Bena, N. Bobev, and N. P. Warner, "Spectral Flow, and the Spectrum of Multi-Center Solutions," Phys. Rev. D77 (2008) 125025, 0803.1203.

[123] I. Bena and N. P. Warner, "Black holes, black rings and their microstates," Lect. Notes Phys. 755 (2008) 1-92, hep-th/0701216.

[124] H. Elvang, R. Emparan, D. Mateos, and H. S. Reall, "A Supersymmetric black ring," Phys. Rev. Lett. 93 (2004) 211302, hep-th/0407065.

[125] H. Elvang, R. Emparan, D. Mateos, and H. S. Reall, "Supersymmetric black rings and three-charge supertubes," Phys. Rev. D71 (2005) 024033, hep-th/0408120

[126] G. T. Horowitz and D. Marolf, "Counting states of black strings with traveling waves," Phys. Rev. D55 (1997) 835-845, hep-th/9605224.

[127] G. T. Horowitz and D. Marolf, "Counting states of black strings with traveling waves. 2.," Phys. Rev. D55 (1997) 846-852, hep-th/9606113.

[128] N. Kaloper, R. C. Myers, and H. Roussel, "Wavy strings: Black or bright?," Phys. Rev. D55 (1997) 7625-7644, hep-th/9612248

[129] G. T. Horowitz and H.-s. Yang, "Black strings and classical hair," Phys. Rev. D55 (1997) 7618-7624, hep-th/9701077

[130] S. F. Ross, "Singularities in wavy strings," JHEP 08 (1998) 003, hep-th/9710158

[131] C. Couzens, C. Lawrie, D. Martelli, S. Schafer-Nameki, and J.-M. Wong, "F-theory and $\mathrm{AdS}_{3} / \mathrm{CFT}_{2}$," JHEP 08 (2017) 043, 1705.04679

[132] C. Couzens, D. Martelli, and S. Schafer-Nameki, "F-theory and $\mathrm{AdS}_{3} / \mathrm{CFT}_{2}$ (2, 0)," JHEP 06 (2018) 008, 1712.07631. 
[133] D. Gaiotto, A. Strominger, and X. Yin, "New connections between 4-D and 5-D black holes," JHEP 02 (2006) 024, hep-th/0503217

[134] K. Behrndt, G. Lopes Cardoso, and S. Mahapatra, "Exploring the relation between 4-D and 5-D BPS solutions," Nucl. Phys. B732 (2006) 200-223, hep-th/0506251

[135] S. Ferrara, R. Minasian, and A. Sagnotti, "Low-energy analysis of M and F theories on Calabi-Yau threefolds," Nucl. Phys. B474 (1996) 323-342, hep-th/9604097

[136] J. Hansen and P. Kraus, "Generating charge from diffeomorphisms," JHEP 12 (2006) 009, hep-th/0606230.

[137] C. Hull, E. Marcus, K. Stemerdink, and S. Vandoren, "Black holes in string theory with duality twists," 2003.11034

[138] H. Nishino and E. Sezgin, "New couplings of six-dimensional supergravity," Nucl. Phys. B505 (1997) 497-516, hep-th/9703075.

[139] D. R. Morrison and C. Vafa, "Compactifications of F theory on Calabi-Yau threefolds. 1," Nucl. Phys. B473 (1996) 74-92, hep-th/9602114.

[140] D. R. Morrison and C. Vafa, "Compactifications of F theory on Calabi-Yau threefolds. 2.," Nucl. Phys. B476 (1996) 437-469, hep-th/9603161.

[141] V. Sadov, "Generalized Green-Schwarz mechanism in F theory," Phys. Lett. B388 (1996) 45-50, hep-th/9606008

[142] I. Antoniadis, S. Ferrara, R. Minasian, and K. S. Narain, "R**4 couplings in $\mathrm{M}$ and type II theories on Calabi-Yau spaces," Nucl. Phys. B507 (1997) 571-588, hep-th/9707013.

[143] T. W. Grimm, K. Mayer, and M. Weissenbacher, "Higher derivatives in Type II and M-theory on Calabi-Yau threefolds," JHEP 02 (2018) 127, 1702.08404

[144] T. W. Grimm and W. Taylor, "Structure in 6D and 4D N=1 supergravity theories from F-theory," JHEP 10 (2012) 105, 1204.3092

[145] S. B. Giddings, S. Kachru, and J. Polchinski, "Hierarchies from fluxes in string compactifications," Phys. Rev. D66 (2002) 106006, hep-th/0105097

[146] A. Castro, J. L. Davis, P. Kraus, and F. Larsen, "Precision Entropy of Spinning Black Holes," JHEP 09 (2007) 003, 0705.1847.

[147] A. Castro, J. L. Davis, P. Kraus, and F. Larsen, "5D Black Holes and Strings with Higher Derivatives," JHEP 06 (2007) 007, hep-th/0703087. 
[148] P. A. Cano, S. Chimento, P. Meessen, T. Ortín, P. F. Ramírez, and

A. Ruipérez, "Beyond the near-horizon limit: Stringy corrections to Heterotic Black Holes," JHEP 02 (2019) 192, 1808.03651.

[149] J. R. David and A. Sen, "CHL Dyons and Statistical Entropy Function from D1-D5 System," JHEP 11 (2006) 072, hep-th/0605210

[150] P. K. Townsend, K. Pilch, and P. van Nieuwenhuizen, "Selfduality in Odd Dimensions," Phys. Lett. 136B (1984) 38.

[151] F. Bonetti, T. W. Grimm, and S. Hohenegger, "A Kaluza-Klein inspired action for chiral p-forms and their anomalies," Phys. Lett. B720 (2013) 424-427, 1206.1600

[152] S. Deger, A. Kaya, E. Sezgin, and P. Sundell, "Spectrum of D =6, N=4b supergravity on AdS in three-dimensions x S**3," Nucl. Phys. B536 (1998) 110-140, hep-th/9804166.

[153] J. de Boer, "Six-dimensional supergravity on $\mathrm{S}^{* *} 3 \times \operatorname{AdS}(3)$ and 2-D conformal field theory," Nucl. Phys. B548 (1999) 139-166, hep-th/9806104.

[154] M. F. Atiyah, V. K. Patodi, and I. M. Singer, "Spectral asymmetry and Riemannian Geometry 1," Math. Proc. Cambridge Phil. Soc. 77 (1975) 43.

[155] M. F. Atiyah, V. K. Patodi, and I. M. Singer, "Spectral asymmetry and Riemannian geometry 2," Math. Proc. Cambridge Phil. Soc. 78 (1976) 405.

[156] M. F. Atiyah, V. K. Patodi, and I. M. Singer, "Spectral asymmetry and Riemannian geometry. III," Math. Proc. Cambridge Phil. Soc. 79 (1976) 71-99.

[157] G. Ishiki, Y. Takayama, and A. Tsuchiya, "N=4 SYM on R x S**3 and theories with 16 supercharges," JHEP 10 (2006) 007, hep-th/0605163.

[158] L. Alvarez-Gaume, S. Della Pietra, and G. W. Moore, "Anomalies and Odd Dimensions," Annals Phys. 163 (1985) 288.

[159] L. Alvarez-Gaume and P. H. Ginsparg, "The Structure of Gauge and Gravitational Anomalies," Annals Phys. 161 (1985) 423.

[160] T. Eguchi, P. B. Gilkey, and A. J. Hanson, "Gravitation, Gauge Theories and Differential Geometry," Phys. Rept. 66 (1980) 213.

[161] T. W. Grimm, E. Palti, and I. Valenzuela, "Infinite Distances in Field Space and Massless Towers of States," JHEP 08 (2018) 143, 1802.08264

[162] P. Corvilain, T. W. Grimm, and D. Regalado, "Chiral anomalies on a circle and their cancellation in F-theory," JHEP 04 (2018) 020, 1710.07626 
[163] N. Arkani-Hamed, S. Dimopoulos, and S. Kachru, "Predictive landscapes and new physics at a TeV," hep-th/0501082

[164] G. Dvali, "Black Holes and Large N Species Solution to the Hierarchy Problem," Fortsch. Phys. 58 (2010) 528-536, 0706.2050

[165] L. Martucci, "Topological duality twist and brane instantons in F-theory," JHEP 06 (2014) 180, 1403.2530

[166] C. Lawrie, S. Schafer-Nameki, and T. Weigand, "Chiral 2d theories from N = 4 SYM with varying coupling," JHEP 04 (2017) 111, 1612.05640.

[167] M. J. Duff, B. E. W. Nilsson, and C. N. Pope, "Kaluza-Klein Supergravity," Phys. Rept. 130 (1986) 1-142.

[168] T. Eguchi and A. J. Hanson, "Asymptotically flat self-dual solutions to euclidean gravity," Physics Letters B 74 (1978), no. 3, 249 - 251.

[169] P. B. Kronheimer, "The construction of ALE spaces as hyper-Kähler quotients," J. Diff. Geom. 29 (1989), no. 3, 665-683.

[170] S. A. Cherkis and A. Kapustin, "Singular monopoles and gravitational instantons," Commun. Math. Phys. 203 (1999) 713-728, hep-th/9803160

[171] S. W. Hawking, "Gravitational Instantons," Phys. Lett. A60 (1977) 81.

[172] S. A. Cherkis and N. J. Hitchin, "Gravitational instantons of type $\mathrm{D}(\mathrm{k})$," Commun. Math. Phys. 260 (2005) 299-317, hep-th/0310084

[173] A. S. Dancer, "Nahm's equations and hyperKahler geometry," Commun. Math. Phys. 158 (1993) 545-568.

[174] A. Sen, "A Note on enhanced gauge symmetries in M and string theory," JHEP 09 (1997) 001, hep-th/9707123

[175] G. W. Gibbons, C. N. Pope, and H. Romer, "Index Theorem Boundary Terms for Gravitational Instantons," Nucl. Phys. B157 (1979) 377-386.

[176] S. A. Cherkis, "Instantons on the Taub-NUT Space," Adv. Theor. Math. Phys. 14 (2010), no. 2, 609-642, 0902.4724

[177] E. Witten, "Branes, Instantons, And Taub-NUT Spaces," JHEP 06 (2009) 067, 0902.0948

[178] S. A. Cherkis, A. Larrain-Hubach, and M. Stern, "Instantons on multi-Taub-NUT Spaces I: Asymptotic Form and Index Theorem," 1608.00018

[179] H.-C. Kim, S. Kim, and J. Park, "6d strings from new chiral gauge theories," 1608.03919 . 
[180] H. Shimizu and Y. Tachikawa, "Anomaly of strings of $6 \mathrm{~d} \mathcal{N}=(1,0)$ theories," JHEP 11 (2016) 165, 1608.05894.

[181] H. Hayashi, P. Jefferson, H.-C. Kim, K. Ohmori, and C. Vafa, "SCFTs, Holography, and Topological Strings," 1905.00116

[182] B. Assel and S. Schafer-Nameki, "Six-dimensional origin of $\mathcal{N}=4$ SYM with duality defects," JHEP 12 (2016) 058, 1610.03663

[183] M. R. Douglas and G. W. Moore, "D-branes, quivers, and ALE instantons," hep-th/9603167

[184] C. V. Johnson and R. C. Myers, "Aspects of type IIB theory on ALE spaces," Phys. Rev. D55 (1997) 6382-6393, hep-th/9610140.

[185] F. Riccioni, "Tensor multiplets in six-dimensional $(2,0)$ supergravity," Phys. Lett. B422 (1998) 126-134, hep-th/9712176.

[186] P. K. Townsend, "A New Anomaly Free Chiral Supergravity Theory From Compactification on K3," Phys. Lett. 139B (1984) 283-287.

[187] N. Kim, "AdS 3 solutions of IIB supergravity from D3-branes," JHEP 01 (2006) 094, hep-th/0511029.

[188] P. Putrov, J. Song, and W. Yan, “(0,4) dualities," JHEP 03 (2016) 185, 1505.07110.

[189] N. Lambert, "The M5-brane on K3×T ${ }^{2}$," JHEP 02 (2008) 060, 0712.3166

[190] M. Cvetic and C. M. Hull, "Wrapped branes and supersymmetry," Nucl. Phys. B519 (1998) 141-158, hep-th/9709033.

[191] J. P. Gauntlett and O. A. P. Mac Conamhna, "AdS spacetimes from wrapped D3-branes," Class. Quant. Grav. 24 (2007) 6267-6286, 0707.3105.

[192] A. Kapustin, "Holomorphic reduction of $\mathrm{N}=2$ gauge theories, Wilson-'t Hooft operators, and S-duality," hep-th/0612119

[193] A. Amariti, L. Cassia, and S. Penati, "Surveying 4d SCFTs twisted on Riemann surfaces," JHEP 06 (2017) 056, 1703.08201

[194] A. Dabholkar, J. Gomes, and S. Murthy, "Counting all dyons in N =4 string theory," JHEP 05 (2011) 059, 0803.2692.

[195] A. Arabi Ardehali, F. Larsen, J. T. Liu, and P. Szepietowski, "Quantum corrections to central charges and supersymmetric Casimir energy in $\mathrm{AdS}_{3} / \mathrm{CFT}_{2}$," JHEP 07 (2019) 071, 1811.12367.

[196] M. Beccaria, G. Macorini, and A. A. Tseytlin, "Supergravity one-loop corrections on $\mathrm{AdS}_{7}$ and $\mathrm{AdS}_{3}$, higher spins and AdS/CFT," Nucl. Phys. 
B892 (2015) 211-238, 1412.0489.

[197] G. Lopes Cardoso, B. de Wit, and T. Mohaupt, "Macroscopic entropy formulae and nonholomorphic corrections for supersymmetric black holes," Nucl. Phys. B567 (2000) 87-110, hep-th/9906094

[198] A. Hanany and B. Kol, "On orientifolds, discrete torsion, branes and M theory," JHEP 06 (2000) 013, hep-th/0003025.

[199] D. Martelli, J. Sparks, and S.-T. Yau, "The Geometric dual of a-maximisation for Toric Sasaki-Einstein manifolds," Commun. Math. Phys. 268 (2006) 39-65, hep-th/0503183.

[200] D. Martelli, J. Sparks, and S.-T. Yau, "Sasaki-Einstein manifolds and volume minimisation," Commun. Math. Phys. 280 (2008) 611-673, hep-th/0603021

[201] A. Amariti, L. Cassia, and S. Penati, "c-extremization from toric geometry," Nucl. Phys. B929 (2018) 137-170, 1706.07752

[202] A. Donos, J. P. Gauntlett, and N. Kim, "AdS Solutions Through Transgression," JHEP 09 (2008) 021, 0807.4375

[203] J. P. Gauntlett, O. A. P. Mac Conamhna, T. Mateos, and D. Waldram, "Supersymmetric AdS(3) solutions of type IIB supergravity," Phys. Rev. Lett. 97 (2006) 171601, hep-th/0606221

[204] C. Couzens, J. P. Gauntlett, D. Martelli, and J. Sparks, "A geometric dual of c-extremization," JHEP 01 (2019) 212, 1810.11026

[205] J. P. Gauntlett, D. Martelli, and J. Sparks, "Toric geometry and the dual of c-extremization," JHEP 01 (2019) 204, 1812.05597

[206] J. P. Gauntlett, D. Martelli, and J. Sparks, "Fibred GK geometry and supersymmetric AdS solutions," JHEP 11 (2019) 176, 1910.08078

[207] F. Benini, K. Hristov, and A. Zaffaroni, "Black hole microstates in $\mathrm{AdS}_{4}$ from supersymmetric localization," JHEP 05 (2016) 054, 1511.04085.

[208] S. M. Hosseini and A. Zaffaroni, "Large $N$ matrix models for $3 \mathrm{~d} \mathcal{N}=2$ theories: twisted index, free energy and black holes," JHEP 08 (2016) 064, 1604.03122 .

[209] J. P. Gauntlett, D. Martelli, and J. Sparks, "Toric geometry and the dual of I-extremization," JHEP 06 (2019) 140, 1904.04282

[210] S. M. Hosseini and A. Zaffaroni, "Geometry of $\mathcal{I}$-extremization and black holes microstates," JHEP 07 (2019) 174, 1904.04269 
[211] H. Kim and N. Kim, "Black holes with baryonic charge and $\mathcal{I}$-extremization," JHEP 11 (2019) 050, 1904.05344

[212] S. M. Hosseini and A. Zaffaroni, "Proving the equivalence of $c$-extremization and its gravitational dual for all toric quivers," JHEP 03 (2019) 108, 1901.05977,

[213] P. Karndumri and E. O Colgáin, "Supergravity dual of $c$-extremization," Phys. Rev. D87 (2013), no. 10, 101902, 1302.6532

[214] P. Karndumri and E. O. Colgáin, "3D Supergravity from wrapped D3-branes," JHEP 10 (2013) 094, 1307.2086.

[215] S. Benvenuti and M. Kruczenski, "From Sasaki-Einstein spaces to quivers via BPS geodesics: L**p,q|r," JHEP 04 (2006) 033, hep-th/0505206

[216] S. Franco, A. Hanany, D. Martelli, J. Sparks, D. Vegh, and B. Wecht, "Gauge theories from toric geometry and brane tilings," JHEP 01 (2006) 128, hep-th/0505211

[217] A. Butti, D. Forcella, and A. Zaffaroni, "The Dual superconformal theory for L**pqr manifolds," JHEP 09 (2005) 018, hep-th/0505220

[218] S. Benvenuti, S. Franco, A. Hanany, D. Martelli, and J. Sparks, "An Infinite family of superconformal quiver gauge theories with Sasaki-Einstein duals," JHEP 06 (2005) 064, hep-th/0411264

[219] D. Martelli and J. Sparks, "Toric Sasaki-Einstein metrics on $\mathrm{S}^{* *} 2 \times \mathrm{S}^{* *} 3$," Phys. Lett. B621 (2005) 208-212, hep-th/0505027.

[220] V. Apostolov, D. M. Calderbank, and P. Gauduchon, "The geometry of weakly selfdual Kahler surfaces," math/0104233.

[221] J. P. Gauntlett and N. Kim, "Geometries with Killing Spinors and Supersymmetric AdS Solutions," Commun. Math. Phys. 284 (2008) 897-918, 0710.2590

[222] J. P. Gauntlett, N. Kim, and D. Waldram, "Supersymmetric $\mathrm{AdS}_{3}, \mathrm{AdS}_{2}$ and Bubble Solutions," JHEP 04 (2007) 005, hep-th/0612253

[223] J. P. Gauntlett, O. A. P. Mac Conamhna, T. Mateos, and D. Waldram, "New supersymmetric $\mathrm{AdS}_{3}$ solutions," Phys. Rev. D74 (2006) 106007, hep-th/0608055

[224] N. Kim, "The Backreacted Kähler Geometry of Wrapped Branes," Phys. Rev. D86 (2012) 067901, 1206.1536.

[225] L. Eberhardt, "Supersymmetric $\mathrm{AdS}_{3}$ supergravity backgrounds and 
holography," JHEP 02 (2018) 087, 1710.09826

[226] N. T. Macpherson, "Type II solutions on $\mathrm{AdS}_{3} \times \mathrm{S}^{3} \times \mathrm{S}^{3}$ with large superconformal symmetry," JHEP 05 (2019) 089, 1812.10172

[227] A. Passias and D. Prins, "On $\mathcal{N}=1 \mathrm{AdS}_{3}$ solutions of Type IIB," 1910.06326.

[228] C. Couzens, " $\mathcal{N}=(0,2) \mathrm{AdS}_{3}$ Solutions of Type IIB and F-theory with Generic Fluxes," 1911.04439

[229] Y. Bea, J. D. Edelstein, G. Itsios, K. S. Kooner, C. Nunez, D. Schofield, and J. A. Sierra-Garcia, "Compactifications of the Klebanov-Witten CFT and new $\mathrm{AdS}_{3}$ backgrounds," JHEP 05 (2015) 062, 1503.07527

[230] Y. Lozano, N. T. Macpherson, J. Montero, and E. O. Colgáin, "New $\mathrm{AdS}_{3} \times S^{2}$ T-duals with $\mathcal{N}=(0,4)$ supersymmetry," JHEP 08 (2015) 121 , 1507.02659 .

[231] G. Dibitetto, G. Lo Monaco, A. Passias, N. Petri, and A. Tomasiello, "AdS 3 Solutions with Exceptional Supersymmetry," Fortsch. Phys. 66 (2018), no. $10,1800060,1807.06602$

[232] Y. Lozano, N. T. Macpherson, C. Nunez, and A. Ramirez, "AdS 3 solutions in Massive IIA with small $\mathcal{N}=(4,0)$ supersymmetry," JHEP 01 (2020) 129, 1908.09851 .

[233] Y. Lozano, N. T. Macpherson, C. Nunez, and A. Ramirez, "1/4 BPS solutions and the $\mathrm{AdS}_{3} / \mathrm{CFT}_{2}$ correspondence," Phys. Rev. D 101 (2020), no. 2, 026014, 1909.09636

[234] Y. Lozano, N. T. Macpherson, C. Nunez, and A. Ramirez, "Two dimensional $\mathcal{N}=(0,4)$ quivers dual to $\mathrm{AdS}_{3}$ solutions in massive IIA," JHEP 01 (2020) $140,1909.10510$.

[235] Y. Lozano, N. T. Macpherson, C. Nunez, and A. Ramirez, "AdS ${ }_{3}$ solutions in massive IIA, defect CFTs and T-duality," JHEP 12 (2019) 013, 1909.11669

[236] K. A. Intriligator and B. Wecht, "The Exact superconformal R symmetry maximizes a," Nucl. Phys. B667 (2003) 183-200, hep-th/0304128

[237] M. Abreu, "Kähler geometry of toric manifolds in symplectic coordinates," math/0004122

[238] N. Bobev and P. M. Crichigno, "Universal RG Flows Across Dimensions and Holography," JHEP 12 (2017) 065, 1708.05052. 